GABRIEL PEDRO RAMOS MACIEL

MÉTODOS PARA REDUÇÃO DE GRAUS DE LIBERDADE EM SISTEMAS DINÂMICOS LINEARES 
GABRIEL PEDRO RAMOS MACIEL

\section{MÉTODOS PARA REDUÇÃO DE GRAUS DE LIBERDADE EM SISTEMAS DINÂMICOS LINEARES}

Dissertação apresentada à Escola Politécnica da Universidade de São Paulo para obtenção do título de mestre em Ciências

São Paulo 
GABRIEL PEDRO RAMOS MACIEL

\section{MÉTODOS PARA REDUÇÃO DE GRAUS DE LIBERDADE EM SISTEMAS DINÂMICOS LINEARES}

Dissertação apresentada à Escola Politécnica da Universidade de São Paulo para obtenção do título de mestre em Ciências

Área de concentração:

Engenharia de Controle e Automação Mecânica

Orientador:

Prof. Dr. Roberto Spinola Barbosa

São Paulo 


\section{Catalogação-na-publicação}

Maciel, Gabriel Pedro Ramos

Métodos para redução de graus de liberdade em sistemas dinâmicos lineares / Maciel, G. P. R. -- São Paulo, 2015.

$178 \mathrm{p}$.

Dissertação (Mestrado) - Escola Politécnica da Universidade de São Paulo. Departamento de Engenharia Mecânica.

1. sistemas dinâmicos 2. sistemas lineares 3. teoria de sistemas e controle 4. controlabilidade 5. observabilidade I. Universidade de São Paulo. Escola Politécnica. Departamento de Engenharia Mecânica II. t. 


\section{AGRADECIMENTOS}

Ao meu orientador Prof. Dr. Roberto Spinola Barbosa, por toda a ajuda na construção deste trabalho, obrigado pelas lições, confiança, paciência e dedicação nesta trajetória acadêmica.

Aos Professores da banca de qualificação Prof. Dr. Raul Gonzales Lima e Prof. Dr. Flávio Celso Trigo, pelos conselhos e sugestões para este trabalho.

À minha familia, principalmente à minha mãe Altair e avó Lioneza, sem vocês não teria chegado neste ponto. 
Peço-lhe que tente ter amor pelas próprias perguntas, como quartos fechados e como livros escritos em uma língua estrangeira. Não investigue agora as respostas que não lhe podem ser dadas, porque não poderá vivê-las. E é disto que se trata, de viver tudo. Viva agora as perguntas. Talvez passe gradativamente, em um belo dia, sem perceber, a viver as respostas (Rainer Maria Rilke, 1903) 


\section{RESUMO}

O objetivo deste estudo é apresentar uma revisão sobre técnicas de redução da ordem de modelos dinâmicos lineares e invariantes no tempo. Com a implementação de tais técnicas, o autor mostra que é possível reproduzir as principais características da resposta de um modelo de alta ordem através de um modelo de ordem reduzida com menor número de graus de liberdade.

Uma metodologia para redução da ordem de modelos de sistemas dinâmicos foi apresentada. Os processos envolvidos nesta metodologia foram descritos, os quais são: técnicas para realizar projeções do sistema em diferentes bases, selecionar os graus de liberdade que são bons candidatos a eliminação, eliminar graus de liberdade do modelo completo e implementar correções na resposta do modelo reduzido.

Foram apresentadas maneiras de quantificar as similitudes entre as respostas dos modelos completo e reduzido através de métricas de representatividade.

Para implementar e estudar as técnicas de redução apresentadas, o autor elaborou dois modelos para estudo de caso: um modelo para estudo da dinâmica vertical de um veículo de passeio e outro modelo para estudo da dinâmica longitudinal de um trem.

Diferentes técnicas de redução foram implementadas a partir dos dois modelos para estudo de caso e os resultados foram comparados através das métricas de representatividade. 0 critério proposto pelo autor para quantificar desempenho de um modelo reduzido foi utilizado para determinar de maneira objetiva o modelo reduzido mais adequado para cada aplicação.

Como contribuição neste trabalho, o autor propôs uma definição de desempenho de um modelo reduzido e um método para quantificar o mesmo, além de duas novas métricas para mensurar a capacidade do modelo reduzido em reproduzir os máximos sobre-sinais e tempos de acomodação do modelo completo.

Palavras-chave: redução da ordem de modelos. sistemas dinâmicos. sistemas lineares. teoria de sistemas e controle. representatividade de modelos reduzidos. 


\begin{abstract}
The objective of this work is to present a revision about model order reduction techniques applied to linear, time invariant dynamic systems. With the implementation of these techniques, the author shows that it is possible to reproduce the main characteristics of the response of a high order dynamic system using a reduced order model with fewer degrees of freedom.

A model order reduction methodology was presented. The processes which are involved in this methodology were described, which are: techniques for projection onto different basis, selection of the most suitable degrees of freedom to be reduced, elimination of degrees of freedom from the high order model, implementation of corrections at the reduced model response.

The author showed ways to quantify the similarities between the responses of the complete and reduced models using representativeness metrics.

In order to implement and study the presented model order reduction techniques, the author developed two case study models: one model to study the vertical dynamics of a passenger car and another model to study the longitudinal dynamics of a train.

Different model order reduction techniques were implemented and its results were compared using representativeness metrics and the performance of the reduced models. The criteria proposed by the author to quantify the performance of a reduced order model was used to objectively determine the most suitable reduced order model for each application.

The author proposed, as contribution at this work, a definition of the reduced order model performance, a method to quantify its performance and two new metrics to measure the capacity of the reduced model to reproduce the overshoots and settling times of the complete model.
\end{abstract}

Keywords: model order reduction. dynamical systems. linear systems. systems and control theory. representativeness of reduced order models 


\section{LISTA DE ILUSTRAÇÕES}

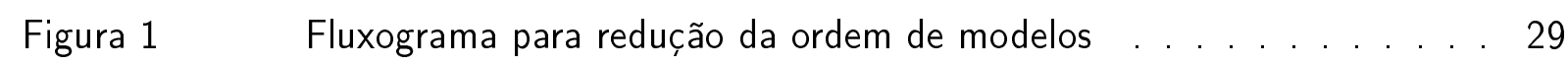

Figura 2 Exemplo de modo assíncrono. . . . . . . . . . . . . . . . . 42

Figura 3 Exemplo da participação das funções de transferência dos modos na

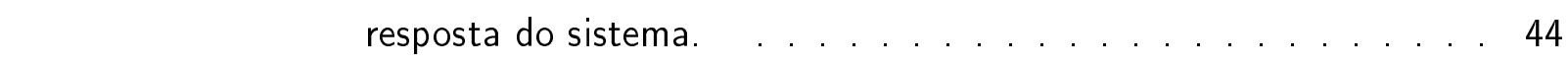

Figura $4 \quad$ Representação em $\mathbb{R}^{2}$ do operador $A$ diagonalizado pela DVS . . . . . 46

Figura $5 \quad$ Comportamento indesejado dos vetores que formam a base de Krylov. 50

Figura $6 \quad$ Ortogonalização de um novo vetor da base de Krylov pelo método de

Arnoldi. . . . . . . . . . . . . . . . . 52

Figura $7 \quad$ Autovalor de um sistema dinâmico no plano complexo. . . . . . . . . . 56

Figura $8 \quad$ Critério de seleção baseado na frequência natural dos autovalores. . . . 57

Figura $9 \quad$ Critério de seleção baseado na parte real dos autovalores . . . . . . . 58

Figura $10 \quad$ Exemplo de normas Hankel aproximadas pelos blocos da base modal. 59

Figura $11 \quad$ Exemplo de correção para resposta estática. $\ldots$. . . . . . . . . . . . 78

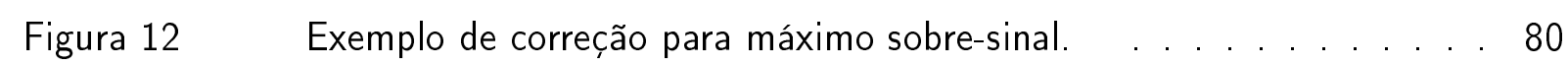

Figura 13 Exemplo gráfico do sobre-sinal. . . . . . . . . . . . . . . . . . . . . . 84

Figura $14 \quad$ Exemplo gráfico do tempo de acomodação. . . . . . . . . . . . . . . 85

Figura $15 \quad$ Exemplo gráfico da norma $\|G\|_{\mathcal{H}_{\infty}}$ de um sistema SISO. . . . . . . . 87

Figura 16 Modelo de veículo de passeio . . . . . . . . . . . . . . . . . . . . . . . 94

Figura 17 Acoplamento entre veículos ferroviários. Fonte: (BARBOSA, 1993) . 98

Figura 18 Modelo de trem. . . . . . . . . . . . . . . . . . . . . . . . . 98

Figura $19 \quad$ Forças externas do modelo, representando tração das locomotivas. . . 99

Figura 20 Diagrama de polos e zeros do sistema . . . . . . . . . . . . . . 112

Figura $21 \quad$ Formas modais do primeiro (esquerda) e segundo (direita) modos de vibrar do SC . . . . . . . . . . . . . . . . . . . . . . . . . . 113

Figura $22 \quad$ Formas modais do terceiro (esquerda) modo de vibrar do SC . . . . . 114

Figura $23 \quad$ Formas modais do quarto (esquerda) e quinto (direita) modos de vibrar do SC . . . . . . . . . . . . . . . . . 114

Figura $24 \quad$ Formas modais do sexto (esquerda) e sétimo (direita) modos de vibrar do SC . . . . . . . . . . . . . . . . . . . . 114

Figura $25 \quad$ Parâmetro de seleção $P_{\omega_{n}}$ baseado no critério CSF . . . . . . . . . 116 
Figura $26 \quad$ Representação gráfica dos modos mais e menos dominantes segundo critério CSF . . . . . . . . . . . . . . . . . . 116

Figura 27 Parâmetro de seleção $P_{a f}$ baseado no critério CSFA . . . . . . . . . . 117

Figura $28 \quad$ Representação gráfica dos modos mais e menos dominantes segundo critério CSFA . . . . . . . . . . . . . . . . . . . . . . 118

Figura 29 Comparação entre as normas Hankel do sistema completo e dos modos de vibrar . . . . . . . . . . . . . . . . . . . . . . . . . . . . . . . . 119

Figura $30 \quad$ Parâmetro de seleção $P_{\gamma m}$ baseado no critério CSCO . . . . . . . . . 119

Figura $31 \quad$ Máximo valor singular da função de transferência dos modos de vibrar do sistema completo . . . . . . . . . . . . . . . . . . . . . . 120

Figura $32 \quad$ Parâmetro de seleção $P_{s}$ baseado no critério CSFT . . . . . . . . . . 121

Figura $33 \quad$ Parâmetro de seleção $P_{\sigma}$ baseado no critério CSVS . . . . . . . . . 122

Figura $34 \quad$ Parâmetro de seleção $P_{\gamma}$ baseado no critério CSNH . . . . . . . . . . 123

\begin{tabular}{llll}
\hline Figura 35 & Ângulo de rolagem para entrada degrau de $100 \mathrm{~mm}$
\end{tabular} . . . . . . . . . . . 128

\begin{tabular}{llll}
\hline Figura 36 & Erro do ângulo de rolagem para entrada degrau de $100 \mathrm{~mm}$
\end{tabular} . . . . . . 128

Figura $37 \quad$ Erro quadrático para entrada degrau de $100 \mathrm{~mm}$. . . . . . . . . . . . . 129

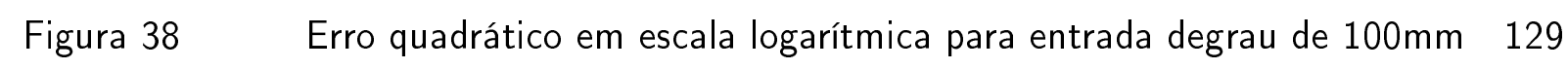

Figura $39 \quad$ Diagrama de bode para ângulo de rolagem . . . . . . . . . . . . . . 130

Figura $40 \quad$ Diagrama de bode do erro do ângulo de rolagem . . . . . . . . . . . 131

Figura $41 \quad$ Máximo valor singular da função de transferência do erro $\left(G_{e}(s)\right)$. . 131

Figura $42 \quad$ Métrica de representatividade $\mathcal{M}_{\mathcal{L}_{2}}$ dos modelos reduzidos . . . . . . 132

Figura $43 \quad$ Métrica de representatividade $\mathcal{M}_{s s}$ dos modelos reduzidos . . . . . . 133

Figura $44 \quad$ Métrica de representatividade $\mathcal{M}_{s}$ dos modelos reduzidos . . . . . . . 134

Figura $45 \quad$ Métrica de representatividade $\mathcal{M}_{\mathcal{H}_{\infty}}$ dos modelos reduzidos . . . . . . 134

Figura $46 \quad$ Métrica de representatividade $\mathcal{M}_{\mathcal{H}_{2}}$ dos modelos reduzidos . . . . . . 135

Figura $47 \quad$ Métrica de representatividade $\mathcal{M}_{\text {eqmax }}$ dos modelos reduzidos . . . . . 136

Figura $48 \quad$ Métrica de representatividade $\sigma_{\max }\left(G_{e}(\infty)\right)$ dos modelos reduzidos 136

Figura $49 \quad$ Métrica de representatividade $\mathcal{M}_{t r}$ dos modelos reduzidos . . . . . . 139

Figura $50 \quad$ Métrica de representatividade $\mathcal{M}_{\text {fr }}$ dos modelos reduzidos . . . . . . 140

Figura $51 \quad$ Métrica de representatividade $\mathcal{M}_{\text {eqmax }}$ dos modelos reduzidos . . . . . 140

Figura $52 \quad$ Autovalores do modelo completo de trem . . . . . . . . . . . . . . 141

Figura $53 \quad$ Métrica de representatividade $\mathcal{M}_{\mathcal{L}_{2}}$ em função do número de GDL reduzidos . . . . . . . . . . . . . . . . . . . . . 142

Figura $54 \quad$ Métrica de representatividade $\mathcal{M}_{\mathcal{H}_{2}}$ em função do número de GDL

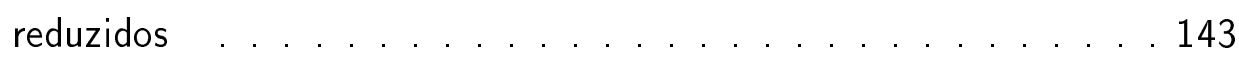


Figura 55 Métrica de representatividade $\mathcal{M}_{\mathcal{H}_{\infty}}$ em função do número de GDL reduzidos . . . . . . . . . . . . . . . . . . . . . . 145

Figura $56 \quad$ Métrica de representatividade dos modelos $\mathcal{M}_{\text {eqmax }}$ em função do número de GDL reduzidos . . . . . . . . . . . . . . . . . . 145

Figura 57 Métrica de representatividade dos modelos $\mathcal{M}_{s s}$ em função do número de GDL reduzidos . . . . . . . . . . . . . . . . . . . . . . 146

Figura 58 Representatividade total $\mathcal{R}$ em função do número de GDL reduzidos . 148

Figura 59 Representatividade total $\mathcal{R}$ em função do número de GDL reduzidos (detalhe) . . . . . . . . . . . . . . . . . . . 149

Figura 60 Disco rolando sobre um plano. . . . . . . . . . . . . . . . . 164

Figura $61 \quad$ Modelo de um quarto de veículo. . . . . . . . . . . . . 168 


\section{LISTA DE TABELAS}

Tabela 1 Exemplo da comparação entre modelos reduzidos . . . . . . . . . . . 90

Tabela 2 Exemplo da comparação entre modelos reduzidos pelo CRT . . . . . . 92

\begin{tabular}{llll}
\hline Tabela 3 & Dados de entrada do modelo de veículo de passeio $\ldots$ & $\ldots$ & $\ldots$
\end{tabular}

Tabela 4 Dados de entrada do modelo de trem . . . . . . . . . . . . . . . 100

Tabela 5 Métodos implementados . . . . . . . . . . . . . . . . . 101

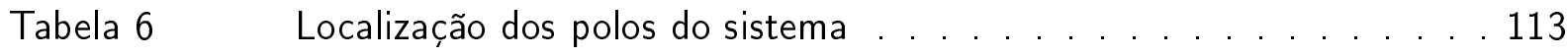

Tabela 7 Parâmetro de seleção $P_{\omega_{n}}$ baseado no critério CSF . . . . . . . . . 115

Tabela $8 \quad$ Parâmetro de seleção $P_{a f}$ baseado no critério CSFA . . . . . . . . . . 117

Tabela 9 Normas Hankel dos modos de vibrar . . . . . . . . . . . . . . . . . . 119

Tabela $10 \quad$ Parâmetro de seleção $P_{\gamma m}$ baseado no critério CSCO . . . . . . . . . 120

Tabela $11 \quad$ Parâmetro de seleção $P_{s}$ baseado no critério CSFT . . . . . . . . . . . 121

Tabela $12 \quad$ Parâmetro de seleção $P_{\sigma}$ baseado no critério CSVS . . . . . . . . . . . 123

Tabela $13 \quad$ Parâmetro de seleção $P_{\gamma}$ baseado no critério CSNH . . . . . . . . . . . 124

Tabela $14 \quad$ Norma $\left\|G_{k}\right\|_{\mathcal{H}_{\infty}}$ dos modos de vibrar do modelo completo . . . . . . 125

Tabela $15 \quad$ Representatividade dos modelos reduzidos . . . . . . . . . . . . . 137

Tabela 16 Comparação da ordem de seleção dos modos de vibrar segundo diferentes critérios . . . . . . . . . . . . . . . . . . 138

Tabela 17 Dados de entrada para o exemplo de quarto de veículo . . . . . . . 169 


\section{LISTA DE ABREVIATURAS E SIGLAS}

BFM Base de Formas Modais

BKMA Base de Krylov por método de Arnoldi

CRT

Critério da Representatividade Total

CSCO Critério de seleção baseado na observabilidade e controlabilidade

CSF Critério de seleção baseado na frequência natural

CSFA Critério de seleção baseado na frequência e fator de amortecimento

CSFT Critério de seleção baseado na função de transferêcia

CSNH Critério de seleção baseado em normas Hankel

CSVK Critério de seleção por vetores na base de Krylov

CSVS Critério de seleção baseado em valores singulares

DVS Decomposição em Valores Singulares

EDO Equações diferenciais ordinárias

EPS Eliminação por perturbação singular

EPSG Eliminação por perturbação singular generalizada

EPSGP Eliminação por perturbação singular generalizada e paramétrica

ETD Eliminação por truncamento direto

GDL Grau(s) de liberdade

$\mathrm{LCl}$

(Sistema) linear, contínuo e invariante

MIMO Sistema com múltiplas entradas e múltiplas saídas

$\mathrm{SC}$

Sistema Completo

SISO Sistema com uma entrada e uma saída

SR Sistema Reduzido 


\section{LISTA DE SÍMBOLOS}

$\|\cdot\|_{\mathcal{L}_{2}} \quad$ Norma $\mathcal{L}_{2}$ de uma função

$\|\cdot\|_{\mathcal{L}_{\infty}} \quad$ Norma $\mathcal{L}_{\infty}$ de uma função

$\|\cdot\|_{\mathcal{H}_{2}} \quad$ Norma $\mathcal{H}_{2}$ de uma função

$\|\cdot\|_{\mathcal{H}_{\infty}} \quad$ Norma $\mathcal{H}_{\infty}$ de uma função

\|. $\quad$ Norma de um vetor ou de uma matriz

$A^{*}=\bar{A}^{T} \quad$ Transposto do complexo conjugado

$A^{T} \quad$ Transposta da matriz $A$

$A^{-1} \quad$ Inversa da matriz $A$

$A B C D \quad$ Matrizes que formam o sistema de alta ordem, ou sistema completo

$A_{e} B_{e} C_{e} D_{e}$ Matrizes do sistema que calcula o erro do SR

$A_{k} B_{k} C_{k} D_{k}$ Matrizes do sistema dinâmico do k-ésimo modo de vibrar

$A_{r} B_{r} C_{r} D_{r}$ Matrizes do sistema de ordem reduzida, ou sistema reduzido

$e_{e q} \quad$ Vetor de erro do SR na condição de equilíbrio

$e_{q}(t) \quad$ Erro quadrático

$e_{q p}(t) \quad$ Erro quadrático ponderado

$F_{t} \quad$ Força longitudinal resultante do sistema de tração da locomotiva

$g(t) \quad$ Resposta do sistema ao impulso

$G(s) \quad$ Função de transferência do sistema completo

$G_{k}(s) \quad$ Função de transferência do k-ésimo modo de vibrar

$G_{r}(s) \quad$ Função de transferência do SR

$G_{r c}(s) \quad$ Função de transferência do SR e corrigido

$G_{e}(s) \quad$ Função de transferência do erro

$\gamma_{i} \quad$ i-ésima norma Hankel do sistema

$\Gamma \quad$ Matriz que contém as normas Hankel do sistema

$I_{n} \quad$ Matriz identidade de ordem $n$

$L_{c} \quad$ Gramiano de controlabilidade

$L_{o} \quad$ Gramiano de observabilidade

$L_{x} \quad$ Gramiano cruzado

$\lambda_{i}($.$) \quad i-ésimo autovalor da matriz$

$\lambda_{\max }($.$) \quad Máximo autovalor (em módulo) da matriz$

$\mathcal{M} \quad$ Vetor de métricas do modelo reduzido

$\mathcal{M}_{e q} \quad$ Métrica de comparação para o erro da resposta estática

$\mathcal{M}_{\mathcal{L}_{2}} \quad$ Métrica para a norma $\mathcal{L}_{2}$ do erro do SR

$\mathcal{M}_{\mathcal{H}_{2}} \quad$ Métrica para a norma $\mathcal{H}_{2}$ do erro do SR 


\begin{tabular}{|c|c|}
\hline $\mathcal{M}_{\mathcal{H}_{\infty}}$ & Métrica para a norma $\mathcal{H}_{\infty}$ do erro do $\mathrm{SR}$ \\
\hline $\mathcal{M}_{s}$ & Métrica para os tempos de acomodação do SR \\
\hline $\mathcal{M}_{s s}$ & Métrica de comparação para os sobre-sinais do SR \\
\hline $\operatorname{Nul}(A)$ & Núcleo (ou Kernel) da matriz $A$ \\
\hline$n$ & Ordem do sistema completo (SC) \\
\hline$n_{m}$ & Número de modos de vibrar no SC \\
\hline$n_{m r}$ & Número de modos de vibrar no SR \\
\hline$n_{0}$ & Número graus de liberdade no sistema de segunda ordem \\
\hline$n_{r}$ & Ordem do sistema reduzido (SR) \\
\hline$n_{u}$ & Número de entradas do sistema \\
\hline$n_{y}$ & Número de saídas do sistema \\
\hline$P$ & Matriz de ponderação \\
\hline$P_{\omega n}$ & Parâmetro de seleção baseado na frequência \\
\hline$P_{a f}$ & Parâmetro de seleção baseado no amortecimento e frequência \\
\hline$P_{s}$ & Parâmetro de seleção baseado na função de transferêcia \\
\hline$P_{\sigma}$ & Parâmetro de seleção baseado em valores singulares \\
\hline$P_{\gamma}$ & Parâmetro de seleção baseado nas normas Hankel do sistema \\
\hline$P_{\gamma m}$ & Parâmetro de seleção baseado nas normas Hankel dos modos de vibrar \\
\hline $\mathbb{R}$ & Conjunto dos números reais \\
\hline $\mathbb{R}^{+}$ & Conjunto dos números reais não negativos \\
\hline $\mathcal{R}$ & Representatividade de um modelo reduzido \\
\hline$r$ & Número de graus de liberdade eliminados do SC \\
\hline $\operatorname{span} C o l(A)$ & Espaço formado pelas colunas linearmente independentes da matriz $A$ \\
\hline$\sigma_{i}()$. & i-ésimo valor singular da matriz \\
\hline$\sigma_{\max }()$. & Máximo valor singular da matriz \\
\hline $\operatorname{tr}[A]$ & Traço da matriz (soma dos valores da diagonal principal) \\
\hline$u_{d}$ & Entrada degrau em um sistema \\
\hline$v \cdot u$ & Produto escalar do vetor $v$ com o vetor $u$ \\
\hline$x_{e q}$ & Vetor de estados do SC na condição de equilíbrio \\
\hline$x_{r e q}$ & Vetor de estados do SR na condição de equilíbrio \\
\hline$x_{r}$ & Vetor de estados do SR \\
\hline$y$ & Vetor de resposta do SC \\
\hline$y_{i}$ & i-ésima posição do vetor de resposta do SC \\
\hline$y_{e q}$ & Vetor de resposta do SC na condição de equilíbrio \\
\hline$y_{\text {eqi }}$ & i-ésimo valor do vetor vetor $y_{e q}$ \\
\hline$y_{r}$ & Vetor de resposta do SR \\
\hline
\end{tabular}




$\begin{array}{ll}y_{r i} & \text { i-ésima posição do vetor de resposta do SR } \\ y_{r e q} & \text { Vetor de resposta do SR na condição de equilíbrio } \\ y_{r e q i} & \text { i-ésimo valor do vetor } y_{r e q} \\ y_{r c e q} & \text { Vetor de resposta do SR corrigido na condição de equilíbrio } \\ y_{s s i} & \text { Máximo sobre-sinal da i-ésima saída do SC } \\ y_{r s s i} & \text { Máximo sobre-sinal da i-ésima saída do SR } \\ \zeta_{k} & \text { Fator de amortecimento do k-ésimo modo de vibrar } \\ \omega_{n k} & \text { Frequência natural não amortecida do k-ésimo modo de vibrar } \\ \omega_{d k} & \text { Frequência natural amortecida do k-ésimo modo de vibrar }\end{array}$




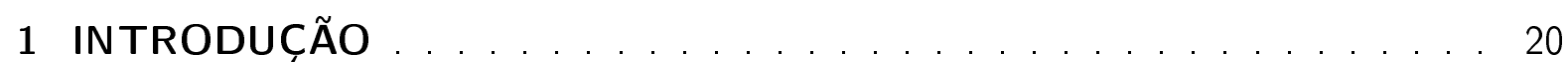

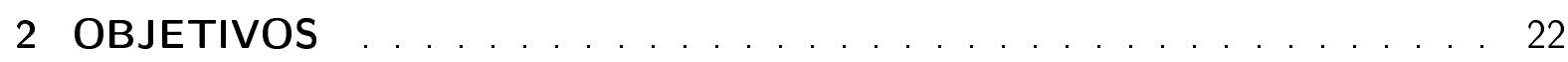

3 REVISÃO BIBLIOGRÁFICA . . . . . . . . . . . . . 23

4 REDUÇÃOO DA ORDEM DE SISTEMAS DINÂMICOS . . . . . . . . . . 27

4.1 METODOLOGIA PROPOSTA PELO AUTOR PARA REDUÇÃO DA ORDEM DE

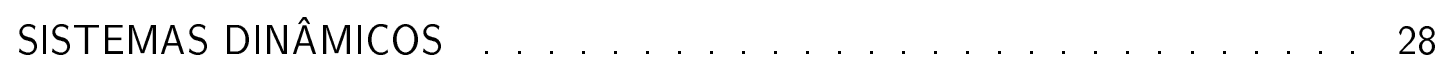

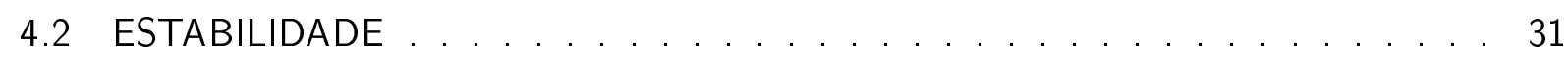

4.3 CONTROLABILIDADE E OBSERVABILIDADE $\ldots \ldots \ldots \ldots . \ldots 31$

4.4 RESPOSTA EM ESTADO ESTÁVEL . . . . . . . . . . . . . . . . . . . . . . . . 34

4.4 .1 Resposta em altas frequências $\ldots \ldots \ldots \ldots \ldots$

4.4 .2 Resposta estática . . . . . . . . . . . . . . . . . . . 35

4.5 CONSIDERAÇÕES SOBRE CUSTO COMPUTACIONAL ......... . . 36

5 MUDANÇAS DE BASE . . . . . . . . . . . . . . . 37

$5.1 \quad$ BASE DE FORMAS MODAIS (BFM) $\ldots \ldots \ldots \ldots$

5.1 .1 Vibração livre . . . . . . . . . . . . . . . . . . . . . . 41

5.1 .2 Resposta em frequência . . . . . . . . . . . . . . . . . 43

5.2 BASE DE VETORES SINGULARES . . . . . . . . . . . . . . . 45

5.3 ESPAÇO DE SÉRIES DE POTÊNCIA (BASE DE KRYLOV). . . . . . . . . . 48

5.3.1 Base ortonormal de séries de potência (Método de Arnoldi) . . . . 50

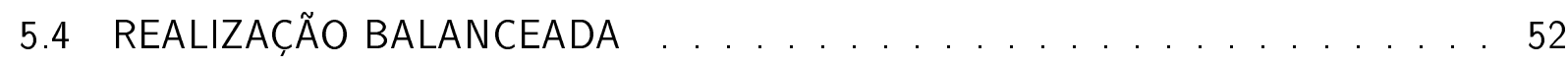

6 CRITÉRIOS DE SELEÇÃO . . . . . . . . . . . . . . . . . . . . . 55

6.1 CRITÉRIOS DE SELEÇÃO EXCLUSIVOS PARA BASE MODAL . . . . . . . . 55

6.1 .1 Critério de seleção baseado na frequência natural (CSF) . . . . . . . 56

6.1.2 Critério de seleção baseado na frequência natural e fator de amorte-

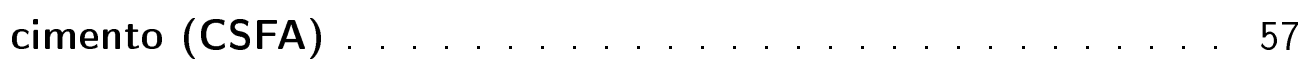

6.1.3 Critério de seleção baseado na controlabilidade e observabilidade (CSCO) 58

6.1.4 Critério de seleção baseado na função de transferêcia (CSFT) . . . . . 59

6.2 CRITÉRIO DE SELEÇÃO BASEADO EM VALORES SINGULARES (CSVS) . . . 61

6.3 CRITÉRIO DE SELEÇÃO POR VETORES NA BASE DE KRYLOV (CSVK) . . . 61

6.4 CRITÉRIO DE SELEÇÃO BASEADO EM NORMAS HANKEL (CSNH) . . . . . 62

7 MÉTODOS DE ELIMINAÇÃO . . . . . . . . . . . . . . . . . . . . 64 
7.1 ELIMINAÇÃO POR CONDENSAÇÃO ESTÁTICA . . . . . . . . . . . . . . 64

7.1 .1 Eliminação por método de Guyan . . . . . . . . . . . . . . 65

7.1 .2 Eliminação por método de Guyan Modificado . . . . . . . . . . . 67

7.2 ELIMINAÇÃO POR TRUNCAMENTO DIRETO (ETD) . . . . . . . . . . . 68

7.3 ELIMINAÇÃO POR MÉTODOS DE PERTURBAÇÃO . . . . . . . . . . . . 70

7.3.1 Eliminação por perturbação singular (EPS) . . . . . . . . . . 71

7.3 .2 Eliminação por perturbação singular generalizada (EPSG) ‥ . . 72

7.3.3 Eliminação por perturbação singular generalizada e paramétrica (EPSGP) 73

8 CORREÇÕES DE MODELOS REDUZIDOS . . . . . . . . . . . . 75

8.1 RESPOSTA EM UMA FREQUÊNCIA DETERMINADA . . . . . . . . . . . 75

8.1 .1 Correção de $G_{r}\left(s_{0}\right)$ através de $D_{r} \ldots \ldots \ldots \ldots \ldots \ldots$

8.1 .2 Correção de $G_{r}\left(s_{0}\right)$ através de $C_{r} \ldots \ldots \ldots \ldots \ldots$

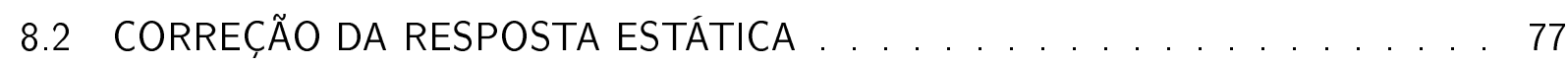

8.3 CORREÇÃO DO MÁXIMO SOBRE-SINAL . . . . . . . . . . . . . . . . . . . . 79

9 REPRESENTATIVIDADE DE MODELOS REDUZIDOS … . . . . . 81

9.1 MÉTRICAS DA RESPOSTA NO DOMíNIO DO TEMPO . . . . . . . . . . 81

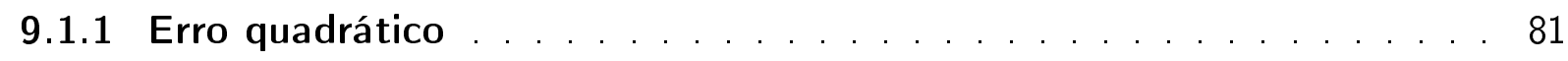

9.1 .2 Norma $\mathcal{L}_{2}$ do erro $\ldots \ldots \ldots \ldots \ldots$

9.1 .3 Máximo sobre-sinal . . . . . . . . . . . . . . . . . . . 83

9.1 .4 Tempo de acomodação $\ldots \ldots \ldots$. . . . . . . . . . . . 84

9.2 MÉTRICAS DA RESPOSTA NO DOMíNIO DA FREQUÊNCIA . . . . . . . . 85

9.2 .1 Norma $\mathcal{H}_{2}$ da função de transferência . . . . . . . . . . . . . 86

9.2 .2 Norma $\mathcal{H}_{\infty}$ da função de transferência . . . . . . . . . . 87

9.2 .3 Maior valor singular da função de transferência . . . . . . . . . . 88

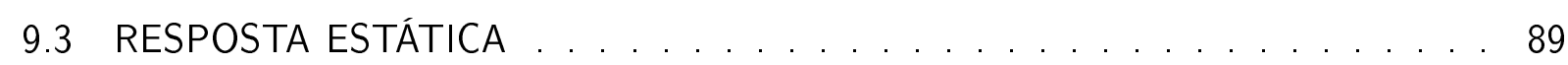

9.4 LIMITAÇÕES DAS TÉCNICAS APRESENTADAS . . . . . . . . . . . . . . 89

9.5 NOVA PROPOSTA PARA QUANTIFICAR A REPRESENTATIVIDADE DE UM MODELO REDUZIDO . . . . . . . . . . . . . . . . . 91

10 ESTUDOS DE CASO . . . . . . . . . . . . . . . . . . . . . 93

10.1 MODELO PARA ESTUDO DA DINÂMICA VERTICAL DE VEÍCULOS DE PASSEIO 93 10.2 MODELO PARA ESTUDO DA DINÂMICA LONGITUDINAL DE UM TREM. . . 98

11 TÉCNICAS DE REDUÇÃO IMPLEMENTADAS . . . . . . . . . . . . 101

11.1 TÉCNICA R1 . . . . . . . . . . . . . . . . . . . . . . . . 102

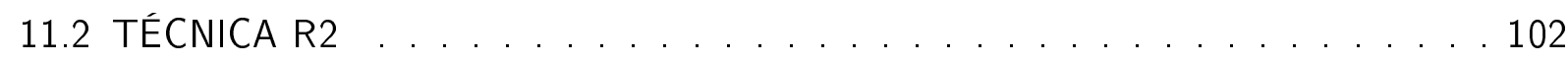


11.3 TÉCNICA R3 $\ldots \ldots \ldots \ldots \ldots \ldots \ldots$

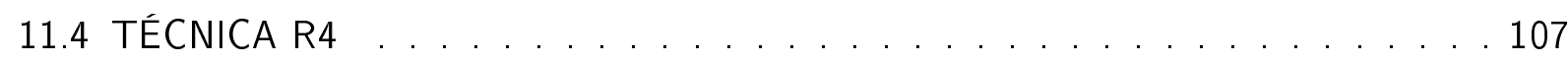

11.5 TÉCNICA R5 $\ldots \ldots \ldots \ldots \ldots \ldots$

11.6 TÉCNICA R6 $\ldots \ldots \ldots \ldots . \ldots \ldots$

11.7 TÉCNICA R7 . . . . . . . . . . . . . . . . . . . . . . . 110

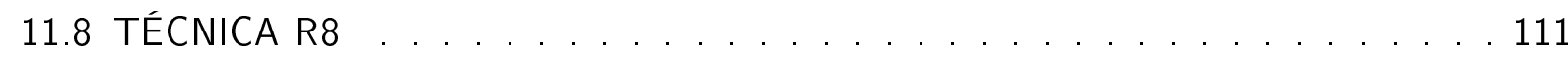

12 RESULTADOS . . . . . . . . . . . . . . . . . . . . . . . . . 112

12.1 PROPRIEDADES DO MODELO COMPLETO DE VEíCULO DE PASSEIO . . . . 112

12.2 RESULTADOS DOS CRITÉRIOS DE SELECCÃO DE GDL . . . . . . . . . . 115

12.2.1 Seleção de GDL segundo o critério CSF . . . . . . . . . . . . 115

12.2.2 Seleção de GDL segundo o critério CSFA . . . . . . . . . . 116

12.2.3 Seleção de GDL segundo o critério CSCO . . . . . . . . . . . . 118

12.2.4 Seleção de GDL segundo o critério CSFT . . . . . . . . . . . . . 120

12.2.5 Seleção de GDL segundo o critério CSVS . . . . . . . . . . . . . 122

12.2.6 Seleção de GDL segundo o critério CSNH . . . . . . . . . . . . . 122

12.3 RESULTADOS COMPARATIVOS . . . . . . . . . . . . . . . . . . . 125

12.3.1 Cálculo do número de GDL eliminados . . . . . . . . . . . . . 125

12.3.2 Resultados no domínio do tempo . . . . . . . . . . . . . 127

12.3 .3 Resultados no domínio da frequência . . . . . . . . . . . . . 130

12.3.4 Resultados por métricas de representatividade . . . . . . . . . . . 132

12.3.5 Cálculo da representatividade total dos modelos reduzidos . . . . . . 136

12.4 INFLUÊNCIA DO CRITERIO DE SELEÇÃO NA RESPOSTA DO MODELO REDUZIDO UTILIZANDO O MODELO DE VEÍCULO DE PASSEIO . . . . . . . 138

12.5 INFLUÊNCIA DO NÚMERO DE GDL REDUZIDOS UTILIZANDO O MODELO DE TREM . . . . . . . . . . . . . . . . . . . . . . . . 141

12.5.1 Resultados por metricas de representatividade . . . . . . . . . . . . 142

12.5.2 Representatividade total dos modelos reduzidos . . . . . . . . . . . 147

12.5.3 Seleção da técnica mais adequada para a redução do modelo de trem 149

13 DISCUSSỖES DOS RESULTADOS . . . . . . . . . . . . . . . . . . 151

14 CONCLUSÕES . . . . . . . . . . . . . . . . . . . 153

15 TRABALHO FUTURO . . . . . . . . . . . . . . . . . . 155

Referências . . . . . . . . . . . . . . . . . . . . . 156

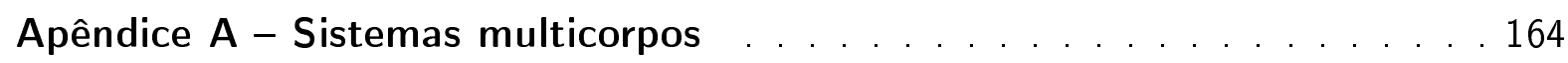


A.1 MÉTODO DE LAGRANGE . . . . . . . . . . . . . . . . . . . . . . . . . . 165

A.2 EXEMPLO DE APLICAÇÃO DO MÉTODO DE LAGRANGE . . . . . . . . . . 167

Apêndice B - Normas de vetores, matrizes e funções . . . . . . . . . . . 170

B.1 NORMAS INDUZIDAS . . . . . . . . . . . . . . . . . . 170

B.2 NORMAS NÃO INDUZIDAS . . . . . . . . . . . . . . . . . . 170

B.3 NORMAS DE FUNCCÕES . . . . . . . . . . . . . . . . . . . . 171

B.4 CORRELAÇÃO ENTRE NORMAS INDUZIDAS E NÃO INDUZIDAS . . . . . . . 172

B.5 CORRELAÇÃO ENTRE NORMAS NO DOMÍNIO DO TEMPO E DA FREQUÊNCIA 172

B.6 CORRELAÇÃO ENTRE NORMAS NOS ESPAÇOS $\mathcal{L}$ E H . . . . . . . . 173

Apêndice C - Exemplos numéricos . . . . . . . . . . . . . . . . . . . . 174

C.1 EXEMPLO NUMÉRICO: DECOMPOSICCÃO EM VALORES SINGULARES . . . 174

C.2 EXEMPLO NUMÉRICO: ESPAÇO ORTOGONAL DE SÉRIES DE POTÊNCIA . . 175

C.3 EXEMPLO NUMÉRICO: MÉTODO DE GUYAN E MÉTODO DE GUYAN MODIFICADO . . . . . . . . . . . . . . . . . . . . 176

C.4 EXEMPLO NUMÉRICO: CORREÇÃO DA RESPOSTA ESTÁTICA DE UM SR . . 177 


\section{INTRODUÇÃO}

A utilização de modelos numéricos que representem fenômenos reais sempre foi muito importante para diversos setores da engenharia. Estes modelos auxiliam no desenvolvimento de novas tecnologias, equipamentos, materiais e processos, assim como a simulação de seu comportamento.

Com o advento da tecnologia em computadores, o poder de processamento e armazenamento computacional aumentou consideravelmente nas últimas décadas. Junto a esta tendência, observa-se a demanda pelo aumento da complexidade de modelos numéricos, o que pode ser indesejado em muitas aplicações. Além disso, é sabido que o custo computacional e o tempo de pré-processamento desses modelos ainda é um fator decisivo em processos que envolvem:

- Simulação em tempo real

- Otimização

- Processamento embarcado

- Ciclos de projeto com prazos de curta duração

- Projeto de controladores

- Estudo da estabilidade e robustez de sistemas

- Compressão de imagens

Durante o processo de modelagem de sistemas, espera-se encontrar modelos que sejam representativos em relação aos fenômenos estudados. Desta maneira, as técnicas utilizadas na modelagem são um fator crítico para que consigam prever fielmente o comportamento do sistema em questão.

Escolher o nível de detalhamento mais adequado para um modelo depende de fatores como a natureza do sistema, dos fenômenos a serem observados, do tipo de análise que será realizada, da infra-estrutura de processamento disponível, tempo disponível para realizar o processamento, etc. Além disso, o detalhamento excessivo de modelos pode considerar fenômenos que dificultam o entendimento e análise de resultados.

Desta maneira, deseja-se encontrar modelos numéricos de baixo custo computacional que representem as características principais de um fenômeno real.

Para aplicações de projetos de controladores, a simplicidade na modelagem também pode levar a controladores de menor ordem, que são mais simples de implementar e sintonizar (ERSAL et al., 2008). 
O projeto de controladores mais sofisticados, por exemplo o robusto $H_{\infty}$, tem sido cada vez mais frequente para sistemas de alta ordem. Entretanto, com a aplicação destas técnicas de controle, a ordem do controlador é normalmente similar ou igual à do sistema controlado (que por sua vez é de alta ordem). Isso pode trazer uma série de dificuldades de implementação, como a necessidades de atuadores de alta potência, problemas numéricos no tratamento de sinais e excessiva ocupação de hardware (OBINATA; ANDERSON, 2001).

Modelos reduzidos também viabilizam o estudo da estabilidade e robustez de sistemas dinâmicos não lineares além da detecção de bacias de atração. Sua utilização também possibilita a detecção de possíveis bifurcações dinâmicas, que por requerer bastante esforço algébrico, são pouco viáveis para sistemas de alta ordem (NETO, 1998).

As metodologias de redução da ordem de sistemas que serão apresentadas neste trabalho podem ser utilizadas nos mais diversos setores da engenharia, possibilitando a reprodução das principais características de modelos de alta ordem com custos reduzidos de processamento e armazenagem computacional. 


\section{OBJETIVOS}

O objetivo deste estudo é apresentar uma revisão sobre técnicas de redução da ordem de modelos dinâmicos lineares e invariantes no tempo. Com a implementação de tais técnicas, o autor mostra que é possível reproduzir as principais características da resposta de um modelo de alta ordem através de um modelo de ordem reduzida com menor número de graus de liberdade.

Também objetiva-se neste trabalho introduzir contribuições ao propor novas métricas de representatividade para modelos reduzidos. Além disso, objetiva-se propor uma nova definição para o desempenho de um sistema reduzido e uma técnica de quantificar o desempenho do mesmo. 


\section{REVISÃO BIBLIOGRÁFICA}

Barbosa (BARBOSA; COSTA, 1996) deduziu, utilizando técnica de multicorpos, um modelo de escala reduzida que possibilitou o estudo da dinâmica de rodeiros ferroviários. Com isso o autor mostrou uma metodologia que possibilita calcular, com custo computacional reduzido, o limite de estabilidade do sistema e suas características dinâmicas mais significativas.

O projeto de veículos e a implementação de algortimos de controle nos sistemas de tração, frenagem, suspensão, transmissão, direção, etc tem levado à utilização de modelos numéricos de alta ordem (CHERRY; COSTA; JONES, 1995), o que pode ser indesejado. Esforços foram realizados para a obtenção de modelos numéricos reduzidos para simulação de sistemas veiculares como em (COSTA, 1991), (COSTA; JONES, 1992), (COSTA; JONES, 1995) e (COSTA, 1992).

Alleyene (ALLEYNE, 1997) modelou um veículo em três níveis de detalhamento distintos para estudos de frenagem com sistemas ABS e de suspensões ativas. Estas diferentes configurações ajudam a entender a influência e o impacto que modelos mais completos têm na análise do sistema e na avaliação de seu desempenho.

Aplicações do método de Guyan para eliminação de graus de liberdade são amplamente encontradas na literatura. No campo da simulação de veículos através da técnica de multicorpos, Lee (LEE et al., 2005) construiu um modelo para estudo da interação entre um veículo monotrilho e a estrutura que o sustenta. O número de GDL da estrutura foi reduzido utilizando método de Guyan para reduzir o custo computacional do modelo.

Ersal (ERSAL et al., 2008) apresenta uma compilação de métodos que podem ser usados para a simulação de sistemas dinâmicos. O autor descreve técnicas baseadas no domínio da frequência, espaço de estados, otimização e energia.

Besselink (BESSELINK et al., 2013) apresentou uma revisão sobre técnicas para redução da ordem de sistemas dinâmicos lineares. Vantagens e desvantagens entre as técnicas são apresentadas e comparadas de maneira qualitativa e quantitativa.

O método denominado análise de importância descrito em (ERSAL et al., 2009) tem a vantagem de preservar o significado das variáveis de estado de um modelo completo em seu equivalente reduzido. A técnica pode ser aplicada em análises não lineares, dinâmicas ou cinemáticas.

Diferentes abordagens para reduções realizadas na base de Krylov foram estudadas por Bai (BAl, 2002) considerando o custo computacional para redução da ordem do sistema e erro do modelo reduzido em estado estável e em resposta transiente.

Esforços tem sido feitos no desenvolvimento de métodos de redução baseados na projeção do sistema em uma base de Krylov, onde se busca aumentar a quantidade de momentos que 
o sistema reduzido tem em comum com o sistema completo (SALIMBAHRAMI; LOHMANN, 2006). Esta abordagem aumenta a similaridade da resposta em frequência para uma mesma quantidade de variáveis eliminadas no processo de redução.

Variações do método de redução utilizando base de Krylov descrito neste trabalho foram explorados por diversos autores, tais como aproximação de Padé via Lanczos (FELDMANN; FREUND, 1995), interpolação racional em múltiplos pontos (GRIMME, 1997) e duplo Arnoldi implicitamente reiniciado (JAIMOUKHA; KASENALLY, 1997).

Kabamba mostra um critério de seleção na base de realização balanceada. Este critério é baseado em parâmetros propostos pelo autor, que estão relacionados com ganhos do sistema associado a cada grau de liberdade (GDL). Com este criterio, o autor calcula um limite superior para a norma $\mathcal{L}_{2}$ da resposta do sistema ao impulso. (KABAMBA, 1985)

Vargas e Anderson (VARGA; ANDERSON, 2001) descrevem dois métodos de redução que são vertentes dos métodos de truncagem direta e Eliminação por Perturbação Singular aplicados à base balanceada. Foi realizada uma mudança para uma base balanceada utilizando matrizes de ponderação para ajustar o erro da resposta do sistema reduzido em duas frequências previamente determinadas.

Esforços foram realizados para desenvolver métodos que auxiliem a avaliar a capacidade que um sistema tem de ser reduzido sem que sua resposta seja consideravelmente comprometida (ANTOULAS; SORENSEN; GUGERCIN, 2001) e (ANTOULAS, 2005). Mostrou-se que taxa de decaimento das normas Hankel do sistema está associada com o número de GDL a serem eliminados e com o erro do modelo reduzido. Ou seja, quanto maior a taxa que as normas Hankel descrescem, maior a capacidade de se eliminar mais graus de liberdade, garantindo valores menores de erro do modelo simplificado.

Antoulas et al. (ANTOULAS; SORENSEN; GUGERCIN, 2001) apresentam um método para cálculo da Realização Aproximadamente Balanceada. Este método consiste em calcular diretamente um sistema reduzido aproximadamente balanceado sem que seja necessária a projeção do sistema de alta ordem na base balanceada e posterior truncamento do mesmo. Este método pode reduzir drasticamente o custo computacional em casos de sistemas de alta ordem.

Métodos de redução baseados na separação do sistema completo em subsistemas de menor ordem foram propostos inicialmente por Craig e Bampton (BAMPTON; CRAIG JR, 1968). Outros trabalhos também se baseiam na mesma metodologia, os quais são: (JR; CHANG, 1968), (CRAIG, 1995), (CRAIG, 2000) e (RIXEN, 2004).

A dedução e redução do modelo não linear de quarto de veículo com uma suspensão Macpherson de dois graus de liberdade foi abordada em (FALLAH; BHAT; XIE, 2009) e (HONG; JEON; SOHN, 1999). Os modelos propostos nestes trabalhos conseguem descrever 
a dinâmica vertical e a cinemática do sistema com maior representatividade que outros modelos lineares convencionais de quarto de veículo. Howitt e Luus (HOWITT; LUUS, 1990) mostram uma alternativa para minimizar a integral do quadrado do erro entre resultados dos modelos completo e simplificado.

Em um trabalho similar, Luus (LUUS, 1980) técnicas de redução visando minimizar a integral do quadrado do erro de modelos reduzidos submetidos a entradas tipo degrau ou impulso.

Megretski (MEGRETSKI, 2006) propôs um método de redução para realizar otimizações em sistemas estáticos lineares. Também ressalta que em aplicações onde se deseja modelar estruturas utilizando métodos de discretização, deve-se escolher com cautela número de graus de liberdade a serem eliminados do modelo completo. Essa preocupação é importante para que se represente corretamente o comportamento da estrutura, tendo em vista que a mesma possui infinitos graus de liberdade.

Givoli (GIVOLI; BARBONE; PATLASHENKO, 2004) desenvolveu um critério de seleção de modos de vibrar para redução da ordem de sistemas dinâmicos lineares projetados na base modal para simulação estrutural. Este critério é baseado em um algoritmo de otimização que minimiza a norma do vetor de forças internas na interface entre a região de interesse e a região que será reduzida.

Gugercin et al. propuseram um novo método iterativo para a redução de sistemas dinâmicos lineares que minimiza a norma $\mathcal{H}_{2}$ do erro. Entretanto, o método apresentado prova que a solução é ótima apenas localmente. Os autores também mostram que o método proposto é equivalente às duas vertentes que existiam anteriormente para a solução do problema de minimização da norma $\mathcal{H}_{2}$. (GUGERCIN; ANTOULAS; BEATTIE, 2008)

Gugercin e Antoulas mostram diversos métodos de projeção para bases balanceadas: balanceamento por Lyapunov, balanceamento estocástico, balanceamento real com restrições, balanceamento real positivo e um novo balanceamento ponderado em um intervalo de frequências (GUGERCIN; ANTOULAS, 2004). Os limites superiores da norma $\mathcal{H}_{\infty}$ do erro associado à estas técnicas são descritos pelos autores.

Varga e Parrilo propuseram um método que minimiza a norma $\mathcal{H}_{\infty}$ da função de transferência do erro de modelos reduzidos lineares (contínuos e discretos). Os modelos reduzidos são parametrizados de maneira arbitrária definida pelo analista e posteriormente o problema de otimização é resolvido. (VARGA; PARRILO, 2001)

Critérios de seleção de GDL baseados na controlabilidade e observabilidade dos modos de vibrar de um sistema foram estudados para estruturas fracamente amortecidas com polos repetidos ou com frequências naturais muito próximas. (WILLIAMS; CHENG, 1999)

Métodos de projeção em modos de vibração não lineares foram propostos por Mazzilli utili- 
zando o método das múltiplas escalas (MAZZILLI; NETO, 2002) e das variedades invariantes (MAZZILLI; SOARES; NETO, 2004) e (MAZZILLI; MONTICELLI; NETO, 2011). Os métodos foram aplicados a sistemas dinâmicos não conservativos com muitos graus de liberdade e apresentaram resultados mais próximos aos do sistema completo, quando comparados à técnicas que utilizam modos lineares obtidos através da linearização do sistema original.

Neto (NETO, 1998) apresentou um método de redução de graus de liberdade aplicado à estruturas não lineares para análise dinâmica. O autor utilizou a técnica de sobreposição de modos de vibração não lineares, que definem um campo não linear de deslocamentos da estrutura.

Rega e Trogger apresentam uma revisão detalhada de métodos para se obter modelos não lineares de ordem reduzida. No trabalho são apresentadas descrições dos seguintes métodos: método das variedades inerciais, métodos de Galerkin, decomposição ortogonal própria e método das variedades centrais. (REGA; TROGER, 2005)

Métodos de Galerkin aplicados a sistemas não lineares contínuos (ou com infinitos GDL) são discutidos e comparados por Steindl e Troger (STEINDL; TROGER, 2001). As técnicas são implementadas em um sistema com deslocamentos de grande amplitude para que os efeitos não lineares sejam mais evidentes.

Imran et al (IMRAN; GHAFOOR; SREERAM, 2014) propuseram um método de redução de sistemas dinâmicos lineares que prioriza o bom comportamento do modelo reduzido em um determinado intervalo de frequências. Os sistemas reduzidos por este método preservam a estabilidade do modelo completo.

Rahrivani et. al. propuseram uma nova métrica de seleção de GDL que são bons candidatos a serem eliminados para a base modal buscando reduzir a norma $\mathcal{H}_{2}$ do erro do modelo reduzido. Esta métrica basea-se na hipótese de que o sistema seja fracamente amortecido e calcula um parâmetro de seleção que quantifica a contribuição de cada modo de vibrar na norma $\mathcal{H}_{2}$ do sistema completo. (RAHROVANI; VAKILZADEH; ABRAHAMSSON, 2014)

Fehr e Eberhard (FEHR; EBERHARD, 2011) apresentam uma metodologia para simulação de sistemas multicorpos com corpos flexíveis. Nesta metodologia, são implementados métodos de redução no sistema descrito em equações de segunda ordem. Os autores implementaram e compararam os resultados obtidos por reduções nas bases de Krylov, balanceada e modal.

Fischer e Eberhard (FISCHER; EBERHARD, 2014) realizaram análises comparativas sobre dois métodos de redução utilizados para simular sistemas dinâmicos multicorpos com comportamento flexível. Os componentes flexíveis foram modelados utilizando técnica de elementos finitos e posteriormente os respectivos modelos reduzidos foram calculados através dos métodos de Krylov e Decomposição Ortogonal Própria. 


\section{REDUÇÃO DA ORDEM DE SISTEMAS DINÂMICOS}

Neste capítulo, serão apresentados conceitos básicos sobre a redução da ordem de sistemas dinâmicos lineares contínuos e invariantes lineares, contínuos e invariantes no tempo (LCI), assim como métodos de análise desses sistemas que possibilitam determinar propriedades e o comportamento dos mesmos.

Dado um sistema de ordem $n$, com $x \in \mathbb{R}^{n}$, com $n_{u}$ entradas e $n_{y}$ saídas, neste trabalho chamado de sistema de alta ordem ou sistema completo (SC)

$$
\left\{\begin{array}{l}
\dot{x}=A x+B u \\
y=C x+D u
\end{array}\right.
$$

que também pode ser representado na forma de espaço de estados onde a dimensão das matrizes $A, B, C$ e $D$ são $n \times n, n \times n_{u}, n_{y} \times n$ e $n_{y} \times n_{u}$ respectivamente.

$$
\left\{\begin{array}{l}
\dot{x} \\
\hline y
\end{array}\right\}=\left[\begin{array}{l|l}
A & B \\
\hline C & D
\end{array}\right]\left\{\begin{array}{l}
x \\
\hline u
\end{array}\right\}
$$

Deseja-se encontrar uma aproximação de ordem reduzida do sistema completo, com $r$ GDL eliminados e cuja ordem seja $n_{r}=n-r$ e $x_{r} \in \mathbb{R}^{n_{r}}$. A dimensão das matrizes $A_{r}, B_{r}, C_{r}$ e $D_{r}$ são $n_{r} \times n_{r}, n_{r} \times n_{u}, n_{y} \times n_{r}$ e $n_{y} \times n_{u}$ respectivamente.

$$
\left\{\begin{array}{c}
\dot{x}_{r} \\
\hline y_{r}
\end{array}\right\}=\left[\begin{array}{c|c}
A_{r} & B_{r} \\
\hline C_{r} & D_{r}
\end{array}\right]\left\{\begin{array}{c}
x_{r} \\
\hline u_{r}
\end{array}\right\}
$$

Neste trabalho, o sistema de equações 3 será chamado de sistema de ordem reduzida ou sistema reduzido $(\mathrm{SR})$

Portanto o número entradas e saídas dos sistemas completo e reduzido permanecem iguais.

Vale lembrar que para o caso particular onde $n_{u}=n_{y}=1$, o sistema é chamado de SISO, caso contrário, é chamado de MIMO.

Tanto a resposta $y_{r}$ do quanto o vetor de estados $x_{r}$ na equação 3 são respectivamente uma aproximação de $y$ e $x$ do sistema completo.

A diferença entre as respostas do sistema de ordem completa e do sistema de ordem reduzida $e(t)=y(t)-y_{r}(t)$, também chamado erro do sistema reduzido, é:

$$
\left[\begin{array}{cc|c}
A & 0 & B \\
0 & A_{r} & B_{r} \\
\hline C & -C_{r} & D-D_{r}
\end{array}\right]\left\{\begin{array}{c}
x \\
x_{r} \\
\hline u
\end{array}\right\}=\left\{\begin{array}{c}
\dot{x} \\
\dot{x}_{r} \\
\hline e
\end{array}\right\}
$$




\subsection{METODOLOGIA PROPOSTA PELO AUTOR PARA REDUÇÃO DA ORDEM DE SIS- TEMAS DINÂMICOS}

Neste trabalho, o autor propõe uma metodologia para a redução da ordem de um sistema dinâmico, mostrada na figura 1. A metodologia proposta consiste em implementar quatro processos principais, que são:

- Mudança de base (processo opcional durante a redução)

- Escolha e implementação do critério para seleção dos GDL que serão eliminados

- Escolha e implementação de um método de eliminação

- Implementar correções do modelo reduzido (processo opcional durante a redução)

Na metodologia de redução proposta, o modelo completo é projetado em uma base que favorece a interpretação de determinadas propriedades do sistema dinâmico. Através de um critério de seleção, os GDL do sistema são reorganizados na ordem do mais dominante para o menos dominante segundo o critério escolhido. O número de GDL a serem reduzidos é determinado e os mesmos são eliminados através de um método de eliminação. Após a eliminação dos GDL, o analista já possui um modelo de ordem reduzida. Entretanto fica a seu critério aplicar métodos de correção da resposta do sistema reduzido.

Como alguns critérios de seleção e de eliminação dos GDL dependem de interpretações de propriedades do sistema, a base em que o mesmo está descrito é determinante para que os mesmos sejam aplicados. No capítulo 5 será apresentada uma seleção de bases usadas para redução da ordem de sistemas. Vale lembrar que este processo é opcional e o analista pode decidir manter o sistema na base em que se encontra.

Após a mudança de base, no capítulo 6 são descritos critérios que auxiliam na escolha dos graus de liberdade que serão eliminados do sistema. Para cada critério, é estabelecido um parâmetro de seleção que atribui um valor escalar a cada GDL. Este parâmetro quantifica a importância de se preservar este GDL no modelo reduzido.

Após a determinação dos parâmetros de seleção, o vetor de estados do sistema completo deve ser rearranjado de maneira que os valores do parâmetro de seleção estejam em ordem decrescente. Portanto os primeiros GDL no vetor de estados rearranjado serão bons candidatos a serem preservados e os últimos serão bons candidatos a serem eliminados.

Após o rearranjo do vetor de estados do sistema completo e antes da eliminação dos GDL, algumas técnicas de redução permitem que o analista possa calcular métricas que quantificam o erro associado a determinadas características dinâmicas do sistema reduzido em função do número de GDL eliminados. Estas técnicas serão apresentadas no capítulo 11 . 
Figura 1 - Fluxograma para redução da ordem de modelos

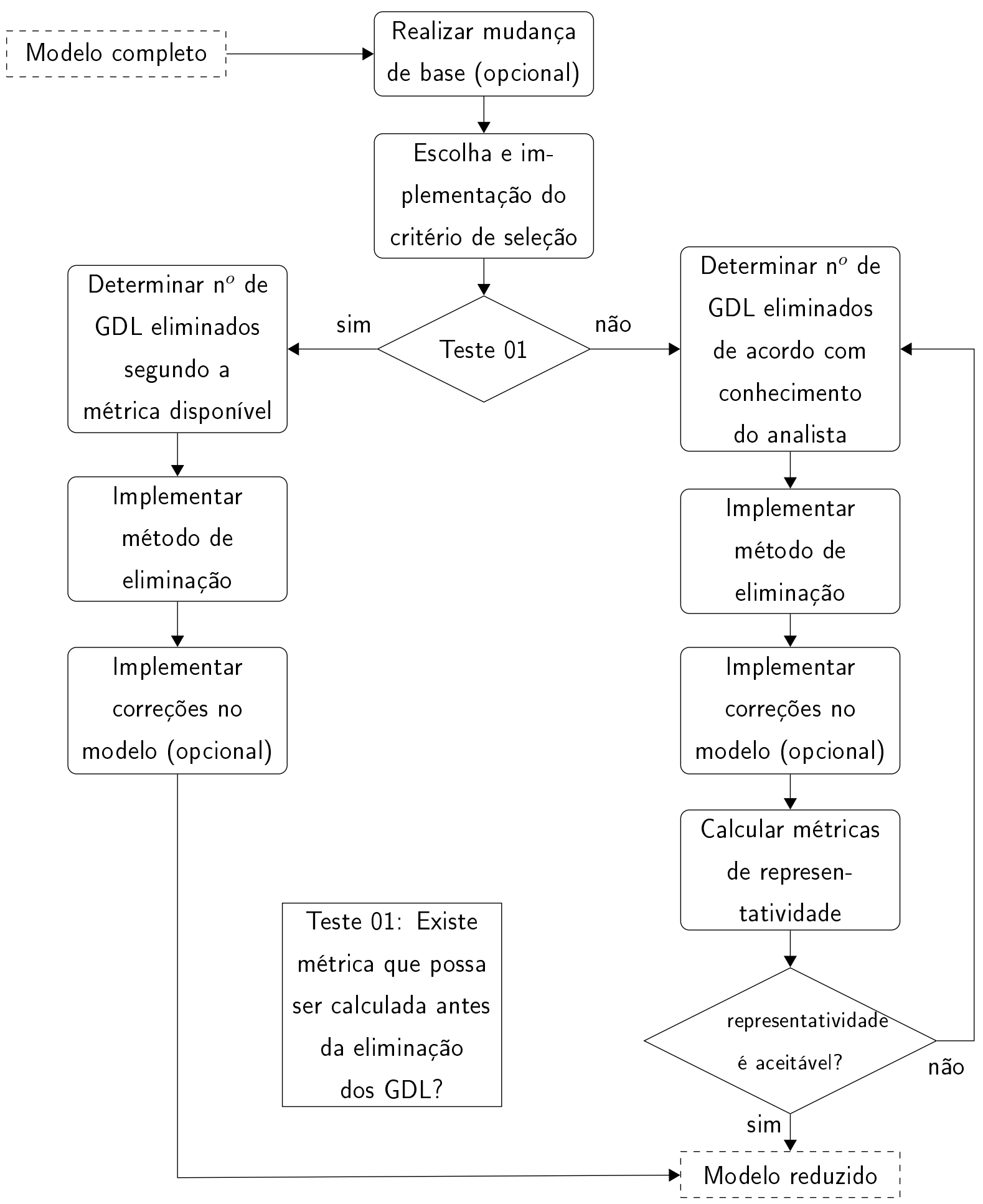

Caso seja possível calcular uma métrica de erro em função da quantidade de GDL eliminados, o analista pode determinar o número de variáveis que serão reduzidas, antecipando parte do comportamento do sistema reduzido através desta métrica. Esta situação é descrita pela parte esquerda do fluxograma mostrado na figura 1. 
Caso contrário, o analista deverá propor um número de GDL a serem eliminados. As métricas de representatividade deverão ser calculadas apenas depois da eliminação dos GDL e da correção do modelo. Caso as métricas não tenham valores dentro da faixa esperada, o analista deve mudar o número de GDL a serem eliminados e repetir o processo até que alcance valores aceitáveis para as mesmas. Esta situação é descrita pela parte direita do fluxograma mostrado na figura 1 .

Após o rearranjo do vetor de estados e seleção dos GDL que serão eliminados, pode-se reescrever a equação 2 na forma abaixo, onde o vetor $x_{1}$ contém os $\mathrm{GDL}$ que serão preservados no sistema reduzido e $x_{2}$ contém os $\mathrm{GDL}$ que serão eliminadas do sistema completo.

$$
\left\{\begin{array}{c}
\dot{x_{1}} \\
\dot{x_{2}} \\
\hline y
\end{array}\right\}=\left[\begin{array}{cc|c}
A_{11} & A_{12} & B_{1} \\
A_{21} & A_{22} & B_{2} \\
\hline C_{1} & C_{2} & D
\end{array}\right]\left\{\begin{array}{c}
x_{1} \\
x_{2} \\
\hline u
\end{array}\right\}
$$

O método de eliminação determina como serão calculadas as matrizes $A_{r}, B_{r}, C_{r}$ e $D_{r}$ da equação 3 a partir do sistema rearranjado descrito na equação 5 Estes métodos de eliminação serão descritos no capítulo 7 .

Após o cálculo das matrizes que descrevem o sistema reduzido, fica a critério do analista realizar correções para que alguns requisitos sobre o comportamento do mesmo sejam atendidos. Esta etapa é opcional, porém é desejável caso a base e o método de eliminação escolhidos não satisfaçam completamente os requisitos especificados para o modelo de ordem reduzida.

Alguns tipos de análise são comuns ao se realizar o estudo do comportamento de sistemas dinâmicos. Cada abordagem tem objetivos distintos para avaliação de comportamentos específicos do sistema, como por exemplo:

- Análise de estabilidade

- Análise de controlabilidade e observabilidade

- Análise da resposta em estado estável

- Análise da resposta transiente

- Análise modal

De acordo com a aplicação que será dada ao sistema reduzido, o analista deve eleger quais são os requisitos que ele deve satisfazer, objetivando reproduzir características desejadas do sistema completo. De acordo com a metodologia escolhida para redução, pode-se prever se parâmetros como estabilidade, controlabilidade e obervabilidade são preservados. Da mesma maneira, pode-se especificar comportamentos que devem ser preservados no modelo reduzido, 
como características da resposta transitente (tempo de pico, tempo de acomodação, sobresinal máximo), função de transferência em frequências específicas, frequências naturais dos modos de vibrar, etc.

\subsection{ESTABILIDADE}

Ao realizar a redução da ordem de um sistema, é esperado que o sistema reduzido preserve as características principais da resposta dinâmica do sistema completo. Caso o sistema completo seja estável, sua resposta dinâmica não será bem aproximada por um sistema instável. Portanto, espera-se que a estabilidade do sistema seja preservada após a redução.

Existem muitos critérios que se propõem a determinar a estabilidade de um sistema. Neste trabalho, serão adotados dois dos métodos mais utilizados para determinar a estabilidade de sistemas dinâmicos lineares invariantes no tempo, os quais são:

- Sistemas estáveis segundo Lyapunov: O critério de estabilidade de Lyapunov determina que um sistema pode ser considerado estável se possui todos seus autovalores com parte real não positiva.

- Sistemas dinâmicos assintoticamente estáveis: Para um sistema ser considerado assintoticamente estável, deve possuir todos seus autovalores com parte real negativa.

Descrições mais detalhadas sobre a estabilidade de sistemas podem ser encontradas em (OGATA, 2001), (SASTRY, 2013), (ROBINSON, 1995) e (NAYFEH; BALACHANDRAN, 2008).

Um ponto importante a destacar é que a estabilidade de um sistema linear invariante no tempo não depende da base em que está descrito.

\subsection{CONTROLABILIDADE E OBSERVABILIDADE}

Dado um sistema dinâmico linear, contínuo, invariante no tempo ( $\mathrm{LCl}$ ) e de dimensão finita, o conceito de alcançabilidade e controlabilidade são equivalentes. Portanto neste trabalho, o termo utilizado para esta propriedade será controlabilidade.

De acordo com (ZHOU et al., 1996), um sistema dinâmico descrito como no sistema de equações 1 (ou o par $(\mathrm{A}, \mathrm{B})$ ) é dito controlavel se, para um dado estado inicial $x(0)=x_{0}$, $t_{1}>0$ e um estado final $x_{1}$, existe uma função de entrada $u(t)$ de modo que a solução do sistema 1 satisfaça $x\left(t_{1}\right)=x_{1}$. Caso contrário, o sistema é dito não controlável.

Da mesma maneira, um sistema dinâmico descrito como na equação 1 (ou o par $(A, C)$ ) é dito observável se, para qualquer $t_{1}>0$, o estado inicial $x(0)=x_{0}$ pode ser determinado 
a partir das funções entrada $u(t)$ e saída $y(t)$ no intervalo $\left[0, t_{1}\right]$. Caso contrário, é dito não observável.

Para o sistema de equações 1 define-se os gramianos finitos de controlabilidade e observabilidade $L_{c}$ e $L_{o}$ como respectivamente

$$
\begin{aligned}
& L_{c}(t)=\int_{0}^{t} e^{\tau A} B B^{T} e^{\tau A^{T}} d \tau \\
& L_{o}(t)=\int_{0}^{t} e^{\tau A^{T}} C^{T} C e^{\tau A} d \tau
\end{aligned}
$$

Os gramianos também podem ser calculados resolvendo as chamadas equações matriciais de Lyapunov:

$$
\begin{aligned}
& \dot{L}_{c}=A L_{c}+L_{c} A^{T}+B B^{T} \\
& \dot{L}_{o}=A^{T} L_{o}+L_{o} A+C^{T} C
\end{aligned}
$$

Define-se também os gramianos infinitos de controlabilidade e observabilidade como:

$$
\begin{aligned}
& L_{c}=\int_{0}^{\infty} e^{\tau A} B B^{T} e^{\tau A^{T}} d \tau \\
& L_{o}=\int_{0}^{\infty} e^{\tau A^{T}} C^{T} C e^{\tau A} d \tau
\end{aligned}
$$

Para cálculo dos gramianos infinitos, acima descritos, deve ser encontrada a solução estacionária das equações matriciais de Lyapunov. A solução é única se $A$ for estável segundo o critério de Lyapunov e pode ser calculada assumindo que $\dot{L}_{c}=\dot{L}_{o}=0$ :

$$
\begin{aligned}
& A L_{c}+L_{c} A^{T}+B B^{T}=0 \\
& A^{T} L_{o}+L_{o} A+C^{T} C=0
\end{aligned}
$$

Define-se o gramiano infinito cruzado $L_{x}$ como:

$$
L_{x}=\int_{0}^{\infty} e^{\tau A} B C e^{\tau A} d \tau
$$

ou em função do produto dos gramianos de controlabilidade e observabilidade

$$
L_{x}^{2}=L_{c} L_{o}
$$

O gramiano infinito cruzado também pode ser calculado através da solução da chamada equação de Sylvester

$$
A L_{x}+L_{x} A+B C=0
$$


A partir dos conceitos apresentados acima, são definidas as normas Hankel do sistema como a raiz dos autovalores do quadrado do gramiano cruzado:

$$
\gamma_{i}=\sqrt{\lambda_{i}\left(L_{c} L_{o}\right)}=\sqrt{\lambda_{i}\left(L_{x}^{2}\right)}
$$

Uma maneira de se calcular matrizes de controlabilidade $M_{c}$ e observabilidade $M_{o}$ de um sistema LCl pode ser encontrada em (ZHOU; DOYLE, 1998):

$$
\begin{array}{r}
M_{c}=\left[B|A B| A^{2} B|\ldots| A^{n-1} B\right] \\
M_{o}=\left[\begin{array}{c}
C \\
C A \\
C A^{2} \\
\vdots \\
C A^{n-1}
\end{array}\right]
\end{array}
$$

onde $n$ é a dimensão de $A$.

Para sistemas $\mathrm{LCl}$, a condição necessária e suficiente para que o sistema seja controlável é uma das seguintes (ZHOU et al., 1996):

- A matriz de controlabilidade tem posto completo igual a $n$ com $A \in \mathbb{R}^{n x n}$

- O gramiano de controlabilidade $L_{c}(t)$ é positivo definido para qualquer $t>0$

- Nenhum autovetor esquerdo de $A$ está no núcleo esquerdo de $B$. Ou seja, $v^{T} A=\lambda v^{T}$ e $v^{T} B \neq 0$

Analogamente, a condição necessária e suficiente para que o sistema seja observável é uma das seguintes (ZHOU et al., 1996):

- A matriz de observabilidade tem posto completo igual a $n$ com $A \in \mathbb{R}^{n x n}$

- O gramiano de observabilidade $L_{o}(t)$ é positivo definido para qualquer $t>0$

- Nenhum autovetor direito de $A$ está no núcleo direito de $C$. Ou seja, $A v=\lambda v$ e $C v \neq 0$

O gramiano de controlabilidade também está relacionado com a energia necessária para alcançar um estado $x$ a partir de um estado inicial $x_{0}=0$. A função $u_{\min }(t)$ corresponde à entrada necessária para alcançar um estado $x$ no tempo $t_{f}$ de maneira que a energia mecânica gasta seja mínima. 
O quadrado da norma $\mathcal{L}_{2}$ de $u_{\text {min }}$ é

$$
\begin{aligned}
& \left\|u_{\text {min }}\left(t_{f}\right)\right\|_{\mathcal{L}_{2}}^{2}=\left\langle u_{\text {min }}\left(t_{f}\right), u_{\text {min }}\left(t_{f}\right)\right\rangle=\int_{0}^{t_{f}} u_{\text {min }}^{*}(\tau) u_{\text {min }}(\tau) d \tau \\
& \left\|u_{\text {min }}\left(t_{f}\right)\right\|_{\mathcal{L}_{2}}^{2}=x^{*} L_{c}^{-1}\left(t_{f}\right) x
\end{aligned}
$$

Analogamente, a energia da função de saída $y$ no instante de tempo $t_{f}$ observada a partir de uma condição inicial $x_{0} \neq 0$ pode ser calculada pelo quadrado da norma $\mathcal{L}_{2}$ de $y(t)$

$$
\left\|y\left(t_{f}\right)\right\|_{\mathcal{L}_{2}}^{2}=\left\langle y\left(t_{f}\right), y\left(t_{f}\right)\right\rangle=x^{*} L_{o}\left(t_{f}\right) x
$$

Um ponto importante a destacar é que a controlabilidade e observabilidade de um sistema linear invariante no tempo não dependem da base em que está descrito.

\subsection{RESPOSTA EM ESTADO ESTÁVEL}

A análise de resposta em estado estável para entrada senoidal de amplitude unitária pode ser realizada através da chamada função de transferência do sistema. Dado um sistema como na equação 1] sua função de transferência é

$$
G(s)=C(s I-A)^{-1} B+D
$$

A resposta em estado estável do erro pode ser calculada a partir da equação 4 .

$$
G_{e}=C_{e}\left(s I-A_{e}\right)^{-1} B_{e}+D_{e}
$$

onde

$$
\begin{aligned}
A_{e} & =\left[\begin{array}{ll}
A & 0 \\
0 & A_{r}
\end{array}\right] \\
C_{e} & =\left[\begin{array}{c}
C-C_{r}
\end{array}\right] \\
B_{e} & =\left[\begin{array}{c}
B \\
B_{r}
\end{array}\right] \\
D_{e} & =D-D_{r}
\end{aligned}
$$

Dois valores de resposta em frequência merecem especial destaque: $\lim _{s \rightarrow \infty} G(s)$ e $\lim _{s \rightarrow 0} G(0)$, que neste trabalho serão chamadas respectivamente de resposta em altas frequências e resposta estática do sistema. 


\subsubsection{Resposta em altas frequências}

A chamada resposta em altas frequências é a amplitude do sinal de saída da função de transferência para uma frequência de entrada que tende a infinito. Sendo assim, será usado o abuso de notação $\lim _{s \rightarrow \infty} G(s)=G(\infty)$.

Dado um sistema como no sistema de equações 2 sua resposta em altas frequências é

$$
\lim _{s \rightarrow \infty} G(s)=G(\infty)=D
$$

Analogamente, a resposta em altas frequências do erro do sistema de ordem reduzida é

$$
\lim _{s \rightarrow \infty} G_{e}(s)=G_{e}(\infty)=D_{e}
$$

\subsubsection{Resposta estática}

Existe um caso particular para a resposta em estado estável para uma frequência igual a zero $(G(0))$, esta condição acontece quando o sitema se encontra em equilíbrio para uma entrada de valor constante ao longo do tempo $u(t)=u_{e q}$. Neste trabalho, esta condição será chamada de estática ou de equilíbrio.

Dado um sistema estável, na forma do sistema de equações 11 submetido à uma entrada estática $u_{e q}$ temos a condição de equilíbrio dada por:

$$
\left\{\begin{array}{l}
A x_{e q}+B u_{e q}=0 \\
y_{e q}=C x_{e q}+D u_{e q}
\end{array}\right.
$$

onde $x_{e q}$ e $y_{e q}$ são os vetores de estado e de resposta do sistema na condição de equilíbrio respectivamente.

Substituindo 23 em 4 temos o erro $e_{e q}$ da resposta estática do sistema reduzido:

$$
\left[\begin{array}{cc|c}
A & 0 & 0 \\
0 & A_{r} & 0 \\
\hline C & -C_{r} & -I
\end{array}\right]\left\{\begin{array}{c}
x_{e q} \\
x_{r e q} \\
\hline e_{e q}
\end{array}\right\}=\left\{\begin{array}{c}
-B \\
-B_{r} \\
\hline-D+D_{r}
\end{array}\right\} u_{e q}
$$

onde $x_{r e q}, y_{r e q}$ e $e_{e q}$ são respectivamente os vetores de estado, resposta e de erro da resposta estática do sistema de ordem reduzida.

$$
e_{e q}=y_{e q}-y_{r e q}
$$




\subsection{CONSIDERAÇÕES SOBRE CUSTO COMPUTACIONAL}

Um outro tema a ser considerado no processo de redução de modelos é a dificuldade para se realizar determinadas operações utilizando algoritmos executados por um computador. Este tópico é importante pois em aplicações onde a ordem dos modelos envolvidos é grande, a quantidade de memória e o tempo necessários para executar estas operações podem ser insatisfatórios de acordo com as expectativas envolvidas no projeto.

Además, o custo computacional para executar determinada operação numérica depende dos algoritmos usados na sua implementação (ARORA; BARAK, 2009).

Neste trabalho, o custo computacional de uma operação será abordado de maneira qualitativa sem que sejam aplicadas técnicas para mensurar o mesmo. Portanto para que seja possível uma comparação qualitativa entre dois procedimentos computacionais, ambos devem ser executados pelos mesmos algoritmos e apenas diferir quanto à ordem das matrizes e vetores envolvidos.

Como exemplo de tal comparação qualitativa, sabe-se que o custo computacional para calcular a solução de um sistema linear do tipo $A x=b$ depende da ordem da matriz $A$. Portanto o custo computacional para resolver um sistema de ordem $n_{1}$ é menor que para um sistema de ordem $n_{2}$ se $n_{1} \leq n_{2}$. 


\section{MUDANÇAS DE BASE}

No processo de redução de um modelo, a base em que ele está definido tem forte influência na escolha dos graus de liberdade que serão reduzidos e na representatividade que o modelo reduzido terá em relação ao completo (OBINATA; ANDERSON, 2001). Por isso, ao realizar a redução de um sistema, pode-se fazer uma mudança de base que seja conveniente para que o modelo reduzido seja mais fiel ao completo.

\subsection{BASE DE FORMAS MODAIS (BFM)}

No item 5.1, será feita uma breve descrição da mudança de base de um sistema para uma base de formas modais (BFM). Para tal, o autor se baseou nas referências (GAWRONSKI, 1998), (SOARES, 1998), (FU; HE, 2001) e (MEIROVITCH, 1980), onde pode-se encontrar descrições mais detalhadas sobre o assunto.

Dado um sistema LCl com $n_{0}$ coordenadas generalizadas, do tipo

$$
M \ddot{q}+C_{a} \dot{q}+K q=F u
$$

Sendo $q \in \mathbb{R}^{n_{0}}$ é o vetor de coordenadas generalizadas do sistema e as matrizes $M, C_{a}$ e $K$ são quadradas de ordem $n_{0}$. Também assume-se que $M$ é positiva definida, portanto existe a matriz inversa $M^{-1}$.

Considera-se um caso genérico onde o amortecimento do sistema em questão é dito não proporcional, ou seja, a matriz de amortecimento $C_{a}$ não pode ser escrita como uma combinação linear das matrizes $M$ e $K$.

Deseja-se projetar o sistema em uma base de autovetores, que posteriormente será interpretada como uma sobreposição de modos de vibração do sistema. Definem-se como modos de vibração síncronos de um sistema os padrões de movimento oscilatório em que coordenadas generalizadas possuem relações de proporcionalidade constantes.

Considerando o sistema não excitado na forma

$$
M \ddot{q}+C_{a} \dot{q}+K q=0
$$

Através da mudança de base $x=\left(q^{T}, \dot{q}^{T}\right)^{T}$, o sistema de segunda ordem com $n_{0}$ equações diferenciais ordinárias (EDO) pode ser escrito como um sistema de primeira ordem com $n=$ $2 n_{0}$ EDO. Desta maneira, a equação 27 pode ser escrita na forma:

$$
\left\{\begin{array}{c}
\dot{q} \\
\ddot{q}
\end{array}\right\}=\left[\begin{array}{cc}
0 & I \\
-M^{-1} K & -M^{-1} C_{a}
\end{array}\right] \cdot\left\{\begin{array}{l}
q \\
\dot{q}
\end{array}\right\}
$$


Onde $x \in \mathbb{R}^{2 n_{0}}$ é um vetor que contém deslocamentos e velocidades generalizadas do sistema. Desta forma, pode-se escrever sinteticamente:

$$
\dot{x}=A x
$$

Deseja-se agora encontrar uma base a fim de realizar a decomposição do sistema em modos de vibrar, desta maneira a solução da equação 29 é da forma

$$
x(t)=e^{s t} z
$$

onde s é um escalar e $z \in \mathbb{R}^{n}$ que caracteriza as formas modais de vibração do sistema. Substituindo-se 30 em 29 , obtém-se

$$
A z_{k}=\lambda_{k} z_{k} \quad k=1,2, \ldots, n_{0}
$$

A partir da equação 31 , pode-se concluir que $z_{k}$ é o k-ésimo autovetor direito de $\mathrm{A}$ e $\lambda_{k}$ é seu autovalor associado. Neste trabalho, serão considerados apenas casos onde os autovalores do sistema são distintos, ou seja, não existe $\lambda_{i}=\lambda_{j}$ para $i \neq j$.

Pode-se escrever cada autovetor em duas parcelas $z_{a}, z_{b} \in \mathbb{R}^{n_{0}}$

$$
z=\left(z_{a}^{T}, z_{b}^{T}\right)^{T}
$$

Substituindo a equação 32 em 31 encontra-se o seguinte sistema:

$$
\begin{aligned}
z_{b} & =s z_{a} \\
-M^{-1} K z_{a}-M^{-1} D z_{b} & =s z_{b}
\end{aligned}
$$

O sistema de equações acima tem soluções não triviais do tipo

$$
\lambda_{k}=\alpha_{k} \pm i \beta_{k}
$$

que são encontradas ao se resolver a equação 35

$$
\operatorname{det}\left(M s^{2}+D s+K\right)=0
$$

Observa-se que a equação 35 possui $n$ soluções não triviais $\lambda_{k}$ que também são autovalores da matriz $A$ na equação 31 . Pode-se provar (SOARES, 1998) que estas soluções podem ser reais e/ou pares pares complexos conjugados. Consequentemente, seus autovetores correspondentes são respectivamente reais e complexos conjugados. Neste último caso, pode-se encontrar: 


$$
\begin{aligned}
& A z_{k}=\lambda_{k} z_{k} \\
& A \bar{z}_{k}=\bar{\lambda}_{k} \bar{z}_{k}
\end{aligned}
$$

onde

$$
z_{k}=u_{k}+i v_{k} \quad \text { e } \quad \bar{z}_{k}=u_{k}-i v_{k}
$$

Sem que exista perda de generalização, a partir deste ponto as equações serão apresentadas considerando que todos os autovalores do sistema são complexos conjugados. O caso particular para autovalores reais é deduzido de maneira análoga.

Seja uma matriz $\Phi$ de ordem $n$, cujas colunas são as componentes reais e complexas de todos os autovetores direitos de $A$

$$
\Phi=\left[\begin{array}{llllllll}
u_{1} & v_{1} & \ldots & u_{k} & v_{k} & \ldots & u_{n_{0}} & v_{n_{0}}
\end{array}\right]
$$

Onde os vetores $u_{k}$ e $v_{k}$ são chamados de formas modais que compõem um modo de vibrar do sistema.

Pode-se demonstrar (HIRSCH; DEVANEY; SMALE, 1974) que a matriz $\Phi$ é inversível e que é uma matriz de mudança de base do tipo

$$
x=\Phi w
$$

Aplicando-se a transformação descrita da equação 39 ao sistema da equação 2 obtém-se:

$$
\left\{\begin{array}{c}
\Phi \dot{w}=A \Phi w+B u \\
y=C \Phi w+D u
\end{array}\right.
$$

onde

$$
B=M^{-1} F
$$

Assim, pode-se definir o sistema $[\tilde{A}, \tilde{B}, \tilde{C}, \tilde{D}]$ na base modal, com:

$$
\begin{gathered}
\widetilde{A}=\Phi^{-1} A \Phi \\
\widetilde{B}=\Phi^{-1} B \\
\widetilde{C}=C \Phi \\
\widetilde{D}=D
\end{gathered}
$$


e consequentemente

$$
\left\{\begin{array}{c}
\dot{w} \\
\hline y
\end{array}\right\}=\left[\begin{array}{c|c}
\Phi^{-1} A \Phi & \Phi^{-1} B \\
\hline C \Phi & D
\end{array}\right] \cdot\left\{\begin{array}{c}
w \\
\hline u
\end{array}\right\}
$$

Sendo assim, a matriz $\widetilde{A}$, de ordem $n=2 n_{0}$, é composta por blocos $2 \times 2$ correspondentes aos pares de autovalores complexos de $A$ (e blocos $1 \times 1$ para o caso de autovalores reais).

Desta maneira, as matrizes do sistema na base modal podem ser escritas como:

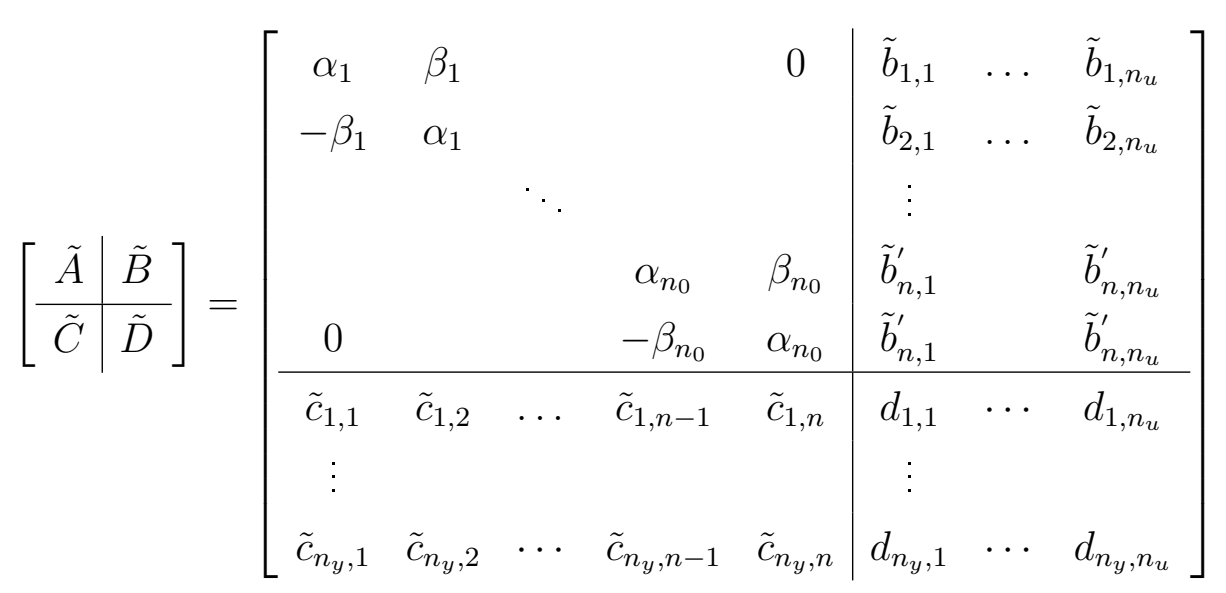

A equação 43 pode ser desacoplada em subsistemas de ordem 2 (para o caso de autovalores complexos), como mostrados na equação 45. Cada subsistema descreve a dinâmica de um modo de vibração e é formado pelas matrizes $\left[A_{k}, B_{k}, C_{k}, D_{k}\right]$. A resposta $y_{k}$ é chamada de participação modal do modo $k$ na resposta do sistema.

$$
\left\{\begin{array}{c}
\dot{w}_{2 k-1} \\
\dot{w}_{2 k} \\
\hline y_{k}
\end{array}\right\}=\left[\begin{array}{cc|ccc}
\alpha_{k} & \beta_{k} & \tilde{b}_{2 k-1,1} & \cdots & \tilde{b}_{2 k-1, n_{u}} \\
-\beta_{k} & \alpha_{k} & \tilde{b}_{2 k, 1} & \cdots & \tilde{b}_{2 k, n_{u}} \\
\hline \tilde{c}_{1,2 k-1} & \tilde{c}_{1,2 k} & 0 & \cdots & 0 \\
\vdots & & \vdots & & \\
\tilde{c}_{n_{y}, 2 k-1} & \tilde{c}_{n_{y}, 2 k} & 0 & \cdots & 0
\end{array}\right]\left\{\begin{array}{c}
w_{2 k-1} \\
w_{2 k} \\
\hline u
\end{array}\right\}
$$

sendo que $k=1,2, \ldots, n_{0}$.

Vale lembrar que o vetor $y(t)$ é resultado da soma das participações modais $y_{k}(t)$ com o termo $D u$ :

$$
y(t)=\sum_{k=1}^{n_{0}} y_{k}(t)+D u(t)
$$




\subsubsection{Vibração livre}

Considerando o problema de vibração livre, com $u=0$, dada uma condição inicial $\omega_{0}=$ $\omega(t=0)$ encontra-se uma série de soluções acopladas duas a duas na forma

$$
\left\{\begin{array}{c}
\dot{w}_{2 k-1} \\
\dot{w}_{2 k}
\end{array}\right\}=\left[\begin{array}{cc}
\alpha_{k} & \beta_{k} \\
-\beta_{k} & \alpha_{k}
\end{array}\right]\left\{\begin{array}{c}
w_{2 k-1} \\
w_{2 k}
\end{array}\right\}
$$

com $k=1,2, \ldots, n_{0}$, cujas soluções para o problema de condições iniciais são

$$
\begin{aligned}
& w_{2 k-1}(t)=e^{\alpha_{k} t}\left[a_{k} \cos \left(\beta_{k} t\right)+b_{k} \operatorname{sen}\left(\beta_{k} t\right)\right] \\
& w_{2 k}(t)=e^{\alpha_{k} t}\left[-a_{k} \operatorname{sen}\left(\beta_{k} t\right)+b_{k} \cos \left(\beta_{k} t\right)\right]
\end{aligned}
$$

sendo que $a_{k}$ e $b_{k}$ são constantes que dependem das condições iniciais do problema.

$$
\begin{aligned}
& a_{k}=w_{2 k-1}(0) \\
& b_{k}=w_{2 k}(0)
\end{aligned}
$$

As condições iniciais na base de autovetores podem ser calculadas a partir da base de coordenadas generalizadas, através da relação

$$
w(0)=\Phi^{-1} x(0)
$$

É possível perceber que as formas modais (associadas a autovalores complexos não repetidos) de um sistema com amortecimento não proporcional são acopladas duas a duas de maneira que existe uma diferença de fase entre $w_{2 k-1}(t)$ e $w_{2 k}(t)$. Ou seja, dado $a_{k} \neq 0$, no instante que $w_{2 k-1}(t)$ se anular, $w_{2 k}(t)$ será não nulo. Analogamente quando $w_{2 k}(t)$ se anular, $w_{2 k-1}(t)$ será diferente de zero.

Este comportamento caracterizado pela alternância entre as formas modais $u_{k}$ e $v_{k}$ forma uma onda não-estacionária, fazendo com que os modos de vibração sejam chamados de assíncronos.

Outra característica importante deste tipo de sistema dinâmico é que $u_{k}$ e $v_{k}$ definem um subespaço bidimensional $S_{k}$ no espaço de fase. Este subespaço é dito invariante ao fluxo, ou seja, se o sistema tiver condições iniciais que pertençam a $S_{k}$, sua solução permanecerá em $S_{k}$ (SOARES, 1998).

É possível mostrar, por absurdo, que $u_{k}$ e $v_{k}$ são sempre linearmente independentes, garantindo portanto a existência do subespaço bidimensional $S_{k}$.

Prova: Dado um autovalor $\lambda_{k}$ complexo, seu autovetor correspondente $z_{k}$ também é composto por uma parte real e outra complexa. Caso a parte real e imaginária de $z_{k}$ sejam linearmente dependentes, $z_{k}$ pode ser escrito na forma $z_{k}=(1+i \gamma) \operatorname{Re}\left(z_{k}\right)$ e substituído 
na equação 31. Após simplificações, conclui-se que o vetor $\operatorname{Re}\left(z_{k}\right)$ satisfaz da equação 31 . contradizendo a hipótese inicial de que $z_{k}$ teria parte complexa não nula.

Aplicando-se uma condição inicial ao sistema, que seja combinação linear de $u_{k}$ e $v_{k}$, que faz com que a solução da equação 43 seja

$$
x(t)=w_{2 k-1}(t) u_{k}+w_{2 k}(t) v_{k}
$$

A figura 2 mostra um exemplo de movimento em onda não estacionária, causado por um modo assíncrono. Para construção da figura, foi usado o primeiro modo de vibrar do estudo de caso descrito no capítulo 10 com condições iniciais tais que $a_{1}=0,2$ e $b_{1}=0,2$.

Figura 2 - Exemplo de modo assíncrono.

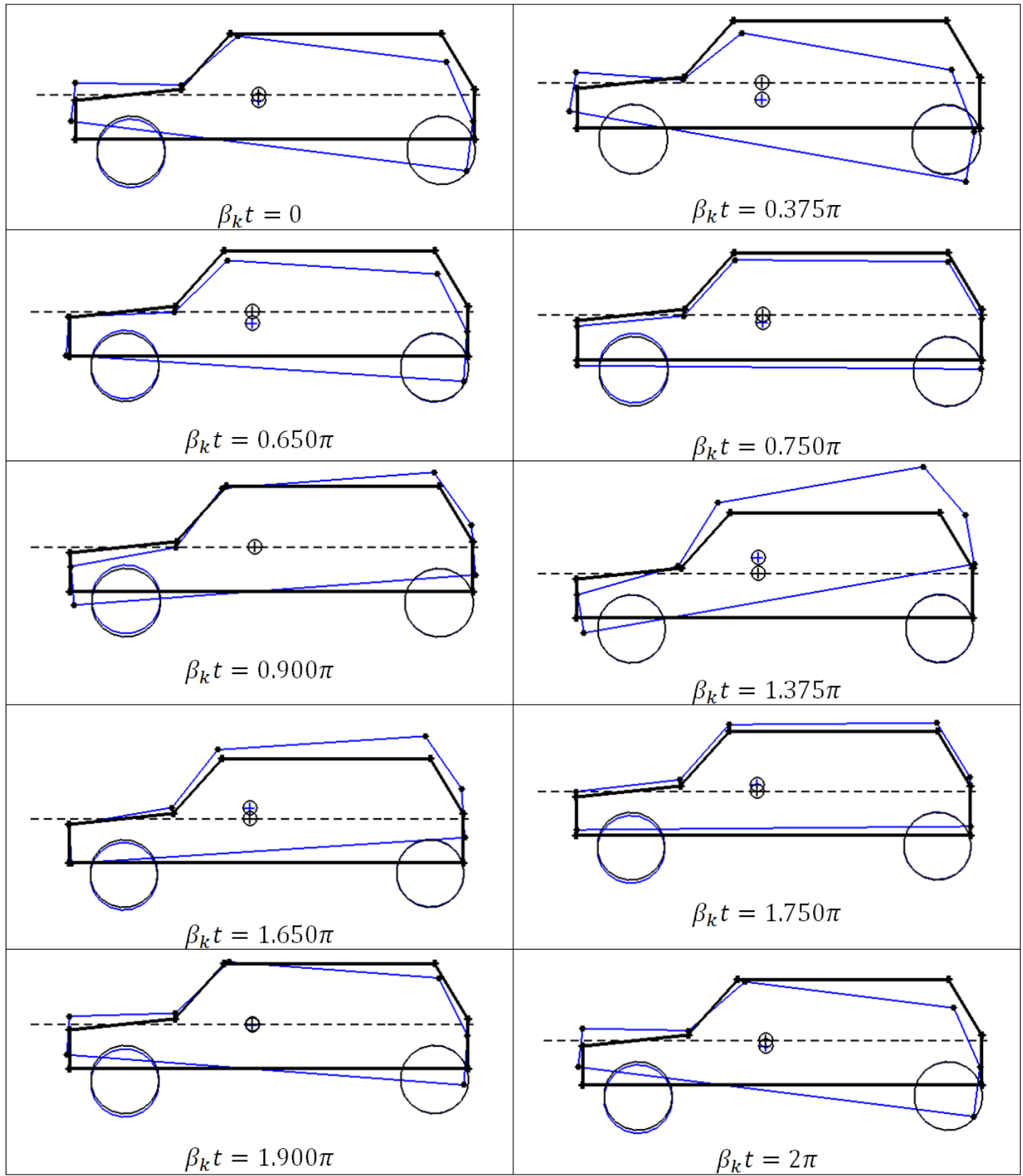


A partir da figura 2, observa-se duas situações onde o deslocamento vertical do centro de massa do chassi é nulo $\left(\beta_{k} t=0,9 \pi\right.$ e $\left.\beta_{k} t=1,9 \pi\right)$. O ângulo de arfagem nestes dois momentos apresenta sinais opostos. Esta característica típica de movimentos de onda não estacionária. O mesmo comportamento pode ser observado nas situações $\beta_{k} t=0,75 \pi$ e $\beta_{k} t=1,75 \pi$, onde o ângulo de arfagem é nulo e o deslocamento vertical tem sinais opostos.

De maneira mais abrangente, a solução geral da equação 43 para qualquer condição inicial é:

$$
x(t)=w_{1}(t) u_{1}+w_{2}(t) v_{1}+\cdots+w_{2 n_{0}-1}(t) u_{n_{0}}+w_{2 n_{0}}(t) v_{n_{0}}
$$

Cabe lembrar que existem grandezas que auxiliam o estudo do sistema na base modal e são definidas para cada modo de vibrar, como: frequência natural não amortecida $\omega_{n k}$, frequência natural amortecida $\omega_{d k}$ e fator de amortecimento $\zeta_{k}$. Onde o índice $k$ indica o k-ésimo modo.

$$
\begin{aligned}
& \omega_{n k}=\sqrt{\alpha_{k}^{2}+\beta_{k}^{2}} \\
& \omega_{d k}=\beta_{k} \\
& \zeta_{k}=\frac{-\alpha_{k}}{\sqrt{\alpha_{k}^{2}+\beta_{k}^{2}}}
\end{aligned}
$$

\subsubsection{Resposta em frequência}

Partindo para uma abordagem no domínio da frequência, pode-se escrever a função de transferência do sistema como:

$$
G(s)=\widetilde{D}+\widetilde{C}(s I-\widetilde{A})^{-1} \widetilde{B}
$$

Para encontrar uma expressão da função de transferência do sistema na base modal, pode-se escrever a matriz diagonal por blocos:

$$
(s I-\widetilde{A})^{-1}=\left[\begin{array}{ccc}
\Lambda_{1}^{-1}(s) & & 0 \\
& \ddots & \\
0 & & \Lambda_{n_{0}}^{-1}(s)
\end{array}\right]
$$

Onde:

$$
\Lambda_{k}(s)=\left[\begin{array}{cc}
s-\alpha_{k} & \beta_{k} \\
-\beta_{k} & s-\alpha_{k}
\end{array}\right]
$$

e

$$
\Lambda_{k}^{-1}(s)=\frac{1}{\left(s-\alpha_{k}\right)^{2}+\beta_{k}^{2}}\left[\begin{array}{cc}
s-\alpha_{k} & \beta_{k} \\
-\beta_{k} & s-\alpha_{k}
\end{array}\right]
$$


A função de transferência $G(s)$ do sistema na base modal é:

$$
G(s)=D+\left[\begin{array}{ccc}
\tilde{c}_{1,1} & \ldots & \tilde{c}_{1, n} \\
\vdots & & \\
\tilde{c}_{n_{y}, 1} & \ldots & \tilde{c}_{n_{y}, n}
\end{array}\right] \cdot\left[\begin{array}{ccc}
\Lambda_{1}^{-1}(s) & & 0 \\
\vdots & \ddots & \\
0 & & \Lambda_{n_{0}}^{-1}(s)
\end{array}\right] \cdot\left[\begin{array}{ccc}
\tilde{b}_{1,1} & \ldots & \tilde{b}_{1, n_{u}} \\
\vdots & & \\
\tilde{b}_{n, 1} & \ldots & \tilde{b}_{n, n_{u}}
\end{array}\right]
$$

Pode-se escrever a função de transferência $G_{k}(s)$ para um o modo $k$, onde $G_{k}(s) \in \mathbb{C}^{n_{y} \times n_{u}}$

$$
G_{k}(s)=\left[\begin{array}{cc}
\tilde{c}_{1,2 k-1} & \tilde{c}_{1,2 k} \\
\vdots & \\
\tilde{c}_{n_{y}, 2 k-1} & \tilde{c}_{n_{y}, 2 k}
\end{array}\right] \cdot\left[\Lambda_{k}^{-1}(s)\right] \cdot\left[\begin{array}{ccc}
\tilde{b}_{2 k-1,1} & \ldots & \tilde{b}_{2 k-1, n_{u}} \\
\tilde{b}_{2 k, 1} & \ldots & \tilde{b}_{2 k, n_{u}}
\end{array}\right]
$$

A função de transferência do sistema é portanto a soma das funções modais acrescidas da parcela $D$

$$
G(s)=D+\sum_{k=1}^{n_{0}} G_{k}(s)
$$

A partir da equação 60 pode-se obervar que a função de transferência $G(s)$ é a somatória das funções de transferência modais $G_{k}(s)$. A função de transferência modal $G_{k}(s)$ tem maior influência na resposta do sistema quando a frequência de excitação está próxima à sua frequência amortecida $\omega_{d k}$. A figura 3 mostra um exemplo de uma função de transferência do sistema e as componentes $G_{k}(s)$ dos 4 primeiros modos.

Figura 3 - Exemplo da participação das funções de transferência dos modos na resposta do sistema.

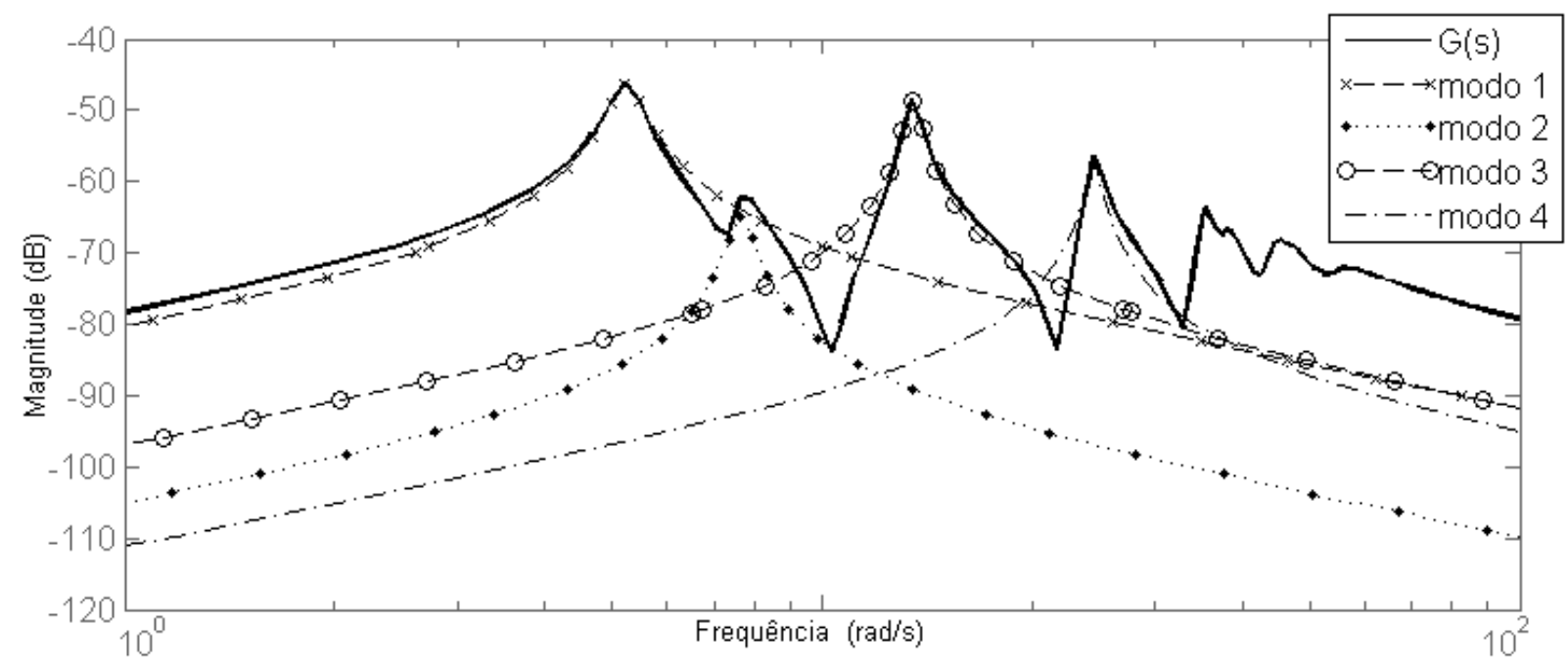

Para o caso particular de sistemas não amortecidos ou com amortecimento proporcional, é possível provar que seus autovalores terão parte real nula (MEIROVITCH, 1980). Desta 
maneira, a matriz $A$ será diagonal

$$
\widetilde{A}=\Phi^{-1} A \Phi=\left[\begin{array}{ccc}
\lambda_{1} & & 0 \\
& \ddots & \\
0 & & \lambda_{n}
\end{array}\right]
$$

Desta maneira, pode-se escrever o valor da função de transferência $G(s)$ do sistema na forma:

$$
G(s)=\widetilde{C}\left[\begin{array}{ccc}
\frac{1}{s-\lambda_{1}} & & 0 \\
& \ddots & \\
0 & & \frac{1}{s-\lambda_{n}}
\end{array}\right] \widetilde{B}
$$

ou

$$
G(s)=\sum_{k=1}^{n}\left[\begin{array}{ccc}
\frac{\tilde{c}_{1, k} \tilde{b}_{k, 1}}{s-\lambda_{k}} & \ldots & \frac{\tilde{c}_{1, k} \tilde{b}_{k, n_{u}}}{s-\lambda_{k}} \\
\vdots & \ddots & \\
\frac{\tilde{c}_{n_{y}, k} \tilde{b}_{k, 1}}{s-\lambda_{k}} & & \frac{\tilde{c}_{n_{y}, k} \tilde{b}_{k, n_{u}}}{s-\lambda_{k}}
\end{array}\right]
$$

Onde os produtos $\tilde{c}_{1, k} \tilde{b}_{k, 1}$ são comumente chamado resíduos da função de transferência.

\subsection{BASE DE VETORES SINGULARES}

Outra forma importante de mudança de base é através da decomposição em valores singulares (DVS), que projeta o sistema na base de vetores singulares (FU; HE, 2001).

Inicialmente, deseja-se encontrar a solução da equação

$$
A v_{1}=\sigma_{1} u_{1}
$$

que maximiza o valor do escalar $\sigma_{1}$. Em outras palavras, o valor de $\sigma_{1}$ é o máximo ganho que o operador linear $A$ realiza em um vetor unitário $v_{1}$ mesmo que a direção do vetor que é resultado da operação seja modificada. Define-se que $\sigma_{1}$ é chamado de primeiro valor singular de $A$ pois é o máximo valor $\|A x\|_{2}$ para qualquer vetor unitário $x$.

Em (LAY, 2003), é mostrado que o segundo valor singular de $A$ é o máximo ganho sobre todos os vetores unitários ortogonais a $v_{1}$. Portanto, o método diagonaliza uma matriz $A \in \mathbb{C}^{n \times m}, n \leq m$ de forma que

$$
A v_{i}=\sigma_{i} u_{i} \quad i=1,2, \ldots, n
$$


onde $u_{i}$ e $v_{i}$ são vetores unitários que pertencem a $\mathbb{R}^{n}$ e $\mathbb{R}^{m}$ respectivamente e $\sigma_{i} \geq 0$ é um escalar. A figura 4 mostra uma representação gráfica do efeito que o operador linear $A$ realiza sobre um círculo unitário em $\mathbb{R}^{2}$.

Figura 4 - Representação em $\mathbb{R}^{2}$ do operador $A$ diagonalizado pela DVS
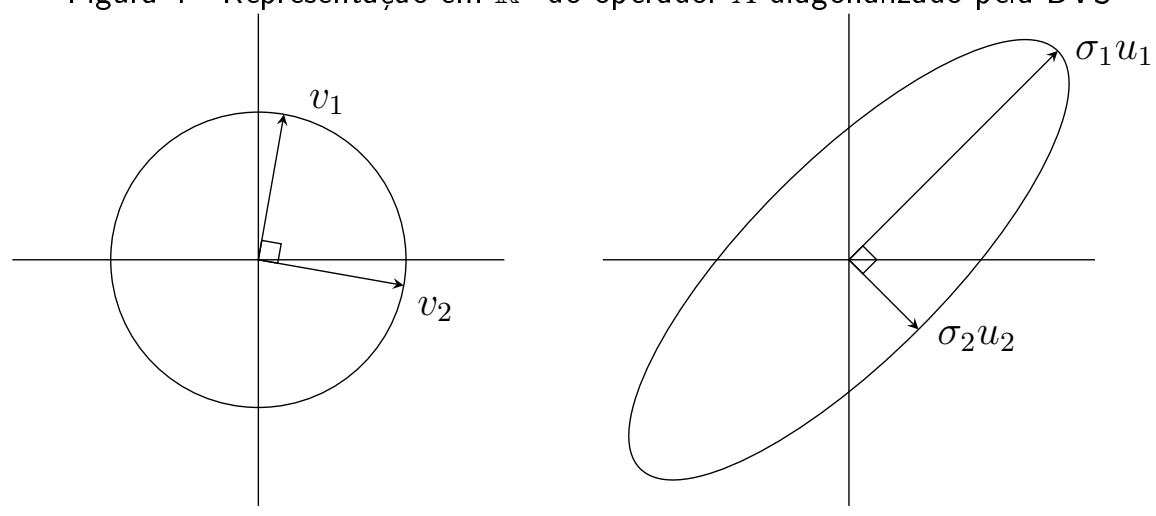

Vale salientar que a condição $n \leq m$ foi dada inicialmente com objetivo de facilitar a exposição do tópico e não restringe as conclusões aqui apresentadas. A condição $n \geq m$ pode ser deduzida de maneira análoga e encontrada em (ANTOULAS, 2005).

A diagonalização da matriz $A$ pela decomposição em valores singulares satisfaz a equação

$$
A V_{v s}=U_{v s} \Sigma_{v s}
$$

onde $\Sigma_{v s}$ é diagonal e

$$
\begin{aligned}
& U_{v s}=\left[u_{1}, u_{2}, \ldots, u_{n}\right] \quad \mathrm{e} \\
& V_{v s}=\left[v_{1}, v_{2}, \ldots, v_{m}\right]
\end{aligned}
$$

As colunas da matriz $U_{v s}$ e $V_{v s}$ são chamadas de vetores singulares à esquerda e à direita da matriz $A$ respectivamente (LAY, 2003).

Como $U_{v s}$ e $V_{v s}$ são matrizes unitárias e ortogonais, $U_{v s}^{*} U_{v s}=I_{n}$ e $V_{v s}^{*} V_{v s}=I_{m}$. Onde $I_{n}$ e $I_{m}$ são matrizes identidade de ordem $n$ e $m$ respectivamente. Desta maneira, pode-se escrever a partir da equação 66

$$
A A^{*}=U_{v s} \Sigma_{v s} V_{v s}^{*} V_{v s} \Sigma_{v s} U_{v s}^{*}=U_{v s} \Sigma_{v s}^{2} U_{v s}^{*}
$$

Portanto, os autovetores e autovalores de $A A^{*}$ formam respectivamente as colunas de $U_{v s}$ e a diagonal de $\Sigma_{v s}^{2}$. Como $A A^{*}$ é positiva semi-definida, seus autovalores são reais não negativos.

$$
\Sigma_{v s}=\operatorname{diag}\left\{\sigma_{1}, \sigma_{2}, \ldots, \sigma_{n}\right\} \quad \text { com } \quad\left\{\sigma_{1} \geq \sigma_{2} \geq \ldots \sigma_{n} \geq 0\right\}
$$

Onde $\sigma_{1}, \sigma_{2}, \ldots, \sigma_{n}$ são chamados de valores singulares da matriz $\mathrm{A}$. 
Define-se também que o maior valor singular $\sigma_{1}$ é a norma quadrática (ou norma $\mathcal{L}_{2}$ ) da matriz $A$, indicada pela notação $\|A\|_{\mathcal{L}_{2}}$. (STRANG, 2006)

$$
\|A\|_{\mathcal{L}_{2}}=\max _{x \neq 0} \frac{\|A x\|_{\mathcal{L}_{2}}^{2}}{\|x\|_{\mathcal{L}_{2}}^{2}}=\sqrt{\lambda_{\max }\left(A A^{*}\right)}
$$

Analogamente, a matriz $V_{v s}$ é formada pelos autovetores de $A^{*} A$ como mostra a equação 68

$$
A^{*} A=V_{v s} \Sigma_{v s} U_{v s}^{*} U_{v s} \Sigma_{v s} V_{v s}^{*}=V_{v s} \Sigma_{v s}^{2} V_{v s}^{*}
$$

Expandindo a equação 66 , encontra-se a seguinte relação:

$$
A=\sum_{i=1}^{n} u_{i} \sigma_{i} v_{i}^{*}
$$

Porém, pode-se encontrar a norma de cada matriz que compõe somatória da equação 69 a partir da relação

$$
\left\|u_{i} v_{i}^{*}\right\|_{\mathcal{L}_{2}}=\lambda_{\max }\left(u_{i} v_{i}^{*} v_{i} u_{i}^{*}\right)=1
$$

Pois os vetores $u_{i}$ e $v_{i}$ são unitários e $\lambda_{\max }\left(u_{i} v_{i}^{*} v_{i} u_{i}^{*}\right)$ é o maior autovalor (em módulo) da matriz.

Portanto:

$$
\left\|\sigma_{1} u_{1} v_{1}^{*}\right\|=\sigma_{1} \geq\left\|\sigma_{2} u_{2} v_{2}^{*}\right\|=\sigma_{2} \geq \cdots \geq\left\|\sigma_{n} u_{n} v_{n}^{*}\right\|=\sigma_{n}
$$

Portanto a matriz $A$ pode ser escrita como uma somatória de matrizes organizadas por ordem decrescente da mais significativa para a menos significativa.

Algumas propriedades sobre a DVS são

- Seja $r$ o posto da matriz $A$ e $r \leq n \leq m$

$$
\begin{aligned}
& -\operatorname{spanCol}(A)=\operatorname{span}\left\{u_{1}, \ldots, u_{r}\right\} \\
& -\operatorname{spanCol}\left(A^{*}\right)=\operatorname{span}\left\{v_{1}, \ldots, v_{r}\right\} \\
& -\operatorname{Nul}\left(A^{*}\right)=\operatorname{span}\left\{u_{r+1}, \ldots, u_{n}\right\} \\
& -\operatorname{Nul}(A)=\operatorname{span}\left\{v_{r+1}, \ldots, v_{m}\right\}
\end{aligned}
$$

Onde $\operatorname{span} \operatorname{Col}(A)$ é o espaço formado pelas colunas linearmente independentes da matriz $A$ e $N u l(A)$ é o núcleo (também chamado de Kernel ou espaço nulo) de $A$. 
Projetando o vetor $x$ da equação 1 na base formada pelas colunas da matriz $V_{v s}$ (também chamada de base de vetores singulares), encontra-se:

$$
V_{v s} w_{v s e}=x
$$

onde o índice vse indica o estado do sistema na base de vetores singulares à esquerda de $A$, encontra-se:

$$
\left\{\begin{array}{l}
\dot{w}_{v s e}=V_{v s}^{*} U_{v s} \Sigma_{v s} w_{v s e}+V_{v s}^{*} B u \\
y=C V_{v s} w_{v s e}+D u
\end{array}\right.
$$

ou

$$
\left\{\begin{array}{c|c}
\dot{w}_{v s e} \\
\hline y
\end{array}\right\}=\left[\begin{array}{c|c}
V_{v s}^{*} A V_{v s} & V_{v s}^{*} B \\
\hline C V_{v s} & D
\end{array}\right] \cdot\left\{\begin{array}{c}
w_{v s e} \\
\hline u
\end{array}\right\}
$$

Pode-se perceber que a diagonalização de uma matriz na base de autovetores é um caso particular da DVS, onde a matriz $A$ é quadrada e os vetores $v_{i}$ e $u_{i}$ na equação 65 são linearmente dependentes.

Uma das vantagens da DVS é o fato de que a diagonalização de uma matriz na forma $A=U_{v s} \Sigma_{v s} V_{v s}^{*}$ é sempre possível, ao contrário da forma $A=\Phi \widetilde{A} \Phi^{-1}$ (STRANG, 2006).

Um exemplo numérico da projeção de um sistema linear em uma base de vetores singulares pode ser encontrada em anexo no item C.1

\subsection{ESPAÇO DE SÉRIES DE POTÊNCIA (BASE DE KRYLOV)}

No item 5.3 será feita uma breve descrição da projeção de um sistema para uma base de Krylov. Para tal, o autor se baseou nas referências (ANTOULAS, 2005), (SCHILDERS; VORST; ROMMES, 2008), (MOHAMMADPOUR; GRIGORIADIS, 2010) e (BENNER; MEHRMANN; SORENSEN, 2005), onde pode-se encontrar descrições mais detalhadas sobre o assunto.

Esta técnica se baseia na projeção do sistema completo em um espaço de dimensão $i$ definido por:

$$
K^{i}(A ; v)=\operatorname{span}\left\{v, A v, A^{2} v, \ldots, A^{i-1} v\right\}
$$

com $A \in \mathbb{R}^{n \times n}$ e $v \in \mathbb{R}^{n}$.

Este conjunto de vetores é conhecido por Espaço de Krylov.

Deseja-se encontrar uma função de transferência reduzida $G_{r}(s)$ que seja uma aproximação de $G(s)$, de maneira que o novo sistema tenha um comportamento dinâmico semelhante ao 
do completo.

$$
G(s)=D+C(s I-A)^{-1} B=D+C A^{-1}\left(s A^{-1}-I\right)^{-1} B
$$

Realizando uma expansão em série de Taylor em torno de $s=s_{0}$ (SALIMBAHRAMI; LOHMANN, 2002), encontra-se:

$$
(s I-A)^{-1}=\lim _{k \rightarrow \infty}\left(A-I s_{0}\right)+\left(A-I s_{0}\right)^{2} s+\cdots+\left(A-I s_{0}\right)^{k} s^{k-1}
$$

Substituindo 75 em 74 obtém-se:

$$
G(s)=D+\lim _{k \rightarrow \infty}\left[C\left(A-I s_{0}\right) B+C\left(A-I s_{0}\right)^{2} B s+\cdots+C\left(A-I s_{0}\right)^{k} B s^{k-1}\right]
$$

Alguns autores denominam a relação descrita na equação 76 como Aproximação de Padé.

Expandindo 76 em torno de $s_{0}=0$, encontra-se a função de transferência descrita como uma série de potências.

$$
G(s)=D-\lim _{k \rightarrow \infty}\left[C A^{-1} B-C A^{-2} B s-\cdots-C A^{-k-1} B s^{k}\right]=D+\sum_{k=0}^{\infty}-m_{k} s^{k}
$$

sendo que $m_{k}$ são os chamados momentos da função de transferência.

$$
m_{k}=C A^{-k-1} B \quad k=0,1,2, \ldots
$$

Na equação 77, o valor da resposta em estado estável é encontrado com apenas o primeiro momento da série e quanto mais termos são adicionados à série, maior será a ordem da função de aproximação. Como a aproximação é baseada em uma expansão de Taylor, é esperado que quanto maior o número de termos adicionados à série, melhor será a aproximação.

Substituindo $75 \mathrm{em} 74 \mathrm{em}$ torno de $s_{0} \rightarrow \infty$, encontra-se uma outra aproximação da função de transferência em série de potências.

$$
G(s)=D+\lim _{k \rightarrow \infty}\left[C B s^{-1}+C A B s^{-2}+\cdots+C A^{k-1} B s^{-k}\right]=D+\sum_{k=0}^{\infty} \bar{m}_{k} s^{-k}
$$

sendo que $\bar{m}_{k}$ são os chamados coeficientes de Markov do sistema.

$$
\bar{m}_{k}=C A^{k-1} B \quad k=1,2, \ldots
$$

Kailath mostra que o k-ésimo coeficiente de Markov é o valor da i-ésima derivada da resposta ao impulso do sistema em $t=0$ (KAILATH, 1980). 
Observa-se que o espaço formado pelas colunas de $M_{c}$, na equação 14, é igual ao espaço de Krylov na equação 73 truncado nos $n$ primeiros termos da série.

Salimbahrami e Lohmann (SALIMBAHRAMI; LOHMANN, 2002) provam que dado um sistema reduzido, truncado nos $m$ primeiros vetores da base de Krylov, sua função de transferência é igual à série da equação 77 truncada nos $m$ primeiros termos. Ou seja, a função de transferência do sistema reduzido de ordem $m$ possui os $m$ primeiros momentos iguais aos do sistema completo.

A medida que definimos um subespaço de Krylov de maior dimensão, os vetores calculados com a equação 77 são cada vez mais alinhados com o autovetor mais dominante da matriz $A$, este comportamento é mostrado de maneira esquemática na figura 5. Registros deste efeito numérico são encontrados em literatura (SCHILDERS; VORST; ROMMES, 2008) (LANCZOS, 1950) (FERRAZ, 2001).

A projeção para uma base de Krylov sem considerar a possível existência deste fenômeno pode levar à formação de uma base vetorial mal condicionada e o sistema projetado na nova base pode ter um comportamento numérico não desejado.

Figura 5 - Comportamento indesejado dos vetores que formam a base de Krylov.

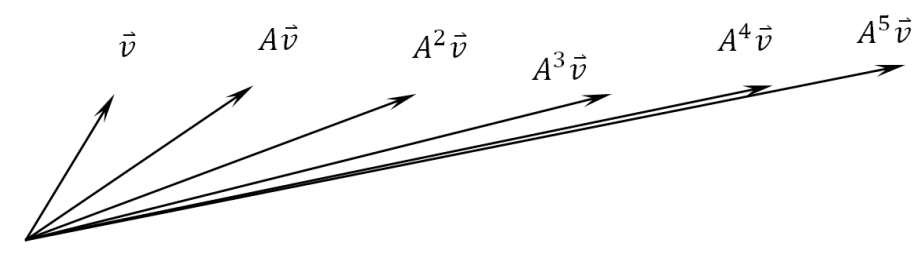

Para evitar o problema descrito acima, é conveniente escolher um espaço definido por vetores ortogonais que seja equivalente ao de Krylov. Uma das maneiras para se encontrar este conjunto de vetores é através da criação de uma base ortogonal de séries de potência, também conhecido como método de Arnoldi.

\subsubsection{Base ortonormal de séries de potência (Método de Arnoldi)}

Este método inicialmente proposto por Arnoldi (ARNOLDI, 1951) tem objetivo de encontrar uma base ortonormal à partir da base de Krylov.

Na Base de Krylov por método de Arnoldi (BKMA) são calculados um a um os vetores que irão compor a nova base através do algoritmo de Gram-Schmidt, ou seja, cada novo vetor é ortogonalizado em relação à todos os anteriores, que já compõem a base, e posteriormente normalizado. Este procedimento também é conhecido como Método de Arnoldi (ANTOULAS, 2005). 
O algoritmo proposto abaixo descreve o processo iterativo para encontrar uma base ortonormal de dimensão $m$. Saad (SAAD, 2003) mostra que a maior dimensão que a base calculada a partir do metodo de Arnoldi é igual à ordem da matriz $A$.

1. Eleger um vetor de início $w_{1}$

A escolha do vetor de início da base de Krylov tem grande influência no comportamento e representatividade do sistema reduzido em relação ao completo, portanto deve ser dedicada especial atenção à esta escolha (SALIMBAHRAMI; LOHMANN, 2002).

Deseja-se que o vetor $w_{1}$ de início da série esteja alinhado à uma direção que, quando multiplicado pela matriz $A$, tenha o maior ganho possível. De acordo com o método de DVS descrito no item 5.2, o primeiro vetor singular à esquerda de $A$ é o vetor que satisfaz este requisito. Havendo a decisão de não realizar o cálculo de um vetor singular, pode-se utilizar como alternativa para o método, o primeiro autovetor da matriz $A$.

2. Calcular o primeiro vetor da base normalizando $\omega_{1}$

$$
v_{1}=\frac{\omega_{1}}{\left\|\omega_{1}\right\|}
$$

3. Repetir os passos $a, b$ e $c$ para cada $\mathbf{j}=2,3, \ldots n$

a ) Ortogonalizar $A v_{j-1}$, que seria o próximo vetor da base, em relação todos os vetores anteriores que compõem a base (de acordo com a Figura 6):

$$
\omega_{j}=A v_{j-1}-\sum_{i=1}^{j-1}\left(\left(A v_{j-1}\right) \cdot v_{i}\right) v_{i}
$$

b ) Se o módulo de $\omega_{j}$ for nulo, parar o processo.

c) Normalizar $\omega_{j}$ para que tenha módulo unitário.

$$
v_{j}=\frac{\omega_{j}}{\left\|\omega_{j}\right\|}
$$

Após o procedimento acima, é montada a matriz $V_{k r}=\left[v_{1}, v_{2}, v_{3}, \ldots, v_{n}\right]$ cujas colunas são os vetores com compõem a base ortonormal de Krylov calculada pelo método de Arnoldi.

Pode-se observar que o espaço vetorial gerado pela base ortonormal $\left\{v_{1}, v_{2}, v_{3}, \ldots, v_{n}\right\}$ é equivalente ao espaço $K^{n}(A ; v)$ mostrado na equação 73 .

Aplicando uma mudança de base

$$
V_{k r} x_{k r}=x
$$


Figura 6 - Ortogonalização de um novo vetor da base de Krylov pelo método de Arnoldi.

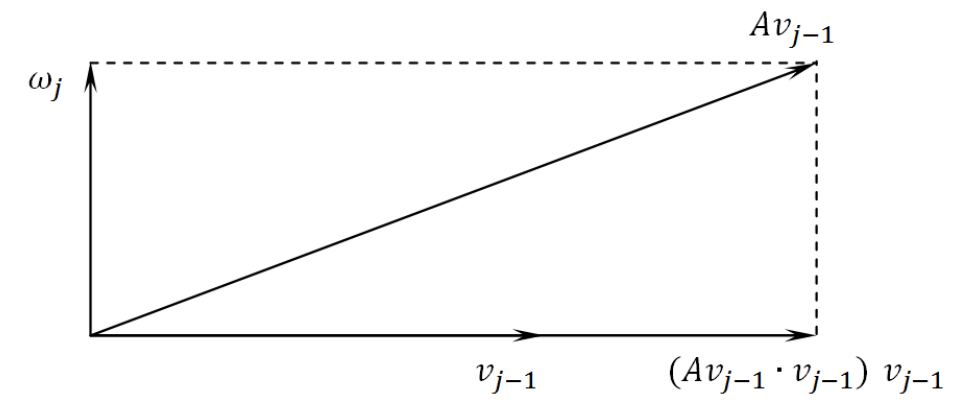

onde o índice $k r$ indica o vetor de estados na base ortonormal de Krylov calculada pelo método de Arnoldi. Desta maneira, pode-se escrever o sistema dinâmico na base de Krylov como:

$$
\left\{\begin{array}{c}
\dot{x}_{k r} \\
\hline y
\end{array}\right\}=\left[\begin{array}{c|c}
V_{k r}^{T} A V_{k r} & V_{k r}^{T} B \\
\hline C V_{k r} & D
\end{array}\right] \cdot\left\{\begin{array}{c}
x_{k r} \\
\hline u
\end{array}\right\}
$$

Uma característica dos métodos de projeção em bases de Krylov é a possibilidade de que o sistema reduzido seja instável mesmo que o sistema completo seja estável. Portanto a estabilidade do sistema reduzido deve ser verificada após o processo de redução.

Alguns métodos baseados na projeção em bases de Krylov foram desenvolvidos para que a estabilidade do sistema completo seja preservada no sistema reduzido (JAIMOUKHA; KASENALLY, 1997) e (ANTOULAS; SORENSEN; GUGERCIN, 2001).

Outras variações de métodos de projeção em bases de Krylov podem ser encontradas em (SALIMBAHRAMI; LOHMANN, 2002), (SALIMBAHRAMI; LOHMANN, 2006) e (BAI, 2002).

Um exemplo numérico da projeção de um sistema linear em uma base de Krylov obtida pelo Método de Arnoldi pode ser encontrada em anexo no item C.2.

\subsection{REALIZAÇÃO BALANCEADA}

Nesta base proposta por Moore (MOORE, 1981), diagonaliza-se os gramianos de controlabilidade e observabilidade do sistema. Para esta mudança de base, também conhecida como Realização Balanceada de Lyapunov (GUGERCIN; ANTOULAS, 2004), serão considerados sistemas dinâmicos LCl assintoticamente estáveis.

Para o caso de sistemas instáveis, a transformação para a base de realização balanceada deve ser realizada separadamente para as partes estáveis e instáveis do sistema (ANTOULAS, 2005) e (CHATURVEDI, 2011). Entretanto estes casos não serão abordados neste trabalho.

Dado um sistema estável representado como na equação 1 pode-se realizar uma transfor- 
mação de base do tipo

$$
w_{r b}=T_{r b} x
$$

onde o índice ${ }_{r b}$ indica o vetor numa base de realização balanceada e $T_{r b}$ é a matriz de transformação da base original para a base de realização balanceada.

Substituindo 86 em 1 . encontra-se:

$$
\left\{\begin{array}{c|c}
\dot{w}_{r b} \\
\hline y
\end{array}\right\}=\left[\begin{array}{c|c}
T_{r b} A T_{r b}^{-1} & T_{r b} B \\
\hline C T_{r b}^{-1} & D
\end{array}\right] \cdot\left\{\begin{array}{c}
w_{r b} \\
\hline u
\end{array}\right\}
$$

Como as entradas e saídas do sistema são invariantes na base que o mesmo é definido, as energias das funções de entrada e saída definidas respectivamente nas equações 16 e 17 também não variam quando há uma mudança de base. Desta maneira, substituindo 86 em 16. encontra-se:

$$
x^{*} L_{c}^{-1} x=w_{r b}^{*}\left(T_{r b} L_{c} T_{r b}^{*}\right)^{-1} w_{r b}
$$

Por comparação, conclui-se que existe uma relação entre os gramianos de controlabilidade na base original $L_{c}$ e na base balanceada $L_{c^{r b}}$, dada por:

$$
L_{c^{r b}}=T_{r b} L_{c} T_{r b}^{*}
$$

Analogamente, substituindo 86 em 17 , obtém-se:

$$
x^{*} L_{o} x=w_{r b}^{*}\left(\left(T_{r b}^{*}\right)^{-1} L_{o} T_{r b}^{-1}\right) w_{r b}
$$

e, também por comparação, conclui-se que existe uma relação entre o gramiano de observabilidade na base original $L_{o}$ e na base balanceada $L_{o^{r b}}$, dada por:

$$
L_{o^{r b}}=\left(T_{r b}^{*}\right)^{-1} L_{o} T_{r b}^{-1}
$$

O gramiano cruzado na base balanceada, definido pelo produto dos gramianos de controlabilidade e observabilidade, pode ser também escrito usando as relações 88 e 89 .

$$
L_{x^{r b}}^{2}=L_{c^{r b}} L_{o^{r b}}=T_{r b} L_{c} L_{o} T_{r b}^{-1}
$$

Se, de maneira arbitrária, os gramianos de controlabilidade e observabilidade na base balanceada forem considerados iguais e diagonais: $L_{c^{r b}}=L_{o^{r b}}=\Gamma_{r b}$, a partir da equação 90 pode-se diagonalizar o gramiano cruzado $L_{x^{r b}}$ na forma:

$$
L_{x}^{2}=L_{c} L_{o}=T_{r b}^{-1} \Gamma_{r b}^{2} T_{r b}
$$


Onde os valores da diagonal de $\Gamma_{r b}^{2}=\operatorname{diag}\left(\gamma_{1}^{2}, \ldots, \gamma_{n}^{2}\right)$ são os autovalores do quadrado do gramiano cruzado (como mostrado na equação 13) e $\Gamma_{r b}=\operatorname{diag}\left(\gamma_{1}, \ldots, \gamma_{n}\right)$ são chamados normas Hankel do sistema (ZHOU; DOYLE, 1998).

$$
L_{x^{r b}}^{2}=\Gamma_{r b}^{2}
$$

Os GDL do sistema são reorganizados de maneira que as normas Hankel do sistema na base balanceada estejam em ordem decrescente: $\gamma_{1} \geq \gamma_{2} \geq \cdots \geq \gamma_{n}$

Uma maneira de se calcular a matriz de transformação $T_{r b}$ é através do seguinte procedimento:

1. Realizar a decomposição de Cholesky (GOLUB; LOAN, 1996) para o gramiano de controlabilidade: $L_{c}=U_{r b} U_{r b}^{*}$

2. Realizar a decomposição da matriz $U_{r b}^{*} L_{o} U_{r b}$ em autovalores e autovetores: $U_{r b}^{*} L_{o} U_{r b}=$ $K_{r b} \Gamma_{r b} K_{r b}^{*}$

3. A matriz $\Gamma_{r b}$ pode ser calculada a partir das relações

$$
\begin{gathered}
\Gamma_{r b}^{\frac{1}{2}} K_{r b}^{*} U_{r b}^{-1} L_{c} U_{r b}^{-*} K_{r b} \Gamma_{r b}^{\frac{1}{2}}=\Gamma_{r b}^{\frac{1}{2}} K_{r b}^{*} K_{r b} \Gamma_{r b}^{\frac{1}{2}}=\Gamma_{r b} \\
\Gamma_{r b}^{-\frac{1}{2}} K_{r b}^{*} U_{r b}^{*} L_{o} U_{r b} K_{r b} \Gamma_{r b}^{-\frac{1}{2}}=\Gamma_{r b}^{-\frac{1}{2}} K_{r b}^{*} K_{r b} \Gamma_{r b} K_{r b}^{*} K_{r b} \Gamma_{r b}^{-\frac{1}{2}}=\Gamma_{r b}
\end{gathered}
$$

Observando as equações 89 , 90 e 93 a matriz de transformação de base $T_{r b}$ é, por comparação:

$$
\begin{aligned}
& T_{r b}=\Gamma_{r b}^{\frac{1}{2}} K_{r b}^{*} U_{r b}^{-1} \\
& T_{r b}^{-1}=U_{r b} K_{r b} \Gamma_{r b}^{-\frac{1}{2}}
\end{aligned}
$$

Cabe lembrar que a base de realização balanceada sempre existe para sistemas em realização mínima e estáveis segundo o critério de estabilidade de Lyapunov, ou seja, com autovalores com parte real não positiva. 


\section{CRITÉRIOS DE SELEÇÃO}

A seleção das variáveis a serem eliminadas é um fator diretamente relacionado com a capacidade do sistema reduzido em reproduzir características do sistema completo. Esta seleção pode ser realizada através do conhecimento prévio do analista sobre o comportamento do sistema, entretanto esta prática pode se tornar inviável para sistemas de alta ordem.

Neste capítulo, serão apresentados e discutidos critérios para a seleção das variáveis que são boas candidatas à eliminação. Para cada critério, é estabelecido um parâmetro de seleção que atribui um valor escalar a cada GDL, que quantifica a importância de se preservar este GDL no modelo reduzido.

Desta maneira, o vetor de estados do sistema completo deve ser rearranjado de maneira que os valores do parâmetro de seleção sejam decrescentes. Portanto os primeiros GDL no vetor de estados serão correspondentes a maiores valores do parâmetro de seleção e os últimos correspondem a menores valores do mesmo parâmetro.

Posteriormente, no capítulo 7] serão apresentadas metodologias para a eliminação de GDL do sistema completo.

\subsection{CRITÉRIOS DE SELEÇÃO EXCLUSIVOS PARA BASE MODAL}

Neste trabalho, os critérios exclusivos para a base modal atribuem um valor de parâmetro de seleção para cada modo de vibrar, o que corresponde a um ou dois GDL do sistema de primeira ordem (respectivamente para autovalores reais ou complexos conjugados).

A partir da equação 43 pode-se descrever isoladamente a dinâmica de um modo de vibrar, ou seja, a projeção da resposta do sistema no subespaço definido por suas formas modais:

$$
\ddot{z}_{i}+2 \omega_{n i} \zeta_{i} \dot{z}_{i}+\omega_{n i}^{2} z_{i}=0
$$

Onde $z_{i}, \omega_{n i}$ e $\zeta_{i}$ são chamados respectivamente de resposta modal, frequência natural não amortecida e fator de amortecimento do i-ésimo modo de vibrar do sistema.

$\mathrm{O}$ autovalor correspondente ao i-ésimo autovetor pode ser escrito em função de $\zeta_{i}$ e $\omega_{n i}$ e representado graficamente como na figura 7

$$
\lambda_{i}=-\omega_{n i} \zeta_{i} \pm i \omega_{n i} \sqrt{1-\zeta_{i}^{2}}
$$

O autor expõe a seguir 4 critérios de seleção que podem ser utilizados na base modal

- Critério de seleção baseado na frequência natural (CSF) 
Figura 7 - Autovalor de um sistema dinâmico no plano complexo.

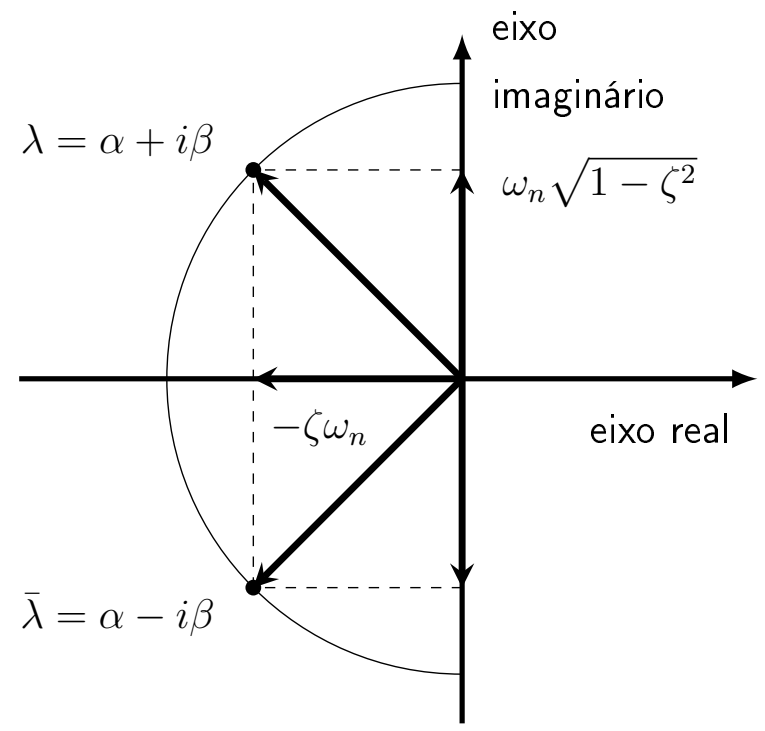

- Critério de seleção baseado na frequência e fator de amortecimento (CSFA)

- Critério de seleção baseado na observabilidade e controlabilidade (CSCO)

- Critério de seleção baseado na função de transferêcia (CSFT)

\subsubsection{Critério de seleção baseado na frequência natural (CSF)}

Um critério de seleção bastante usado para a base modal é descrito em (GAWRONSKI, 1998), onde o parâmetro $P_{\omega_{n}}$ é o inverso da frequência natural $\omega_{n k}$ do k-ésimo modo de vibrar do sistema.

$$
P_{\omega_{n}}^{(k)}=\frac{1}{\omega_{n k}}=\frac{1}{\sqrt{\alpha_{k}^{2}+\beta_{k}^{2}}}
$$

Uma interpretação geométrica para o parâmetro de seleção $P_{\omega_{n}}$ é que os polos candidatos à eliminação estão fora de circunferência de raio $\omega_{n}$ e com centro em $(0,0)$, conforme indicado pela figura 8 .

Uma desvantagem deste método é que modos com baixo fator de amortecimento e maiores frequências naturais (que seriam bons candidatos a serem eliminados segundo este critério) podem ter contribuição importante na resposta transiente do sistema. Portanto com a utilização deste critério, a similaridade da resposta transitória do SR em relação ao SC pode ser comprometida. 
Figura 8 - Critério de seleção baseado na frequência natural dos autovalores

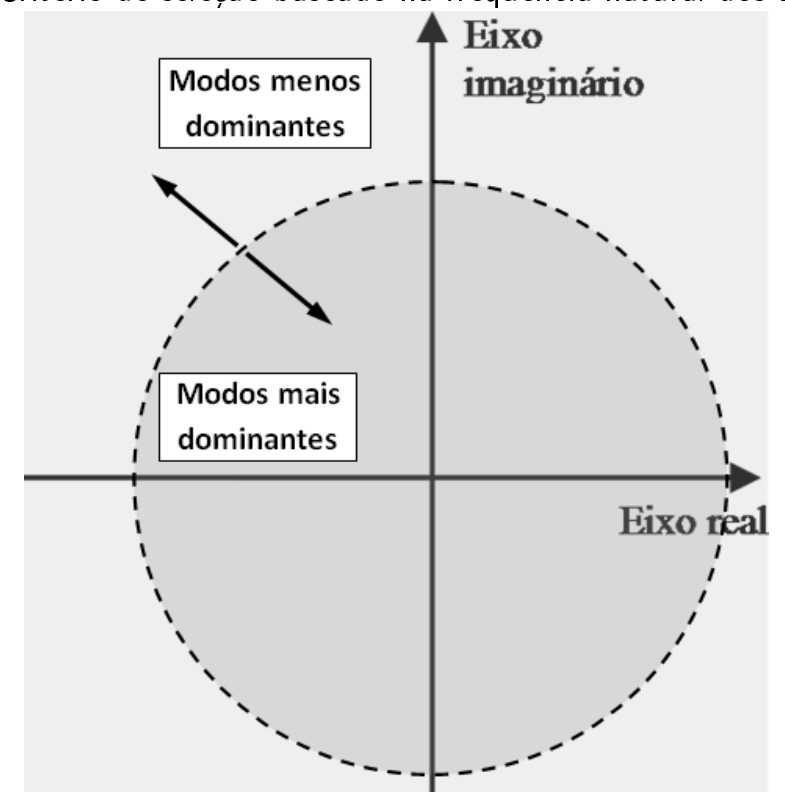

\subsubsection{Critério de seleção baseado na frequência natural e fator de amortecimento (CSFA)}

Este critério, descrito por (OBINATA; ANDERSON, 2001), para seleção dos modos candidatos à eliminação, é baseado no fator de amortecimento $\zeta$ e frequência não amortecida $\omega_{n}$ de cada modo de vibrar.

Sabendo que o produto $\omega_{n} \zeta$ está diretamente relacionado com a potência dissipada do modo, este critério penaliza modos de altos fatores de amortecimento e altas frequências naturais. $O$ parâmetro de seleção $P_{a f}$ para um modo é o inverso do produto do fator de amortecimento do modo com sua frequência natural correspondente.

$$
P_{a f}^{(k)}=\frac{1}{-\operatorname{Re}\left(\lambda_{k}\right)}=\frac{1}{-\alpha_{k}}=\frac{1}{\omega_{n k} \zeta_{k}}
$$

Também é sabido que o produto do amortecimento de um modo com sua frequência natural é igual à parte real do autovalor correspondente (OGATA, 2001). Portanto o parâmetro de seleção $P_{a f}$ também pode ser calculado através do inverso da parte real do autovalor correspondente ao modo de vibrar.

A figura 9 mostra uma representação gráfica dos modos mais dominantes e menos dominantes do sistema (estes últimos candidados à eliminação).

Outro ponto importante para salinetar é que, para uma entrada degrau, o tempo de acomodação $t_{s k}$ do k-ésimo modo de vibrar é: (OGATA, 2001)

$$
t_{s k}=\frac{4}{\omega_{n k} \zeta_{k}}
$$


Figura 9 - Critério de seleção baseado na parte real dos autovalores

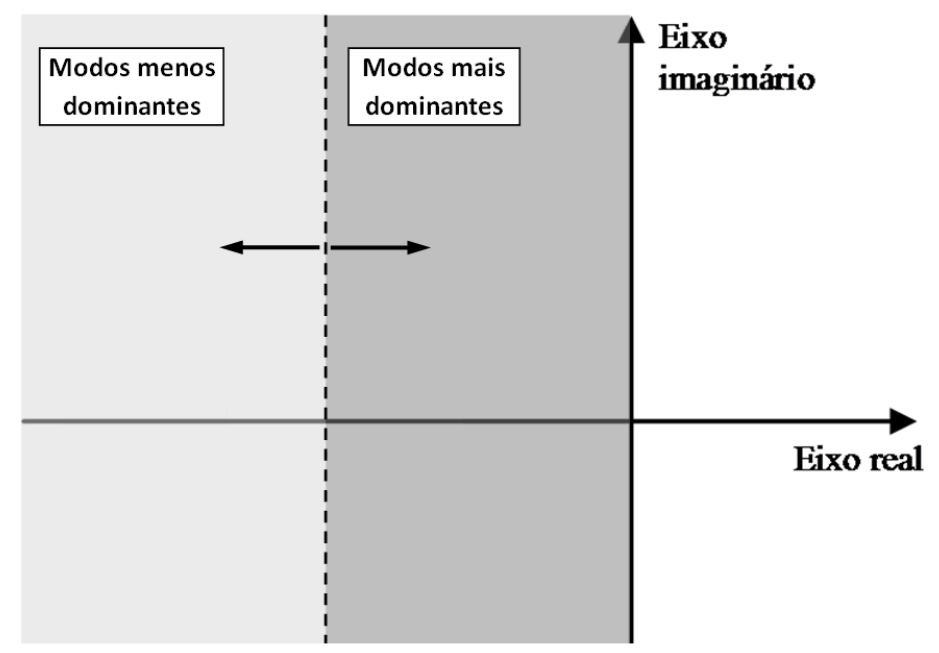

Portanto segundo este critério, os modos que são bons candidatos a serem eliminados tem tempo de acomodação pequeno, ou seja, estabilizam mais rapidamente na condição de estado estável que outros modos. Isso reforça o conceito de que este critério preserva modos com maior contribuição para a resposta transiente do sistema.

Conclui-se que este critério é aconselhado para situações onde deseja-se priorizar a preservação do comportamento transiente do sistema, como por exemplo quando submetido à excitações não periódicas.

\subsubsection{Critério de seleção baseado na controlabilidade e observabilidade (CSCO)}

Outro critério de seleção parte do pressuposto que os valores das normas Hankel dos modos de vibrar são boas aproximações para as normas Hankel do sistema completo, em especial para sistemas fracamente amortecidos (GREGORY, 1984) e (PERNEBO; SILVERMAN, 1982). Como as normas Hankel de um sistema dinâmico indicam o quanto este sistema é simultaneamente controlável e observável, pode-se estabelecer um critério que selecione os modos de vibrar menos controláveis e observáveis para serem eliminados.

O autor se utilizará de um abuso de linguagem e neste trabalho as normas Hankel calculadas a partir dos sistemas desacoplados que descrevem a dinâmica dos modos de vibrar serão chamadas de normas Hankel dos modos de vibrar.

Neste critério, descrito por Varga em (VARGA, 1993), o sistema completo projetado na base modal é separado em subsistemas desacoplados que descrevem a dinâmica dos modos de vibrar, como na equação 45. As normas Hankel destes subsistemas são calculadas e a maior 
norma de cada modo é usada como critério de seleção.

$$
P_{\gamma m}^{(k)}=\max _{i} \gamma_{i}^{(k)} \quad k=1,2, \ldots, n
$$

Onde $\gamma_{i}^{(k)}$ são as normas Hankel do k-ésimo subsistema e $P_{\gamma m}^{(k)}$ é o parâmetro de seleção baseado na controlabilidade e observabilidade dos modos de vibrar.

A figura 10 mostra uma comparação entre as normas Hankel de um sistema completo e dos modos de vibrar.

Figura 10 - Exemplo de normas Hankel aproximadas pelos blocos da base modal.

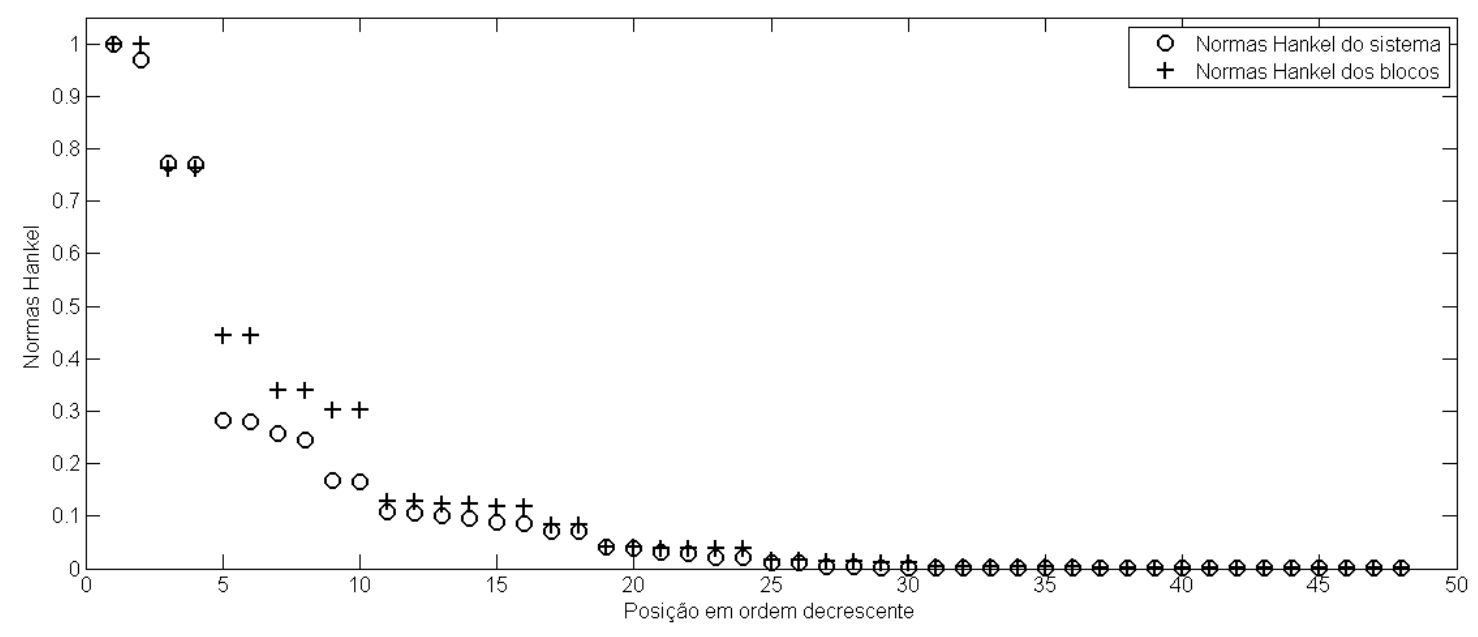

É importante lembrar que apesar de serem atribuídas normas Hankel aos blocos do sistema da base modal, os modos de vibrar não são equivalentes às direções de projeção da base de realização balanceada. Apesar disso, diversas fontes na literatura mostram que o parâmetro $P_{\gamma m}^{(k)}$ é uma boa alternativa para avaliar se um modo de vibrar é simultaneamente controlável e observável (GAWRONSKI, 1998).

\subsubsection{Critério de seleção baseado na função de transferêcia (CSFT)}

A partir da função de transferência do sistema completo projetado na base modal, descrita pelas equações 60 e 63 pode-se perceber que alguns termos da função de transferência têm maior participação que outros na resposta do sistema completo. Se a participação de um modo na função de transferência for pequena, este modo é pouco observável ou pouco controlável.

Desta maneira, pode-se selecionar os termos com menor contribuição como candidatos a serem eliminados.

A partir da equação 63, pode-se escrever, para sistemas não amortecidos (ou com amorte- 
cimento proporcional), a função de transferência modal $G_{k}(s)$ é

$$
G_{k}(s)=\left[\begin{array}{ccc}
\frac{\tilde{c}_{1, k} \tilde{b}_{k, 1}}{s-\lambda_{k}} & \cdots & \frac{\tilde{c}_{1, k} \tilde{b}_{k, n_{u}}}{s-\lambda_{k}} \\
\vdots & \ddots & \\
\frac{\tilde{c}_{n_{y}, k} \tilde{b}_{k, 1}}{s-\lambda_{k}} & & \frac{\tilde{c}_{n_{y}, k} \tilde{b}_{k, n_{u}}}{s-\lambda_{k}}
\end{array}\right]
$$

Quando o valor de $\tilde{c}_{i, k}$ é muito pequeno, pode-se dizer que o respectivo modo do sistema é pouco observável na saída $i$. O mesmo ocorre quando $\tilde{b}_{k, j}$ é pequeno e o modo é pouco controlável pela entrada $j$. Desta maneira, são considerados pouco controláveis ou pouco observáveis (para a saída $i$ e entrada $j$ ) os modo cujos produto $\tilde{c}_{i, k} \tilde{b}_{k, j}$ (também chamado de resíduo da função de transferência) é significativamente pequeno.

Baseando-se na discussão acima, conclui-se que a função de transferência modal $G_{k}(s)$ pode ser usada para determinar a influência de um modo na resposta do sistema completo.

Partindo para o caso mais genérico de sistemas com amortecimento não proporcional, a função de transferência modal $G_{k}(s)$ é

$$
G_{k}(s)=\left[\begin{array}{cc}
\tilde{c}_{1,2 k-1} & \tilde{c}_{1,2 k} \\
\vdots & \\
\tilde{c}_{n_{y}, 2 k-1} & \tilde{c}_{n_{y}, 2 k}
\end{array}\right] \cdot\left[\Lambda_{k}^{-1}(s)\right] \cdot\left[\begin{array}{ccc}
\tilde{b}_{2 k-1,1} & \ldots & \tilde{b}_{2 k-1, n_{u}} \\
\tilde{b}_{2 k, 1} & \ldots & \tilde{b}_{2 k, n_{u}}
\end{array}\right]
$$

Uma maneira de estimar o quão pequenos são os valores das componentes de $G_{k}(s)$ é calcular os valores singulares desta matriz em uma frequência de interesse $s_{0}$. A função de transferência modal $G_{k}\left(s_{0}\right)$ com pequenos ganhos terá menor participação na resposta da função de transferência $G\left(s_{0}\right)$ do sistema completo em comparação à outros modos.

Define-se portanto o parâmetro de seleção $P_{s}$ baseado na controlabilidade e observabilidade, para seleção de modos de vibrar candidatos à eliminação. O valor de $P_{s}^{(k)}$ é gual ao maior ganho (ou maior valor singular) da função de transferência de um modo avaliado em uma frequência de interesse $s_{0}$ :

$$
P_{s}^{(k)}=\sigma_{\max }\left(G_{k}\left(s_{0}\right)\right)
$$

Onde $P_{s}^{(k)}$ é o parâmetro de seleção referente ao k-ésimo modo de vibrar.

Como exemplo, observando as funções de transferência modais $G_{k}(s)$ na figura 3 e assumindo que o sistema em questão seja SISO, é possivel ver que segundo o critério acima, o modo que mais contribui para a resposta do sistema completo entre 1 e $7 \mathrm{~Hz}$ é o modo 1 . Já o modo 3 é o que mais contribui para a resposta do sistema completo entre 11 e $21 \mathrm{~Hz}$ segundo o mesmo critério. 
Este critério prioriza manter os modos de vibração que tem maior participação na resposta do sistema completo em torno de uma frequência $s_{0}$. Portanto ele é recomendado quando o sistema reduzido deve reproduzir a resposta do sistema completo quando submetido à excitações periódicas em um intervalo de frequência conhecido.

\subsection{CRITÉRIO DE SELEÇÃO BASEADO EM VALORES SINGULARES (CSVS)}

Baseado na base de vetores singulares descrita no item 5.2. o critério de seleção baseado em valores singulares (CSVS) estabelece que o sistema reduzido deve preservar os maiores ganhos da matriz $A$ do sistema completo, desprezando as direções onde os ganhos sejam menores. Como valores singulares nulos correspondem à vetores singulares que compoem um subespaço nulo, este critério assume que ganhos menores que um valor arbitrário são correspondentes a um subespaço que é considerado aproximadamente nulo para fins da redução da ordem do modelo.

Assumindo que a somatória descrita na equação 69 está ordenada de forma decrescente de valores singulares, isso indica que os maiores ganhos da transformação linear $A$ estão nos primeiros termos da somatória. Desta maneira, deseja-se eliminar os $r$ últimos termos desta somatória, onde $n_{r}=n-r$. Em outras palavras, os termos com menores ganhos serão desprezados e considerados como ganho nulo.

$$
A=U\left[\begin{array}{cccccc}
\sigma_{1} & & & & & \\
& \ddots & & & & \\
& & \sigma_{n_{r}} & & & \\
& & & \sigma_{\left(n_{r}+1\right)} & & \\
& & & \ddots & \\
& & & & \sigma_{n}
\end{array}\right] \bar{V}^{T}
$$

Portanto o critério de seleção $P_{\sigma}^{(k)}$ dos GDL que são bons candidatos a eliminação, baseado nos valores singulares da matriz $A$ do sistema é:

$$
P_{\sigma}^{(k)}=\sigma_{k}
$$

\subsection{CRITÉRIO DE SELEÇÃO POR VETORES NA BASE DE KRYLOV (CSVK)}

Assumindo que o sistema completo seja projetado em uma base de Krylov, descrita no item 5.3. pode-se estabelecer um critério de seleção baseado nos vetores que compõem o espaço definido na equação 73 . 


$$
K^{i}(A ; v)=\operatorname{span}\left\{v, A v, A^{2} v, \ldots, A^{i-1} v\right\}
$$

No CSVK, os graus de liberdade associados aos primeiros vetores da base são bons candidatos a serem preservados no sistema reduzido.

A motivação deste critério é o fato de que para o caso específico do espaço de Krylov ser calculado através do método de Arnoldi, os $n_{r}$ primeiros coeficientes de Markov $\bar{m}_{k}$ dos sistemas completo e reduzido por truncamento direto são iguais (ANTOULAS; SORENSEN; GUGERCIN, 2001). Onde $n_{r}$ é a ordem do sistema reduzido.

O método de truncamento direto citado no parágrafo anterior será descrito mais adiante no item 7.2 .

\subsection{CRITÉRIO DE SELEÇÃO BASEADO EM NORMAS HANKEL (CSNH)}

O critério de seleção baseado em normas Hankel (CSNH) é baseado no trabalho realizado por Moore (MOORE, 1981), onde os GDL que são bons candidatos a eliminação são selecionados de acordo com as normas Hankel do sistema projetado na base de realização balanceada.

Um sistema dinâmico em sua realização não mínima possui componentes não controláveis ou não observáveis, que por sua vez podem ser eliminadas do modelo sem que exista perda de quaisquer informações sobre seu comportamento. Sendo assim, componentes fracamente controláveis ou observáveis de um sistema em sua realização mínima são bons candidatos à eliminação durante o processo de redução do mesmo pois têm pouca contribuição na resposta do sistema (ANTOULAS, 2005).

Ao se diagonalizar os gramianos de controlabilidade e observabilidade, valores muito pequenos na diagonal principal (em relação aos maiores valores da mesma) correspondem à componentes pouco controláveis ou pouco observáveis respectivamente. Uma maneira de buscar componentes simultaneamente pouco controláveis e pouco observáveis é diagonalizar o produto entre os dois gramianos citados, que é igual ao quadrado do gramiano cruzado, descrito na equação 11 .

Neste caso, a diferença entre os maiores e menores valores da diagonal principal do gramiano cruzado diagonalizado (e a taxa que estes valores diminuem quando colocados em ordem decrescente) tem grande importância na representatividade do modelo reduzido. Quanto maior a taxa de decaimento das normas Hankel, maior será a contribuição dos GDL associados a altos valores de normas Hankel na resposta do sistema (e consequentemente menor será a contribuição das componentes associadas a pequenos valores de normas Hankel na resposta do sistema). 
Baseado na mudança para uma base balanceada, descrita no item 5.4, sabe-se que o gramiano cruzado já se encontra diagonalizado, conforme equação 92 Portanto os valores da diagonal principal do gramiano cruzado na base balanceada, também conhecidas como normas Hankel, são indicadores que quantificam o quanto um DGL é simultaneamente controlável e observável.

Esta configuração favorece a seleção de componentes do sistema que sejam fracamente controláveis e observáveis simultaneamente, pois correspondem à pequenos valores da diagonal principal do gramiano cruzado. Sendo assim, os GDL na base de realização balanceada estão organizadas por ordem das mais controláveis e observáveis para as menos controláveis e observáveis.

Portanto no CSNH, define-se o parâmetro de seleção $P_{\gamma}$, específico para sistemas projetados na base de realização balanceada, que é baseado nos valores das normas Hankel do mesmo, calculados segundo a equação 13 .

$$
P_{\gamma}^{(k)}=\gamma_{k} \quad k=1,2, \ldots, n
$$

Como pode-se esperar, quanto maior a taxa de decaimento dos valores das normas Hankel, maior será diferença entre as normas Hankel das componentes mantidas e as eliminadas. Consequentemente, as variáveis eliminadas serão fracamente controláveis e observáveis em relação às mantidas no modelo reduzido. Esforços foram feitos para estimar a taxa de decaimento das normas Hankel sem a necessidade de calculá-las, pois isso demanda alto custo computacional $\left(\mathcal{O}\left(n^{3}\right)\right.$ ). Antoulas (ANTOULAS; SORENSEN; ZHOU, 2002) discute os métodos disponíveis para estimar esta taxa de decaimento, assim como vantagens e desvantagens de cada um. 


\section{MÉTODOS DE ELIMINAÇÃO}

Neste capítulo, serão apresentados alguns métodos para realizar a eliminação de GDL do sistema dinâmico completo, bem como suas particularidades, vantagens de desvantagens. A escolha dos GDL que serão eliminadas é feita segundo critérios de seleção, detalhados no capítulo 6

É assumido que a redução de ordem do sistema completo é não trivial, ou seja, o sistema completo está em sua realização mínima. Para um sistema dinâmico linear, dada uma realização não mínima de ordem $\bar{n}$, existe uma realização de ordem $n$ dita mínima, tal que $n<\bar{n}$, que descreve o sistema com o mínimo número de estados.

\subsection{ELIMINAÇÃO POR CONDENSAÇÃO ESTÁTICA}

Existem aplicações onde deseja-se calcular o modelo reduzido de um sistema para encontrar sua solução estática. As técnicas de eliminação por condensação estática se destacam em situações onde há a necessidade de calcular uma grande quantidade de soluções estáticas, onde apenas uma parcela do sistema tem seus valores modificados e outra se mantém constante. Esta situação ocorre por exemplo em alguns tipos de otimizações.

As equações 107 a 112 foram apresentadas segundo Cook (COOK, 1994).

Sistemas estáticos como da equação 23 podem ser escritos na seguinte forma:

$$
\left[\begin{array}{ll}
A_{11} & A_{12} \\
A_{21} & A_{22}
\end{array}\right] \cdot\left\{\begin{array}{l}
x_{1} \\
x_{2}
\end{array}\right\}=\left\{\begin{array}{l}
F_{1} \\
F_{2}
\end{array}\right\}
$$

Onde o vetor $x=\left(x_{1}, x_{2}\right)^{T}$ está separado em duas parcelas. A primeira com os GDL que devem ser preservadas no sistema reduzido. Já a segunda parte, contempla os GDL que têm importância secundária para os objetivos determinados para a análise do sistema.

Pode-se isolar o vetor $x_{2}$ da segunda linha da equação 107

$$
x_{2}=A_{22}^{-1} F_{2}-A_{22}^{-1} A_{21} x_{1}
$$

Observa-se na equação 108 que os valores do vetor $x_{2}$ são compostos pela soma de dois termos. O primeiro termo é oriundo das forças externas ao sistema, que atuam diretamente nas coordenadas $x_{2}$. Já o segundo termo é oriundo das forças internas do sistema, causadas pelos deslocamentos de $x_{1}$, que podem afetar o equilíbrio do sistema e consequentemente os valores de $x_{2}$. 
Substituindo a equação 108 na primeira linha da equação 107 , obtém-se:

$$
\left(A_{11}-A_{12} A_{22}^{-1} A_{21}\right) x_{1}=F_{1}-A_{12} A_{22}^{-1} F_{2}
$$

A partir da equação 109, pode-se relacionar o vetor de GDL do sistema completo com o do sistema reduzido. Desta forma, quando calculada a solução do sistema reduzido, pode-se recuperar a solução exata do sistema completo através da seguinte equação:

$$
\left\{\begin{array}{l}
x_{1} \\
x_{2}
\end{array}\right\}=\left[\begin{array}{c}
I \\
-A_{22}^{-1} A_{21}
\end{array}\right] x_{1}+\left[\begin{array}{c}
0 \\
A_{22}^{-1} F_{2}
\end{array}\right]
$$

Portanto pode-se definir a matriz reduzida $A_{r}$ e vetor reduzido de forças generalizadas $F_{r}$ para o Método de Guyan como

$$
\begin{aligned}
& A_{r}=A_{11}-A_{12} A_{22}^{-1} A_{21} \\
& F_{r}=F_{1}-A_{12} A_{22}^{-1} F_{2}
\end{aligned}
$$

com

$$
A_{r} x_{r e q}=F_{r}
$$

\subsubsection{Eliminação por método de Guyan}

Neste método proposto por Guyan (GUYAN, 1965), considera-se que as forças externas ao sistema atuam diretamente apenas nos GDL mantidos no sistema reduzido, ou seja, que correspondem ao vetor $x_{1}$ na equação 107 .

Considerando que o vetor $F_{2}$ é nulo na equação 107 pode-se escrever a equação 110 na forma

$$
x=T_{g} x_{1}
$$

onde

$$
T_{g}=\left[\begin{array}{c}
I \\
-A_{22}^{-1} A_{21}
\end{array}\right]
$$

A energia potencial no sistema definido pela equação 107 pode ser escrita na forma (MElROVITCH, 1980):

$$
E_{p}=\frac{1}{2} x^{T} A x
$$


Substituindo a equação 113 em 114 obtém-se a energia potencial do sistema completo apenas em função dos GDL do sistema reduzido:

$$
E_{p}=\frac{1}{2} x_{1}^{T} T_{g}^{T} A T_{g} x_{1}
$$

Expandindo os termos da equação 115 percebe-se que

$$
T_{g}^{T} A T_{g}=A_{r}=A_{11}-A_{12} A_{22}^{-1} A_{21}
$$

Portanto a energia potencial do sistema completo permanece igual após a redução (assumindo a hipótese de $\left.F_{2}=0\right)$.

$$
E_{p}=\frac{1}{2} x^{T} A x=\frac{1}{2} x_{r}^{T} A_{r} x_{r}
$$

Conclui-se que a solução da equação 109 é igual à parcela $x_{1}$ da solução da equação 107. Portanto o método descrito acima resulta em um sistema estático reduzido com solução exatamente igual à do completo. Após a solução do sistema reduzido, pode-se recuperar o valor das coordenadas $x_{2}$ desconsideradas durante o processo de redução através da equação 108.

Em aplicações de sistemas dinâmicos descritos como na equação 26, a energia cinética total no sistema é

$$
E_{c}=\frac{1}{2} \dot{x}^{T} M \dot{x}
$$

Para aplicar a mesma transformação de variáveis realizada anteriormente, substitui-se 113 em 118 Desta maneira, encontra-se uma aproximação da energia cinética que é análoga à equação 115 (GUYAN, 1965).

$$
E_{c r}=\frac{1}{2} \dot{x}_{r e q}^{T} T_{g}^{T} M T_{g} \dot{x}_{r e q}
$$

Desta maneira, a matriz de massa, separada por quatro submatrizes

$$
M=\left[\begin{array}{ll}
M_{11} & M_{12} \\
M_{21} & M_{22}
\end{array}\right]
$$

pode ser reduzida na forma

$$
\begin{aligned}
& M_{r}=T_{g}^{T} M T_{g} \\
& M_{r}=M_{11}-M_{21} A_{22}^{-1} A_{21}-\left(A_{22}^{-1} A_{21}\right)^{T}\left(M_{21}-M_{22} A_{22}^{-1} A_{21}\right)
\end{aligned}
$$

Ao contrário da redução para problems estáticos, onde a solução é exata, a utilização do método de Guyan para sistemas dinâmicos apresenta uma solução aproximada, porém 
não exata, do sistema completo. Isso pode ser percebido a partir da equação 120 onde há parcelas oriundas da rigidez do sistema que são usadas para compor o modelamento da inércia do sistema reduzido. Isto é consequência da necessidade de se manter a consistência na transformação de coordenadas realizada na equação 113 .

Variações do método originalmente proposto por Guyan podem ser encontradas em (PAZ, 1983) e (PAZ, 1989).

\subsubsection{Eliminação por método de Guyan Modificado}

No método de Guyan modificado, utiliza-se uma outra abordagem para a consendação estática, escrevendo a equação 107 na forma

$$
\left[\begin{array}{ll}
\hat{A}_{1} & \hat{A}_{2}
\end{array}\right]\left\{\begin{array}{l}
x_{1} \\
x_{2}
\end{array}\right\}=\hat{F}
$$

onde

$$
\begin{aligned}
& \hat{A}_{1}=\left(A_{11}^{T}, A_{21}^{T}\right)^{T} \\
& \hat{A}_{2}=\left(A_{12}^{T}, A_{22}^{T}\right)^{T} \\
& \hat{F}=\left(F_{1}^{T}, F_{2}^{T}\right)^{T}
\end{aligned}
$$

Consequentemente, a parcela $x_{2}$ é igual a

$$
x_{2}=\left(\hat{A}_{2}^{T} \hat{A}_{2}\right)^{-1}\left(\hat{A}_{2}^{T} \hat{F}-\hat{A}_{2}^{T} \hat{A}_{1} x_{1}\right)
$$

Substituindo 123 em 121, encontra-se uma outra maneira de calcular um sistema reduzido, diferente da equação 111 .

$$
\left[A_{11}-A_{12}\left(\hat{A}_{2}^{T} \hat{A}_{2}\right)^{-1} \hat{A}_{2}^{T} \hat{A}_{1}\right] x_{1}=F_{1}-A_{12}\left(\hat{A}_{2}^{T} \hat{A}_{2}\right)^{-1} \hat{A}_{2}^{T} \hat{F}
$$

Com

$$
\hat{A}_{2}^{T} \hat{A}_{2}=A_{12}^{T} A_{12}+A_{22}^{T} A_{22}
$$

Portanto pode-se definir a matriz reduzida $A_{r}$ e vetor reduzido de forças generalizadas $F_{r}$ para o Método de Guyan Modificado como

$$
\begin{aligned}
& A_{r}=\left[A_{11}-A_{12}\left(A_{12}^{T} A_{12}+A_{22}^{T} A_{22}\right)^{-1}\left(A_{12}^{T} A_{11}+A_{22}^{T} A_{21}\right)\right] \\
& F r=F_{1}-A_{12}\left(A_{12}^{T} A_{12}+A_{22}^{T} A_{22}\right)^{-1}\left(A_{12}^{T} F_{1}+A_{22}^{T} F_{2}\right)
\end{aligned}
$$


onde

$$
A_{r} x_{r e q}=F_{r}
$$

A partir da solução do sistema reduzido, pode-se encontrar a resposta do sistema completo a partir da seguinte relação:

$$
\begin{aligned}
& \left\{\begin{array}{l}
x_{1} \\
x_{2}
\end{array}\right\}=\left[\begin{array}{c}
I \\
-\left(A_{12}^{T} A_{12}+A_{22}^{T} A_{22}\right)^{-1}\left(A_{12}^{T} A_{11}+A_{22}^{T} A_{21}\right)
\end{array}\right] x_{r e q} \\
& +\left[\begin{array}{c}
0 \\
\left(A_{12}^{T} A_{12}+A_{22}^{T} A_{22}\right)^{-1}\left(A_{12}^{T} F_{1}+A_{22}^{T} F_{2}\right)
\end{array}\right]
\end{aligned}
$$

Observando as equações 111 e 126 observa-se que um dos critérios de escolha entre o Método de Guyan e Método de Guyan Modificado é a viabilidade de se inverter a matriz $A_{22}$. Caso $A_{22}$ não seja inversível, o Método de Guyan Modificado pode ser utilizado se $\left(A_{12}^{T} A_{12}+A_{22}^{T} A_{22}\right)$ for inversível (por ser uma soma de matrizes positivas semi-definidas, o resultado também é uma matriz positiva semi-definida e com maior chance de ser inversível).

Uma desvantagem do Método de Guyan Modificado é que a energia potencial do sistema completo $E_{p}$ não é sempre igual à do modelo reduzido.

Exemplos numéricos de eliminação de GDL pelos métodos de Guyan e Guyan modificado podem ser encontrados em anexo no iten C.3.

\subsection{ELIMINAÇÃO POR TRUNCAMENTO DIRETO (ETD)}

O método de eliminação por truncamento direto (ETD) consiste em desconsiderar as variáveis de estado menos representativas do sistema, que pode ser escrito na forma

$$
\left[\begin{array}{cc|c}
A_{11} & A_{12} & B_{1} \\
A_{21} & A_{22} & B_{2} \\
\hline C_{1} & C_{2} & D
\end{array}\right] \cdot\left\{\begin{array}{c}
x_{1} \\
x_{2} \\
\hline u
\end{array}\right\}=\left\{\begin{array}{c}
\dot{x}_{1} \\
\dot{x}_{2} \\
\hline y
\end{array}\right\}
$$

No método ETD, as variáveis menos representativas do sistema podem ser consideradas nulas $x_{2}=0$, fazendo com que:

$$
\left\{\begin{array}{l}
A_{r}=A_{11} \\
B_{r}=B_{1} \\
C_{r}=C_{1} \\
D_{r}=D
\end{array}\right.
$$


O sistema reduzido é escrito como:

$$
\left[\begin{array}{c|c}
A_{11} & B_{1} \\
\hline C_{1} & D
\end{array}\right] \cdot\left\{\begin{array}{c}
x_{r} \\
\hline u
\end{array}\right\}=\left\{\begin{array}{c}
\dot{x}_{r} \\
\hline y_{r}
\end{array}\right\}
$$

Portanto a função de transferência do sistema reduzido passa a ser

$$
G_{r}(s)=C_{1}\left(s I-A_{11}\right)^{-1} B_{1}+D
$$

A resposta em frequência do erro $G_{e}(s)$ pode ser encontrada calculando-se a diferença entre as respostas do modelo completo e reduzido:

$$
G_{e}(s)=G(s)-G_{r}(s)=\widetilde{C}(s) \cdot \Delta^{-1} \cdot \widetilde{B}(s)
$$

onde

$$
\begin{aligned}
& \Delta(s)=s I-A_{22}-A_{21}\left(s I-A_{11}\right)^{-1} A_{12} \\
& \widetilde{B}(s)=A_{21}\left(s I-A_{11}\right)^{-1} B_{1}+B_{2} \\
& \widetilde{C}(s)=C_{1}\left(s I-A_{11}\right)^{-1} A_{12}+C_{2}
\end{aligned}
$$

A dedução da equação acima é alcançada quando partimos das relações abaixo.

$$
\begin{aligned}
s I-A= & {\left[\begin{array}{cc}
I & 0 \\
-A_{21}\left(s I-A_{11}\right)^{-1} & I
\end{array}\right] } \\
& {\left[\begin{array}{cc}
\left(s I-A_{11}\right) & 0 \\
0 & \Delta(s)
\end{array}\right]\left[\begin{array}{cc}
I & -\left(s I-A_{11}\right)^{-1} A_{12} \\
0 & I
\end{array}\right] } \\
& {\left[\begin{array}{cc}
A_{11} & 0 \\
A_{21} & A_{22}
\end{array}\right]^{-1}=\left[\begin{array}{cc}
A_{11}^{-1} & 0 \\
-A_{22}^{-1} A_{21} A_{11}^{-1} & A_{22}^{-1}
\end{array}\right] } \\
& {\left[\begin{array}{cc}
A_{11} & A_{12} \\
0 & A_{22}
\end{array}\right]^{-1}=\left[\begin{array}{cc}
A_{11}^{-1} & -A_{11}^{-1} A_{12} A_{22}^{-1} \\
0 & A_{22}^{-1}
\end{array}\right] }
\end{aligned}
$$

Pode-se calcular o erro da resposta de estado estável em altas frequências aplicando o limite $\lim _{s \rightarrow \infty}$ à equação 133 . Com isso, pode-se escrever:

$$
\lim _{s \rightarrow \infty} G_{e}(s)=G_{e}(\infty)=0
$$

A equação 138 indica que o modelo reduzido por truncamento direto terá um bom comportamento em altas frequências. Entretanto o modelo reduzido apresenta erro na resposta estática $\lim _{s \rightarrow 0}\left[G_{e}(s)\right] \neq 0$, o que pode ser indesejado em muitas aplicações (OBINATA; ANDERSON, 2001) (MUSCATO, 2000). 


\subsection{ELIMINAÇÃO POR MÉTODOS DE PERTURBAÇÃO}

Os métodos de perturbação são usados em modelagem de ordem reduzida com a inclusão de parâmetros de perturbação, também chamados parâmetros singulares (KOKOTOVIC; KHALI; O'REILLY, 1987). Neste trabalho, serão usados parâmetros de perturbação que se propõem a desconsiderar o efeito transitório de estados mais rápidos de um sistema dinâmico (ERSAL et al., 2008) (SAMAR; POSTLETHWAITE; GU, 1994) (OBINATA; ANDERSON, 2001), este método também é chamado de análise de variedades lentas e rápidas (slow and fast manifold analysis).

Há muitas décadas, métodos de perturbação são amplamente usados na matemática. A literatura sobre o tema é vasta: (BENDER; ORSZAG, 1999), (NAYFEH, 2008), (HINCH, 1991), (LEVINSON, 1950) e suas aplicações são diversas. O autor destacou algumas aplicações de métodos de perturbação na redução da ordem de sistemas dinâmicos, descritos abaixo:

- Eliminação por perturbação singular (EPS) (ou singular perturbation approximation)

- Eliminação por perturbação singular generalizada (EPSG) (ou generalized singular perturbation approximation)

- Eliminação por perturbação singular generalizada e paramétrica (EPSGP) (ou parametric generalized singular perturbation approximation)

Para um sistema dinâmico apresentado na forma da equação 139 um termo de perturbação foi introduzido, separando as variáveis generalizadas em duas parcelas com dinâmicas diferentes.

$$
\begin{array}{lcr}
\dot{x_{1}}=f\left(x_{1}, x_{2}, \epsilon, t\right), & x_{1}\left(t_{0}\right)=x_{1}^{(0)}, & x_{1} \in \mathbb{R}^{n_{r}} \\
\epsilon \dot{x_{2}}=g\left(x_{1}, x_{2}, \epsilon, t\right), & x_{2}\left(t_{0}\right)=x_{2}^{(0)}, & x_{2} \in \mathbb{R}^{r}
\end{array}
$$

De acordo com Kokotovic (KOKOTOVIC; KHALI; O'REILLY, 1987), o objetivo desta estratégia de modelagem é representar a "resposta lenta" do sistema completo em outro de ordem reduzida (com a mesma ordem do vetor $x_{1}$ ). De acordo com o mesmo autor, a diferença entre o modelo de ordem reduzida e o completo está diretamente relacionada com a resposta transiente da parcela de "resposta rápida"do sistema. Portanto quanto mais rapidamente a parcela $x_{2}$, cuja velocidade é $\dot{x_{2}}=g / \epsilon$, convergir para o estado estável, menor será a discrepância entre a resposta do modelo reduzido em relação ao completo. 
O modelo reduzido será então representado pela equação 140 , onde $x_{r}$ é o vetor de GDL do sistema reduzido, cuja condição inicial $x_{1}^{0}$ é mantida.

$$
\dot{x_{r}}=f_{r}\left(x_{r}, \epsilon, t\right), \quad x_{r}\left(t_{0}\right)=x_{1}^{(0)}, \quad x_{r} \in \mathbf{R}^{n}
$$

A estratégia de adotar um parâmetro de perturbação para realizar a redução de um modelo, obtendo um outro de ordem reduzida, tem uma considerável desvantagem: nem sempre é fácil escolher parâmetros adequados, que ao serem considerados pequenos, reproduzem a resposta do modelo completo dentro de critérios aceitáveis.

Outro fator que deve ser considerado é a ordem do sistema reduzido. É esperado que quanto maior a diferença entre as ordens dos sistemas completo e reduzido, mais comprometida será a capacidade do sistema reduzido representar fidedignamente o completo. Este fator será tratado com maior profundidade no decorrer deste trabalho.

Para um sistema linear como apresentado pela equação 129 , pode-se assumir que existe uma relação do tipo $\dot{x_{2}}=\epsilon \cdot x_{2}$. Sendo assim, pode-se escrevê-lo na forma (KOKOTOVIC; KHALI; O'REILLY, 1987)

$$
\left\{\begin{array}{l}
\dot{x}_{r}=A_{11} x_{r}+A_{12} x_{2}+B_{1} u \\
\epsilon x_{2}=A_{21} x_{r}+A_{22} x_{2}+B_{2} u \\
y=C_{1} x_{r}+C_{2} x_{2}+D u
\end{array}\right.
$$

Nos itens abaixo, será mostrado que a escolha do parâmetro $\epsilon$ é determinante para o comportamento do sistema reduzido. O modelo de ordem reduzida é calculado isolando a componente $x_{2}$ na segunda linha da equação 141 e substituindo nas linhas 1 e 3 da mesma. Desta maneira, poderá ser escrito como na equação 142

$$
\left\{\begin{array}{c}
\dot{x}_{r} \\
\hline y_{r}
\end{array}\right\}=\left[\begin{array}{c|c}
A_{r} & B_{r} \\
\hline C_{r} & D_{r}
\end{array}\right] \cdot\left\{\begin{array}{c}
x_{r} \\
\hline u
\end{array}\right\}
$$

Como o vetor $x_{r}$ é uma aproximação de $x_{1}$, pode-se encontrar o vetor $x_{2 r}$ que é a aproximação do vetor $x_{2}$ que contém os graus de liberdade eliminados do sistema completo. Utilizando a equação 141 obtém-se a seguinte relação:

$$
x_{2 r}=\left(\epsilon I-A_{22}\right)^{-1}\left(A_{21} x_{r}+B_{2} u\right)
$$

\subsubsection{Eliminação por perturbação singular (EPS)}

No método de eliminação por perturbação singular (EPS), as variáveis menos significativas $x_{2}$ (selecionadas para serem eliminadas) são consideradas constantes $\dot{x}_{2}=0$ e consequente- 
mente e $\epsilon=0$.

Substituindo $\epsilon=0$ em 141, obtém-se:

$$
\left\{\begin{array}{l}
A_{r}=A_{11}-A_{12} A_{22}^{-1} A_{21} \\
B_{r}=B_{1}-A_{12} A_{22}^{-1} B_{2} \\
C_{r}=C_{1}-C_{2} A_{22}^{-1} A_{21} \\
D_{r}=D-C_{2} A_{22}^{-1} B_{2}
\end{array}\right.
$$

Onde o sistema reduzido formado pelas matrizes $A_{r}, B_{r}, C_{r}, D_{r}$ da equação 144 é definido na estrutura descrita na equação 142

Substituindo 144 em 4 e calculando a função de transferência do erro, conclui-se que a resposta estática do sistema reduzido seja igual ao do completo (SAMAR; POSTLETHWAITE; GU, 1994):

$$
\lim _{s \rightarrow 0}\left[G_{e}(s)\right]=0
$$

Pode-se verificar a conclusão acima calculando o erro da resposta estática do sistema reduzido, assumindo $\dot{x}=0$ e $\dot{x}_{r}=0$. Substituindo 144 em 24, obtém-se:

$$
\left[\begin{array}{ccc|c}
A_{11} & A_{12} & 0 & 0 \\
A_{21} & A_{22} & 0 & 0 \\
0 & 0 & A_{11}-A_{12} A_{22}^{-1} A_{21} & 0 \\
\hline C_{1} & C_{2} & -C_{1}+C_{2} A_{22}^{-1} A_{21} & -1
\end{array}\right]\left\{\begin{array}{c}
x_{1 e q} \\
x_{2 e q} \\
x_{r e q} \\
\hline e_{e q}
\end{array}\right\}=\left\{\begin{array}{c}
-B_{1} \\
-B_{2} \\
-B_{1}+A_{12} A_{22}^{-1} B_{2} \\
C_{2} A_{22}^{-1} B_{2}
\end{array}\right\} u_{d}
$$

Expandindo e simplificando 146, encontra-se $x_{r e q}=x_{1 e q}$ e consequentemente

$$
\left\|e_{e q}\right\|=0
$$

Este método de eliminação leva vantagem em aplicações onde é necessário que a resposta em estado estável para entrada degrau do sistema reduzido seja igual ao do completo. Um exemplo dessa necessidade é quando o projeto de um controlador que é baseado no modelo reduzido não admita erro de estado estável.

\subsubsection{Eliminação por perturbação singular generalizada (EPSG)}

No método de eliminação por perturbação singular generalizada (EPSG), considera-se uma relação de proporcionalidade entre os GDL do vetor $x_{2}$, que serão reduzidos, e suas respectivas 
derivadas. Desta maneira, $\dot{x}_{2}=\epsilon \cdot x_{2}$, sendo $\epsilon \in \mathbb{R}$

$$
\left\{\begin{array}{l}
A_{r}=A_{11}-A_{12}\left(\epsilon I-A_{22}\right)^{-1} A_{21} \\
B_{r}=B_{1}-A_{12}\left(\epsilon I-A_{22}\right)^{-1} B_{2} \\
C_{r}=C_{1}-C_{2}\left(\epsilon I-A_{22}\right)^{-1} A_{21} \\
D_{r}=D-C_{2}\left(\epsilon I-A_{22}\right)^{-1} B_{2}
\end{array}\right.
$$

Substituindo 148 em 4 e calculando a função de transferência do erro, conclui-se que $G(\epsilon)$ $G_{r}(\epsilon)=G_{e}(\epsilon)=0$. Portanto a resposta em frequência do sistema reduzido é igual à do completo quando submetidos a uma entrada senoidal de frequência $\epsilon$ (MUSCATO, 2000).

A relação $G(\epsilon)=G_{r}(\epsilon)$ pode ser encontrada ao substituir 148 em 18 e utilizar as seguintes relações:

$$
\left[\begin{array}{ll}
A_{11} & A_{12} \\
A_{21} & A_{22}
\end{array}\right]^{-1}=\left[\begin{array}{cc}
\hat{\Delta}^{-1} & -\hat{\Delta}^{-1} A_{12} A_{22}^{-1} \\
-A_{22}^{-1} A_{21} \hat{\Delta}^{-1} & A_{22}^{-1}+A_{22}^{-1} A_{21} \hat{\Delta}^{-1} A_{12} A_{22}^{-1}
\end{array}\right]
$$

com $\hat{\Delta}=A_{11}-A_{12} A_{22}^{-1} A_{21}$

e

$$
\left(A_{11}-A_{12} A_{22}^{-1} A_{21}\right)^{-1}=A_{11}^{-1}+A_{11}^{-1} A_{12}\left(A_{22}-A_{21} A_{11}^{-1} A_{12}\right)^{-1} A_{21} A_{11}^{-1}
$$

Considerando que $A_{11}$ e $A_{22}$ são matrizes com determinante não nulo.

\subsubsection{Eliminação por perturbação singular generalizada e paramétrica (EPSGP)}

No método de eliminação por perturbação singular generalizada e paramétrica (EPSGP), considera-se que existam diferentes relações de proporcionalidade para cada GDL que compõe o vetor $x_{2} . \dot{x}_{2}=P \cdot x_{2}$ sendo $P$ uma matriz diagonal que pondera separadamente cada um dos GDL que serão reduzidas do sistema completo.

$$
\begin{aligned}
& \left\{\begin{array}{c}
\dot{x}_{21} \\
\dot{x}_{22} \\
\vdots \\
\dot{x}_{2 n}
\end{array}\right\}=[P] \cdot\left\{\begin{array}{c}
x_{21} \\
x_{22} \\
\vdots \\
x_{2 n}
\end{array}\right\}=\left[\begin{array}{cccc}
\epsilon_{1} & 0 & 0 & 0 \\
& \epsilon_{2} & 0 & 0 \\
& & \ddots & 0 \\
& & & \epsilon_{2 n}
\end{array}\right] \cdot\left\{\begin{array}{c}
x_{21} \\
x_{22} \\
\vdots \\
x_{2 n}
\end{array}\right\} \\
& \left\{\begin{array}{l}
A_{r}=A_{11}-A_{12}\left(P-A_{22}\right)^{-1} A_{21} \\
B_{r}=B_{1}-A_{12}\left(P-A_{22}\right)^{-1} B_{2} \\
C_{r}=C_{1}-C_{2}\left(P-A_{22}\right)^{-1} A_{21} \\
D_{r}=D-C_{2}\left(P-A_{22}\right)^{-1} B_{2}
\end{array}\right.
\end{aligned}
$$


Uma vantagem do método EPSGP é a liberdade do analista realizar a sintonização dos parâmetros $\epsilon_{i}$ de $P$ de acordo com sua necessidade. 


\section{CORREÇÕES DE MODELOS REDUZIDOS}

Em alguns casos, a base e o método de eliminação escolhidos não satisfazem completamente os requisitos especificados para o modelo reduzido (MARSHALL, 1966), (DAVISON, 1967), (FOSSARD, 1970), (VARGA, 1993). Porém, esta escolha foi necessária para garantir outros comportamentos também desejados no modelo reduzido, como preservação da estabilidade, controlabilidade, etc.

Após a eliminação das variáveis do sistema completo, ainda fica a critério do analista realizar correções no sistema reduzido. Cabe lembrar que esta etapa não é obrigatória, porém é desejável caso o analista queira melhorar a representatividade do modelo reduzido realizando correções nos seguintes parâmetros:

a ) Resposta em uma frequência determinada $G_{r}\left(s_{0}\right)$

b ) Resposta estática do sistemas

c ) Sobre-sinal para entrada degrau

Como contribuição do autor neste trabalho, foram propostas nos itens 8.2 e 8.3 novas maneiras de, após a eliminação das variáveis selecionadas, realizar correções no modelo reduzido.

\subsection{RESPOSTA EM UMA FREQUÊNCIA DETERMINADA}

Através de modificações nas matrizes do sistema reduzido, pode-se corrigí-lo para que o valor da função de transferência do erro seja nulo em uma frequência $s_{0}$ determinada. Nos items 8.1.1 e 8.1.2 serão apresentados dois métodos que alteram a resposta do sistema reduzido através da modificação das matrizes $D_{r}$ e $C_{r}$ respectivamente.

\subsubsection{Correção de $G_{r}\left(s_{0}\right)$ através de $D_{r}$}

No método de correção através da matriz $D_{r}$, deseja-se encontrar uma matriz corrigida $D_{r c}$ que substitua $D_{r}$ no sistema reduzido (VARGA, 1993). O sistema reduzido e corrigido será composto pelas matrizes $\left(A_{r}, B_{r}, C_{r}, D_{r c}\right)$.

A função de transferência do erro do modelo reduzido (avaliada em uma frequência $s_{0}$ ) é:

$$
G_{e}\left(s_{0}\right)=G\left(s_{0}\right)-G_{r}\left(s_{0}\right)=C\left(s_{0} I-A\right)^{-1} B+D-C_{r}\left(s_{0} I-A_{r}\right)^{-1} B_{r}-D_{r}
$$


Realizando a correção para que $G_{e}\left(s_{0}\right)=0$, ou seja $G\left(s_{0}\right)=G_{r c}\left(s_{0}\right)$, obtém-se a matriz corrigida $D_{r c}$ :

$$
D_{r c}=C\left(s_{0} I-A\right)^{-1} B+D-C_{r}\left(s_{0} I-A_{r}\right)^{-1} B_{r}
$$

Cabe lembrar que em alguns métodos de redução, a função de transferência do erro para frequências muito altas tende a ser nula $G_{e}(\infty)=0 \mathrm{e}$, com a utilização deste método de correção, o valor de $G_{e}(\infty)$ pode ser não nulo, o que é indesejável em algumas aplicações.

\subsubsection{Correção de $G_{r}\left(s_{0}\right)$ através de $C_{r}$}

No método de correção através da matriz $C_{r}$, o sistema reduzido corrigido é composto pelas matrizes $\left(A_{r}, B_{r}, C_{r c}, D_{r}\right)$ (VARGA, 1993). Sendo que a matriz corrigida $C_{r c}$ é calculada segundo a equação 155 .

$$
C_{r c}=C_{r}+C_{2} E
$$

A função de transferência do modelo reduzido corrigido é:

$$
G_{r c}\left(s_{0}\right)=C_{r}\left(s_{0} I-A_{r}\right)^{-1} B_{r}-C_{2} E\left(s_{0} I-A_{r}\right)^{-1} B_{r}+D_{r}=G\left(s_{0}\right)
$$

Desta maneira, pode-se sintonizar os valores dos componentes da matriz $E$ tal que $G_{e}\left(s_{0}\right)=$ 0 .

Como a matriz $G_{e}$ tem $n_{s} n_{e}$ termos, a matriz $E$ deve ter no mínimo a mesma quantidade de termos para que e equação 156 sempre tenha solução. Como a matriz $E$ tem $n_{r}^{2}$ termos, a condição necessária para que 156 sempre tenha solução é

$$
n_{r}^{2} \geq n_{s} n_{e}
$$

O que é normalmente satisfeito para a maioria das aplicações (VARGA, 1993).

A quantidade de parâmetros para serem sintonizados na matriz $\mathrm{E}$ pode ser maior que $n_{s} n_{e}$, neste caso a equação 156 terá infinitas soluções. Desta maneira, a sintonização dos termos da matriz $E$ pode ser utilizada para realizar otimizações, como por exemplo minimizar normas do erro $\left\|G_{e}\right\|_{\mathcal{H}_{2}}$ ou $\left\|G_{e}\right\|_{\mathcal{H}_{\infty}}$.

Uma vantagem da utilização deste método de correção é que a matriz $D_{r}$ não é alterada como no item 8.1 .1 fazendo que o valor de $G_{e}(\infty)$ seja preservado após a correção. 


\subsection{CORREÇÃO DA RESPOSTA ESTÁTICA}

Um método para garantir que resposta estática seja preservada no sistema reduzido é, após a redução, avaliar a relação entre as respostas de estado estável para entrada degrau dos sistemas completo e reduzido.

Como contribuição deste trabalho, o autor propõe que o sistema reduzido corrigido seja composto por $\left(A_{r}, B_{r}, E C_{r}, D_{r}\right)$. Sendo que $E$ é uma matriz diagonal e $C_{r c}=E C_{r}$. Neste caso, a resposta estática de ambos sistemas será calculada através da equação 23 e será considerado $u \in \mathbb{R}^{1}$.

A partir da equação 23, os vetores $y_{e q}$ e $y_{\text {req }}$ que representam a resposta de equilíbrio para entrada degrau dos sistemas completo e reduzido respectivamente, podem ser escritos na forma

$$
\begin{aligned}
& y_{e q}=\left(y_{e q 1}, y_{e q 2}, \ldots, y_{e q n}\right)^{T} \\
& y_{r e q}=\left(y_{r e q 1}, y_{r e q 2}, \ldots, y_{\text {reqn }}\right)^{T}
\end{aligned}
$$

De forma análoga, o estado de cada sistema é respectivamente.

$$
\begin{aligned}
& x_{e q}=\left(x_{e q 1}, x_{e q 2}, \ldots, x_{e q n}\right)^{T} \\
& x_{r e q}=\left(x_{r e q 1}, x_{r e q 2}, \ldots, x_{r e q n}\right)^{T}
\end{aligned}
$$

Também sabe-se que

$$
y_{e q i}=C(i,:) x_{e q i}
$$

onde $C(i,:)$ representa a i-ésima linha da matriz $\mathrm{C}$ e $y_{\text {eqi }}$ e $x_{e q i}$ o i-ésimo valor dos vetores $y_{e q}$ e $x_{e q}$ respectivamente.

Sendo assim, aplica-se um fator de correção $f_{e q i}$ em cada i-ésima linha na matriz $C_{r}$ do sistema reduzido tal que

$$
f_{\text {eqi }}=\frac{y_{\text {eqi }}}{y_{\text {reqi }}}
$$

e

$$
y_{r c e q i}=f_{e q i} C_{r}(i,:) x_{r e q i}=C_{r c}(i,:) x_{r e q i}=y_{e q i}
$$

Calcula-se a matriz $C_{r c}$ do sistema reduzido corrigido multiplicando-se uma matriz com os 
fatores de correção na diagonal principal por $C_{r}$

$$
C_{r c}=E C_{r}=\left[\begin{array}{lll}
f_{e q 1} & & \\
& \ddots & \\
& & f_{\text {eqm }}
\end{array}\right] C_{r}
$$

onde $C_{r c}$ é uma matriz corrigida para que o erro de estado estável para entrada degrau seja nulo, ou seja, $C x_{e q}=C_{r c} x_{r e q}$.

A figura 11 mostra um exemplo da correção de um sistema reduzido $\left(A_{r}, B_{r}, C_{r}, D_{r}\right)$. $\mathrm{Na}$ figura, a resposta $y_{r}(t)$ do sistema reduzido apresenta erro estático, enquanto que a resposta $y_{r c}(t)$ do sistema reduzido e corrigido $\left(A_{r}, B_{r}, C_{r c}, D_{r}\right)$ não possui erro estático.

Figura 11 - Exemplo de correção para resposta estática.

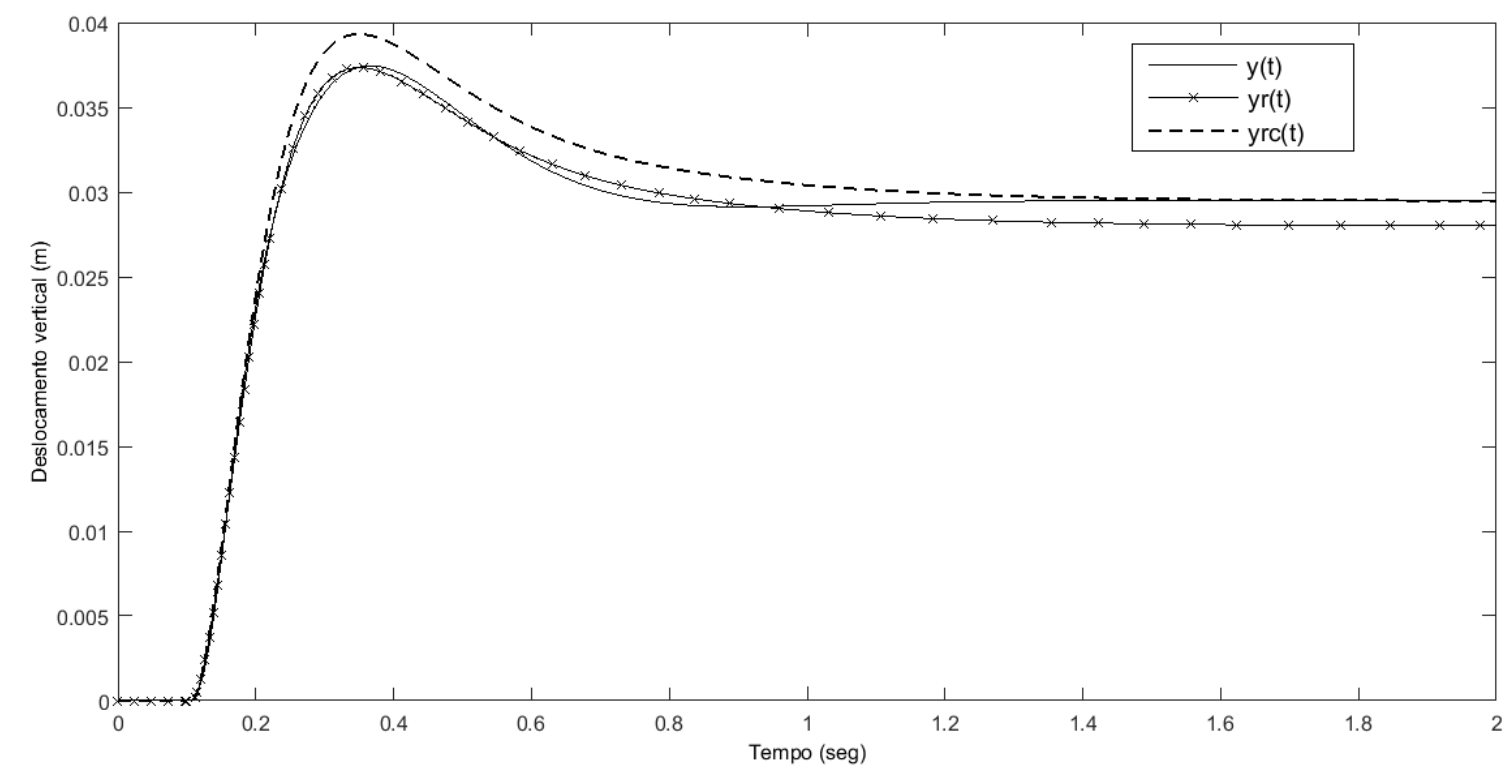

No método de correção da resposta estática, as respostas estáticas dos modelos completo e reduzido não corrigido devem ser calculadas. Isso pode ser uma desvantagem devido ao custo computacional envolvido.

Cabe lembrar que o método apresentado neste item apenas aplica fatores de escala na matriz $C_{r}$ da equação 3 , o que não influencia a estabilidade e na controlabilidade do sistema reduzido. A partir da equação 15, pode-se concluir que o mesmo não ocorre com a observabilidade do sistema reduzido, que é influenciada após a implementação dos métodos de correção abaixo e deve ser verificada posteriormente.

Um exemplo numérico da correção da resposta estática de um SR pode ser encontrada em anexo no item C.4. 


\subsection{CORREÇÃO DO MÁXIMO SOBRE-SINAL}

De maneira análoga à resposta estática, o sobre-sinal máximo também pode ser corrigido no sistema reduzido através da aplicação de um fator de correção para cada linha da matriz $C_{r}$ do modelo de ordem reduzida.

Como contribuição deste trabalho, o autor propõe que o sistema reduzido corrigido seja composto por $\left(A_{r}, B_{r}, C_{r c}, D_{r}\right)$. Sendo que a matriz $C_{r c}$ é calculada segundo a equação 161 .

$$
C_{r c}=E C_{r}
$$

A matriz $E$ é uma matriz diagonal cujos valores de sua diagonal principal são os fatores de correção utilizados para realizar a correção do máximo sobre-sinal do SR. Para o método de correção do máximo sobre-sinal, será considerado que o vetor de entrada seja de ordem 1 , ou seja, $u \in \mathbb{R}^{1}$.

O máximo sobre-sinal da saída $y_{i}(t)$ para uma entrada degrau $u_{d}$ é:

$$
y_{s s i}=\frac{\left\|y_{i}(t)\right\|_{\mathcal{L}_{\infty}}-y_{e q i}}{y_{\text {eqi }}}
$$

Onde a norma $\left\|y_{i}(t)\right\|_{\mathcal{L}_{\infty}}$ é igual ao máximo valor de $y_{i}(t)$.

O fator de correção para o máximo sobre-sinal é calculado através da razão entre as normas $\mathcal{L}_{\infty}$ das respostas para entrada degrau:

$$
f_{s s i}=\frac{\left\|y_{i}(t)\right\|_{\mathcal{L}_{\infty}}}{\left\|y_{r i}(t)\right\|_{\mathcal{L}_{\infty}}}
$$

De maneira análoga ao item 8.2. calcula-se $C_{r c}$ que é a matriz corrigida para que máximo sobre-sinal seja igual nos sistemas completo e reduzido, ou seja, $\left\|y_{i}(t)\right\|_{\mathcal{L}_{\infty}}=\left\|y_{r i}(t)\right\|_{\mathcal{L}_{\infty}}$

$$
C_{r c}=E C_{r}=\left[\begin{array}{ccc}
f_{s s 1} & & 0 \\
& \ddots & \\
0 & & f_{s s m}
\end{array}\right] C_{r}
$$

A figura 12 mostra um exemplo de modelo reduzido cujo erro de máximo sobre-sinal foi corrigido para zero.

Cabe lembrar que o método apresentado neste item apenas aplica fatores de escala na matriz $C_{r}$ da equação 3, o que não influencia a estabilidade e na controlabilidade do sistema reduzido. A partir da equação 15, pode-se concluir que o mesmo não ocorre com a observabilidade do sistema reduzido, que é influenciada após a implementação dos métodos de correção abaixo e deve ser verificada posteriormente. 
Figura 12 - Exemplo de correção para máximo sobre-sinal.

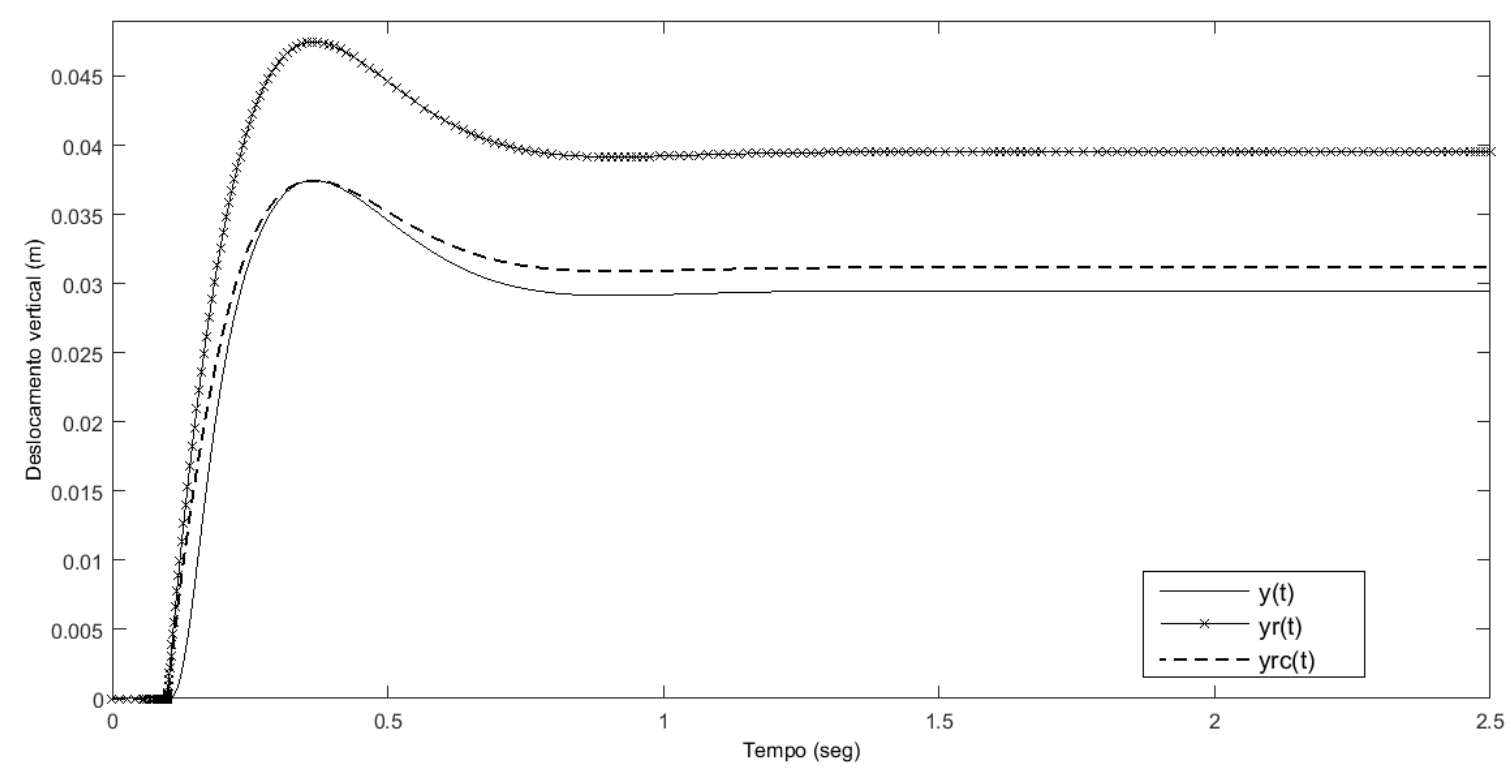

Pode-se perceber que, através dos métodos propostos, a correção do sistema reduzido para anular o erro da resposta estática é contraditória com a anulação do erro de sobre-sinal máximo. Portanto o analista deve levar em conta que ao corrigir a resposta estática, o máximo sobre-sinal será diretamente influenciado e vice versa.

Se os erros para resposta estática e para sobre-sinal forem simultaneamente positivos (ou negativos), ao realizar a correção do menor erro em módulo, o outro erro será diminuído (porém não anulado). Pode-se notar que este caso está representado no exemplo da figura 12 Entretanto, se os erros tiverem sinais opostos, ao se corrigir o erro da resposta estática, o erro do sobre-sinal máximo aumentará em módulo (e vice-versa). 


\section{REPRESENTATIVIDADE DE MODELOS REDUZIDOS}

Neste capítulo, são apresentadas maneiras de comparar o comportamento de modelos reduzidos dinâmicos lineares. Portanto serão apresentadas métricas com o objetivo de quantificar a capacidade que um SR tem de reproduzir as características dinâmicas do SC.

As métricas aqui apresentadas traduzem características dinâmicas dos modelos completo e reduzido (ou do erro entre os mesmos) em valores escalares. Desta maneira, a comparação entre diferentes metodologias de redução se torna mais simples e objetiva pois é feita através da comparação entre valores escalares.

Ao final do capítulo, o autor propõe uma metodologia de calcular a representatividade total de um SR.

\subsection{MÉTRICAS DA RESPOSTA NO DOMÍNIO DO TEMPO}

Serão apresentadas algumas métricas baseadas em características da resposta no domínio do tempo de um SR.

Conforme apresentado na equação 4 o erro $e(t)$ de um SR é a diferença entre a resposta dinâmica dos sistemas completo e reduzido $e(t)=y(t)-y_{r}(t)$ e pode ser calculado através do sistema

$$
\left[\begin{array}{cc|c}
A & 0 & B \\
0 & A_{r} & B_{r} \\
\hline C & -C_{r} & D-D_{r}
\end{array}\right]\left\{\begin{array}{c}
x \\
x_{r} \\
\hline u
\end{array}\right\}=\left\{\begin{array}{c}
\dot{x} \\
\dot{x}_{r} \\
\hline e
\end{array}\right\}
$$

\subsubsection{Erro quadrático}

O erro quadrático $e_{q}(t)$ é uma maneira de contabilizar em uma única curva a resposta temporal o vetor $e(t)=\left[e_{1}(t) ; e_{2}(t) ; \ldots ; e_{n_{y}}(t)\right)^{T}$ do $\mathrm{SR}$, o que falicita a avaliação do comportamento do modelo.

$$
e_{q}(t)=\sqrt{e_{1}^{2}(t)+e_{2}^{2}(t)+\cdots+e_{n_{y}}^{2}(t)}=\sqrt{e^{T}(t) e(t)}
$$

Como as componentes $y_{i}(t)$ de $y(t)=\left[y_{1}(t) ; \ldots ; y_{n_{y}}(t)\right]^{T}$ podem ter ordens de grandeza diferentes, o erro quadrático calculado na equação 165 poderá ser predominentemente influenciado por algumas componentes do vetor de resposta que possuem ordem de grandeza maior. Sendo assim, o autor propõe uma ponderação para cada componente do vetor de resposta 
através de uma matriz de ponderação $P$. O erro quadrático ponderado $e_{q p}(t)$ é portanto

$$
e_{q p}(t)=\sqrt{p_{1} e_{1}^{2}(t)+p_{2} e_{2}^{2}(t)+\cdots+p_{n_{y}} e_{n_{y}}^{2}(t)}=\sqrt{e^{T}(t) P e(t)}
$$

onde $P=\operatorname{diag}\left(p_{1}, p_{2}, \ldots, p_{n_{y}}\right)$ e as componentes $p_{i}$ são valores constantes. As equações 166 e 165 são equivalentes quando a matriz $P$ é uma matriz identidade.

\subsubsection{Norma $\mathcal{L}_{2}$ do erro}

Uma métrica de comparação baseada no domínio do tempo é a norma $\mathcal{L}_{2}$ do erro $e(t)$. Diferente do erro quadrático, a norma $\mathcal{L}_{2}$ do erro quantifica a diferença entre as respostas dos sistemas completo e reduzido em apenas um valor escalar. Isso possibilita que a comparação entre modelos reduzidos seja mais simples e objetiva através da comparação entre valores escalares.

Vale lembrar que uma propriedade das normas usadas neste trabalho é que todas possuem valores reais e não negativos. Uma descrição mais detalhada sobre esta e outras normas é encontrada no Apêndice $B$.

A norma $\mathcal{L}_{2}$ do erro $e(t)$ é a raiz quadrada da integral do erro quadrático no intervalo $\left[t_{0}, t_{f}\right]$.

$$
\left\|y(t)-y_{r}(t)\right\|_{\mathcal{L}_{2}\left[t_{0}, t_{f}\right]}=\|e(t)\|_{\mathcal{L}_{2}\left[t_{0}, t_{f}\right]}=\left(\int_{t_{0}}^{t_{f}} e^{T}(\tau) e(\tau) d \tau\right)^{\frac{1}{2}}
$$

Quanto menor o valor da norma $\mathcal{L}_{2}$ do erro, melhor é a aproximação do modelo reduzido naquele intervalo, sendo que quando $\left\|y(t)-y_{r}(t)\right\|_{\mathcal{L}_{2}\left[t_{0}, t_{f}\right]}=0$, a resposta $y_{r}(t)$ do sistema é idêntica à do modelo completo em todo o intervalo $t \in\left[t_{0}, t_{f}\right]$.

Uma desvantagem sobre a utilização da métrica mostrada na equação 167 é a necessidade de se realizar uma simulação dinâmica transiente para que a norma seja calculada. Além disso, valor encontrado não é uma característica que depende somente da natureza do sistema, mas também depende da excitação de entrada aplicada.

Em algumas aplicações, não é possível reproduzir a mesma excitação de entrada em dois sistemas distintos. Nestes casos, diferenças entre as respostas dos sistemas comparados podem ser oriundas não apenas do erro causado pelo processo de redução da ordem do modelo, mas também pela diferença entre as excitações de entrada. Desta maneira, outras métricas que diminuem a influência causada pela excitação de entrada podem ser mais adequadas.

A métrica $\mathcal{M}_{1}$ é uma alternativa para diminuir a influência do sinal de entrada na métrica de comparação. Ela é calculada através da razão entre a norma $\left\|y(t)-y_{r}(t)\right\|_{\mathcal{L}_{2}\left[t_{0}, t_{f}\right]}$ e a 
norma $\mathcal{L}_{2}$ do sinal de entrada $u(t)$.

$$
\mathcal{M}_{1}=\frac{\|e(t)\|_{\mathcal{L}_{2}\left[t_{0}, t_{f}\right]}}{\|u(t)\|_{\mathcal{L}_{2}\left[t_{0}, t_{f}\right]}}=\frac{\left(\int_{0}^{t} e^{T}(\tau) e(\tau) d \tau\right)^{\frac{1}{2}}}{\left(\int_{0}^{t} u^{T}(\tau) u(\tau) d \tau\right)^{\frac{1}{2}}}
$$

Outra alternativa para diminuir a influência do sinal de entrada na métrica de comparação é através da métrica $\mathcal{M}_{2}$, descrita na equação 169 (ANTOULAS, 2005)

$$
\mathcal{M}_{2}=\sup _{u \neq 0} \frac{\left[\int_{0}^{\infty} e^{T}(t) e(t) d t\right]^{\frac{1}{2}}}{\int_{0}^{\infty} \sqrt{u_{1}^{2}(t)+\cdots+u_{m}^{2}(t)} d t}=\left[\lambda_{\max }\left(B^{*} L_{o} B\right)\right]^{\frac{1}{2}}
$$

Uma vantagem de utilizar a métrica $\mathcal{M}_{2}$ é que não há necessidade de realizar uma simulação dinâmica transiente para calculá-la.

Uma outra desvantagem de utilizar a norma $\|e(t)\|_{\mathcal{L}_{2}\left[t_{0}, t_{f}\right]}$ é que ela traz apenas informações do erro longo do tempo. Com isso, o analista não saberá se os valores do erro $e(t)$ são pequenos em relação aos valores da resposta $y(t)$ do SC. Portanto o autor propõe uma métrica que é a razão entre as normas $\mathcal{L}_{2}$ do erro e da resposta $y(t)$ do $\mathrm{SC}$ no intervalo $\left[t_{0}, t_{f}\right]$.

$$
\mathcal{M}_{\mathcal{L}_{2}}=\frac{\|e(t)\|_{\mathcal{L}_{2}\left[t_{0}, t_{f}\right]}}{\|y(t)\|_{\mathcal{L}_{2}\left[t_{0}, t_{f}\right]}}
$$

Esta métrica quantifica a capacidade que o modelo reduzido tem de reproduzir a resposta $y(t)$ do modelo completo no intervalo $t \in\left[t_{0}, t_{f}\right]$. Desta maneira, quando $\mathcal{M}_{\mathcal{L}_{2}}$ possuir valor nulo, a resposta $y_{r}(t)$ do SR é idêntica à do $\mathrm{SC}$ em todo o intervalo $\left[t_{0}, t_{f}\right]$.

Como exemplo, supondo que a resposta do modelo reduzido seja $y_{r}(t)=2 y(t)$ (neste caso o erro é de $100 \%$ em relação ao modelo completo), substituindo esta condição na equação 175, encontra-se $\mathcal{M}_{\mathcal{L}_{2}}=1$. Portanto o valor de $\mathcal{M}_{\mathcal{L}_{2}}=1$ corresponde a um erro médio de $100 \%$ de $y_{r}(t)$ em relação a $y(t)$.

\subsubsection{Máximo sobre-sinal}

Para um sistema subamortecido submetido a uma entrada degrau, o máximo sobre-sinal é igual ao o valor máximo da curva de resposta $y_{i}(t)$, medido a partir da condição de equilíbrio $y_{e q i}$, normalizado pelo valor de equilíbrio. (OGATA, 2001)

Como se pode ver na figura 13 , o máximo valor da curva de resposta $y_{i}(t)$ é igual à norma 
$\left\|y_{i}(t)\right\|_{\mathcal{L}_{\infty}}$, portanto pode-se escrever o máximo sobre-sinal $y_{s i}$ como:

$$
y_{s i}=\frac{\left\|y_{i}(t)\right\|_{\mathcal{L}_{\infty}}-y_{e q i}}{y_{\text {eqi }}}
$$

Figura 13 - Exemplo gráfico do sobre-sinal.

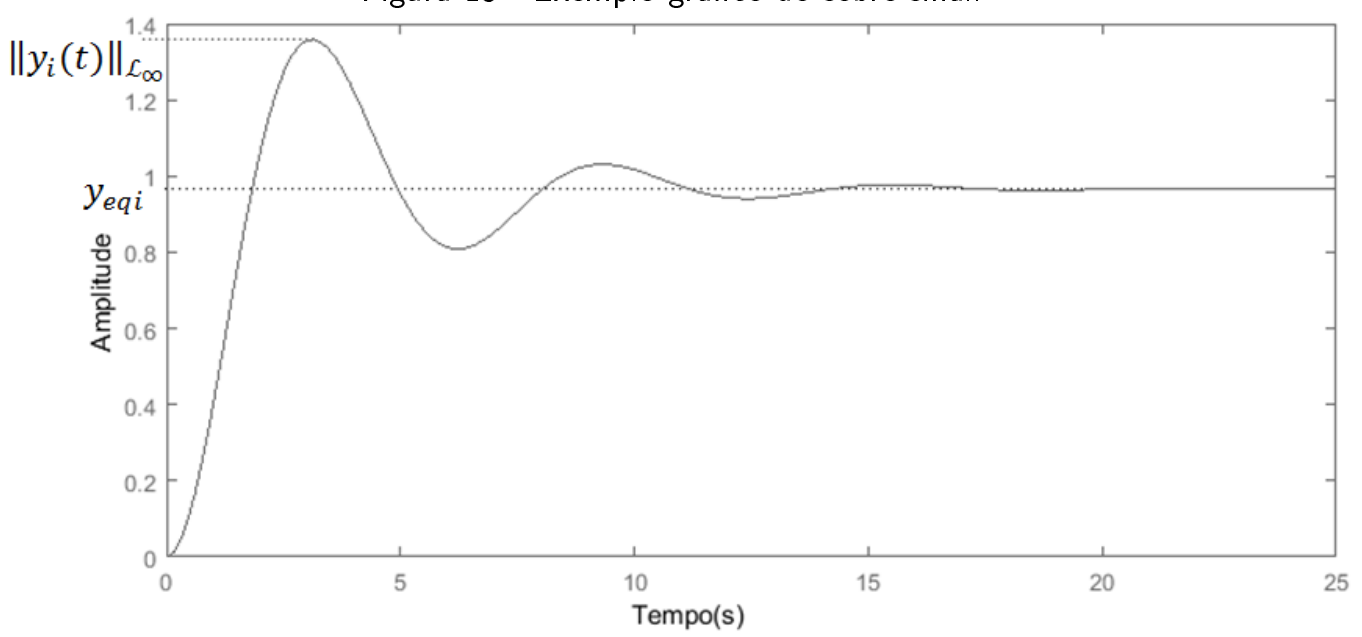

Para modelos cuja ordem $n_{y}$ do vetor de saidas $y(t)$ é elevada, a avaliação da capacidade que um SR tem de reproduzir os sobre-sinais do SC se torna dificultosa pela quantidade de valores a serem comparados.

Portanto como contribuição neste trabalho, o autor propõe uma métrica $\mathcal{M}_{s s}$ que quantifica em um valor escalar a capacidade que o modelo reduzido tem para reproduzir os sobre-sinais do SC.

A métrica $\mathcal{M}_{s s}$ é igual à raiz da soma dos quadrados dos erros dos sobre-sinais do modelo reduzido:

$$
\mathcal{M}_{s s}=\left[\sum_{i=1}^{n_{y}}\left(\frac{y_{s i}-y_{r s i}}{y_{s i}}\right)^{2}\right]^{\frac{1}{2}}
$$

Onde $y_{s i}$ e $y_{r s i}$ são os sobre-sinais da i-ésima saída dos sistemas completo e reduzido respectivamente, calculados para uma mesma entrada degrau $u_{d}$.

Quando a métrica $\mathcal{M}_{s s}$ possuir valor nulo, os sobre-sinais $y_{r s i}$ de todas as componentes do vetor de saída do modelo reduzido são idênticos aos do modelo completo.

\subsubsection{Tempo de acomodação}

Para sistemas subamortecidos ou sobreamortecidos, submetidos a uma entrada degrau, o tempo de acomodação $t_{s}$ é o tempo necessário para que a curva de resposta $y_{i}(t)$ alcance 
valores em uma faixa (usualmente de $2 \%$ ou $5 \%$ ) em torno do valor final $y_{\text {eq }}$, nela permanecendo indefinidamente (OGATA, 2001). Neste trabalho, o tempo de acomodação será correspondente a $2 \%$ de tolerância em torno do valor de equilíbrio.

A figura 14 mostra uma representação gráfica do tempo de acomodação.

Figura 14 - Exemplo gráfico do tempo de acomodação.

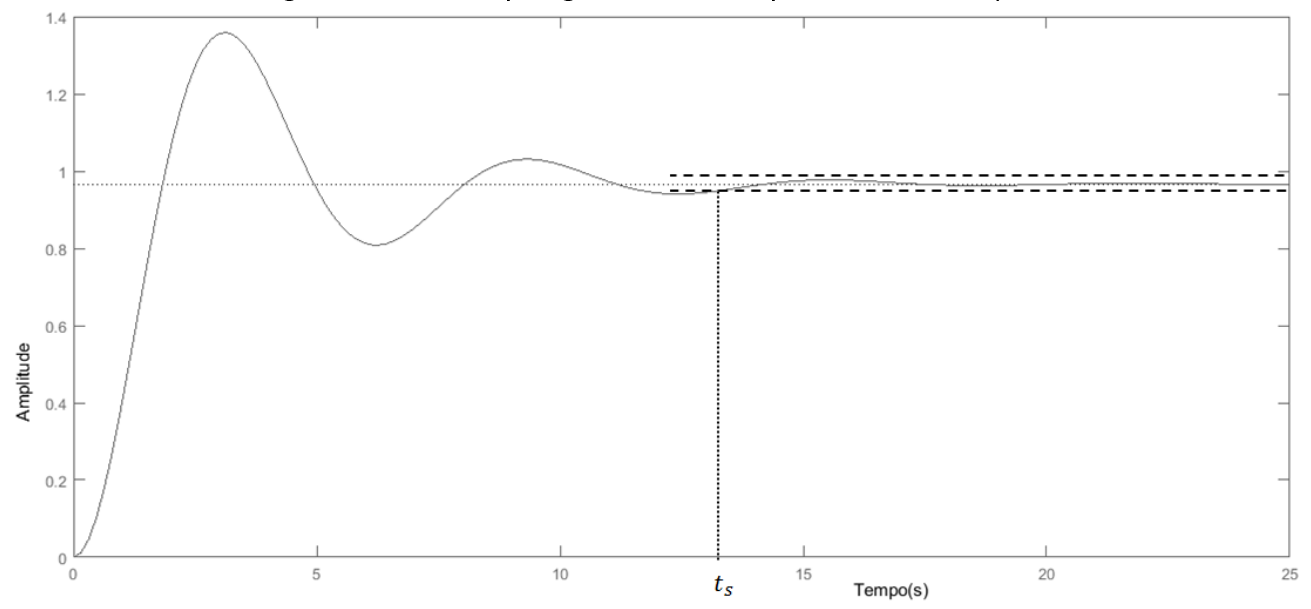

Entretanto, para modelos cuja ordem $n_{y}$ do vetor de saidas $y(t)$ é elevada, a avaliação da capacidade que um SR tem de reproduzir os tempos de acomodação do SC se torna dificultosa pela quantidade de valores a serem comparados.

Portanto como contribuição neste trabalho, o autor propõe uma métrica $\mathcal{M}_{s}$ que quantifica em um valor escalar a capacidade que o modelo reduzido tem para reproduzir os tempos de acomodação do SC.

A métrica $\mathcal{M}_{s}$ é igual à raiz da soma dos quadrados dos erros dos tempos de acomodação do modelo reduzido:

$$
\mathcal{M}_{s}=\left[\sum_{i=1}^{n_{y}}\left(\frac{t_{s i}-t_{r s i}}{t_{s i}}\right)^{2}\right]^{\frac{1}{2}}
$$

Onde $t_{s i}$ e $t_{r s i}$ são os tempos de acomodação da i-ésima saída dos sistemas completo e reduzido respectivamente.

Quando a métrica $\mathcal{M}_{s}$ possuir valor nulo, os tempos de acomodação $t_{r s i}$ de todas as componentes do vetor de saída do modelo reduzido são idênticos aos do modelo completo.

\subsection{MÉTRICAS DA RESPOSTA NO DOMÍNIO DA FREQUÊNCIA}

Nos itens 9.2.1 a 9.2.3 serão apresentadas duas métricas baseadas em características da resposta no domínio da frequência de um modelo reduzido. 


\subsubsection{Norma $\mathcal{H}_{2}$ da função de transferência}

Neste trabalho, a norma $\mathcal{H}_{2}$ será usada para avaliar comportamentos de funções de transferência de modelos.

A norma $\mathcal{H}_{2}$ de uma função de transferência $G(s=i \omega)$ quantifica os valores das amplitudes da mesma no intervalo $\omega \in]-\infty, \infty$ [ e é calculada segundo a equação 174 .

$$
\|G(s)\|_{\mathcal{H}_{2}}=\left(\frac{1}{2 \pi} \int_{-\infty}^{\infty} \operatorname{tr}\left[G^{*}(i \omega) G(i \omega)\right] d \omega\right)^{\frac{1}{2}}
$$

Assumindo que $\mathrm{D}=0$ na equação 2 e que o sistema seja assintoticamente estável, as seguintes normas da função de transferência $G(s)$ e da resposta ao impulso $g(t)$ são numericamente iguais: $\|G(s)\|_{\mathcal{H}_{2}}=\|G(s)\|_{\mathcal{L}_{2}}=\|g(t)\|_{\mathcal{L}_{2}}$ (ANTOULAS, 2005).

Como consequência, obtém-se:

$$
\|G(s)\|_{\mathcal{H}_{2}}=\|g(t)\|_{\mathcal{L}_{2}}=\left(\int_{0}^{\infty} \operatorname{tr}\left[g^{*}(t) g(t)\right] d t\right)^{\frac{1}{2}}
$$

Usando a solução do sistema para entrada impulsiva, temos:

$$
g(t)= \begin{cases}C e^{A t} B+\delta D & t \geq 0 \\ 0 & t<0\end{cases}
$$

Substituindo 176 em 175 e sabendo que $\operatorname{tr}\left[g^{*}(t) g(t)\right]=\operatorname{tr}\left[g(t) g^{*}(t)\right]$, chega-se às relações:

$$
\|G(s)\|_{\mathcal{H}_{2}}=\|g(t)\|_{\mathcal{L}_{2}}=\sqrt{\operatorname{tr}\left[B^{*} L_{o} B\right]}=\sqrt{\operatorname{tr}\left[C L_{c} C^{*}\right]}=\sqrt{\operatorname{tr}\left[C L_{x} B\right]}
$$

O uso da equação 177 é vantajoso em relação à equação 174 pois não existe a necessidade de se calcular a integral da equação 174.

O uso da equação 177 é vantajoso pois fornece o valor exato da integral da equação 174.

Caso seja de preferência do analista, pode-se calcular um limite superior (que não é mínimo) para a norma $\|G\|_{\mathcal{H}_{2}}$ através da relação 178 (NARASIMHAMURTHI, 1987)

$$
\|G(s)\|_{\mathcal{H}_{2}} \leq \sqrt{2 \operatorname{tr}(-A)}\|G\|_{\mathcal{H}_{\infty}}
$$

O autor propõe uma métrica $\mathcal{M}_{\mathcal{H}_{2}}$ que é a razão entre as normas $\mathcal{H}_{2}$ da função de transferência $G_{e}(s)$ do erro do modelo reduzido e da função de transferência $G(s)$ do modelo completo.

$$
\mathcal{M}_{\mathcal{H}_{2}}=\frac{\left\|G_{e}\right\|_{\mathcal{H}_{2}}}{\|G\|_{\mathcal{H}_{2}}}
$$

Desta maneira, quando $\mathcal{M}_{\mathcal{H}_{2}}$ possuir valor nulo, a função de transferência do modelo reduzido é idêntica à do modelo completo no intervalo $\omega \in]-\infty, \infty[$. 
Como exemplo, supondo que a função de transferência do modelo reduzido seja $G_{r}(s)=$ $2 G(s)$ (neste caso o erro é de $100 \%$ em relação ao modelo completo), substituindo esta condição na equação 179 encontra-se $\mathcal{M}_{\mathcal{H}_{2}}=1$. Portanto o valor de $\mathcal{M}_{\mathcal{H}_{2}}=1$ corresponde a um erro médio de $100 \%$ de $G_{r}(s)$ em relação a $G(s)$.

\subsubsection{Norma $\mathcal{H}_{\infty}$ da função de transferência}

A norma $\mathcal{H}_{\infty}$ de uma função de transferência também pode ser usada como métrica para a comparação entre sistemas dinâmicos. Ela é igual ao maior valor que o máximo valor singular da função de transferência $G(s=i \omega)$ pode ter no intervalo $\omega \in[0, \infty[$.

$$
\|G\|_{\mathcal{H}_{\infty}}=\sup _{\omega \in \mathbb{R}^{+}} \sigma_{\max }[G(i \omega)]
$$

Para um sistema tipo SISO, a norma $\mathcal{H}_{\infty}$ da função de transferência é igual ao máximo valor de $G(s)$. A figura 15 mostra um exemplo gráfico da norma $\mathcal{H}_{\infty}$ da função de transferência de um sistema SISO.

Figura 15 - Exemplo gráfico da norma $\|G\|_{\mathcal{H}_{\infty}}$ de um sistema SISO.

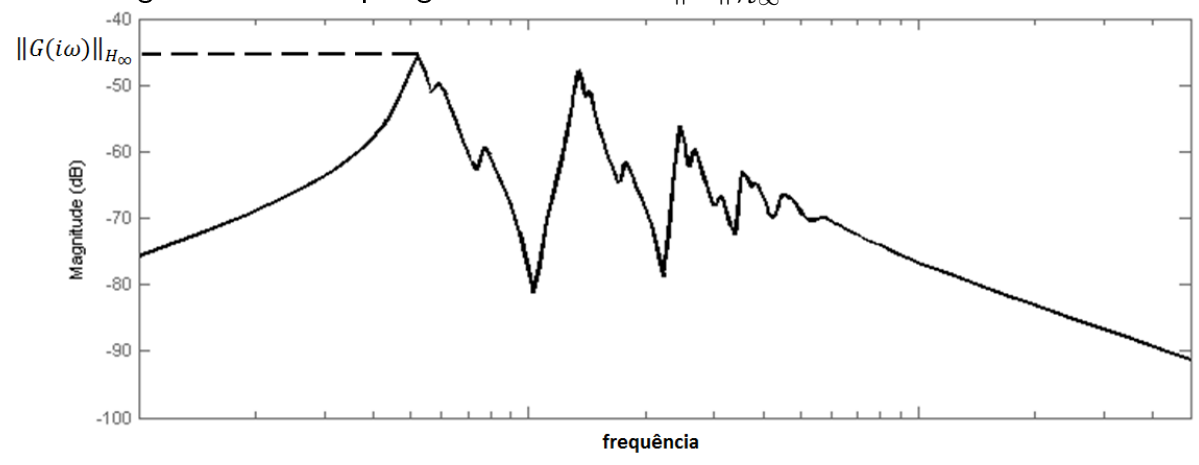

Cabe lembrar que este conceito somente se aplica a funções que possuem um limite superior finito da função de transferência, ou seja, sistemas assintoticamente estáveis (ANTOULAS, 2005).

Sabendo que a função de transferência $G(i \omega)$ de um sistema é aproximadamente igual à função de transferência $G_{k}(i \omega)$ do k-ésimo modo de vibrar para $\omega=\omega_{d k}$ (GAWRONSKI, 1998)

$$
G\left(i \omega_{d k}\right) \cong G_{k}\left(i \omega_{d k}\right)
$$

Gawronski mostra que a norma $\|G(s)\|_{\mathcal{H}_{\infty}}$ pode ser aproximada através da equação 182.

$$
\|G(s)\|_{\mathcal{H}_{\infty}} \cong \max _{k=1, \ldots, n_{m}}\left\|G_{k}(s)\right\|_{\mathcal{H}_{\infty}}=\max _{k=1, \ldots, n_{m}} \sigma_{\max }\left(G_{k}\left(\omega_{d k}\right)\right)
$$


Onde $G_{k}(s)$ é a função de transferência descrita pela equação 59 e $\omega_{d k}$ é a frequência natural amortecida, ambas referentes ao k-ésimo modo de vibrar.

A métrica $\mathcal{M}_{\mathcal{H}_{\infty}}$ proposta pelo autor, é igual à razão entre as normas $\mathcal{H}_{\infty}$ das funções de transferência do erro e do SC.

$$
\mathcal{M}_{\mathcal{H}_{\infty}}=\frac{\left\|G_{e}\right\|_{\mathcal{H}_{\infty}}}{\|G\|_{\mathcal{H}_{\infty}}}
$$

Portanto quando $\mathcal{M}_{\mathcal{H}_{\infty}}=0$, as normas $\mathcal{H}_{\infty}$ das funções de transferência do sistema reduzido e completo são idênticas $\left\|G_{r}\right\|_{\mathcal{H}_{\infty}}=\|G\|_{\mathcal{H}_{\infty}}$.

\subsubsection{Maior valor singular da função de transferência}

Ao se analisar as amplitudes do diagrama de Bode de um modelo reduzido tipo MIMO com $n_{u}$ entradas e $n_{y}$ saídas, a quantidade de gráficos a serem comparados é igual ao produto $n_{u} n_{y}$. Em consequência, avaliação das similaridades entre os sistemas é dificultada pela quantidade de informação a ser analisada.

Com objetivo de tornar a comparação mais objetiva, pode-se analisar os maiores valores singulares da função de transferência $G_{e}(s)$ do erro do SR. Desta maneira, mesmo para sistemas MIMO, apenas uma curva para cada SR comparado deverá ser analisada.

Neste trabalho, serão usados os maiores valores singulares das funções de transferência $G_{e}(s)$ do erro para comparação entre modelos reduzidos.

$$
G_{\sigma e}(s)=\sigma_{\max }\left(G_{e}(s)\right)
$$

Os valores de $G_{\sigma e}(s)$ representam o maior valor singular da função de transferência para cada valor de $s=i \omega$ sendo $\omega \in \mathbb{R}^{+}$.

Com a utilização de $G_{\sigma e}(s)$ para a comparação das respostas de sistemas dinâmicos no domínio da frequência, dois valores merecem destaque pois podem ser utilizados como métricas de comparação entre modelos reduzidos: $G_{\sigma e}(0)$ e $G_{\sigma e}(\infty)$, calculados de acordo com as equações 185 e 186 respectivamente.

$$
\begin{gathered}
\sigma_{\max }\left(G_{e}(\infty)\right)=\sigma_{\max }\left(D-D_{r}\right) \\
\sigma_{\max }\left(G_{e}(0)\right)=\sigma_{\max }\left(-C A^{-1} B-C_{r} A_{r}^{-1} B_{r}+D-D_{r}\right)
\end{gathered}
$$

Os valores de $\sigma_{\max }\left(G_{e}(0)\right)$ e $\sigma_{\max }\left(G_{e}(\infty)\right)$ quantificam a capacidade que um modelo reduzido tem de reproduzir a resposta da função de transferência do SC em frequências próximas de $\omega=0$ e $\omega \rightarrow \infty$ respectivamente. 
Portanto quando $\sigma_{\max }\left(G_{e}(0)\right)=0$, o valor das funções de transferência do sistema reduzido e completo são idênticas para $\omega=0$. De maneira análoga, $\sigma_{\max }\left(G_{e}(\infty)\right)$, o valor das funções de transferência do sistema reduzido e completo são idênticas para $\omega \rightarrow \infty$.

\subsection{RESPOSTA ESTÁTICA}

O erro da resposta estática pode ser calculado através da equação 24 . Entretanto, o autor sugere a utilização de uma métrica $\mathcal{M}_{e q}$ determinada pela razão entre as normas do erro da resposta estática $e_{e q}$ e da entrada estática $u_{e q}$. Desta maneira, a norma do erro estático é normalizada pela entrada.

$$
\mathcal{M}_{e q}=\frac{\left\|e_{e q}\right\|}{\left\|u_{e q}\right\|}
$$

Esta métrica quantifica a capacidade que um SR tem de aproximar a resposta da condição de equilíbrio do SC quando ambos são submetidos a uma mesma entrada constante $u(t)=u_{e q}$.

Uma outra maneira para avaliar a capacidade que o modelo reduzido tem de reproduzir a resposta estática do SC é através da métrica $\mathcal{M}_{\text {eqmax }}$. Esta métrica é a razão entre as normas $\left\|e_{e q}\right\|$ e $\left\|u_{e q}\right\|$ na pior situação possível, para uma entrada qualquer tal que $\left\|u_{e q}\right\|=1$.

A partir da equação 24 pode-se escrever o vetor de respostas como:

$$
y_{e q}=-C A^{-1} B+D
$$

A equação 188 pode ser utilizada de maneira análoga para o SR. Desta maneira, pode-se calcular o valor de $e_{e q}$ como:

$$
e_{e q}=\left(-C A^{-1} B+C_{r} A_{r}^{-1} B_{r}+D-D_{r}\right) u_{e q}
$$

A partir da equação 189 pode-se encontrar a situação onde o valor da norma $\left\|e_{e q}\right\|$ é máximo, considerando todas as entradas possíveis tal que $\left\|u_{e q}\right\|=1$.

$$
\mathcal{M}_{\text {eqmax }}=\max _{u_{\text {eq }} \neq 0}\left\|e_{e q}\right\|=\sigma_{\max }\left(G_{e}(0)\right)
$$

Portanto quando $\mathcal{M}_{\text {eqmax }}=0$, o valor das respostas estáticas do sistema reduzido e completo são idênticas para qualquer entrada estática $u_{e q}$.

\subsection{LIMITAÇÕES DAS TÉCNICAS APRESENTADAS}

Baseado nas métricas de representatividade descritas nos itens 9.1 a 9.3 pode-se avaliar a resposta de diferentes modelos reduzidos e selecionar o modelo mais adequado para uma 
determinada aplicação. Para isto, deve-se eleger quais serão as métricas que serão comparadas entre o SC e o SR, baseado nas características dinâmicas que devem ser priorizadas no SR.

Entretanto, será atribuído a cada SR um conjunto de valores escalares, um para cada métrica que foi eleita para servir de comparação.

A utilização de mais de uma métrica de comparação entre sistemas reduzidos pode levar a situações onde a determinação do SR mais adequado seja conflitante. A tabela 1 mostra um exemplo onde esta situação ocorre.

No exemplo da tabela 1] três modelos reduzidos diferentes foram obtidos a partir de um modelo de alta ordem. Determinou-se que o objetivo do SR nesta aplicação específica é reproduzir com a maior fidedignidade possível os sobre-sinais, a resposta transiente e a norma $\mathcal{H}_{\infty}$ do modelo completo. Para tal tarefa, as seguintes métricas foram eleitas para selecionar o modelo reduzido mais adequado: $\mathcal{M}_{s s}, \mathcal{M}_{\mathcal{L}_{2}}$ e $\mathcal{M}_{\mathcal{H}_{\infty}}$.

Tabela 1 - Exemplo da comparação entre modelos reduzidos

\begin{tabular}{|c|c|c|c|}
\hline $\begin{array}{c}\text { Nome do } \\
\text { modelo reduzido }\end{array}$ & $\begin{array}{c}\text { Métrica } \\
\mathcal{M}_{s s}\end{array}$ & $\begin{array}{c}\text { Métrica } \\
\mathcal{M}_{\mathcal{L}_{2}}\end{array}$ & $\begin{array}{c}\text { Métrica } \\
\mathcal{M}_{\mathcal{H}_{\infty}}\end{array}$ \\
\hline Modelo 01 & $\mathbf{0 . 1}$ & 0.50 & 0.11 \\
\hline Modelo 02 & 0.3 & $\mathbf{0 . 4 5}$ & $\mathbf{0 . 1 0}$ \\
\hline Modelo 03 & 1.1 & 0.81 & 0.21 \\
\hline
\end{tabular}

Avaliando os três modelos reduzidos do exemplo da tabela 1, observa-se que os valores das métricas do modelo 03 são maiores que dos outros modelos. Consequentemente pode-se dizer que o modelo 03 possui pior desempenho em relação aos outros dois em todas as métricas selecionadas.

O mesmo raciocínio do parágrafo anterior não pode ser utilizado para determinar qual é o modelo reduzido mais adequado entre os modelos 01 e 02 . O modelo 02 possui valores menores para as métricas $\mathcal{M}_{\mathcal{L}_{2}}$ e $\mathcal{M}_{\mathcal{H}_{\infty}}$ em comparação com o modelo 01 . Entretanto o modelo 01 possui valor menor para a métrica $\mathcal{M}_{s s}$.

Como consequência, o problema descrito acima é uma situação conflituosa onde não se pode determinar objetivamente qual dos modelos é o mais adequado apenas por comparação das métricas selecionadas. 
9.5 NOVA PROPOSTA PARA QUANTIFICAR A REPRESENTATIVIDADE DE UM MODELO REDUZIDO

Como mostrado no exemplo da tabela 1, a utilização das técnicas apresentadas nos itens 9.1 a 9.3 pode levar a situações conflituosas que inviabilizam a determinação objetiva do modelo reduzido mais adequado.

Como contribuição do autor neste trabalho, é proposta uma nova técnica para quantificar a representatividade de um modelo reduzido. Esta técnica, denominada Critério da Representatividade Rotal (CRT), possibilita quantificar a representatividade total de um SR e viabiliza a comparação objetiva do desempenho de diversos sistemas reduzidos diferentes.

A representatividade total de um modelo reduzido será utilizada para determinar o desempenho do mesmo. Portanto o autor propõe uma definição para o desempenho de um modelo reduzido:

\section{O desempenho de um modelo reduzido é sua capacidade de reproduzir um conjunto de características do modelo completo arbitrariamente definidas.}

Para quantificar o desempenho de um SR, é atribuído um valor escalar ao mesmo, chamado de representatividade total $\mathcal{R}$ do SR. A representatividade total é calculada através da equação 191.

$$
\mathcal{R}=\left(\mathcal{M}^{T} P \mathcal{M}\right)^{-\frac{1}{2}}
$$

Onde $\mathcal{M}$, chamado de vetor de métricas, é um vetor cujas componentes são métricas arbitrariamente definidas e que correspondem às características que devem ser reproduzidas mais fidedignamente pelo modelo reduzido.

A matriz $P$ é uma matriz de ponderação cuja diagonal é composta por parâmetros arbitrariamente determinados para ponderar as métricas do vetor $\mathcal{M}$.

Desta maneira, quanto maior o valor de $\mathcal{R}$, maior a representatividade do modelo reduzido ou seja, melhor a capacidade que o modelo reduzido tem de reproduzir as características elencadas no vetor $\mathcal{M}$.

A tabela 2 mostra os valores da representatividade total, calculados segundo a equação 191 . dos modelos do exemplo do item 9.4 .

$\mathrm{O}$ vetor de métricas utilizado é $\mathcal{M}=\left(\mathcal{M}_{s s} ; \mathcal{M}_{\mathcal{L}_{2}} ; \mathcal{M}_{\mathcal{H}_{\infty}}\right)^{T}$ e corresponde às seguintes características da resposta do modelo completo: sobre-sinais causados por uma entrada degrau, resposta transiente e norma $\mathcal{H}_{\infty}$.

A matriz de ponderação utilizada é $P=\operatorname{diag}(3 ; 2 ; 1)$. 
Tabela 2 - Exemplo da comparação entre modelos reduzidos pelo CRT

\begin{tabular}{|c|c|c|c|c|}
\hline $\begin{array}{c}\text { Nome do } \\
\text { modelo reduzido }\end{array}$ & $\begin{array}{c}\text { Métrica } \\
\mathcal{M}_{s s}\end{array}$ & $\begin{array}{c}\text { Métrica } \\
\mathcal{M}_{\mathcal{L}_{2}}\end{array}$ & $\begin{array}{c}\text { Métrica } \\
\mathcal{M}_{\mathcal{H}_{\infty}}\end{array}$ & $\begin{array}{c}\text { Representatividade } \\
\mathcal{R}\end{array}$ \\
\hline Modelo 01 & 0.1 & 0.50 & 0.11 & $\mathbf{1 . 3 5 8}$ \\
\hline Modelo 02 & 0.3 & 0.45 & 0.10 & 1.208 \\
\hline Modelo 03 & 1.1 & 0.81 & 0.21 & 0.448 \\
\hline
\end{tabular}

De acordo com a tabela 2 , o modelo 01 possui o maior desempenho entre os modelos avaliados. Portanto o modelo 01 é o mais adequado, dentre os três analisados, para estimar os sobre-sinais causados por uma entrada degrau, a resposta transiente e a norma $\mathcal{H}_{\infty}$ do modelo completo. 


\section{ESTUDOS DE CASO}

Neste capítulo será apresentada a descrição dos modelos usados para estudo e comparação de diferentes métodos de redução. O sistema completo, considerado neste trabalho como de alta ordem, será submetido à reduções segundo a metodologia de redução proposta no capítulo 4.

Foram construídos dois modelos para que sejam implementados os métodos de redução: um modelo para estudo da dinâmica vertical de veículos de passeio e um modelo para estudo da dinâmica longitudinal de um trem.

O modelo de veículo apresentado no item 10.1 possui uma quantidade consideravelmente pequena de GDL para que o leitor possa observar com mais detalhes todos os processos envolvidos na redução da ordem de um modelo. O modelo de trem apresentado no item 10.2 possui uma quantidade maior de GDL e é uma aplicação onde o uso de técnicas de redução é vantajoso.

\subsection{MODELO PARA ESTUDO DA DINÂMICA VERTICAL DE VEÍCULOS DE PASSEIO}

Foi elaborado um modelo de veículo com quatro rodas, para cálculo de dinâmica vertical como pode ser encontrado em (JAZAR, 2013). Para tal, foi deduzido um sistema de segunda ordem com $7 \mathrm{GDL}$ que posteriormente foi transformado em um sistema de primeira ordem com 14 GDL. Este modelo pode ser representado de acordo com a figura 16. Para construção do modelo, foram usados parâmetros que representam um carro de passeio com suspensões independentes e pneus.

As irregularidades da pista que o veículo percorre foram modeladas como entradas do sistema. Estas irregularidades são realizadas apenas na direção vertical e são representadas pelas variáveis $u_{1}, u_{2}, u_{3}$ e $u_{4}$.

As equações diferenciais de segunda ordem que descrevem o sistema foram deduzidas utilizando técnica de sistemas multicorpos descrita no apêndice 15. Os graus de liberdade do sistema de segunda ordem são:

- Z : Deslocamento vertical do chassi

- $\phi$ : ângulo de rolagem do chassi

- $\theta$ : ângulo de arfagem do chassi

- $Z_{1}$ : Deslocamento vertical do centro da roda dianteira esquerda

- $Z_{2}$ : Deslocamento vertical do centro da roda dianteira direita 
Figura 16 - Modelo de veículo de passeio

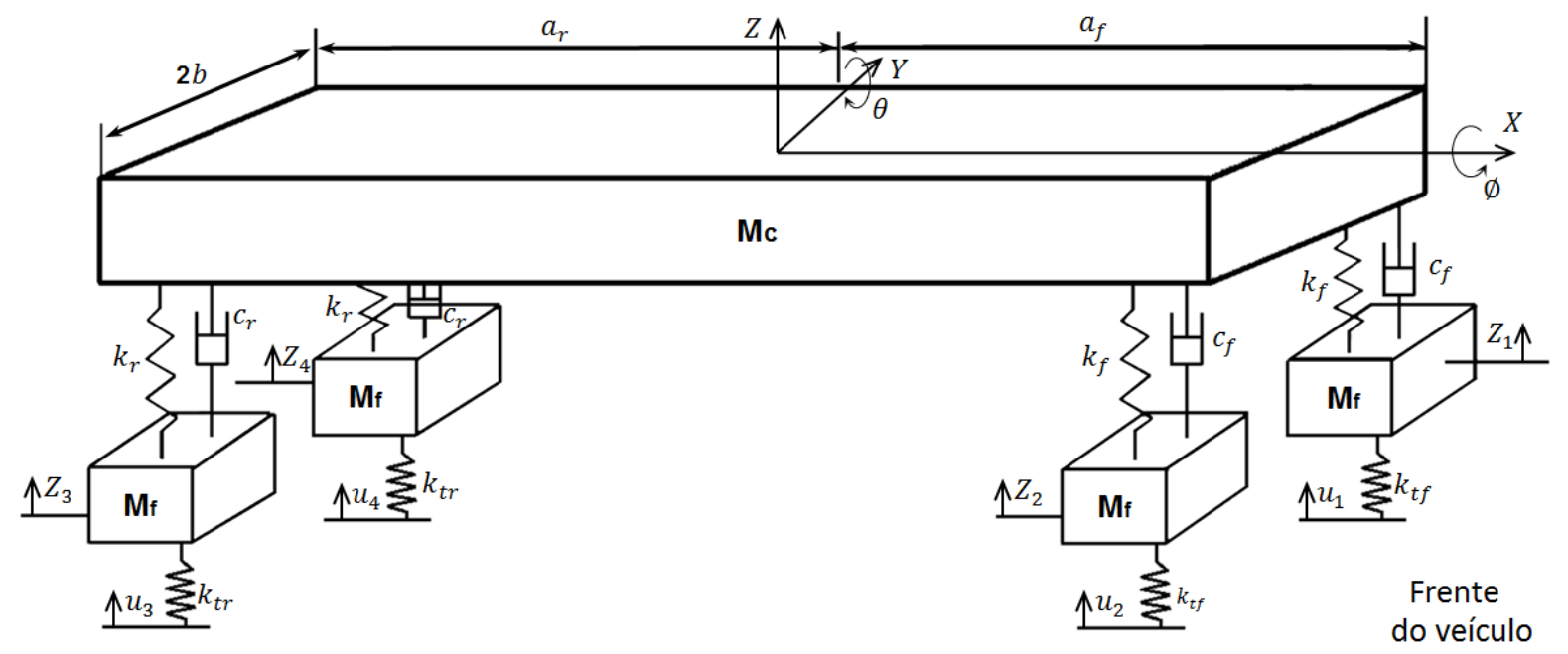

- $Z_{3}$ : Deslocamento vertical do centro da roda traseira direita

- $Z_{4}$ : Deslocamento vertical do centro da roda traseira esquerda

O vetor $q$ define os GDL no sistema de segunda ordem pode ser escrito na forma:

$$
q=\left[\begin{array}{lllllll}
Z & \phi & \theta & Z_{1} & Z_{2} & Z_{3} & Z_{4}
\end{array}\right]^{T}
$$

As equações que descrevem as energias cinética e potencial do sistema são mostradas respectivamente em 193 e 194.

$$
\begin{array}{r}
T=\frac{1}{2} m_{c} \dot{Z}^{2}+\frac{1}{2} I_{x} \dot{\phi}^{2}+\frac{1}{2} I_{y} \dot{\theta}^{2}+\frac{1}{2} m_{f}\left(\dot{Z}_{1}^{2}+\dot{Z}_{2}^{2}\right)+\frac{1}{2} m_{r}\left(\dot{Z}_{3}^{2}+\dot{Z}_{4}^{2}\right) \\
V=\frac{1}{2} k_{f}\left(Z-Z_{1}+b \phi-a_{f} \theta\right)^{2}+\frac{1}{2} k_{f}\left(Z-Z_{2}-b \phi-a_{f} \theta\right)^{2}+ \\
+\frac{1}{2} k_{r}\left(Z-Z_{3}-b \phi+a_{r} \theta\right)^{2}+\frac{1}{2} k_{r}\left(Z-Z_{4}+b \phi+a_{f} \theta\right)^{2}+\frac{1}{2} k_{t f}\left(Z_{1}-Z_{r 1}\right)^{2}+ \\
+\frac{1}{2} k_{t f}\left(Z_{2}-Z_{r 2}\right)^{2}+\frac{1}{2} k_{t r}\left(Z_{3}-Z_{r 3}\right)^{2}+\frac{1}{2} k_{t r}\left(Z_{4}-Z_{r 4}\right)^{2}
\end{array}
$$

A função de Rayleigh para o modelo em questão foi calculada de acordo com a equação 255

$$
\begin{aligned}
R=\frac{1}{2} c_{f}\left(\dot{Z}-\dot{Z}_{1}+b \dot{\phi}-\right. & \left.a_{f} \dot{\theta}\right)^{2}+\frac{1}{2} c_{f}\left(\dot{Z}-\dot{Z}_{2}-b \dot{\phi}-a_{f} \dot{\theta}\right)^{2}+ \\
& +\frac{1}{2} c_{r}\left(\dot{Z}-\dot{Z}_{3}-b \dot{\phi}+a_{f} \dot{\theta}\right)^{2}+\frac{1}{2} c_{r}\left(\dot{Z}-\dot{Z}_{4}+b \dot{\phi}+a_{f} \dot{\theta}\right)^{2}
\end{aligned}
$$


A equação de Lagrange 251. foi usada para calcular as matrizes de massa, amortecimento e rigidez do sistema ( $M, C_{a m}$ e $K$ respectivamente). Desta maneira, pode-se escrever o modelo na forma

$$
\begin{aligned}
& M \ddot{q}+C_{a m} \dot{q}+K q=F u \\
& M=\left[\begin{array}{ccccccc}
m_{c} & 0 & 0 & 0 & 0 & 0 & 0 \\
0 & I_{x} & 0 & 0 & 0 & 0 & 0 \\
0 & 0 & I_{y} & 0 & 0 & 0 & 0 \\
0 & 0 & 0 & m_{f} & 0 & 0 & 0 \\
0 & 0 & 0 & 0 & m_{f} & 0 & 0 \\
0 & 0 & 0 & 0 & 0 & m_{t} & 0 \\
0 & 0 & 0 & 0 & 0 & 0 & m_{t}
\end{array}\right] \\
& C_{a m}=\left[\begin{array}{ccc}
2\left(c_{f}+c_{r}\right) & 0 & 2\left(-a_{f} c_{f}+a_{r} c_{r}\right) \\
0 & 2 b^{2}\left(c_{f}+c_{r}\right) & 0 \\
2\left(-a_{f} c_{f}+a_{r} c_{r}\right) & 0 & 2\left(a_{f}^{2} c_{f}+a_{r}^{2} c_{r}\right) \\
-c_{f} & -b c_{f} & a_{f} c_{f} \\
-c_{f} & b c_{f} & a_{f} c_{f} \\
-c_{r} & b c_{r} & -a_{r} c_{r} \\
-c_{r} & -b c_{r} & -a_{r} c_{r}
\end{array}\right. \\
& \left.\begin{array}{cccc}
-c_{f} & -c_{f} & -c_{r} & -c_{r} \\
-b c_{f} & b c_{f} & b c_{r} & -b c_{r} \\
a_{f} c_{f} & a_{f} c_{f} & -a_{r} c_{r} & -a_{r} c_{r} \\
c_{f} & 0 & 0 & 0 \\
0 & c_{f} & 0 & 0 \\
0 & 0 & c_{r} & 0 \\
0 & 0 & 0 & c_{r}
\end{array}\right]
\end{aligned}
$$




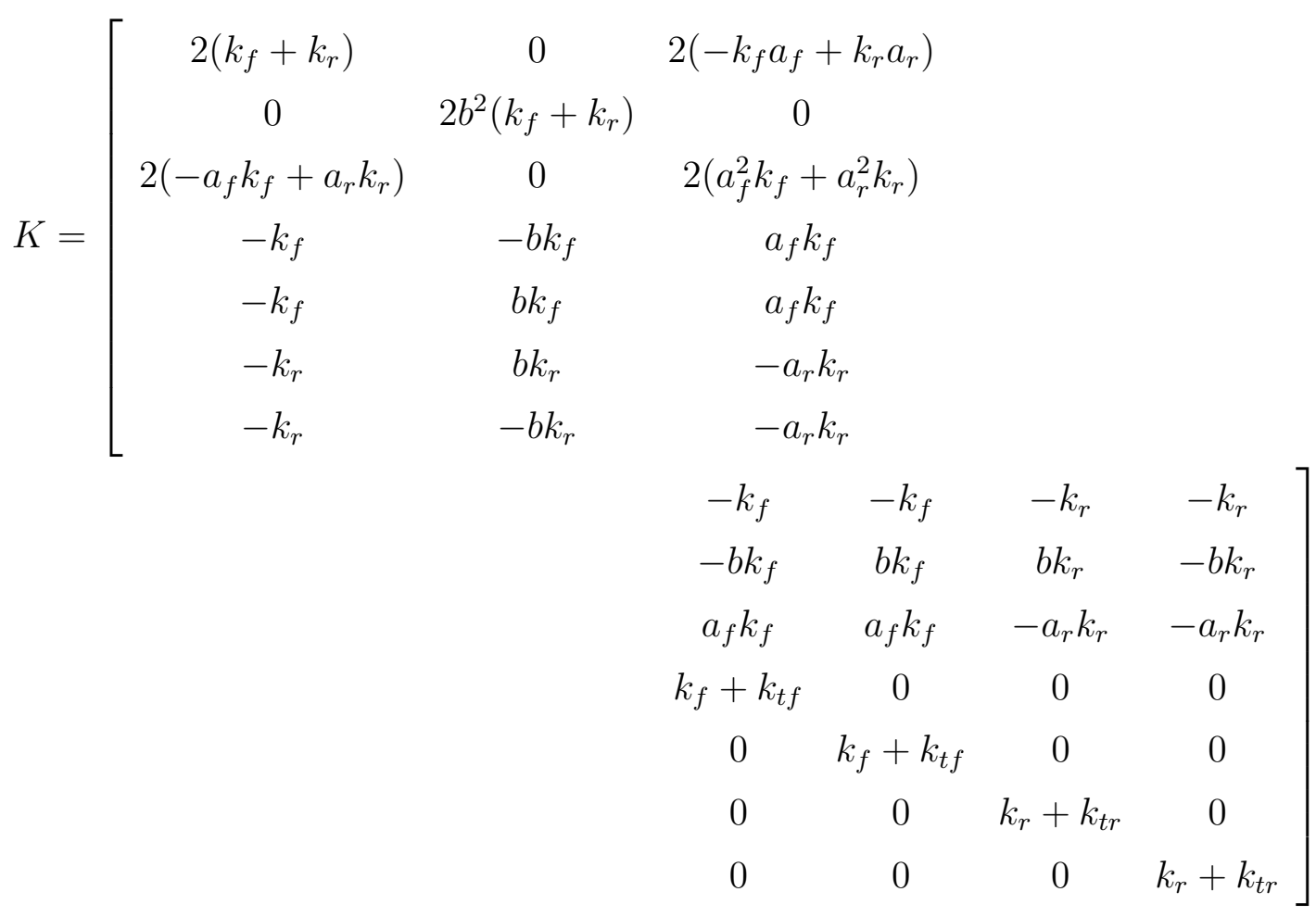

$$
\begin{aligned}
& F=\left[\begin{array}{cccc}
0 & 0 & 0 & 0 \\
0 & 0 & 0 & 0 \\
0 & 0 & 0 & 0 \\
k_{t f} & 0 & 0 & 0 \\
0 & k_{t f} & 0 & 0 \\
0 & 0 & k_{t r} & 0 \\
0 & 0 & 0 & k_{t r}
\end{array}\right]
\end{aligned}
$$

Os valores dos parâmetros usados para a simulação do modelo de veículo completo estão especificados na tabela 3 .

O sistema dinâmico pode ser escrito através de equações de primeira ordem, na forma da equação 201 .

$$
\left\{\begin{array}{l}
\dot{x}=A x+B u \\
y=C x+D u
\end{array}\right.
$$

Esta forma é chamada de espaço de estados. O vetor de estados do sistema em espaço de estados é definido da seguinte maneira:

$$
x=\left\{\begin{array}{l}
q \\
\dot{q}
\end{array}\right\}
$$

As matrizes $A, B, C$ e $D$ do sistema de primeira ordem são calculadas da seguinte maneira: 
Tabela 3 - Dados de entrada do modelo de veículo de passeio

\begin{tabular}{|l|c|c|c|}
\hline Parâmetro & Variável & Unidade & Valor \\
\hline Massa do Chassi & $m_{c}$ & $k g$ & 1136 \\
\hline Momento de inércia de rolagem & $I_{x}$ & $k g m^{2}$ & 600 \\
\hline Momento de inércia de arfagem & $I_{y}$ & $k g m^{2}$ & 2400 \\
\hline Massa de cada roda dianteira & $m_{f}$ & $k g$ & 60 \\
\hline Massa de cada roda traseira & $m_{r}$ & $k g$ & 60 \\
\hline Rigidez da mola dianteira & $k_{f}$ & $\mathrm{~N} / \mathrm{m}$ & 20620 \\
\hline Rigidez da mola traseira & $k_{r}$ & $\mathrm{~N} / \mathrm{m}$ & 20620 \\
\hline Rigidez do amortecedor dianteiro & $c_{f}$ & $\mathrm{~N} /(\mathrm{m} / \mathrm{s})$ & 3924 \\
\hline Rigidez do amortecedor traseiro & $c_{r}$ & $\mathrm{~N} /(\mathrm{m} / \mathrm{s})$ & 2943 \\
\hline Rigidez do pneu dianteiro & $k_{t f}$ & $\mathrm{~N} / \mathrm{m}$ & 190000 \\
\hline Rigidez do pneu traseiro & $k_{t r}$ & $\mathrm{~N} / \mathrm{m}$ & 190000 \\
\hline $\begin{array}{l}\text { Distância longitudinal entre centro de massa do } \\
\text { chassi e eixo dianteiro }\end{array}$ & $a_{f}$ & $\mathrm{~m}$ & 1.15 \\
\hline $\begin{array}{l}\text { Distância longitudinal entre centro de massa do } \\
\text { chassi e eixo traseiro }\end{array}$ & $a_{r}$ & $\mathrm{~m}$ & 1.65 \\
\hline $\begin{array}{l}\text { Distância lateral entre centro de massa do } \\
\text { chassi e centros das rodas }\end{array}$ & $b$ & $\mathrm{~m}$ & 0.9 \\
\hline
\end{tabular}

$$
\begin{aligned}
A & =\left[\begin{array}{ccc}
0 & I \\
-M^{-1} K & -M^{-1} C_{a m}
\end{array}\right] \\
B & =\left[\begin{array}{c}
0 \\
M^{-1} F
\end{array}\right] \\
C & =\left[\begin{array}{llllllllllllll}
1 & 0 & 0 & 0 & 0 & 0 & 0 & 0 & 0 & 0 & 0 & 0 & 0 & 0 \\
0 & 1 & 0 & 0 & 0 & 0 & 0 & 0 & 0 & 0 & 0 & 0 & 0 & 0 \\
0 & 0 & 1 & 0 & 0 & 0 & 0 & 0 & 0 & 0 & 0 & 0 & 0 & 0
\end{array}\right] \\
D & =\left[\begin{array}{llll}
0 & 0 & 0 & 0 \\
0 & 0 & 0 & 0 \\
0 & 0 & 0 & 0
\end{array}\right]
\end{aligned}
$$

A matriz $C$ foi construída de maneira que os componentes da saída $y(t)=\left[y_{1}(t) y_{2}(t) y_{3}(t)\right]^{T}=$ $[Z(t) \phi(t) \theta(t)]^{T}$ sejam respectivamente as respostas temporais dos movimentos de deslocamento vertical $Z(t)$, ângulo de rolagem $\phi(t)$ e ângulo de arfagem $\theta(t)$ do chassi do veículo. 


\subsection{MODELO PARA ESTUDO DA DINÂMICA LONGITUDINAL DE UM TREM}

Baseado no modelo para estudo da dinâmica longitudinal de um trem, descrito por (BARBOSA, 1993), que descreve um trem composto por uma série de veículos interligados entre si formando uma longa corrente.

Os veículos são considerados corpos rígidos e estão conectados através de um acoplamento. Cada extremidade do acoplamento se liga a um veículo através de uma mola e um amortecedor, como mostrado na figura 17

Figura 17 - Acoplamento entre veículos ferroviários. Fonte: (BARBOSA, 1993)

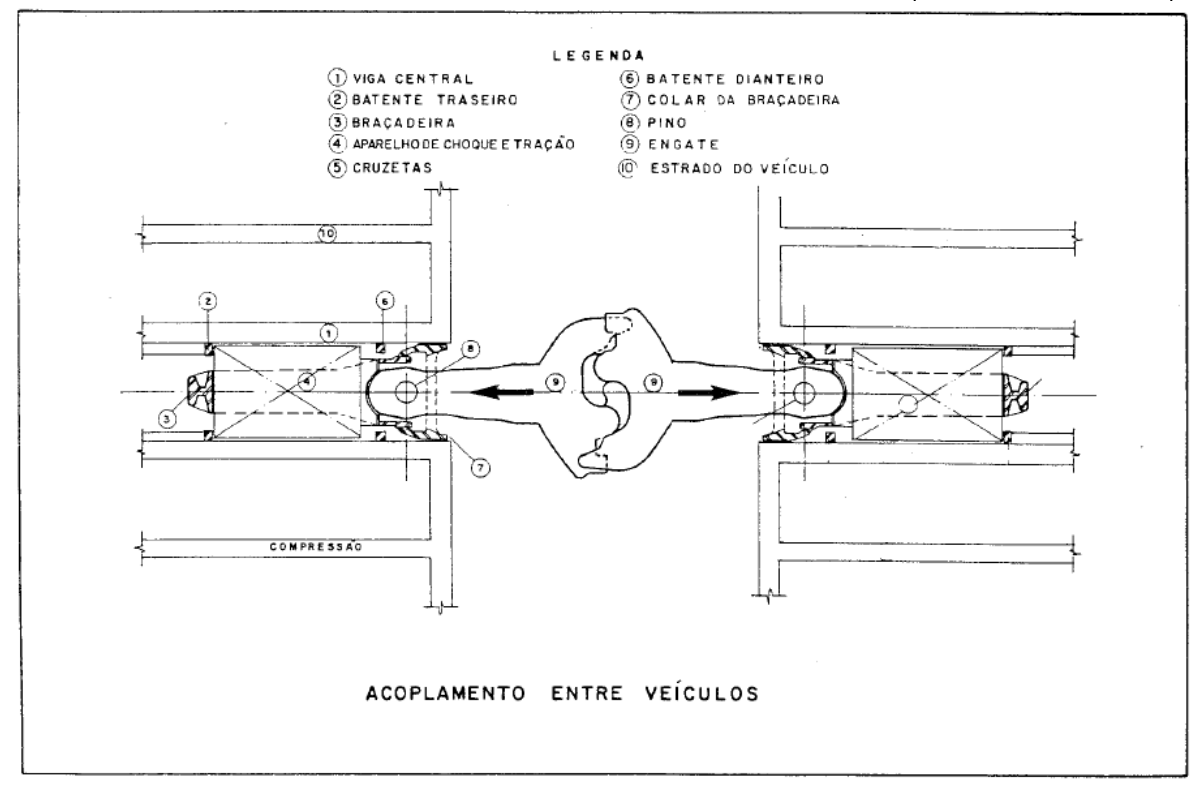

Este modelo tem como objetivo a investigação dos efeitos exclusivamente na direção longitudinal do trem, portanto será considerado que cada veículo é composto de uma massa concentrada com deslocamentos na direção longitudinal, como mostrado na figura 18 .

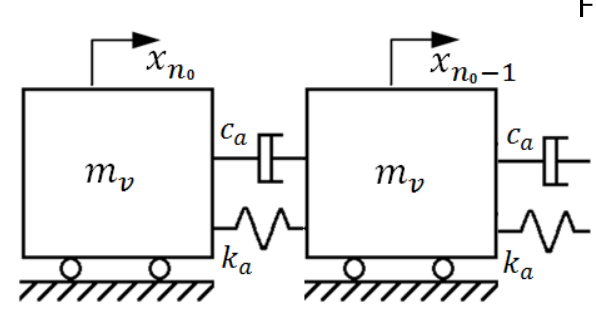

Figura 18 - Modelo de trem.

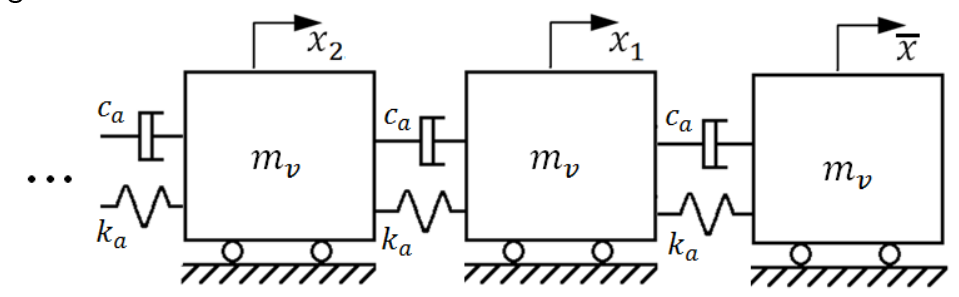

Será considerado um trem com um total de 200 veículos, sendo que os 4 primeiros são locomotivas, seguidos de 194 vagões e mais 2 locomotivas. Tanto os vagões quanto as locomotivas têm massa $m_{v}$. As locomotivas possuem sistema de tração que movimenta o trem e que exerce sobre cada locomotiva uma força longitudinal resultante $F_{t}$, mostrada na 
figura 19

Figura 19 - Forças externas do modelo, representando tração das locomotivas.

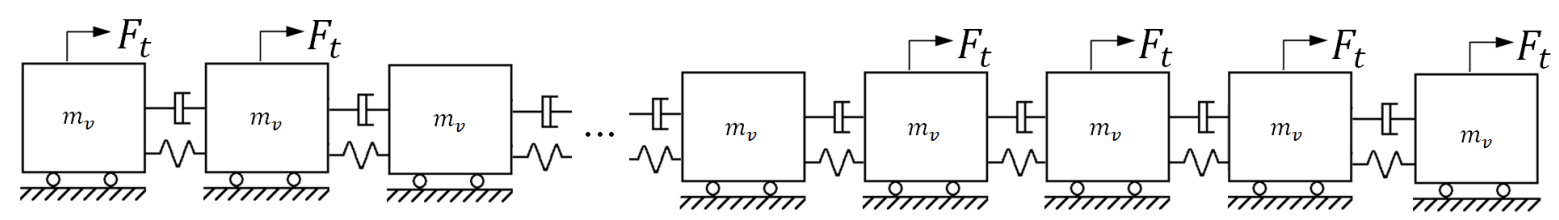

O vetor de estados do sistema foi construído de forma que cada estado representa a distância de um corpo rígido até o primeiro veículo: $Z_{i}=x_{i}-\bar{x}$. Onde $\bar{x}$ é o deslocamento longitudinal do primeiro veículo.

$$
Z=\left[\begin{array}{lllllll}
Z_{1} & \dot{Z} & Z_{2} & \dot{Z}_{2} & \ldots & Z_{n_{0}} & \dot{Z}_{n_{0}}
\end{array}\right]^{T}
$$

Onde $n_{0}=199$ e o número de GDL no sistema de primeira ordem em espaço de estados é $n=398$.

A dedução das equações de movimento do trem foi realizada utilizando a técnica de multicorpos, de maneira equivalente ao do veículo no item 10.1. Como a quantidade de GDL neste modelo é elevada, o processo de dedução das equações de movimento não será mostrado com detalhes.

Foi considerado que o sistema de tração é acionado simultaneamente em todas as locomotivas, desta maneira o sistema possui apenas uma entrada $u(t)=F_{t}(t)$, portanto a matriz $B$ do sistema em espaço de estados tem apenas uma coluna.

A matriz $C$ do sistema foi construída de maneira que o vetor de respostas tenha dimensão $n_{y}=8$ e cada componente $y_{i}(t)$ mostra a força exercida no veículo pelo veículo a sua frente. As componentes de resposta $y(t)=\left[y_{1}(t), \ldots, y_{8}(t)\right]^{T}$ mostram as forças nos veículos 3,20 , 50, 100, 150, 180, 197 e 200 .

$$
y(t)=\left\{\begin{array}{l}
k\left(Z_{1}-Z_{2}\right)+c\left(\dot{Z}_{1}-\dot{Z}_{2}\right) \\
k\left(Z_{18}-Z_{19}\right)+c\left(\dot{Z}_{18}-\dot{Z}_{19}\right) \\
k\left(Z_{48}-Z_{49}\right)+c\left(\dot{Z}_{48}-\dot{Z}_{49}\right) \\
k\left(Z_{98}-Z_{99}\right)+c\left(\dot{Z}_{98}-\dot{Z}_{99}\right) \\
k\left(Z_{148}-Z_{149}\right)+c\left(\dot{Z}_{148}-\dot{Z}_{149}\right) \\
k\left(Z_{178}-Z_{179}\right)+c\left(\dot{Z}_{178}-\dot{Z}_{179}\right) \\
k\left(Z_{195}-Z_{196}\right)+c\left(\dot{Z}_{195}-\dot{Z}_{196}\right) \\
k\left(Z_{198}-Z_{199}\right)+c\left(\dot{Z}_{198}-\dot{Z}_{199}\right)
\end{array}\right\}
$$

A matriz $D$ do sistema em espaço de estados tem dimensão $8 \times 1$ e tem todos os coeficientes nulos. 
Os valores dos parâmetros usados para a simulação do modelo de trem estão especificados na tabela 4

Tabela 4 - Dados de entrada do modelo de trem

\begin{tabular}{|l|c|c|c|}
\hline Parâmetro & Variável & Unidade & Valor \\
\hline Massa de cada veículo & $m_{v}$ & $k g$ & 120000 \\
\hline Rigidez da mola dos acoplamentos & $k_{a}$ & $\mathrm{~N} / \mathrm{m}$ & $105 \cdot 10^{5}$ \\
\hline Rigidez do amortecedor dos acoplamentos & $c_{a}$ & $\mathrm{~N} /(\mathrm{m} / \mathrm{s})$ & $80 \cdot 10^{3}$ \\
\hline
\end{tabular}




\section{TÉCNICAS DE REDUÇÃO IMPLEMENTADAS}

Com o objetivo de comparar os procedimentos apresentados neste trabalho, o autor construiu modelos reduzidos distintos, todos baseados na metodologia para redução da ordem de modelos proposta no capítulo 4

Como mostrado no capítulo 4 os 4 principais processos envolvidos na redução são:

- Mudança de base

- Escolha e implementação do critério para seleção dos GDL que serão eliminados

- Escolha e implementação de um método de eliminação

- Correções do modelo reduzido

Como o número de combinações possíveis entre os procedimentos mostrados é grande, foram elaborados 8 modelos reduzidos para que diferentes técnicas de redução sejam analisadas e comparadas. Cada modelo reduzido foi construído como mostrado na tabela 5 que especifica as escolhas realizadas em cada um dos 4 processos citados acima.

Tabela 5 - Métodos implementados

\begin{tabular}{|c|c|c|c|c|}
\hline $\begin{array}{c}\text { Técnica } \\
\text { implementada }\end{array}$ & $\begin{array}{c}\text { Base } \\
\text { de projeção }\end{array}$ & $\begin{array}{c}\text { Critério } \\
\text { de seleção }\end{array}$ & $\begin{array}{c}\text { Método } \\
\text { de eliminação }\end{array}$ & $\begin{array}{c}\text { Método } \\
\text { de correção }\end{array}$ \\
\hline Técnica R1 & Base original & $\begin{array}{c}\text { conhecimento } \\
\text { prévio }\end{array}$ & ETD & - \\
\hline Técnica R2 & BFM & CSCO & ETD & - \\
\hline Técnica R3 & BFM & CSCO & EPS & - \\
\hline Técnica R4 & Base DVS & CSVS & ETD & - \\
\hline Técnica R5 & BKMA & CSVK & ETD & - \\
\hline Técnica R6 & $\begin{array}{c}\text { Realização } \\
\text { balanceada }\end{array}$ & CSNH & ETD & - \\
\hline Técnica R7 & $\begin{array}{c}\text { Realização } \\
\text { balanceada }\end{array}$ & CSNH & EPS & - \\
\hline Técnica R8 & BFM & CSCO & ETD & Correção de \\
& & & & $G_{r}\left(s_{0}\right)$ por $D_{r}$ \\
\hline
\end{tabular}

Os modelos reduzidos calculados a partir de cada uma das técnicas acima serão chamados neste trabalho pelos nomes "modelo MRi", onde $i=1, \ldots, 8$

Nos itens 11.1 a 11.8 serão apresentadas características específicas de cada uma das técnicas de redução elencadas na tabela 5 . 


\subsection{TÉCNICA R1}

A denominada técnica R1 foi implementada na base original em que o sistema foi descrito no capítulo 10 A seleção de variáveis candidatas a serem eliminadas foi realizada baseada no conhecimento prévio do autor, pois neste caso não é possível aplicar nenhum dos outros critérios de seleção descritos no capítulo 6 .

O método de eliminação utilizado foi o de truncamento direto e não foi implementado nenhum método de correção da resposta do sistema reduzido.

Está é a técnica que requer o menor custo computacional para sua implementação dentre todos os 7 implementados neste trabalho. Isso pode ser percebido pois não é realizada nenhuma mudança de base do sistema, não são usados recursos computacionais para a seleção de veriáveis a serem eliminadas e a eliminação acontece pelo simples truncamento das variáveis eliminadas. Além disso, o SR não é corrigido após a eliminação das variáveis, o que também não exige recurso computacional excessivo.

Baseado nas discussões apresentadas previamente neste trabalho, pode-se esperar as seguintes características para esta técnica:

- Como foi utilizado o método de ETD, o SR fornece uma boa aproximação da função de transferência do SC quando avaliado em altas frequências $G(\infty)=G_{r}(\infty)$.

\subsection{TÉCNICA R2}

Na denominada técnica R2 foi realizada inicialmente uma mudança de base para a BFM utilizando a equação 43. A seleção de GDL candidatos a serem eliminados foi realizada pelo critério CSCO, descrito no item 6.1.3. O método de eliminação utilizado foi o de truncamento direto (ETD) e não foi implementado nenhum método de correção da resposta do sistema reduzido.

O método de redução por truncamento de GDL na BFM também é conhecido como truncamento modal.

O sistema completo na BFM pode ser escrito como:

$$
\left\{\begin{array}{c}
\dot{x}_{1} \\
\dot{x}_{2} \\
\hline y
\end{array}\right\}=\left[\begin{array}{cc|c}
A_{11} & 0 & B_{1} \\
0 & A_{22} & B_{2} \\
\hline C_{1} & C_{2} & D
\end{array}\right]\left\{\begin{array}{c}
x_{1} \\
x_{2} \\
\hline u
\end{array}\right\}
$$


Portanto no caso da técnica R2, a equação 4 fica na forma

$$
\left\{\begin{array}{c}
\dot{x}_{1} \\
\dot{x}_{2} \\
\dot{x}_{r} \\
\hline e
\end{array}\right\}=\left[\begin{array}{ccc|c}
A_{11} & 0 & 0 & B_{1} \\
0 & A_{22} & 0 & B_{2} \\
0 & 0 & A_{11} & B_{1} \\
\hline C_{1} & C_{2} & -C_{1} & 0
\end{array}\right]\left\{\begin{array}{c}
x_{1} \\
x_{2} \\
x_{r} \\
\hline u
\end{array}\right\}
$$

Expandindo o sistema de equações acima, encontramos três sistemas cuja dinâmica é desacoplada:

$$
\begin{aligned}
& \left\{\begin{array}{c}
\dot{x}_{1} \\
\hline y_{1}
\end{array}\right\}=\left[\begin{array}{c|c}
A_{11} & B_{1} \\
\hline C_{1} & 0
\end{array}\right]\left\{\begin{array}{c}
x_{1} \\
\hline u
\end{array}\right\} \\
& \left\{\begin{array}{c}
\dot{x}_{2} \\
\hline y_{2}
\end{array}\right\}=\left[\begin{array}{c|c}
A_{22} & B_{2} \\
\hline C_{2} & 0
\end{array}\right]\left\{\begin{array}{c}
x_{2} \\
\hline u
\end{array}\right\} \\
& \left\{\begin{array}{c}
\dot{x}_{r} \\
\hline-y_{r}
\end{array}\right\}=\left[\begin{array}{c|c}
A_{11} & B_{1} \\
\hline-C_{1} & 0
\end{array}\right]\left\{\begin{array}{c}
x_{r} \\
\hline u
\end{array}\right\}
\end{aligned}
$$

Por comparação entre as equações 206 e 208 pode-se observar que nesta técnica, o vetor de estados do SR é idêntico aos estados mantidos do SC, ou seja: $x_{r}(t)=x_{1}(t)$.

Também por comparação, observa-se que os vetores de resposta $y_{1}(t)$ e $y_{r}(t)$ também são iguais, portanto o erro $e(t)$ é igual a

$$
e(t)=y(t)-y_{r}(t)=y_{1}(t)+y_{2}(t)-y_{r}(t)=y_{2}(t)
$$

Calculando função de transferência do erro $G_{e}(s)$, pode-se perceber que ela é igual à soma das funções de transferência dos modos truncados:

$$
G_{e}=G-G_{r}=\sum_{k=1}^{n_{m}} G_{i}-\sum_{k=1}^{n_{m r}} G_{i}+D-D_{r}=\sum_{k=n_{m r}+1}^{n_{m}} G_{i}=C_{2}\left(s I-A_{22}\right)^{-1} B_{2}
$$

onde $n_{m}$ é o número de modos de vibrar do $\mathrm{SC}$ e $n_{m r}$ é o número de modos do SR.

Portanto pode-se simplificar e equação 205 de maneira que o erro do SR pode ser calculado como:

$$
\left\{\begin{array}{c}
\dot{x}_{2} \\
\hline e
\end{array}\right\}=\left[\begin{array}{c|c}
A_{22} & B_{2} \\
\hline C_{2} & 0
\end{array}\right]\left\{\begin{array}{c}
x_{2} \\
\hline u
\end{array}\right\}
$$

Como a resposta $e(t)$ encontrada pelas equações 205 e 210 é idêntica, a utilização da segunda é mais indicada por exigir menor custo computacional. Isso ocorre pois a ordem do sistema da equação 205 é maior que da equação 210. 
O erro da resposta estática do modelo reduzido também pode ser calculado a partir da equação 210, assumindo $\dot{x}_{2}=0$

$$
\left[\begin{array}{c|c}
A_{22} & 0 \\
\hline C_{2} & -I
\end{array}\right]\left\{\frac{x_{2 e q}}{e_{e q}}\right\}=\left\{\frac{-B_{2}}{0}\right\} u_{e q}
$$

Pode-se perceber que o erro $e_{e q}$ é idêntico aos calculados pelas equações 24 e 211, sendo que a utilização da segunda é mais indicada por exigir menor custo computacional.

A partir da equação 182. Gawronski (GAWRONSKI, 1998) mostra uma maneira de calcular uma aproximação da norma $\left\|G_{e}\right\|_{\mathcal{H}_{\infty}}$, mostrada na equação 212 .

$$
\left\|G_{e}(s)\right\|_{\mathcal{H}_{\infty}} \cong \max _{\left(k=n_{m r}+1, \ldots, n_{m}\right)}\left\|G_{k}(s)\right\|_{\mathcal{H}_{\infty}}=\max _{\left(k=n_{m r}+1, \ldots, n_{m}\right)} \sigma_{m a x}\left(G_{k}\left(\omega_{d k}\right)\right)
$$

Também no mesmo trabalho, Gawronski mostra uma maneira de se calcular uma aproximação para a norma $\left\|G_{e}\right\|_{\mathcal{H}_{2}}$

$$
\left\|G_{e}(s)\right\|_{\mathcal{H}_{2}} \cong\left(\sum_{n_{m r}}^{n_{m}}\left\|G_{k}(s)\right\|_{\mathcal{H}_{2}}^{2}\right)^{\frac{1}{2}}
$$

Uma vantagem de utilizar as equações 212 e 213 é a possibilidade de se calcular uma aproximação para as normas $\left\|G_{e}\right\|_{\mathcal{H}_{\infty}}$ e $\left\|G_{e}\right\|_{\mathcal{H}_{2}}$ após a etapa de seleção dos GDL a serem eliminados e antes da eliminação dos GDL.

Analogamente, utilizando a equação 210 , pode-se provar que as equações 212 e 213 são válidas para todos os modelos reduzidos por truncagem modal desde que não seja aplicado nenhum método de correção.

Baseado nas discussões apresentadas previamente neste trabalho, pode-se esperar as seguintes características para esta técnica:

- As formas modais e polos dos modos preservados no SR serão iguais aos do SC.

- Caso o SC seja estável, o modelo reduzido também será estável.

- O vetor de estados do SR é idêntico aos estados mantidos do SC $x_{r}=x_{1}$.

- Como foi utilizado o método de ETD, o SR fornece uma boa aproximação da função de transferência do SC quando avaliado em altas frequências $G(\infty)=G_{r}(\infty)$.

- Existem 3 métricas que podem ser estimadas antes da eliminação dos GDL, descritas nas equações 211. 212 e 213. Isso pode auxiliar na determinação do número de GDL a serem reduzidos, o que permite que sejam utilizados os processos na parte esquerda do fluxograma da figura 1 . 


\subsection{TÉCNICA R3}

Na denominada técnica R3 foi realizada inicialmente uma mudança de base para a BFM utilizando a equação 43. A seleção de GDL candidatos a serem eliminados foi realizada pelo critério CSCO, descrito no item 6.1.3. O método de eliminação utilizado foi o EPS, descrito no item 7.3.1 e não foi implementado nenhum método de correção da resposta do SR.

O sistema completo na BFM pode ser escrito como na equação 204. Portanto utilizando a equação 144 pode-se calcular as matrizes do SR:

$$
\left\{\begin{array}{l}
A_{r}=A_{11}-A_{12} A_{22}^{-1} A_{21}=A_{11} \\
B_{r}=B_{1}-A_{12} A_{22}^{-1} B_{2}=B_{1} \\
C_{r}=C_{1}-C_{2} A_{22}^{-1} A_{21}=C_{1} \\
D_{r}=D-C_{2} A_{22}^{-1} B_{2}=-C_{2} A_{22}^{-1} B_{2}
\end{array}\right.
$$

Pois no sistema completo projetado na BFM, as submatrizes $A_{12}$ e $A_{21}$ são nulas $\left(A_{12}=\right.$ $\left.A_{21}=0\right)$.

No caso da técnica R3, a equação 4 fica na forma

$$
\left\{\begin{array}{c}
\dot{x}_{1} \\
\dot{x}_{2} \\
\dot{x}_{r} \\
\hline e
\end{array}\right\}=\left[\begin{array}{ccc|c}
A_{11} & 0 & 0 & B_{1} \\
0 & A_{22} & 0 & B_{2} \\
0 & 0 & A_{11} & B_{1} \\
\hline C_{1} & C_{2} & -C_{1} & C_{2} A_{22}^{-1} B_{2}
\end{array}\right]\left\{\begin{array}{c}
x_{1} \\
x_{2} \\
x_{r} \\
u
\end{array}\right\}
$$

Expandindo a equação acima, pode-se escrever três sistemas desacoplados:

$$
\begin{aligned}
& \left\{\begin{array}{c}
\dot{x}_{1} \\
\hline y_{1}
\end{array}\right\}=\left[\begin{array}{c|c}
A_{11} & B_{1} \\
\hline C_{1} & 0
\end{array}\right]\left\{\begin{array}{c}
x_{1} \\
\hline u
\end{array}\right. \\
& \left\{\begin{array}{c}
\dot{x}_{2} \\
\hline y_{2}
\end{array}\right\}=\left[\begin{array}{c|c}
A_{22} & B_{2} \\
\hline C_{2} & 0
\end{array}\right]\left\{\begin{array}{c}
x_{2} \\
\hline u
\end{array}\right\}
\end{aligned}
$$

e

$$
\left\{\begin{array}{c|c}
\dot{x}_{r} \\
\hline-y_{r}
\end{array}\right\}=\left[\begin{array}{c|c}
A_{11} & B_{1} \\
\hline-C_{1} & C_{2} A_{22}^{-1} B_{2}
\end{array}\right]\left\{\begin{array}{c}
x_{r} \\
\hline u
\end{array}\right\}
$$

Como é conhecido que as matrizes $C$ e $D$ de um sistema $[A, B, C, D]$ não influenciam no comportamento dos estados do sistema, pode-se concluir por comparação entre as equações 216 e 218 que $x_{r}(t)=x_{1}(t)$. Portanto o erro $e(t)$ é

$$
e(t)=y_{1}(t)+y_{2}(t)-y_{r}(t)=y_{2}(t)+C_{2} A_{22}^{-1} B_{2} u(t)
$$


A partir da equação 215, pode-se calcular função de transferência do erro $G_{e}(s)$ :

$$
G_{e}=\sum_{k=1}^{n_{m}} G_{i}-\sum_{k=1}^{n_{m r}} G_{i}+D-D_{r}=C_{2}\left(s I-A_{22}\right)^{-1} B_{2}-C_{2} A_{22}^{-1} B_{2}
$$

Portanto pode-se simplificar e equação 215 de maneira que o erro do SR pode ser calculado como:

$$
\left\{\begin{array}{c|c}
\dot{x}_{2} \\
\hline e
\end{array}\right\}=\left[\begin{array}{c|c}
A_{22} & B_{2} \\
\hline C_{2} & C_{2} A_{22}^{-1} B_{2}
\end{array}\right]\left\{\begin{array}{c}
x_{2} \\
\hline u
\end{array}\right\}
$$

Como a resposta $e(t)$ encontrada pelas equações 215 e 220 é idêntica, a utilização da segunda é mais indicada por exigir menor custo computacional. Isso ocorre pois a ordem do sistema da equação 215 é maior que da equação 220

O erro da resposta estática do modelo reduzido também pode ser calculado a partir da equação 220, assumindo $\dot{x}_{2}=0$

$$
\left[\begin{array}{c|c}
A_{22} & 0 \\
\hline C_{2} & -I
\end{array}\right]\left\{\frac{x_{2 e q}}{e_{e q}}\right\}=\left\{\frac{-B_{2}}{-C_{2} A_{22}^{-1} B_{2}}\right\} u_{e q}
$$

Expandindo a equação 211, pode-se concluir que $e_{e q}=0$. Esse resultado já era previsto pois o foi utilizado método EPS para a eliminação de GDL, que garante que o erro da resposta estática seja nulo.

Baseado nas discussões apresentadas previamente neste trabalho, pode-se esperar as seguintes características para esta técnica:

- Como foi utilizado o método de eliminação por EPS, a resposta estática será igual à do SC.

- Como foi utilizado o método de eliminação EPS, o SR fornece uma boa aproximação da função de transferência do SC quando avaliado em baixas frequências pois $G(0)=G_{r}(0)$.

- Como $A_{11}=A_{r}$, as formas modais e os polos dos modos preservados no SR serão iguais aos do SC

- Caso o sistema completo seja estável, o modelo reduzido também será estável (pois preserva os polos do SC que são correspondentes aos modos mantidos).

- O vetor de estados do SR é idêntico aos estados preservados do SR: $x_{r}=x_{1}$.

- Esta técnica de redução é aplicável somente a sistemas cuja matriz $A_{22}$ seja invertível. 


\subsection{TÉCNICA R4}

Na técnica R4 foi realizada inicialmente uma mudança de base para a base de vetores singulares utilizando a equação 72 . A seleção de variáveis candidatas a serem eliminadas foi realizada pelo critério CSVS, descrito no item 6.2. O método de eliminação utilizado foi o de truncamento direto e não foi implementado nenhum método de correção da resposta do sistema reduzido.

Abaixo será apresentada uma discussão sobre o método de redução por truncamento direto aplicado à base de vetores singulares.

A partir da equação 104 o truncamento dos $r$ últimos GDL do sistema também pode ser interpretado como considerar nulos os $r$ últimos valores singulares da matriz $A$. $\mathrm{O}$ resultado disso é uma matriz $\widetilde{A}$ com a mesma dimensão de $A$ :

$$
\widetilde{A}=U_{v s}\left[\begin{array}{cccccc}
\sigma_{1} & & & & & \\
& \ddots & & & & \\
& & \sigma_{n_{r}} & & & \\
& & & 0 & & \\
& & & \ddots & \\
& & & & 0
\end{array}\right] V_{v s}^{*}
$$

Em (ZHOU; DOYLE, 1998), prova-se que a norma da diferença entre $A$ e $\widetilde{A}$ é igual ao maior valor singular que foi considerado nulo, ou seja, $\sigma_{n_{r}+1}$

$$
\|A-\widetilde{A}\|=\sigma_{n_{r}+1}
$$

Como o sistema formado por $\widetilde{A}$ contém valores singulares nulos, o núcleo (ou kernel) de $\widetilde{A}$ tem dimensão não nula. Isso faz com que o sistema não esteja em sua realização mínima.

Expandindo a equação 222 encontra-se a seguinte somatória, que é análoga à equação 69 truncada a partir dos termos de índice $n_{r}+1$

$$
A_{r}=\sum_{i=1}^{n_{r}} u_{i} \sigma_{i} v_{i}^{*}
$$

De maneira geral, a redução por truncamento direto é feita através das relações mostradas na equação 130 Entretanto, como método alternativo, matriz $A_{r}$ do SR na técnica R3 pode ser calculada através da equação 225

$$
A_{r}=U_{v s r}\left[\begin{array}{ccc}
\sigma_{1} & & \\
& \ddots & \\
& & \sigma_{n_{r}}
\end{array}\right] V_{v s r}^{*}
$$


onde $U_{v s r}=\left[u_{1}, u_{2}, \ldots, u_{n_{r}}\right]$ e $V_{v s r}=\left[v_{1}, v_{2}, \ldots, v_{n_{r}}\right]$.

Baseado nas discussões apresentadas previamente neste trabalho, pode-se esperar as seguintes características para esta técnica:

- A norma da diferença $\left\|A-A_{r}\right\|$ pode ser calculada com baixo custo computacional.

- Como foi utilizado o método de eliminação por truncamento direto, o SR fornece uma boa aproximação da função de transferência do SC quando avaliado em altas frequências $G(\infty)=G_{r}(\infty)$

- Mesmo que o SC seja estável, não há garantia de que o SR seja estável (ERSAL et al., 2008).

- Existe uma métrica que pode ser calculada antes da eliminação dos GDL, descrita na equação 223 Isso pode auxiliar na determinação do número de GDL a serem reduzidos, o que permite que sejam utilizados os processos na parte esquerda do fluxograma da figura 1 .

\subsection{TÉCNICA R5}

Na técnica R5 foi realizada inicialmente uma mudança de base para uma base de Krylov através do Método de Arnoldi, utilizando a equação 85. A seleção de variáveis candidatas a serem eliminadas foi realizada pelo critério CSVK, descrito no item 6.3 . O método de eliminação utilizado foi o de truncamento direto e não foi implementado nenhum método de correção da resposta do sistema reduzido.

Caso o sistema completo tenha apenas uma entrada, o vetor inicial utilizado para a base BKMA, utilizado na equacao 81 é igual à matriz $B$. Como consequência disso, os $n_{r}$ primeiros coeficientes de Markov $\bar{m}_{k}$ dos sistemas completo e reduzido são iguais (ANTOULAS; SORENSEN; GUGERCIN, 2001).

Outra característica importante sobre esta técnica é que mesmo que o SC seja estável, não há garantia de que o SR seja estável. Uma solução para evitar modelos reduzidos instáveis ou mal condicionados na base de Krylov é utilizar métodos implicitamente reiniciados (GRIMME; SORENSEN; DOOREN, 1996) e (JAIMOUKHA; KASENALLY, 1997).

Baseado nas discussões apresentadas previamente neste trabalho, pode-se esperar as seguintes características para esta técnica:

- Mesmo que o sistema completo seja estável, não há garantia de que o SR seja estável.

- Para sistemas com uma entrada, os $n_{r}$ primeiros coeficientes de Markov dos sistemas completo e reduzido são iguais. 


\subsection{TÉCNICA R6}

Na técnica R6 foi realizada inicialmente uma mudança de base para a base de realização balanceada, utilizando a equação 87. A seleção de variáveis candidatas a serem eliminadas foi realizada pelo critério baseado na controlabilidade e observabilidade, descrito no item 6.4. O método de eliminação utilizado foi o de truncamento direto e não foi implementado nenhum método de correção da resposta do SR. Diversos autores entitulam esta técnica como Truncamento Balanceado (ZHOU; DOYLE, 1998).

A norma $\mathcal{H}_{2}$ da função de transferência do erro $G_{e}(s)$ (ANTOULAS, 2005) pode ser calculada como:

$$
\left\|G_{e}\right\|_{\mathcal{H}_{2}}^{2}=\operatorname{tr}\left[\left(B_{2} B_{2}^{*}+2 Y_{2} A_{12}\right) \Gamma_{2}\right]
$$

Onde $Y_{1}$ e $Y_{2}$ são obtidos através da solução da equação de Sylvester:

$$
\left(\begin{array}{cc}
A_{11}^{*} & A_{21}^{*} \\
A_{12}^{*} & A_{22}^{*}
\end{array}\right)\left(\begin{array}{c}
Y_{1} \\
Y_{2}
\end{array}\right)+\left(\begin{array}{c}
Y_{1} \\
Y_{2}
\end{array}\right) A_{11}+\left(\begin{array}{c}
C_{1}^{*} \\
C_{2}^{*}
\end{array}\right) C_{1}=0
$$

O cálculo da norma $\left\|G_{e}\right\|_{\mathcal{H}_{2}}$ através da equação 226 é vantajoso em relação ao uso da equação 177 pois o custo computacional para a solução da equação 227 é menor que o da equação 7 (ANTOULAS, 2005). Isso pode ser percebido pois a dimensão da matriz $\left(Y_{1}^{T} Y_{2}^{T}\right)^{T}$ é menor que a dimensão dos gramianos de controlabilidade ou observabilidade.

Glover provou que pode-se estabelecer um limite superior para a norma $\mathcal{H}_{\infty}$ da função de transferência do erro $G_{e}(s)$ : (GLOVER, 1984)

$$
\left\|G_{e}\right\|_{\mathcal{H}_{\infty}} \leq 2 \sum_{n_{r}+1}^{n} \gamma_{i}
$$

Muscato (MUSCATO, 2000) mostra que modelos reduzidos são assintoticamente estáveis, estão em sua realização mínima e respeitam a inequação 228 quando respeitam as seguintes condições:

- O sistema completo é assintoticamente estável

- O sistema completo está projetado na base de realização balanceada.

- O critério de seleção utilizado é o CSNH.

- A eliminação de GDL é feita por qualquer um dos seguintes métodos: truncagem direta, EPS, EPSG e EPSGP. 
- Nenhuma metodologia de correção de resposta aplicada.

A igualdade na inequação 228 é verdadeira quando $\gamma_{n_{r}+1}=\cdots=\gamma_{n}$ (ANTOULAS, 2005).

Baseado nas discussões apresentadas previamente neste trabalho, pode-se esperar as seguintes características para esta técnica:

- A norma $\mathcal{H}_{2}$ pode ser calculada com menor custo computacional em relação ao método geral descrito na equação 177 .

- O limite superior da norma $\left\|G_{e}\right\|_{\mathcal{H}_{\infty}}$ pode ser calculado com baixo custo computacional na etapa de seleção dos GDL a serem eliminados (antes que o SR seja propriamente calculado).

- Caso o sistema completo seja estável, o modelo reduzido também será estável (PERNEBO; SILVERMAN, 1982).

- Como foi utilizado o método de eliminação por truncamento direto, o SR fornece uma boa aproximação da função de transferência do SC quando avaliado em altas frequências $G(\infty)=G_{r}(\infty)$

- O limite superior da norma $\left\|G_{e}\right\|_{\mathcal{H}_{\infty}}$ pode ser calculado antes da eliminação dos GDL. Isso pode auxiliar na determinação do número de GDL a serem reduzidos, o que permite que sejam utilizados os processos na parte esquerda do fluxograma da figura 1

\subsection{TÉCNICA R7}

Na técnica R7 foi realizada inicialmente uma mudança de base para a base de realização balanceada descrita no item 5.4. A seleção de variáveis candidatas a serem eliminadas foi realizada pelo critério baseado na controlabilidade e observabilidade, descrito no item 6.4. 0 método de eliminação utilizado foi o de Eliminação por Perturbação Singular (EPS), descrito no item 7.3.1 e não foi implementado nenhum método de correção da resposta do sistema reduzido.

Quando aplicado na base de realização balanceada, o método EPS preserva a estabilidade do sistema após a eliminação das variáveis selecionadas (LIU; ANDERSON, 1989).

Baseado nas discussões apresentadas previamente neste trabalho, pode-se esperar as seguintes características para esta técnica:

- Caso o sistema completo seja estável, o modelo reduzido também será estável. 
- O limite superior da norma $\left\|G_{e}\right\|_{\mathcal{H}_{\infty}}$ pode ser calculado com baixo custo computacional na etapa de seleção dos GDL a serem eliminados (antes que o SR seja propriamente calculado).

- Como foi utilizado o método de eliminação por EPS, o SR fornece uma boa aproximação da função de transferência do SC quando avaliado em baixas frequências (LIU; ANDERSON, 1989). Además, a resposta estática será igual à do $\mathrm{SC} G(0)=G_{r}(0)$.

- O limite superior da norma $\left\|G_{e}\right\|_{\mathcal{H}_{\infty}}$ pode ser calculado antes da eliminação dos GDL. Isso pode auxiliar na determinação do número de GDL a serem reduzidos, o que permite que sejam utilizados os processos na parte esquerda do fluxograma da figura 1

- Esta técnica de redução é aplicável somente a sistemas cuja matriz $A_{22}$ seja invertível.

\subsection{TÉCNICA R8}

Na denominada técnica R8 foi realizada inicialmente uma mudança de base para a BFM utilizando a equação 43. A seleção de GDL candidatos a serem eliminados foi realizada pelo critério CSCO, descrito no item 6.1.3. O método de eliminação utilizado foi o de truncamento direto e foi implementada a metodologia de correção apresentada no item 8.1.1.

A metodologia de correção foi implementade de maneira que a resposta da função de transferência do modelo reduzido seja idêntica à do modelo completo em $s_{0}=0$.

Após a eliminação dos GDL, o sistema não corrigido pode ser descrito pela equação 208 . onde $A_{r}=A_{11}, B_{r}=B_{1}, C_{r}=C_{1}$ e $D=0$.

Utilizando a equação 154 e adotando $s_{0}=0$, encontra-se a matriz $D_{r c}$ do sistema corrigido.

$$
D_{r c}=C_{2} A_{22}^{-1} B_{2}
$$

Portanto, de acordo com as informações apresentadas no item 8.1.1. o modelo reduzido e corrigido será formado pelas matrizes $\left[A_{r}, B_{r}, C_{r}, D_{r c}\right]$ e possui erro nulo $G_{e}(0)=0$.

$$
\left\{\begin{array}{c|c}
\dot{x}_{r} \\
\hline y_{r}
\end{array}\right\}=\left[\begin{array}{c|c}
A_{11} & B_{1} \\
\hline C_{1} & C_{2} A_{22}^{-1} B_{2}
\end{array}\right]\left\{\begin{array}{c}
x_{r} \\
\hline u
\end{array}\right\}
$$

Pode-se perceber que o modelo reduzido através da técnica R8 é idêntico ao modelo encontrado pela técnica R3. Portanto os resultados referentes à técnica R8 serão omitidos do capítulo 12 e serão apenas mostrados resultados referentes à técnica R3. 


\section{RESULTADOS}

Neste capítulo serão apresentados e analisados os resultados de modelos reduzidos segundo a metodologia de redução descrita no capítulo 4.

Inicialmente no item 12.1 serão mostradas características do modelo completo de veículo de passeio, descrito no item 10.1. No item 12.2, serão implementados os critérios de seleção de GDL descritos no capítulo 6 .

No item 12.3 são mostrados resultados dos modelos reduzidos a partir do modelo completo veículo de passeio. Estes modelos foram obtidos utilizando as técnicas de redução descritas no capítulo 11 e elencadas na tabela 5 . A respresentatividade total dos modelos reduzidos é calculada e usada como critério de comparação de desempenho entre eles.

A influência do critério de seleção de GDL utilizado no processo de redução é discutida no item 12.4. Para tal estudo, foi utilizado o modelo de veículo de passeio descrito no item 10.1 .

O modelo completo de trem foi utilizado para um estudo sobre a influência do número de GDL eliminados na resposta do sistema reduzido, apresentado no item 12.5

\subsection{PROPRIEDADES DO MODELO COMPLETO DE VEÍCULO DE PASSEIO}

O veículo completo de passeio, conforme descrito no item 10.2 possui polos conforme a figura 20 .

Figura 20 - Diagrama de polos e zeros do sistema

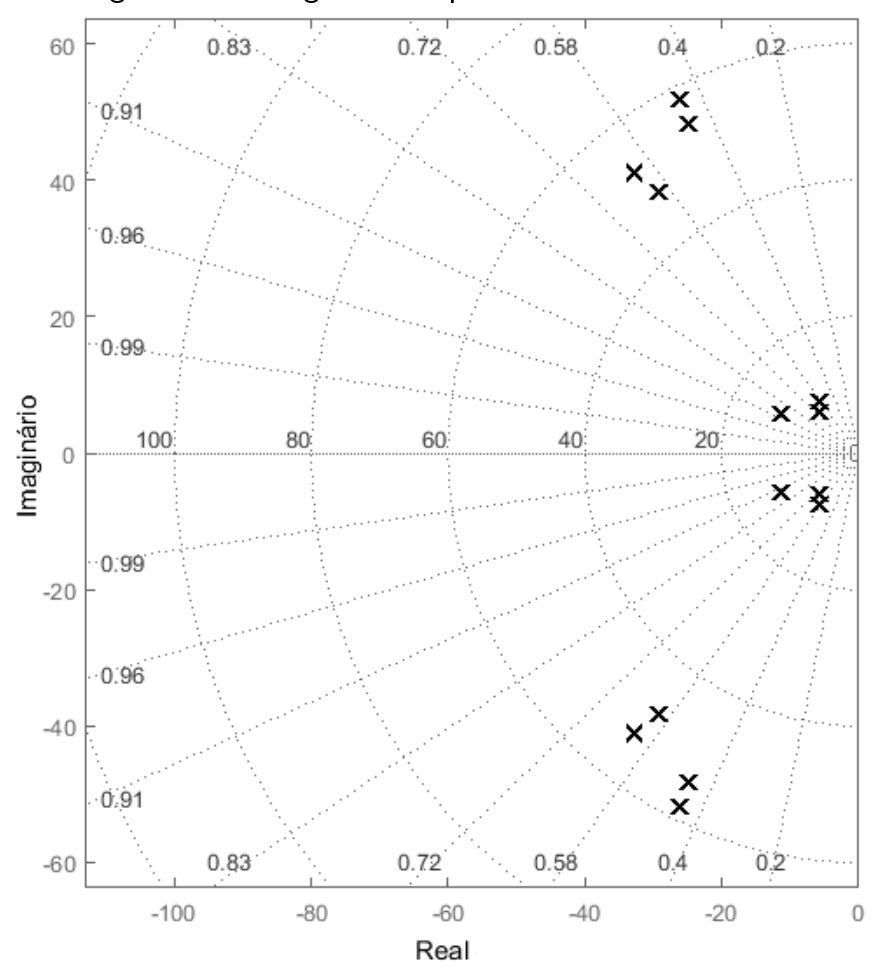


A tabela 6 mostra os pólos do sistema organizados de maneira que a frequência natural esteja em ordem crescente. Observa-se que o sistema completo é assintoticamente estável pois possui todos os polos com parte real negativa.

Tabela 6 - Localização dos polos do sistema

\begin{tabular}{|c|c|c|c|c|}
\hline $\begin{array}{c}\text { Número do } \\
\text { modo de vibrar }\end{array}$ & $\begin{array}{c}\text { Parte } \\
\text { real }\end{array}$ & $\begin{array}{c}\text { Parte } \\
\text { imaginária }\end{array}$ & $\begin{array}{c}\text { Frequência } \\
\omega_{n}(\mathrm{~Hz})\end{array}$ & $\begin{array}{c}\text { Fator de } \\
\text { amortecimento } \zeta\end{array}$ \\
\hline 1 & $-5,60$ & $\pm 6,07$ & 1,31 & 0,678 \\
\hline 2 & $-5,67$ & $\pm 7,50$ & 1,50 & 0,603 \\
\hline 3 & $-11,2$ & $\pm 5,63$ & 2,00 & 0,894 \\
\hline 4 & $-29,2$ & $\pm 38,2$ & 7,66 & 0,607 \\
\hline 5 & $-32,7$ & $\pm 41,2$ & 8,37 & 0,621 \\
\hline 6 & $-24,8$ & $\pm 48,3$ & 8,64 & 0,458 \\
\hline 7 & $-26,0$ & $\pm 52,0$ & 9,25 & 0,448 \\
\hline
\end{tabular}

Como exemplo, as figuras 21 a 24 mostram as formas modais correspondentes aos polos mostrados tabela 6. As formas modais, que são as partes real e imaginária do autovetor complexo (indicadas respectivamente como $u_{k}$ e $v_{k}$ na equação 38) estão nas cores em azul e vermelha respectivamente. A configuração de equilíbrio do veículo está indicada na cor preta e o centro de massa indicado pelo símbolo $\oplus$.

Figura 21 - Formas modais do primeiro (esquerda) e segundo (direita) modos de vibrar do SC

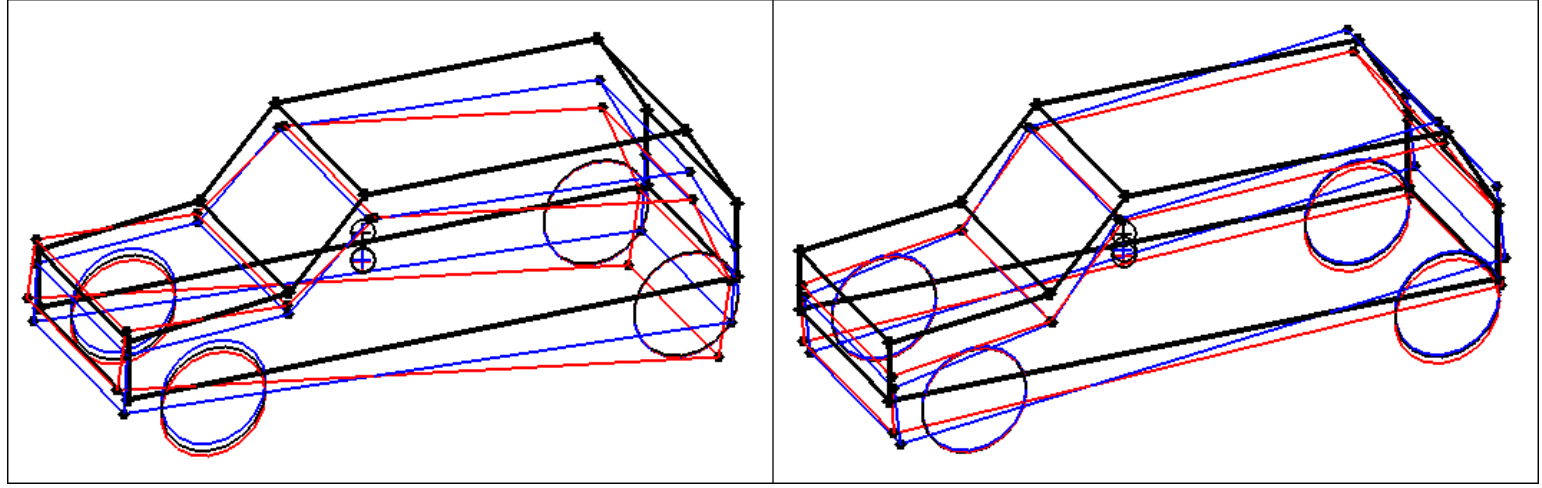


Figura 22 - Formas modais do terceiro (esquerda) modo de vibrar do SC

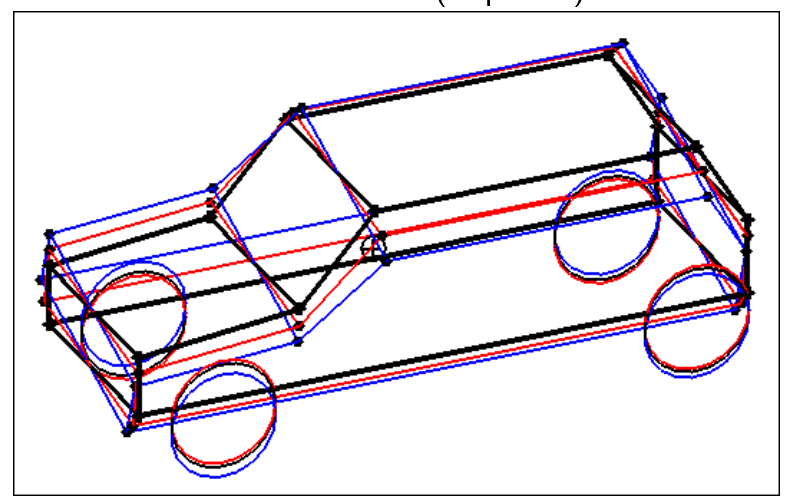

Figura 23 - Formas modais do quarto (esquerda) e quinto (direita) modos de vibrar do SC

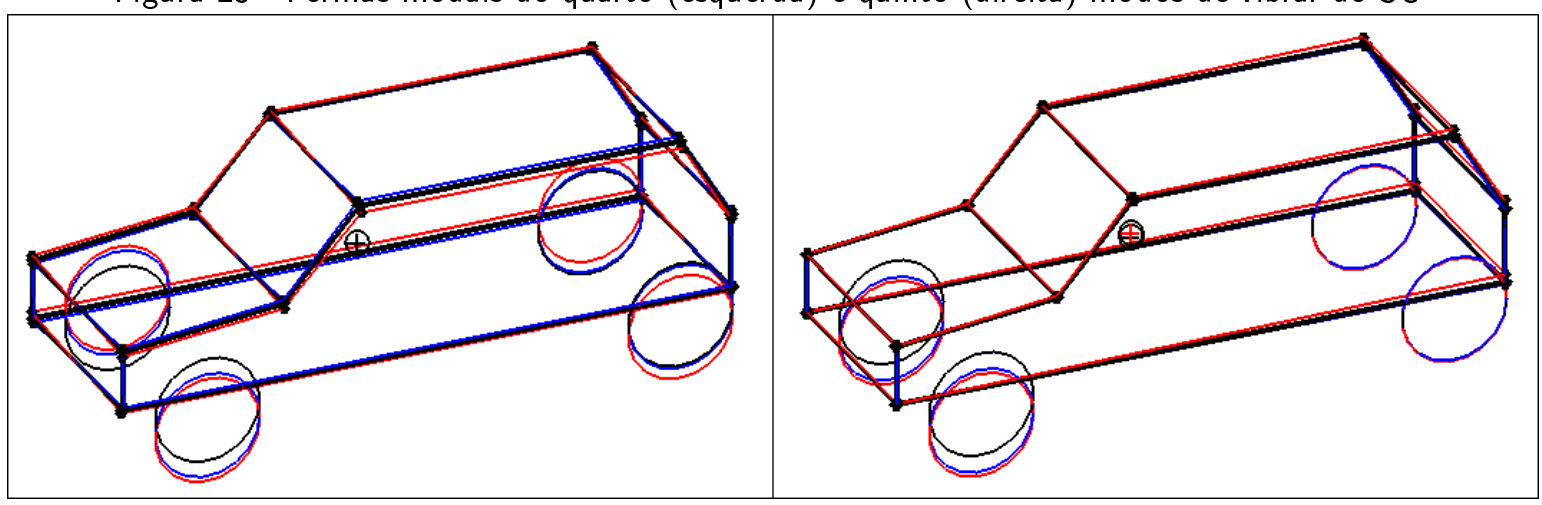

Figura 24 - Formas modais do sexto (esquerda) e sétimo (direita) modos de vibrar do SC

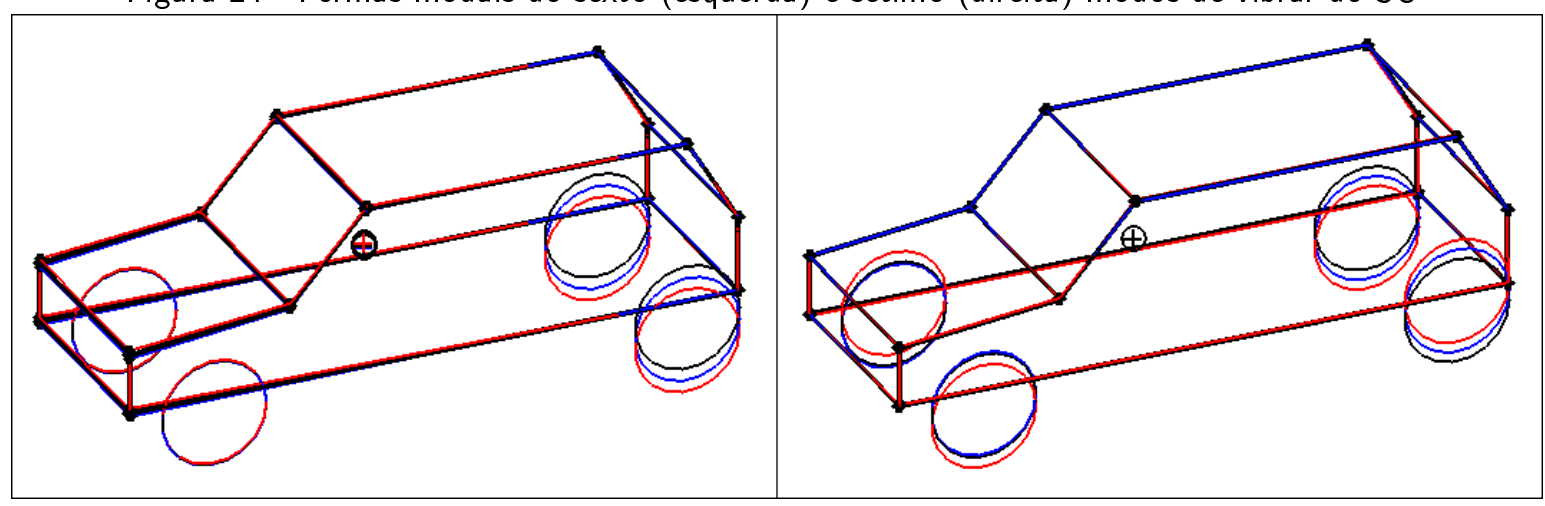




\subsection{RESULTADOS DOS CRITÉRIOS DE SELEÇÃO DE GDL}

Nos itens 12.2.1 a 12.2.6, serão implementados no modelo completo de veículo de passeio os critérios de seleção descritos no capítulo 6 .

Os parâmetros de seleção foram calculados para cada critério tal que valores menores destes parâmetros correspondem a GDL que são bons candidatos à eliminação.

Os itens 12.2.1 a 12.2.4 se referem aos critérios de seleção que são apliváveis apenas em sistemas projetados na BFM.

No item 12.2.5 será implementado o critério CSVS, aplicável a sistemas projetados na base de vetores singulares. No item 12.2.6 será implementado o critério CSNH, aplicável a sistemas projetados na base de balanceada.

Como o critério de seleção CSVK para a base BKMA é baseado na ordem dos vetores da base e não em um parâmetro específico, não há resultados a serem apresentados para este critério de seleção.

\subsubsection{Seleção de GDL segundo o critério CSF}

A tabela 7 mostra os valores do parâmetro de seleção $P_{\omega_{n}}$ para cada modo de vibrar, de acordo com o item 6.1.1. Este parâmetro é igual ao inverso da frequência natural $\omega_{n k}$ (em radianos por segundo) do k-ésimo modo de vibrar, conforme a equação 97 . Os modos foram organizados de maneira que o parâmetro de seleção esteja em ordem decrescente, portanto os modos com menores valores de $P_{\omega_{n}}$ são bons candidatos à eliminação.

Tabela 7 - Parâmetro de seleção $P_{\omega_{n}}$ baseado no critério CSF
\begin{tabular}{|c|c|c|}
\hline $\begin{array}{c}\text { Relevância do } \\
\text { modo de vibrar }\end{array}$ & $\begin{array}{c}\text { Número do } \\
\text { modo de vibrar }\end{array}$ & $\begin{array}{c}\text { Valor do parâmetro } \\
\text { de seleção } P_{\omega_{n}}\end{array}$ \\
\hline 1 & 1 & 0,1212 \\
\hline 2 & 2 & 0,1063 \\
\hline 3 & 3 & 0,0795 \\
\hline 4 & 4 & 0,0208 \\
\hline 5 & 5 & 0,0190 \\
\hline 6 & 6 & 0,0184 \\
\hline 7 & 7 & 0,0172 \\
\hline
\end{tabular}

A figura 26 mostra uma representação gráfica do critério de seleção CSF. Nela pode-se ver dois grupos indicados por áreas na cor cinza. Os polos com maiores valores do parâmetro de 
Figura 25 - Parâmetro de seleção $P_{\omega_{n}}$ baseado no critério CSF

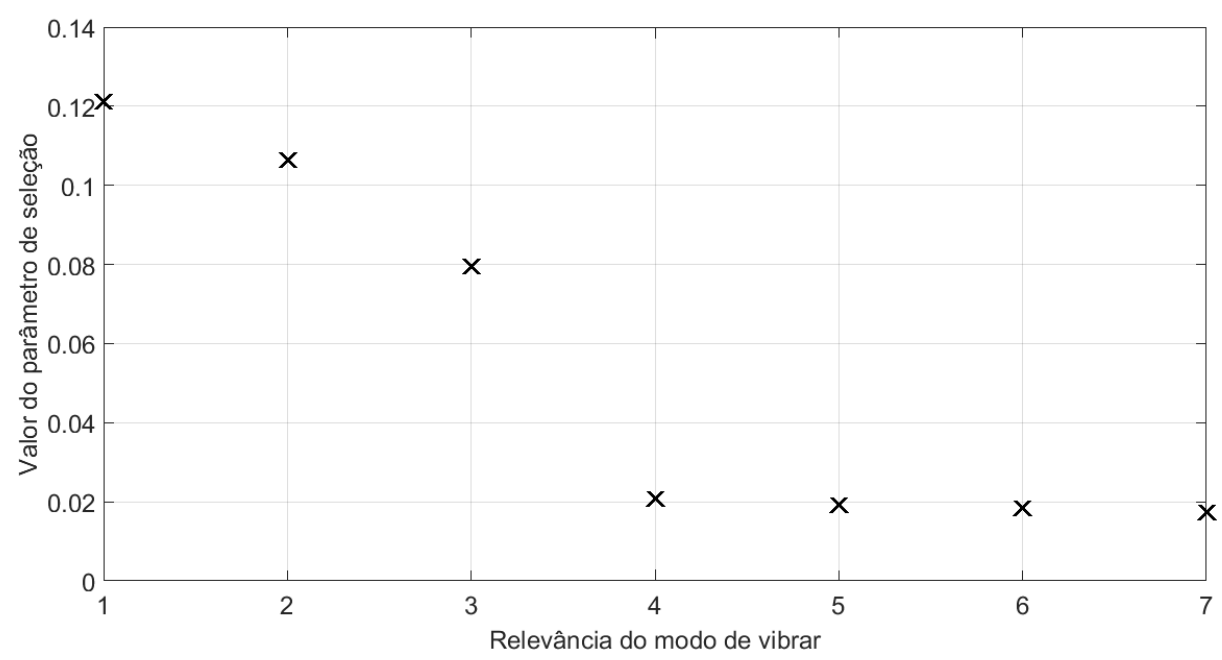

Figura 26 - Representação gráfica dos modos mais e menos dominantes segundo critério CSF

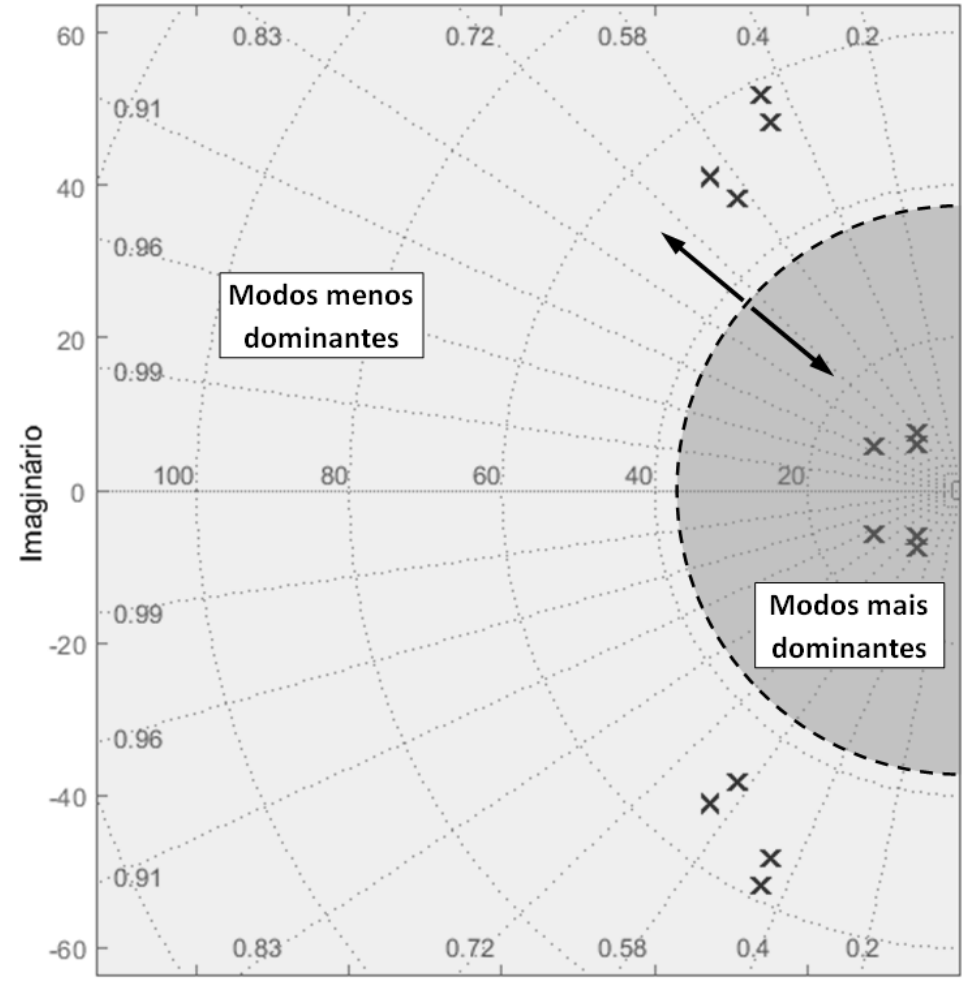

seleção e consequentemente bons candidatos a serem preservados, estão indicados no circulo mais central. Já os bons candidatos a serem eliminados, estão indicados pela área mais externa.

\subsubsection{Seleção de GDL segundo o critério CSFA}

A tabela 8 mostra os valores do parâmetro de seleção $P_{a f}$ para cada modo de vibrar, de acordo com o item 6.1.2. Este parâmetro é igual ao inverso do produto da frequência natural 
$\omega_{n k}$ (em radianos por segundo) pelo fator de amortecimento $\zeta_{k}$ do mesmo modo, conforme equação 98 . Os modos foram organizados de maneira que o parâmetro de seleção esteja em ordem decrescente, portanto os modos com menores valores de $P_{a f}$ são bons candidatos à eliminação.

Tabela 8 - Parâmetro de seleção $P_{a f}$ baseado no critério CSFA

\begin{tabular}{|c|c|c|}
\hline $\begin{array}{c}\text { Relevância do } \\
\text { modo de vibrar }\end{array}$ & $\begin{array}{c}\text { Número do } \\
\text { modo de vibrar }\end{array}$ & $\begin{array}{c}\text { Valor do parâmetro } \\
\text { de seleção } P_{a f}\end{array}$ \\
\hline 1 & 1 & 0,1787 \\
\hline 2 & 2 & 0,1764 \\
\hline 3 & 3 & 0,0889 \\
\hline 4 & 6 & 0,0403 \\
\hline 5 & 7 & 0,0384 \\
\hline 6 & 4 & 0,0342 \\
\hline 7 & 5 & 0,0306 \\
\hline
\end{tabular}

Figura 27 - Parâmetro de seleção $P_{a f}$ baseado no critério CSFA

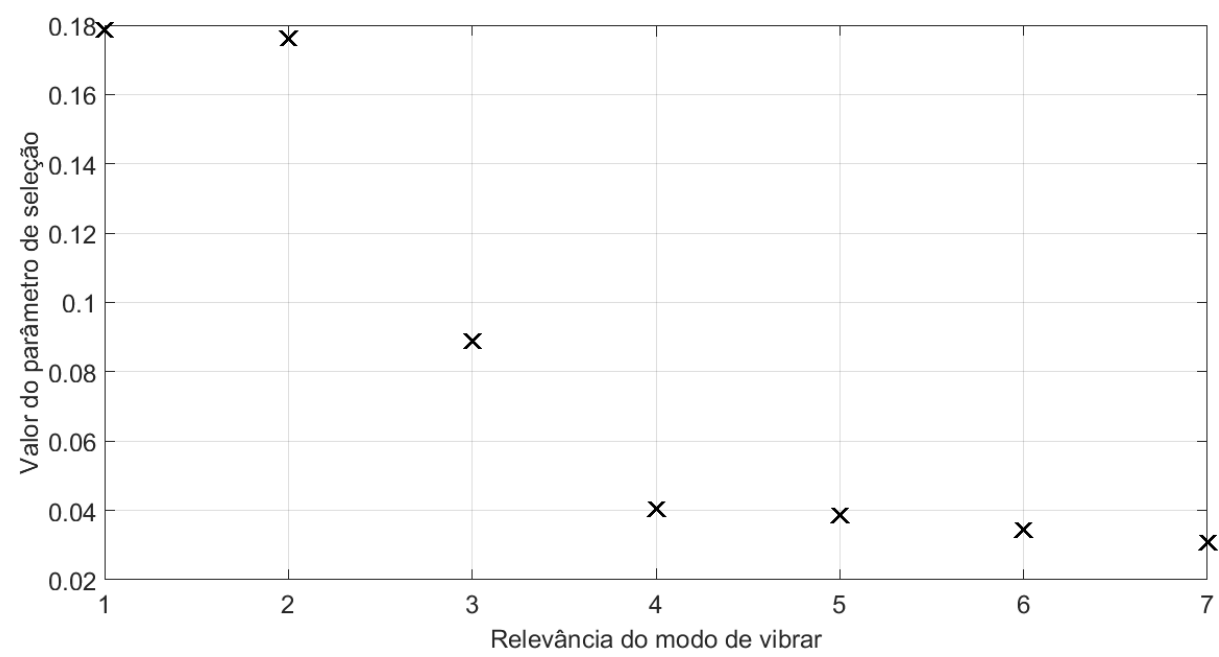

A figura 28 mostra uma representação gráfica do critério de seleção CSFA. Nela pode-se ver dois grupos indicados por áreas na cor cinza. Os polos que obtiveram maiores valores do parâmetro de seleção e consequentemente são bons candidatos a serem preservados, estão indicados na área à direita. Já os bons candidatos a serem eliminados, estão indicados pela área mais à esquerda. 
Figura 28 - Representação gráfica dos modos mais e menos dominantes segundo critério CSFA

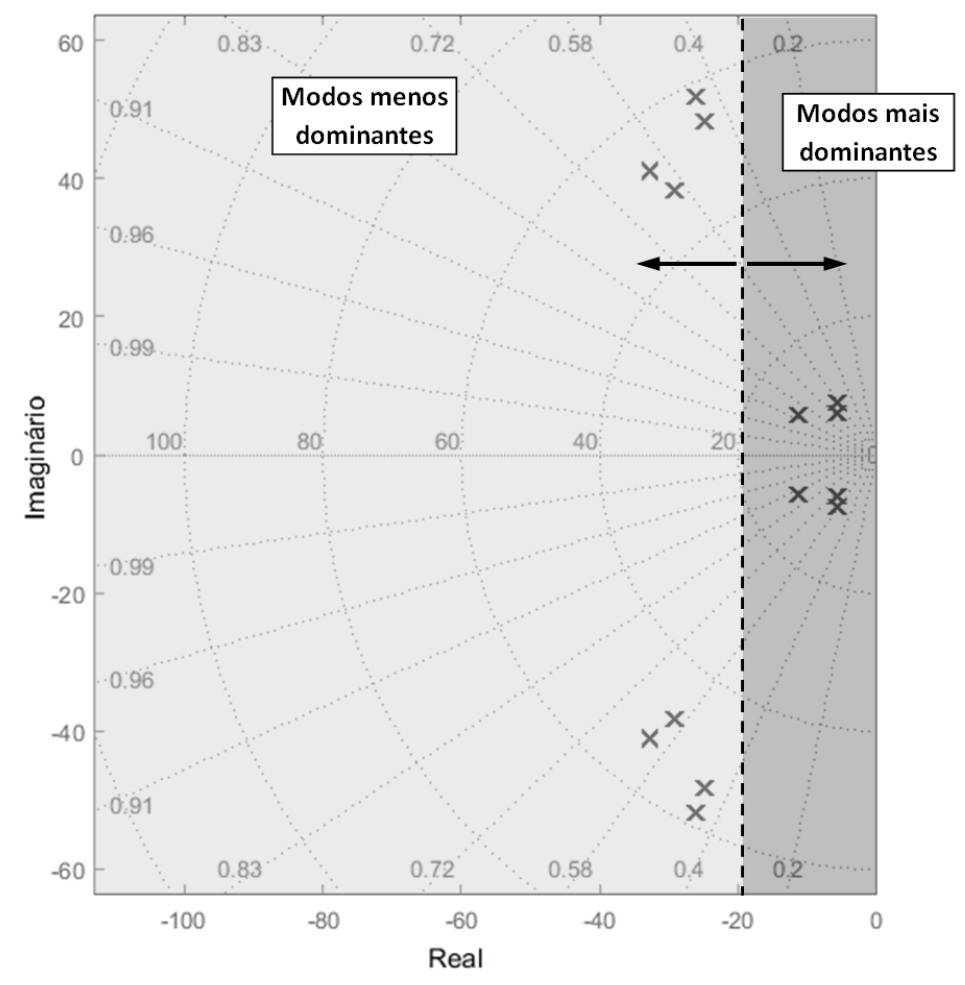

\subsubsection{Seleção de GDL segundo o critério CSCO}

A tabela 9 mostra as normas Hankel dos sistemas que descrevem a dinâmica dos modos de vibrar do sistema completo. Estes sistemas podem ser representados como na equação 45 .

Como o sistema completo em questão possui pares de autovalores complexos conjugados e distintos, cada um dos sistemas desacoplados que descrevem a dinâmica dos modos de vibrar tem ordem 2 e portanto duas normas Hankel cada. As normas Hankel dos modos de vibrar foram organizadas sendo $\gamma_{1}$ e $\gamma_{2}$ respectivamente as normas de maior e menor valor de cada modo.

A figura 29 mostra a comparação entre as normas Hankel do sistema completo e dos modos de vibrar. Os resultados mostram que as normas Hankel dos modos de vibrar têm valores próximos aos das normas Hankel do sistema completo de veículo de passeio.

A tabela 10 mostra os valores do parâmetro de seleção $P_{\gamma m}$ para cada modo de vibrar, conforme a equação 100 . Nela, pode-se observar a taxa de decrescimento dos valores do critério de seleção, facilitando a visualização e a determinação do grupo de modos menos dominantes, que serão eliminados no processo de redução.

Pode-se perceber que os valores da terceira coluna da tabela 10 são iguais ao máximo valor entre $\gamma_{1}$ e $\gamma_{2}$, conforme a equação 100 . 
Tabela 9 - Normas Hankel dos modos de vibrar

\begin{tabular}{|c|c|c|}
\hline $\begin{array}{c}\text { Número do } \\
\text { modo de vibrar }\end{array}$ & $\begin{array}{c}\text { Primeira norma Hankel } \\
\text { do modo }\left(\gamma_{1}\right)\end{array}$ & $\begin{array}{c}\text { Segunda norma Hankel } \\
\text { do modo }\left(\gamma_{2}\right)\end{array}$ \\
\hline 1 & 0,5898 & 0,1254 \\
\hline 2 & 0,5036 & 0,1479 \\
\hline 3 & 0,3800 & 0,1671 \\
\hline 4 & 0,2834 & $0,927110^{-1}$ \\
\hline 5 & $0,631410^{-1}$ & $0,258110^{-1}$ \\
\hline 6 & $0,781010^{-1}$ & $0,354910^{-1}$ \\
\hline 7 & 0,1067 & $0,267410^{-1}$ \\
\hline
\end{tabular}

Figura 29 - Comparação entre as normas Hankel do sistema completo e dos modos de vibrar

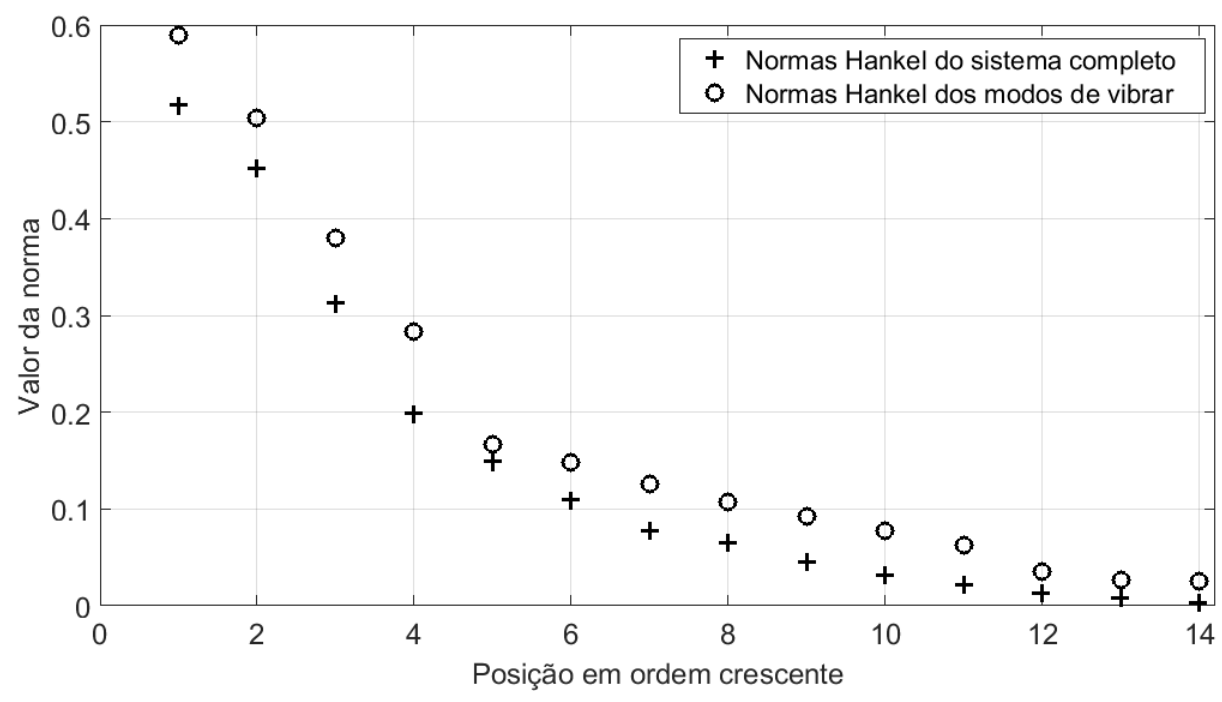

Figura 30 - Parâmetro de seleção $P_{\gamma m}$ baseado no critério CSCO

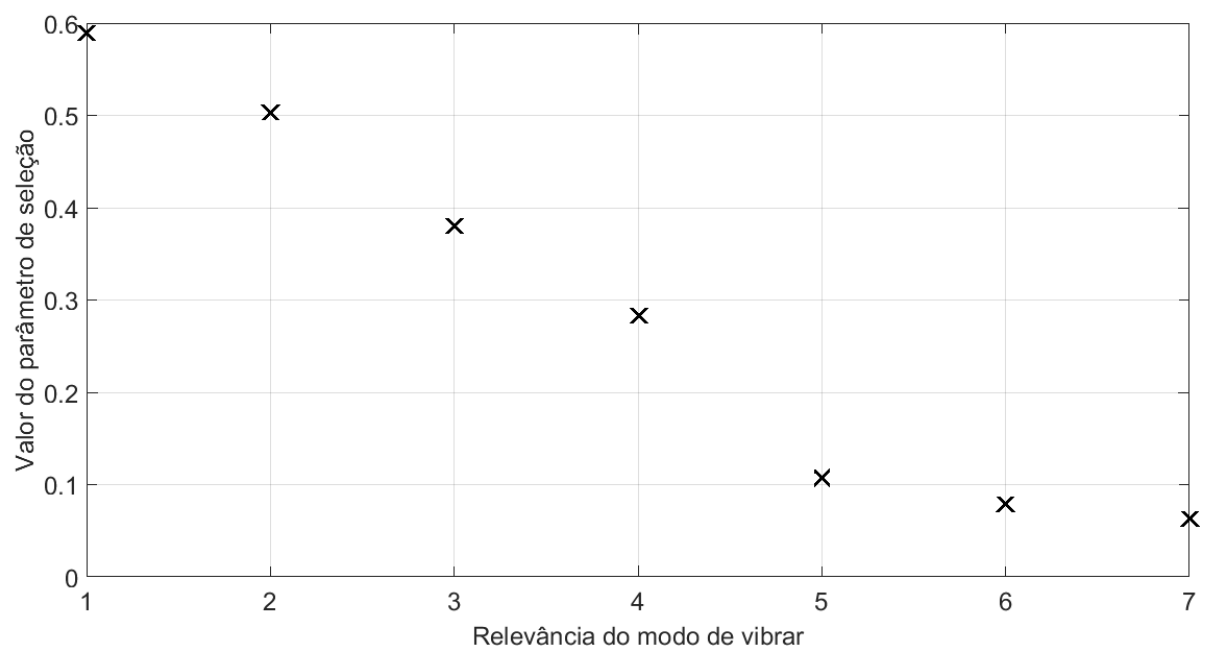


Tabela 10 - Parâmetro de seleção $P_{\gamma m}$ baseado no critério CSCO

\begin{tabular}{|c|c|c|}
\hline $\begin{array}{c}\text { Relevância do } \\
\text { modo de vibrar }\end{array}$ & $\begin{array}{c}\text { Número do } \\
\text { modo de vibrar }\end{array}$ & $\begin{array}{c}\text { Valor do parâmetro } \\
\text { de seleção } P_{\gamma m}\end{array}$ \\
\hline 1 & 3 & 0,5898 \\
\hline 2 & 1 & 0,5036 \\
\hline 3 & 2 & 0,3800 \\
\hline 4 & 4 & 0,2834 \\
\hline 5 & 5 & 0,1067 \\
\hline 6 & 6 & $0,781010^{-1}$ \\
\hline 7 & 7 & $0,631310^{-1}$ \\
\hline
\end{tabular}

\subsubsection{Seleção de GDL segundo o critério CSFT}

A tabela 11 mostra os valores do parâmetro de seleção $P_{s}$ para cada modo de vibrar, de acordo com o item 6.1.4. Para o cálculo do parâmetro $P_{s}$ foi usada a frequência de interesse $s_{0}=6,5 \mathrm{~Hz}$ definida arbitrariamente. Este parâmetro é igual ao inverso do maior valor singular da função de transferência na frequência $s_{0}$, conforme descrito na equação 103 .

Figura 31 - Máximo valor singular da função de transferência dos modos de vibrar do sistema completo

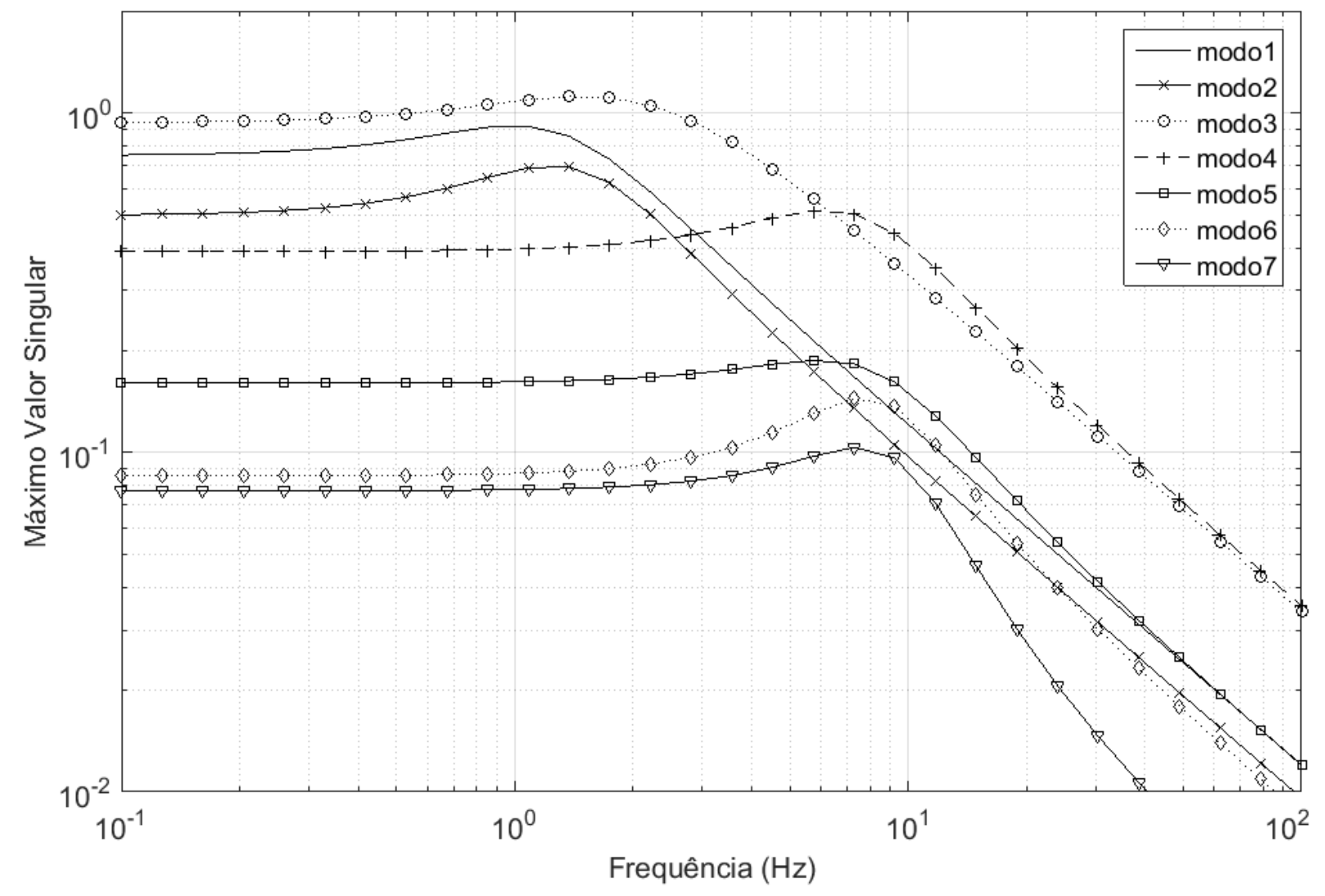


Tabela 11 - Parâmetro de seleção $P_{s}$ baseado no critério CSFT

\begin{tabular}{|c|c|c|}
\hline $\begin{array}{c}\text { Relevância do } \\
\text { modo de vibrar }\end{array}$ & $\begin{array}{c}\text { Número do } \\
\text { modo de vibrar }\end{array}$ & $\begin{array}{c}\text { Valor do parâmetro de } \\
\text { seleção } P_{s} \text { em } 6,5 \mathrm{~Hz}\end{array}$ \\
\hline 1 & 4 & 0,5167 \\
\hline 2 & 3 & 0,5000 \\
\hline 3 & 1 & 0,1879 \\
\hline 4 & 5 & 0,1863 \\
\hline 5 & 2 & 0,1521 \\
\hline 6 & 6 & 0,1387 \\
\hline 7 & 7 & 0,1013 \\
\hline
\end{tabular}

A figura 31 mostra as funções de transferência $G_{k}(s)$ dos modos de vibrar do sistema completo.

Na figura 31 observa-se que:

- Para $s_{0}=1 \mathrm{~Hz}$, a ordem dos modos segundo o critério CSFT é: 3,1,2,4,5,6,7 (do modo mais dominante para o menos dominante).

- Para $s_{0}=4 \mathrm{~Hz}$, a ordem dos modos segundo o critério CSFT é: 3,4,1,2,5,6,7 (do modo mais dominante para o menos dominante).

Figura 32 - Parâmetro de seleção $P_{s}$ baseado no critério CSFT

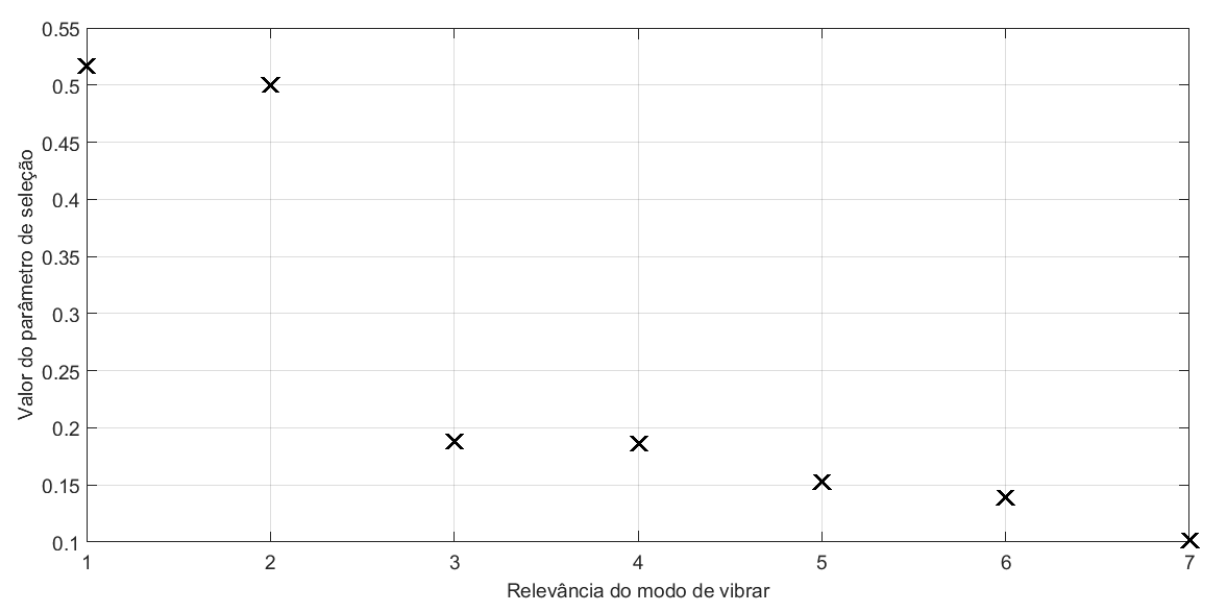

Portanto a frequência de interesse $s_{0}$ tem forte influência na seleção dos modos que são bons candidatos a serem preservados ou eliminados pelo critério CSFT.

A figura 32 mostra graficamente os valores de $P_{s}$ atribuídos a cada modo de vibrar. Os modos foram organizados de maneira que o parâmetro de seleção esteja em ordem decrescente, portanto os modos com menores valores de $P_{s}$ são bons candidatos à eliminação. 


\subsubsection{Seleção de GDL segundo o critério CSVS}

Utilizando a mudança para a base de vetores singulares descrita no item 5.2 foram calculados o valores do parâmetro de seleção $P_{\sigma}$ para cada GDL, conforme a equação 105 . Os valores deste parâmetro são iguais aos valores singulares da matriz $A$ do sistema completo e estão organizados em ordem decrescente na tabela 12 .

Figura 33 - Parâmetro de seleção $P_{\sigma}$ baseado no critério CSVS

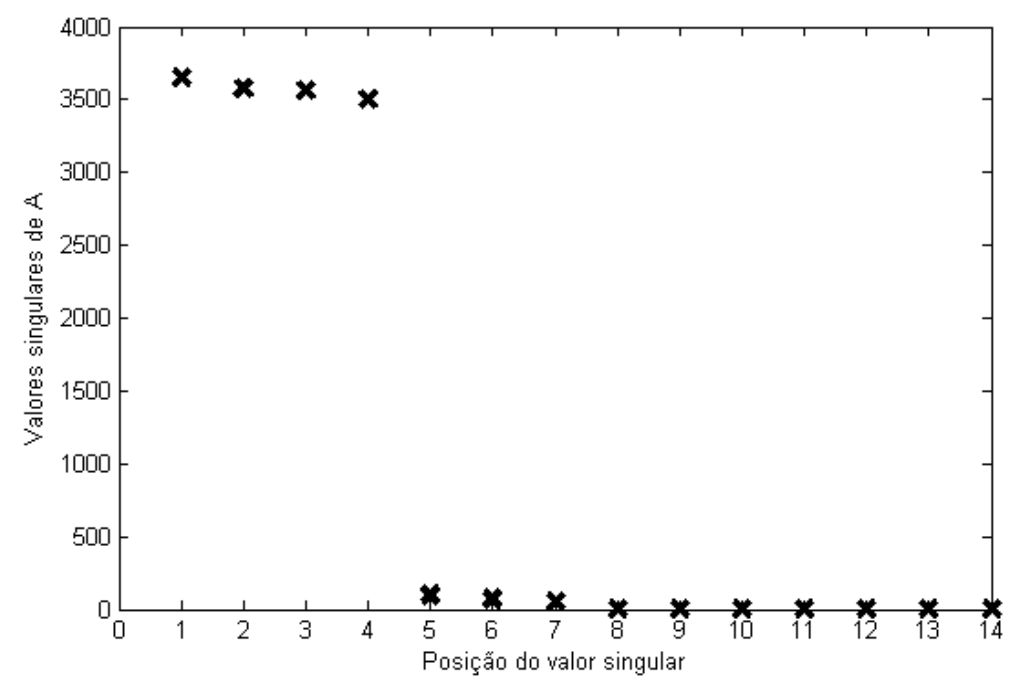

\subsubsection{Seleção de GDL segundo o critério CSNH}

Utilizando a mudança para a base balanceada descrita no item 5.4, foram calculados os parâmetros de seleção $P_{\gamma}$ para cada GDL, conforme a equação 106 . Estes valores são iguais às normas Hankel do sistema completo e estão organizados em ordem decrescente na tabela 13. 
Tabela 12 - Parâmetro de seleção $P_{\sigma}$ baseado no critério CSVS

\begin{tabular}{|c|c|}
\hline $\begin{array}{c}\text { Relevância } \\
\text { do GDL }\end{array}$ & $\begin{array}{c}\text { Valor do parâmetro } \\
\text { de seleção } P_{\sigma}\end{array}$ \\
\hline 1 & $3,651510^{3}$ \\
\hline 2 & $3,576110^{3}$ \\
\hline 3 & $3,567310^{3}$ \\
\hline 4 & $3,510810^{3}$ \\
\hline 5 & $1,006310^{2}$ \\
\hline 6 & $7,482210^{1}$ \\
\hline 7 & $5,191010^{1}$ \\
\hline 8 & 1,0000 \\
\hline 9 & 1,0000 \\
\hline 10 & 1,0000 \\
\hline 11 & 0,9999 \\
\hline 12 & 0,9887 \\
\hline 13 & 0,9822 \\
\hline 14 & 0,9780 \\
\hline
\end{tabular}

Figura 34 - Parâmetro de seleção $P_{\gamma}$ baseado no critério CSNH

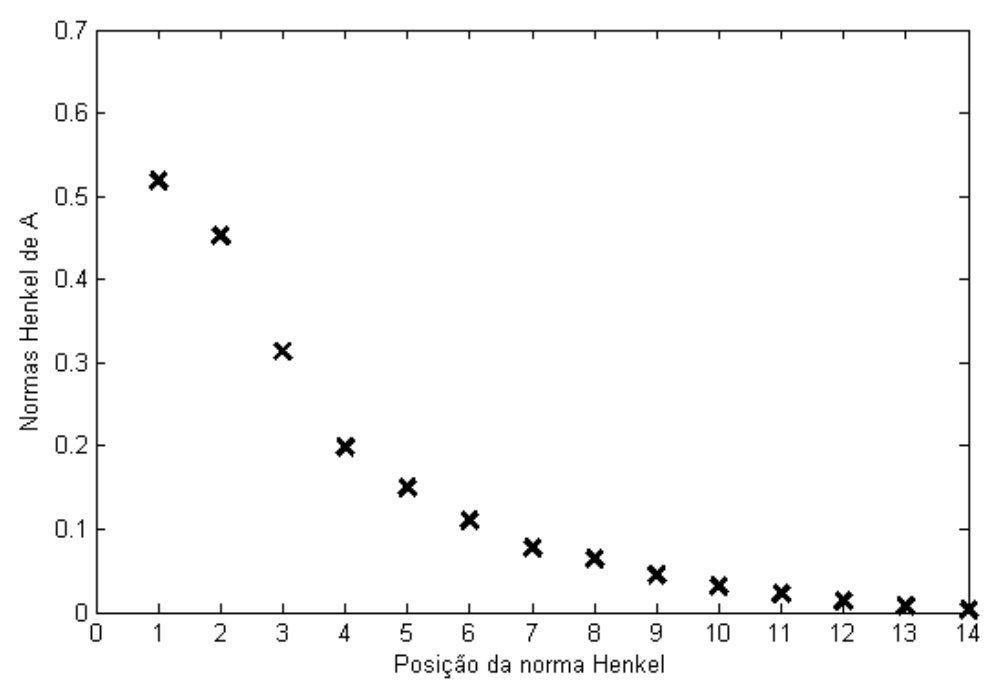


Tabela 13 - Parâmetro de seleção $P_{\gamma}$ baseado no critério CSNH

\begin{tabular}{|c|c|}
\hline $\begin{array}{c}\text { Relevância } \\
\text { do GDL }\end{array}$ & $\begin{array}{c}\text { Valor do parâmetro } \\
\text { de seleção } P_{\gamma}\end{array}$ \\
\hline 1 & 0,5180 \\
\hline 2 & 0,4520 \\
\hline 3 & 0,3134 \\
\hline 4 & 0,1989 \\
\hline 5 & 0,1499 \\
\hline 6 & 0,1099 \\
\hline 7 & $0,772910^{-1}$ \\
\hline 8 & $0,644910^{-1}$ \\
\hline 9 & $0,451810^{-1}$ \\
\hline 10 & $0,313410^{-1}$ \\
\hline 11 & $0,219010^{-1}$ \\
\hline 12 & $0,131010^{-1}$ \\
\hline 13 & $0,801210^{-2}$ \\
\hline 14 & $0,298510^{-2}$ \\
\hline
\end{tabular}




\subsection{RESULTADOS COMPARATIVOS}

Neste item, serão comparados os resultados dos modelos reduzidos pelas técnicas descritas no capítulo 11. Estes foram calculados a partir do modelo completo de veículo de passeio descrito no item 10.1 .

Como comentado no item 11.8, os resultados do modelo reduzido pela técnica R8 serão omitidos pois são idênticos aos do modelo reduzido pela técnica R3.

No item 12.3.2 serão apresentados os resultados no domínio do tempo, para uma entrada degrau. Posteriormente no item 12.3.3 serão apresentados resultados dos modelos no domínio da frequência.

No item 12.3.4 serão apresentadas métricas de representatividade calculadas para cada modelo reduzido.

No item 12.3.5, a representatividade total $\mathcal{R}$ será calculada para cada um dos modelos reduzidos e usada como critério de desempenho para a comparação entre eles.

\subsubsection{Cálculo do número de GDL eliminados}

Para modelos reduzidos pela técnica R2, a norma $\left\|G_{e}\right\|_{\mathcal{H}_{\infty}}$ pode ser aproximada pela maior norma $\left\|G_{k}\right\|_{\mathcal{H}_{\infty}}$ dentre os modos de vibração eliminados, segundo a equação 212 . Como mostrado no capítulo 11 esta relação pode ser extendida para modelos reduzidos por truncamento modal e para qualquer critério de seleção desde que não seja aplicado nenhum método de correção.

A tabela 14 mostra a norma $\left\|G_{k}\right\|_{\mathcal{H}_{\infty}}$ de cada modo de vibrar do sistema completo de veículo de passeio. Os modos estão organizados segundo o critério de seleção CSCO, utilizado na técnica R2.

Tabela 14 - Norma $\left\|G_{k}\right\|_{\mathcal{H}_{\infty}}$ dos modos de vibrar do modelo completo

\begin{tabular}{|c|c|c|}
\hline $\begin{array}{c}\text { Relevância do modo } \\
\text { segundo o critério CSCO }\end{array}$ & $\begin{array}{c}\text { Número do } \\
\text { modo de vibrar }\end{array}$ & $\begin{array}{c}\text { Valor da } \\
\text { norma }\left\|G_{k}\right\|_{\mathcal{H}_{\infty}}\end{array}$ \\
\hline 1 & 3 & 1,1198 \\
\hline 2 & 1 & 0,9172 \\
\hline 3 & 2 & 0,6991 \\
\hline 4 & 4 & 0,5174 \\
\hline 5 & 5 & $\mathbf{0 , 1 8 5 5}$ \\
\hline 6 & 6 & 0,1454 \\
\hline 7 & 7 & 0,1035 \\
\hline
\end{tabular}


Calculando a norma $\mathcal{H}_{\infty}$ do sistema completo, obtém-se $\|G\|_{\mathcal{H}_{\infty}}=0,747$. Escolhendo que o erro entre as normas $\mathcal{H}_{\infty}$ do sistema completo e reduzido seja menor que $33 \%$, o máximo valor admissível para a norma $\mathcal{H}_{\infty}$ do erro é $\left\|G_{e}\right\|_{\mathcal{H}_{\infty}} \leq 0,25$.

Como o valor máximo admissível para a norma $\left\|G_{e}\right\|_{\mathcal{H}_{\infty}}$ é 0,25 , o número de modos a serem eliminados é igual a 3 para o modelo MR2, segundo a equação 212 . Consequentemente, a estimativa para o valor de $\left\|G_{e}\right\|_{\mathcal{H}_{\infty}}$ é igual à norma $\mathcal{H}_{\infty}$ do quinto modo do modelo completo segundo a ordem determinada pelo critério CSCO.

$$
\left\|G_{e}\right\|_{\mathcal{H}_{\infty}} \cong\left\|G_{k=5}\right\|_{\mathcal{H}_{\infty}}=0,1855
$$

Para modelos reduzidos pelas técnicas R6 ou R7, a norma $\left\|G_{e}\right\|_{\mathcal{H}_{\infty}}$ é menor ou igual à soma das normas Hankel correspondentes aos GDL eliminados, segundo a equação 228.

Como o valor máximo admissivel para a norma $\left\|G_{e}\right\|_{\mathcal{H}_{\infty}}$ é 0,25 , foram somados os valores das normas Hankel (mostrados na tabela 13) dos GDL menos dominantes até que não fosse ultrapassado o valor limite acima estipulado.

Realizando o procedimento acima, o número de GDL a serem eliminados é 6 e a soma das normas Hankel dos GDL eliminados é mostrada na equação 232. Este limite superior é válido para os modelos MR6 e MR7 com 6 GDL eliminados.

$$
\left\|G_{e}\right\|_{\mathcal{H}_{\infty}} \leq 2 \sum_{i=9}^{14} \gamma_{i}=0,245
$$

Portanto espera-se que os modelos MR2, MR3, MR6 e MR7 aproximem a norma $\mathcal{H}_{\infty}$ do modelo completo com erro inferior a 33\%.

Para o modelo MR4, a norma da diferença entre as matrizes $A$ e $\tilde{A}$ é igual ao maior valor singular correspondente aos GDL eliminados, segundo a equação 223 Portanto é desejável que os valores singulares correspondentes aos GDL eliminados sejam pequenos em relação aos dos GDL mantidos.

Pode-se visualizar ao lado direito da figura 33, um grupo de 10 valores singulares com valor pequeno em comparação aos demais. Portanto recomenda-se que número de GDL eliminados seja menor ou igual a 10 , pois a norma descrita na equação 223 será consideravelmente maior caso sejam truncados mais que $10 \mathrm{GDL}$. Para que os modelos reduzidos sejam comparados sob as mesmas condições, a técnica R4 foi implementada com a redução de 6 GDL. Neste caso, a norma calculada pela equação 223 é

$$
\|A-\widetilde{A}\|=\sigma_{9}=1,0
$$

O autor salienta a vantagem em se utilizar as técnicas de redução R2, R4, R6 e R7 pois possibilitam que o analista possa estimar o número de GDL a serem eliminados antes que seja 
calculado o modelo reduzido.

Para que os resultados sejam comparados sob as mesmas condições, as técnicas R1, R3 e R5 foram implementadas com a redução de $6 \mathrm{GDL}$. Desta meneira todos os modelos reduzidos foram obtidos com a eliminação da mesma quantidade de GDL.

No modelo MR1, os GDL que foram selecionados para eliminação segundo o conhecimento prévio do autor são: $Z_{4}, \dot{Z}_{4}, Z_{3}, \dot{Z}_{3}, Z_{2}$ e $\dot{Z}_{2}$.

Vale a pena destacar que o modelo reduzido pela técnica R1 com eliminação de 8 ou mais GDL possui sua matriz $B_{r}$ nula. Portanto nestas condições, o modelo se torna não controlável.

No modelo MR5, o vetor inicial para a base BKMA utilizado na equação 81, foi inicialmente determinado como a primeira coluna da matriz $B$ (ANTOULAS; SORENSEN; GUGERCIN, 2001). Entretanto, o número de condicionamento da matriz $A_{r}$ do sistema reduzido é $\mathcal{C}=$ $3 \cdot 10^{17}$, revelando que o sistema é mal condicionado. A possibilidade deste comportamento nos modelos reduzidos utilizando bases do tipo BKMA é descrita no mesmo trabalho por Antoulas et. al..

$\mathrm{O}$ número de condicionamento de uma matriz é definido como $\mathcal{C}=\|A\|\left\|A^{-1}\right\|$, que é equivalente a $\mathcal{C}=\sigma_{\max }(A) \sigma_{\min }(A)$ (STRANG, 2006).

Para contornar o problema supracitado, a base BKMA foi novamente calculada com o vetor inicial arbitrariamente escolhido como a parte real do primeiro autovetor da matriz $A$ (que corresponde ao autovalor de maior módulo). O número de condicionamento da matriz $A_{r}$ do novo sistema reduzido é $\mathcal{C}=23.7$ e foi considerado aceitável pelo autor. Portanto neste item serão apresentadas as respostas referentes ao segundo modelo reduzido, que possui matriz $A_{r}$ que é bem condicionada.

O autor avaliou a estabilidade dos sete modelos reduzidos que serão analisados neste item. Os modelos são assintoticamente estáveis e, para fins de objetividade, os dados que comprovam esta característica foram omitidos pois não correspondem ao escopo principal deste trabalho.

\subsubsection{Resultados no domínio do tempo}

Os resultados das figuras 35 a 38 correspondem a uma entrada degrau de amplitude 0,1 metro no pneu dianteiro.

$$
\left\{\begin{array}{l}
u(t)=[0 ; 0 ; 0 ; 0] \quad \text { para } t<0 \\
u(t)=[0,1 ; 0 ; 0 ; 0] \quad \text { para } t \geq 0
\end{array}\right.
$$

A equação 4 foi utilizada para calcular o erro $e(t)$ de cada modelo reduzido. A figura 35 mostra a componente $e_{2}(t)$ do vetor $e(t)$, que representa o erro do ângulo de rolagem, para entrada degrau. 
Figura 35 - Ângulo de rolagem para entrada degrau de $100 \mathrm{~mm}$

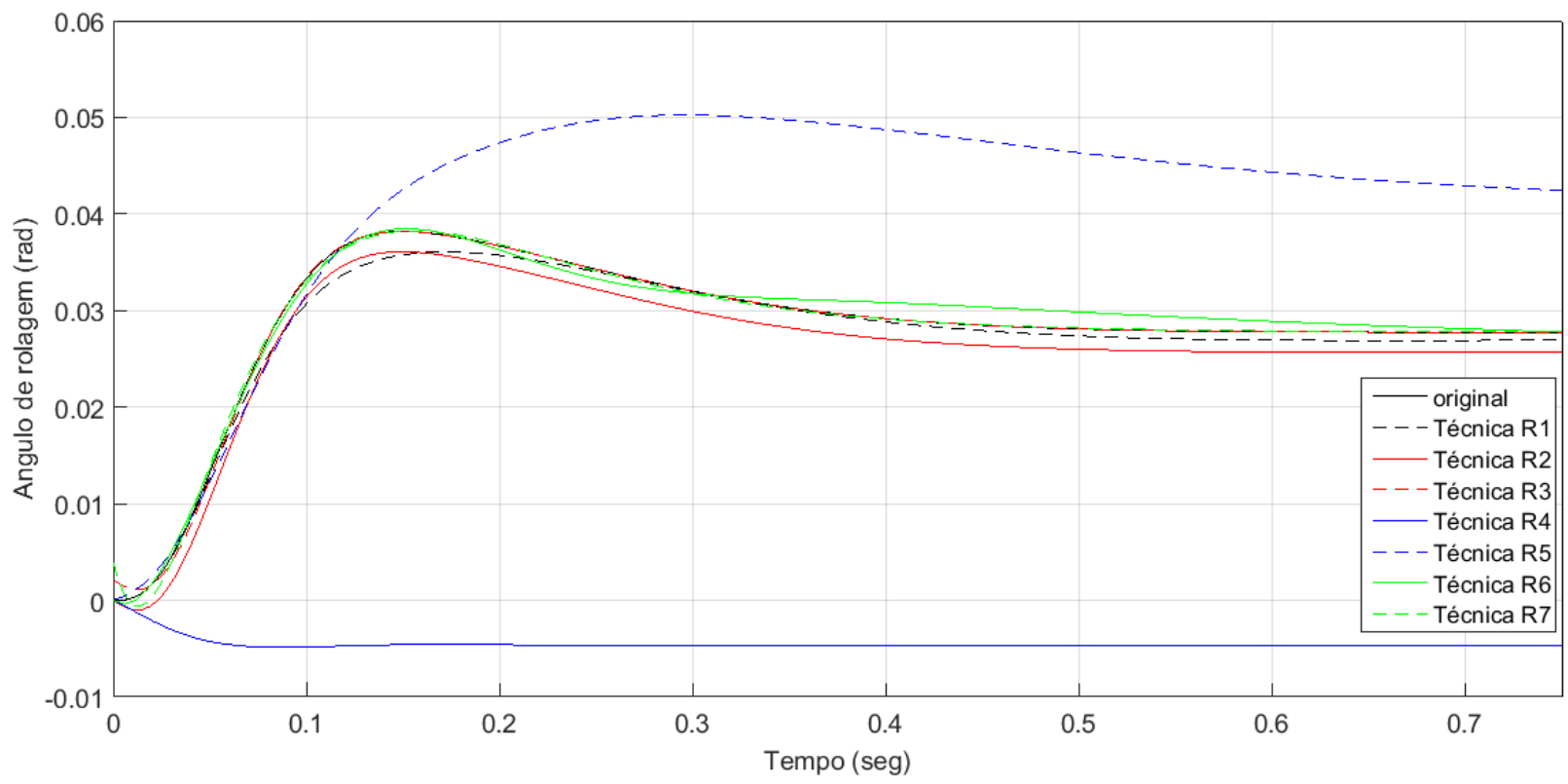

Figura 36 - Erro do ângulo de rolagem para entrada degrau de 100mm

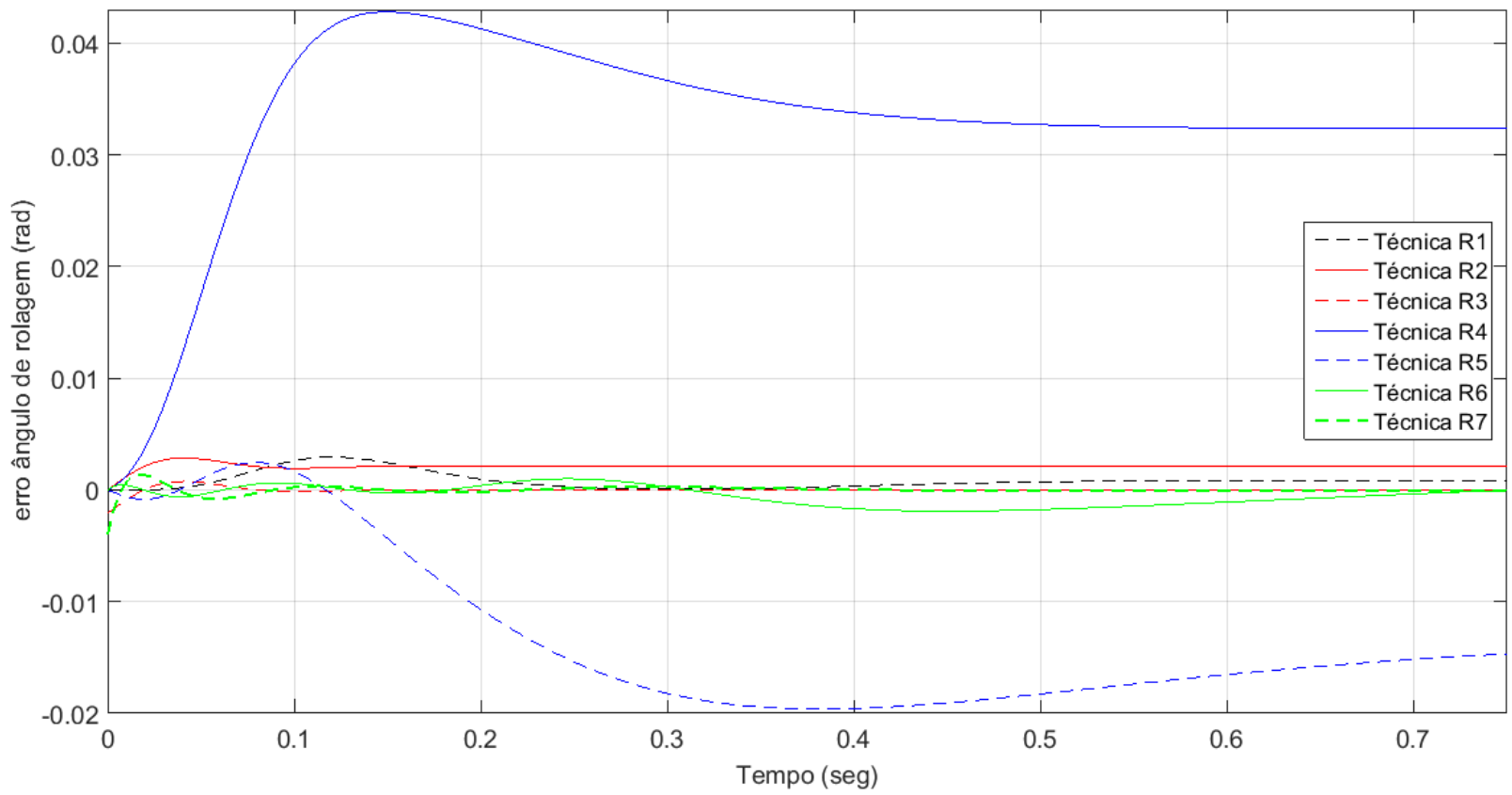

Com a 35 observa-se que os modelos reduzidos pelas técnicas R1, R2, R3, R6 e R7 obtiveram uma boa aproximação para o ângulo de rolagem na simulação de entrada degrau.

Como o vetor de respostas do sistema $y(t)$ é de dimensão $n_{y}=3$, a comparação entre os sistemas pode ser dificultada pela quantidade de informação gerada. Para contornar esta 
situação, o erro quadrático $e_{q}(t)$ de cada modelo reduzido foi calculado. A figura 37 mostra o erro quadrátido calculado segundo a equação 165 .

Figura 37 - Erro quadrático para entrada degrau de 100mm

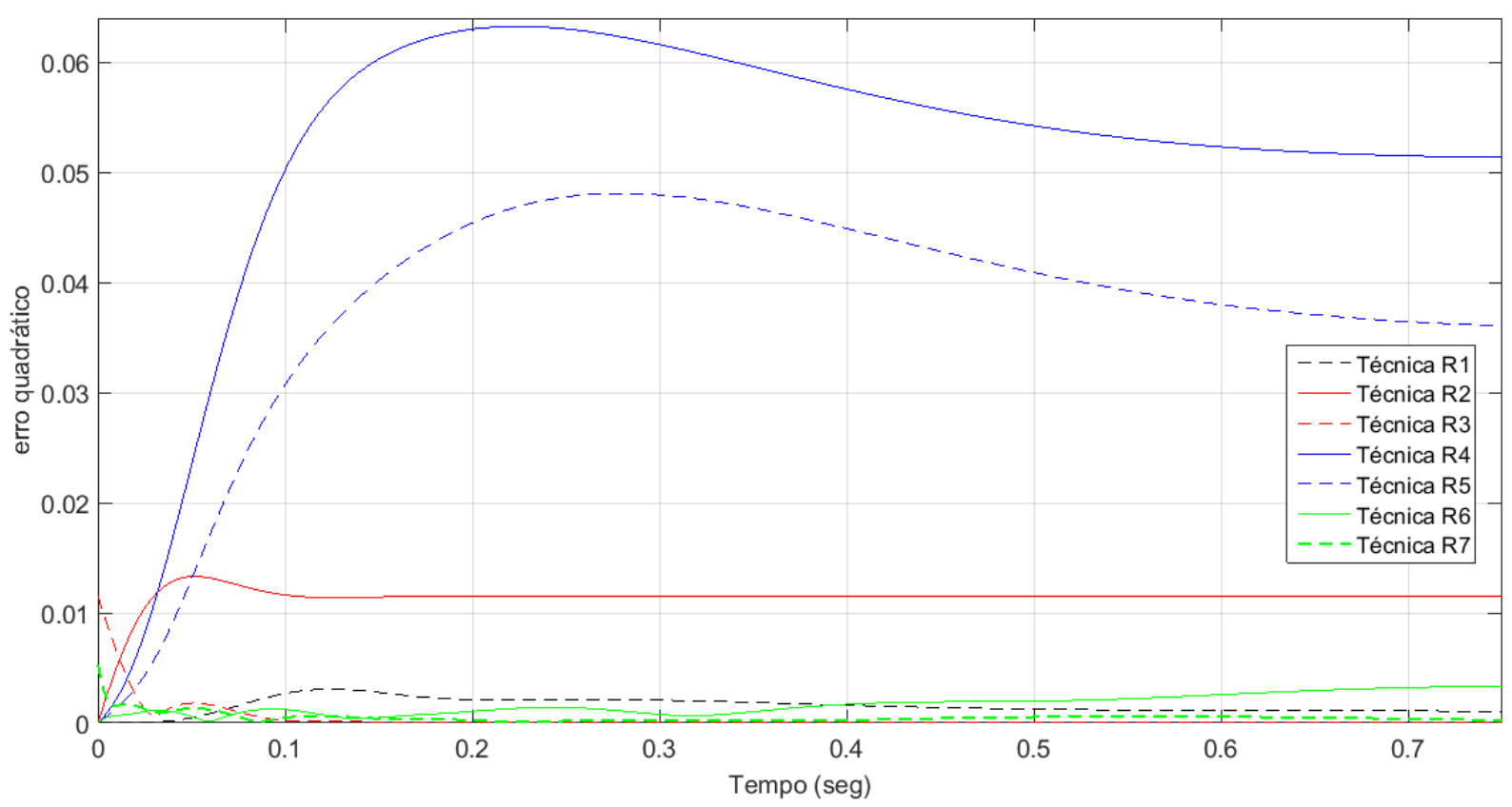

Para melhor visualização do comportamento dos modelos, a figura 38 mostra o erro quadrático $e_{q}(t)$ em escala logarítimica em função do tempo.

Figura 38 - Erro quadrático em escala logarítmica para entrada degrau de $100 \mathrm{~mm}$

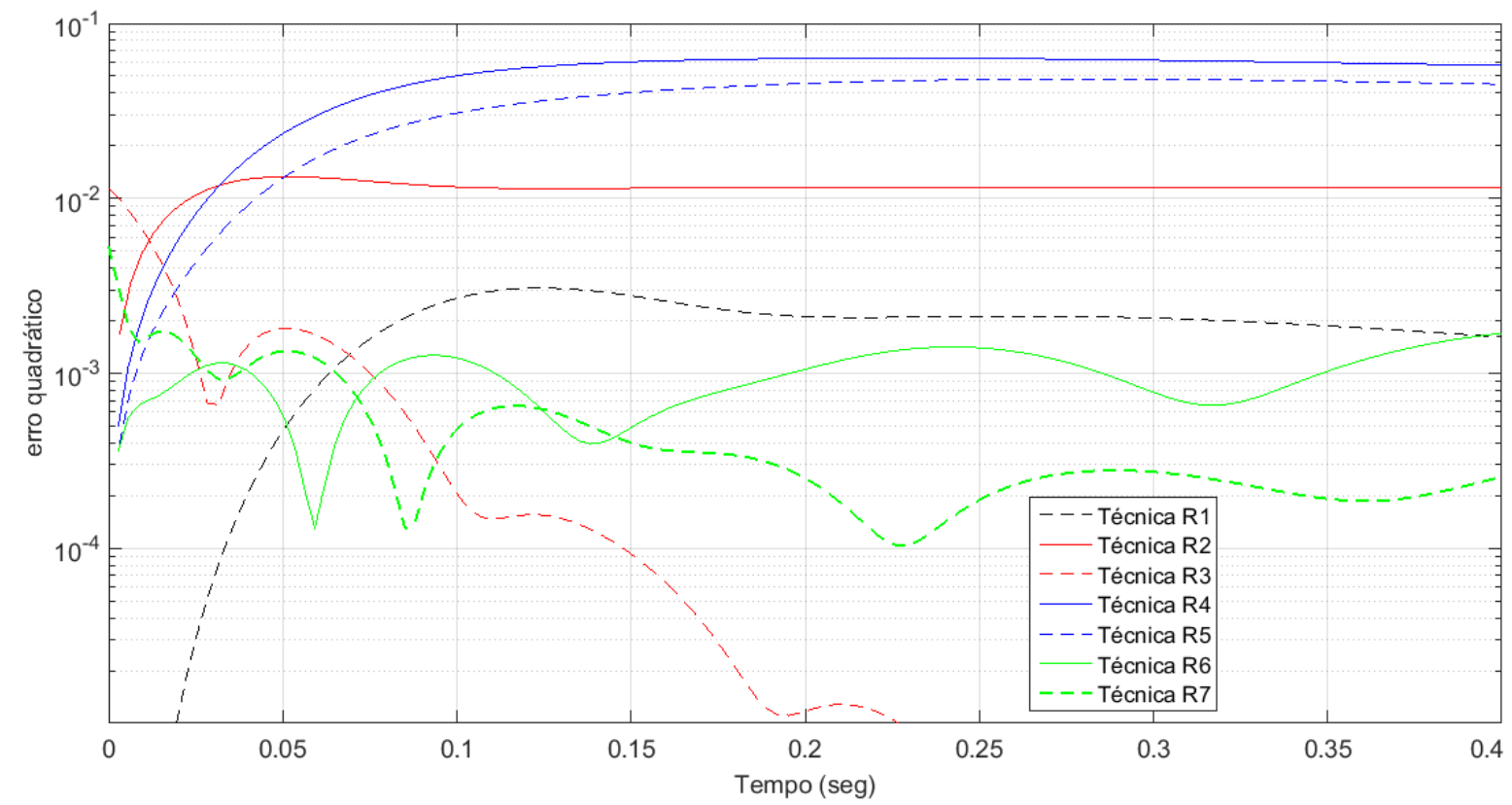

Nas figuras 36, 37 e 38 observa-se que o erro dos modelos R3 e R7 é não nulo no instante 
$t=0$. Isso é consequência do fato de que suas respectivas matrizes $D_{e}=D-D_{r}$ têm componentes não nulos, isso faz com que exista resposta instantânea para uma entrada $u(t)$ qualquer.

Na figura 37 observa-se que o erro dos modelos R3 e R7 diminui tendendo a zero em $t \rightarrow \infty$ (o que pode ser constatado na figura 47). Este comportamento foi previsto no capítulo 11 e é consequência da utilização do método de eliminação EPS.

\subsubsection{Resultados no domínio da frequência}

Os resultados apresentados nas figuras 39 e 40 correspondem ao comportamento dos modelos reduzidos no domínio da frequência, sob uma excitação vertical $u(t)$ de amplitude unitária no pneu dianteiro esquerdo.

$$
u(t)=\operatorname{sen}(\omega t)[1 ; 0 ; 0 ; 0]^{T}
$$

A figura 39 mostra o diagrama de Bode do ângulo de rolagem do veículo. Nela observa-se que o ângulo de fase da resposta dos modelos reduzidos é próxima à do modelo completo em frequências abaixo de $0,1 \mathrm{~Hz}$. Entretanto em frequências maiores que $10 \mathrm{~Hz}$, a fase da resposta os modelos reduzidos apresentam maior discrepância em relação ao modelo completo.

Figura 39 - Diagrama de bode para ângulo de rolagem

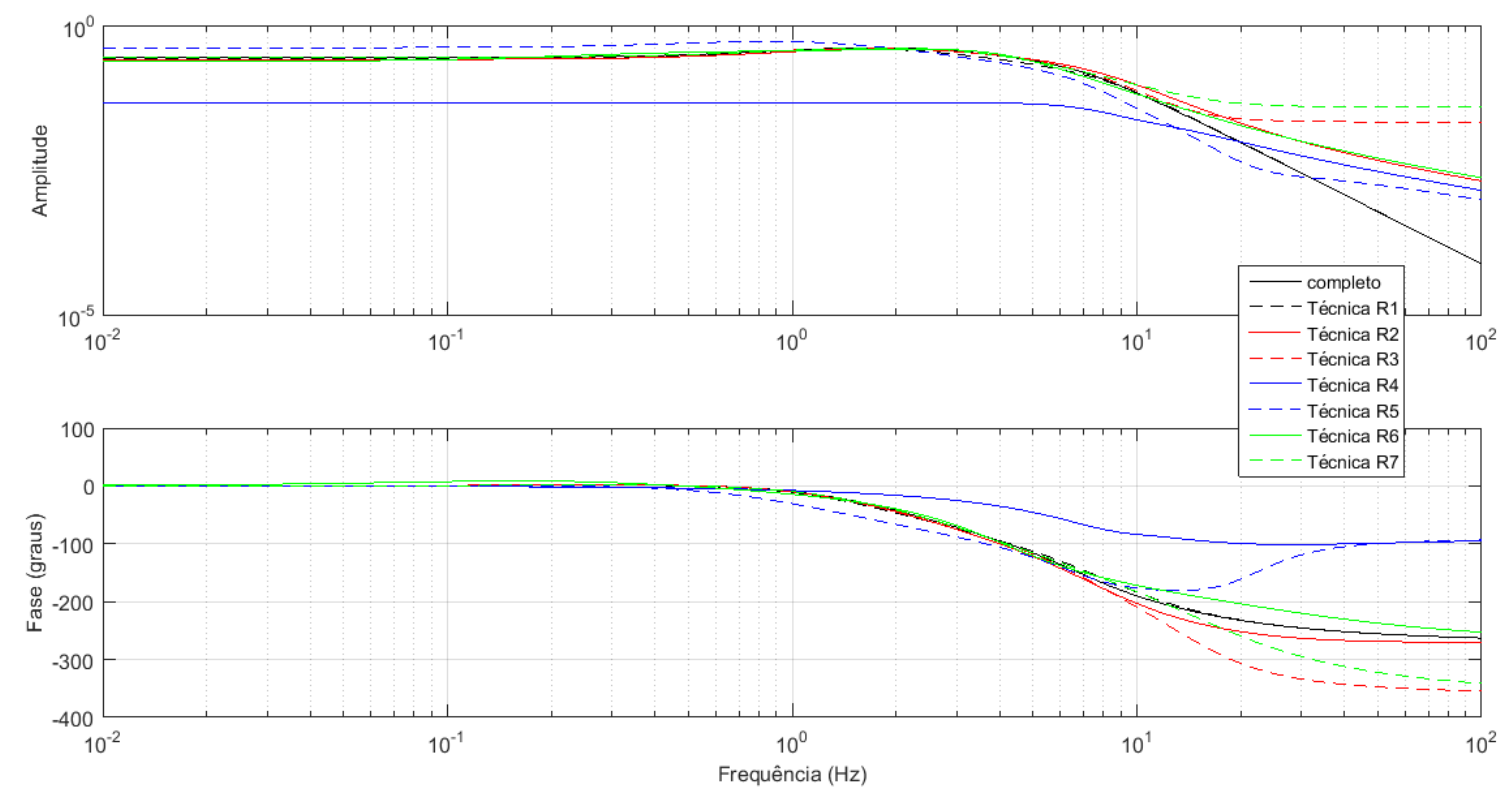

Também na figura 39, observa-se que os modelos MR2 e MR6 proporcionaram melhores aproximações do ângulo de fase do modelo completo, em relação aos outros modelos reduzidos.

A figura 40 mostra o diagrama de Bode do erro do ângulo de rolagem do veículo e a figura 
41 mostra o máximo valor singular da função de tranferência $G_{e}(s)$ do erro para cada modelo reduzido, calculadas pela equação 184 .

Figura 40 - Diagrama de bode do erro do ângulo de rolagem
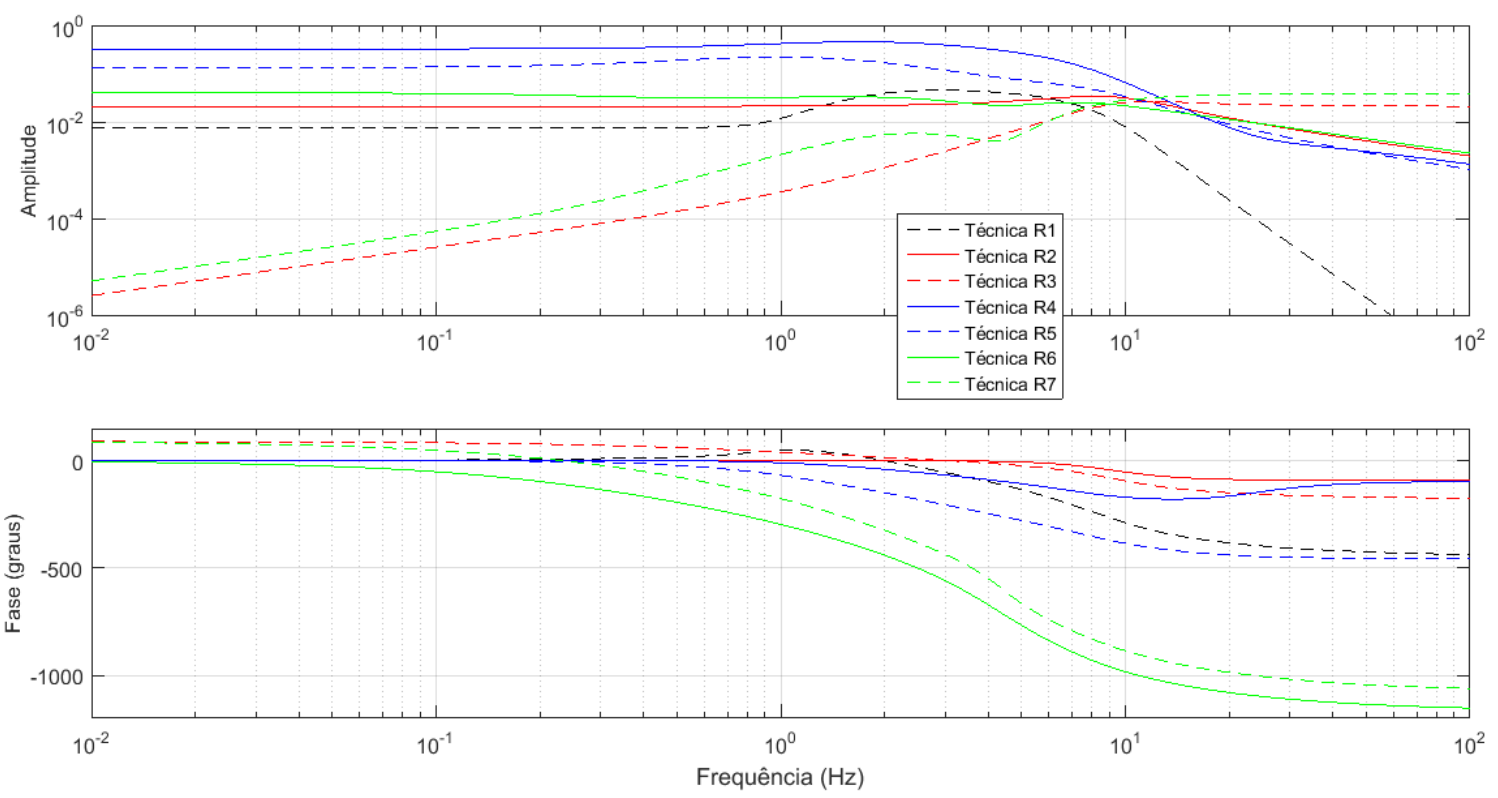

Figura 41 - Máximo valor singular da função de transferência do erro $\left(G_{e}(s)\right)$

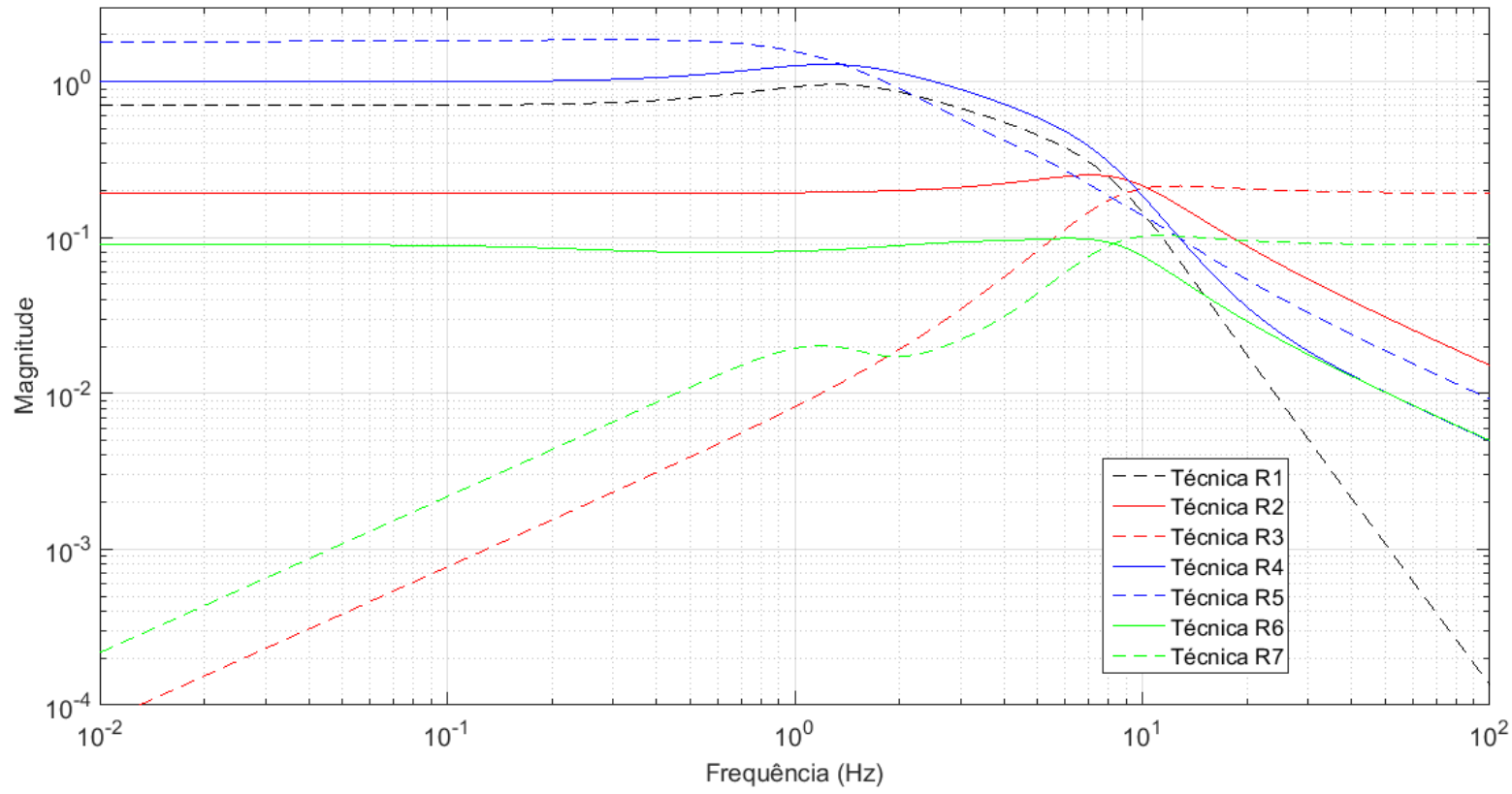

Observando as figuras 40 e 41 pode-se confirmar os seguintes comportamentos previstos no capítulo 11 .

- O erro dos modelos R3 e R7 é pequeno para frequências menores que $1 \mathrm{~Hz}$ e diminui conforme a frequência também diminui. Este comportamento ocorre pois a função de 
transferência do erro é nula em $s=0$ e consequentemente tem valores pequenos em frequências próximas a zero, conforme a equação 145 .

- O erro dos modelos reduzidos pelas técnicas R3 e R7 é mais elevado em frequências acima de $30 \mathrm{~Hz}$. Este comportamento ocorre pois as matrizes $D_{r}$ dos modelos reduzidos são diferentes da matriz $D$ do modelo completo, conforme a equação 144 . Portanto ao utilizar a equação 22, encontramos $G_{e}(\infty)=D-D_{r} \neq 0$.

- O erro dos modelos reduzidos pelas técnicas R1, R2, R5 e R6 apresenta pequena amplitude em frequências acima de $30 \mathrm{~Hz}$ e continua diminuindo com o aumento da frequência. Este comportamento ocorre pois as matrizes $D_{r}$ dos modelos reduzidos são iguais à matriz $D$ do modelo completo. Portanto ao utilizar a equação 22 encontramos $G_{e}(\infty)=$ $D-D_{r}=0$.

\subsubsection{Resultados por métricas de representatividade}

As figuras 42 a 44 mostram as métricas de representatividade $\mathcal{M}_{\mathcal{L}_{2}}, \mathcal{M}_{s s}$ e $\mathcal{M}_{s}$, calculadas a partir dos resultados para entrada degrau.

Vale lembrar que quanto menor o valor de uma métrica de representatividade, melhor é a aproximação que o modelo reduzido faz de uma determinada característica da resposta do modelo completo. Caso o valor de uma métrica seja nulo, o modelo reduzido reproduz com exatidão aquela característica da resposta que corresponde à norma em questão.

Figura 42 - Métrica de representatividade $\mathcal{M}_{\mathcal{L}_{2}}$ dos modelos reduzidos

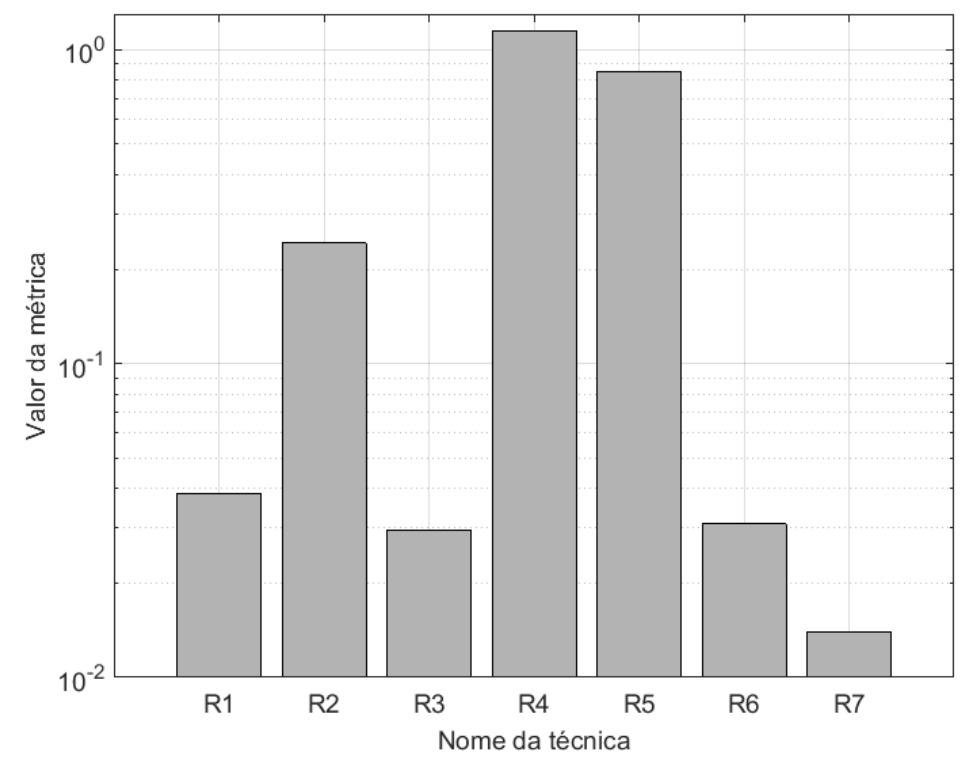

Como alguns modelos apresentam erro em estado estável para entrada degrau, o valor da 
norma $\mathcal{L}_{2}$ do erro no intervalo ] $-\infty, \infty$ [ tenderia a infinito para estes casos. Portanto ela foi calculada no intervalo entre $t_{0}=0$ s e $t_{f}=0,6$ s.

A figura 42 mostra a métrica $\mathcal{M}_{\mathcal{L}_{2}}$ de cada modelo reduzido. Esta métrica quantifica a capacidade que o modelo reduzido tem de reproduzir a resposta $y(t)$ do modelo completo no intervalo $t \in\left[t_{0}, t_{f}\right]$.

Observa-se na figura 42 que a métrica $\mathcal{M}_{\mathcal{L}_{2}}$ está abaixo de $3 \%$ para os modelos MR1, MR3, MR6 e MR7.

A figura 43 mostra a métrica $\mathcal{M}_{s s}$ de cada modelo reduzido, que quantifica a capacidade que o modelo reduzido tem de reproduzir os sobre-sinais do modelo completo. Pode-se ver nesta figura que os os valores da métrica $\mathcal{M}_{s s}$ é pequeno para os modelos MR3 e MR7. Isso significa que os sobresinais destes modelos são próximos dos sobresinais do modelo completo.

Figura 43 - Métrica de representatividade $\mathcal{M}_{s s}$ dos modelos reduzidos

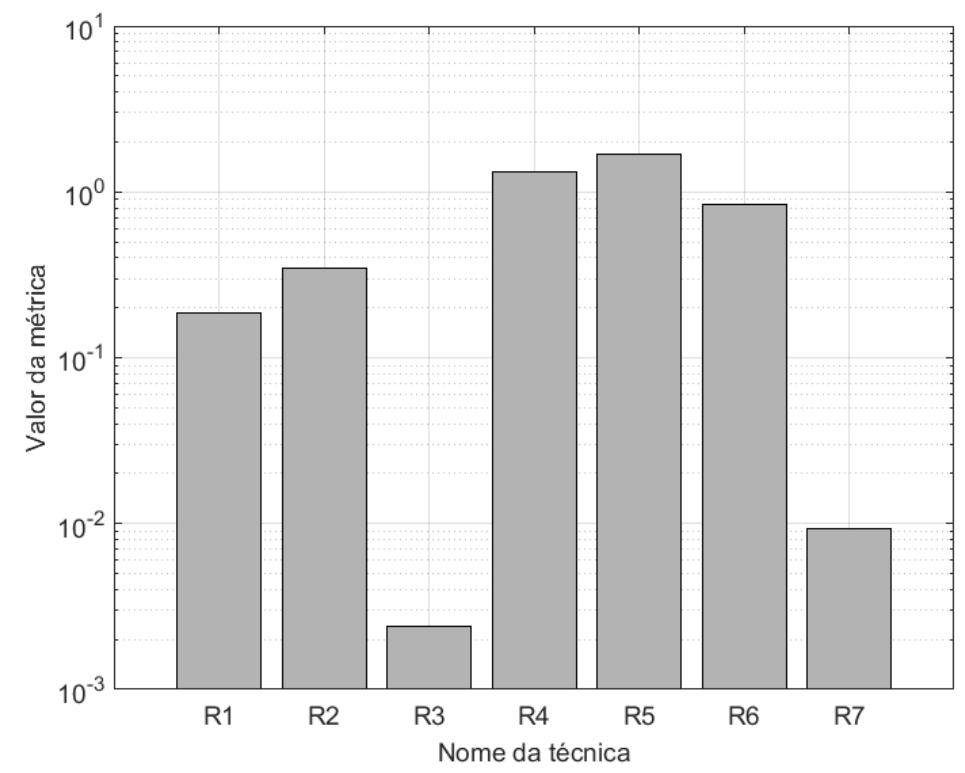

A figura 44 mostra a métrica $\mathcal{M}_{s}$ de cada modelo reduzido, que quantifica a capacidade que o modelo reduzido tem de reproduzir os tempos de acomodação do modelo completo. Observase nesta figura que a métrica $\mathcal{M}_{s}$ é pequena para os modelos MR1, MR2 e MR3. Isso mostra que os tempos de acomodação destes modelos são próximos dos tempos de acomodação do modelo completo.

Os valores de $\mathcal{M}_{s}$ para os modelos MR2 e MR3, que utilizam a BFM são iguais a 0,033 , enquanto para os modelos MR6 e MR7 que utilizam a base balanceada são respectivamente 3,5 e 0,35 . Isso mostra que os modelos reduzidos utilizando a BFM realizaram melhores aproximações para o tempo de acomodação do modelo completo em relação aos modelos que utilizaram a base balanceada. 
Figura 44 - Métrica de representatividade $\mathcal{M}_{s}$ dos modelos reduzidos

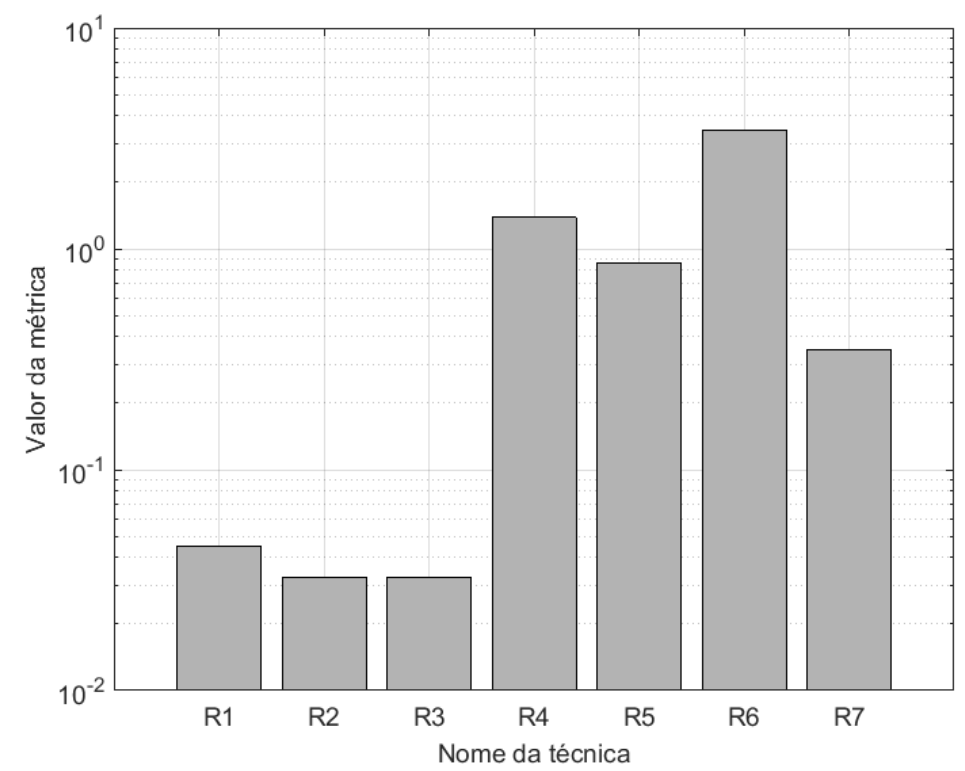

As figuras 45 e 46 mostram as métricas de representatividade $\mathcal{M}_{\mathcal{H}_{\infty}}$ e $\mathcal{M}_{\mathcal{H}_{2}}$ calculadas a partir dos resultados no domínio da frequência.

Figura 45 - Métrica de representatividade $\mathcal{M}_{\mathcal{H}_{\infty}}$ dos modelos reduzidos

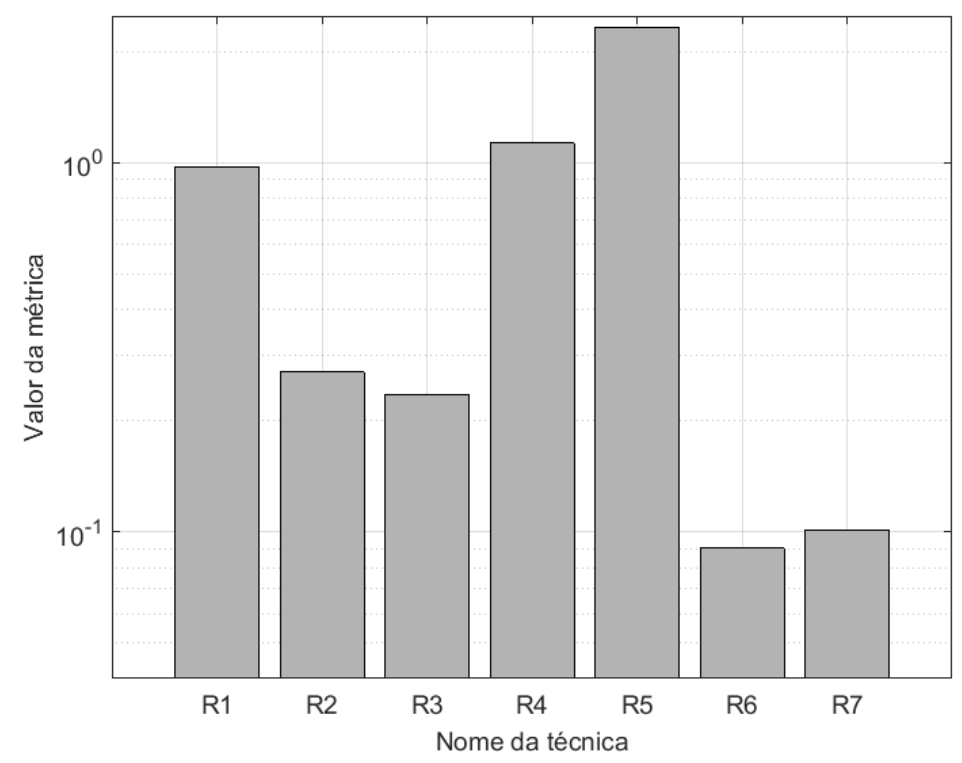

O valor da norma $\left\|G_{e}\right\|_{\mathcal{H}_{\infty}}$ para o modelo MR2 é 0,2025 e a aproximação deste valor calculada pela equação 231 é igual a 0,1855 . Portanto a estimativa da norma $\mathcal{H}_{\infty}$ foi calculada com erro de $8,4 \%$ antes que fossem eliminados os GDL do modelo completo.

O valor das normas $\left\|G_{e}\right\|_{\mathcal{H}_{\infty}}$ dos modelos MR6 e MR7 são respectivamente 0,0674 e 0,0755 e o limite superior calculado com a equação 232 é 0,245 . 
Observando a figura 45, pode-se perceber que os modelos MR6 e MR7, que utilizam a base balanceada, aproximam a norma $\mathcal{H}_{\infty}$ do sistema completo com erro inferior a $10 \%$. Os modelos MR2 e MR3, que utilizam a BFM, aproximam a norma $\mathcal{H}_{\infty}$ do sistema completo com erro inferior a $30 \%$.

Os resultados discutidos no parágrafo acima são coerentes com o limite máximo estabelecido no item 12.3 .1 para a norma $\mathcal{H}_{\infty}$ do modelo completo, que é de $33 \%$, válido para os modelos MR2, MR3, MR6 e MR7.

Figura 46 - Métrica de representatividade $\mathcal{M}_{\mathcal{H}_{2}}$ dos modelos reduzidos

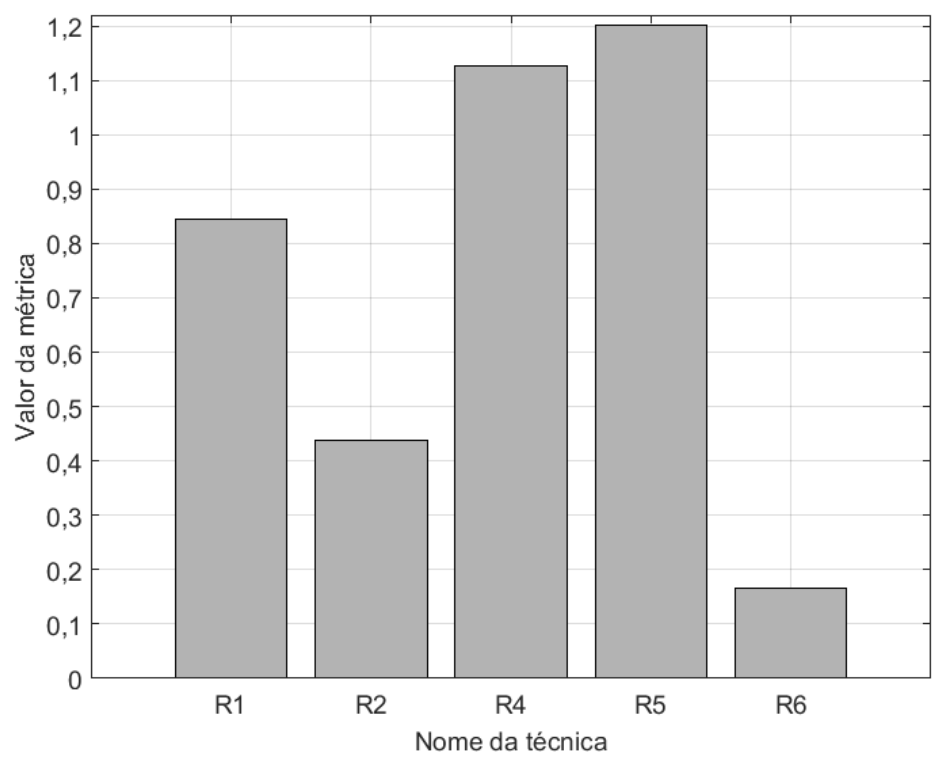

Os resultados das métricas $\mathcal{M}_{\mathcal{H}_{2}}$ dos modelos reduzidos MR3 e MR7 foram omitidos da figura 46 pois suas respectivas normas $\left\|G_{e}(s)\right\|_{\mathcal{H}_{2}}$ possuem valores que tendem a infinito, segundo a equação 174 . Isso ocorre pois suas respectivas matrizes $D_{e}=D-D_{r}$ não são nulas.

Observa-se na figura 46 que a métrica $\mathcal{M}_{\mathcal{H}_{2}}$ é pequena para o modelo MR6. Isso mostra que este modelo aproxima a função de transferência do modelo completo (em todo o espectro de frequência) melhor que os demais modelos analisados.

A figura 47 mostra a métrica $\mathcal{M}_{\text {eqmax }}$, que quantifica a capacidade do modelo reduzido para reproduzir uma resposta estática do modelo completo. Os resultados mostram que os modelos MR3 e MR7 possuem erro estático nulo para qualquer entrada, como foi previsto no capítulo 11 .

A figura 48 mostra a métrica $\sigma_{\max }\left(G_{e}(\infty)\right)$, que quantifica a capacidade do modelo reduzido para reproduzir o valor da função de transferência do modelo completo na situação $\lim _{\omega \rightarrow \infty} G(i \omega)$.

Os resultados mostram que os modelos MR1, MR2, MR4, MR5 e MR6 possuem valor nulo para esta métrica. Isso significa que a função de transferência dos modelos reduzidos é idêntica 
Figura 47 - Métrica de representatividade $\mathcal{M}_{\text {eqmax }}$ dos modelos reduzidos

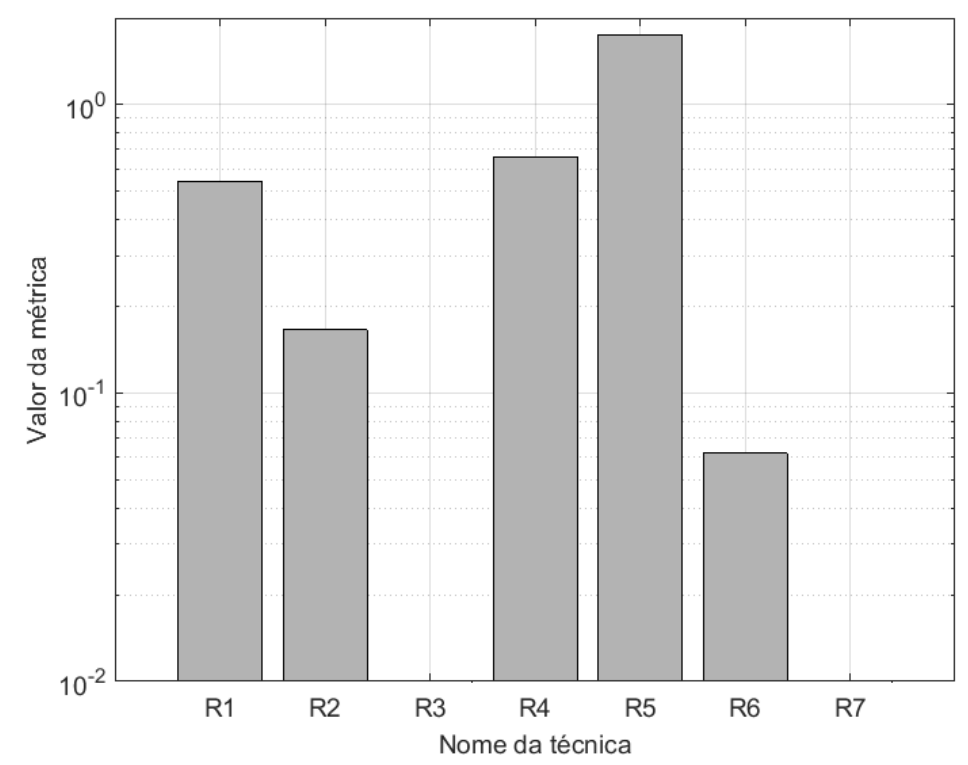

Figura 48 - Métrica de representatividade $\sigma_{\max }\left(G_{e}(\infty)\right)$ dos modelos reduzidos

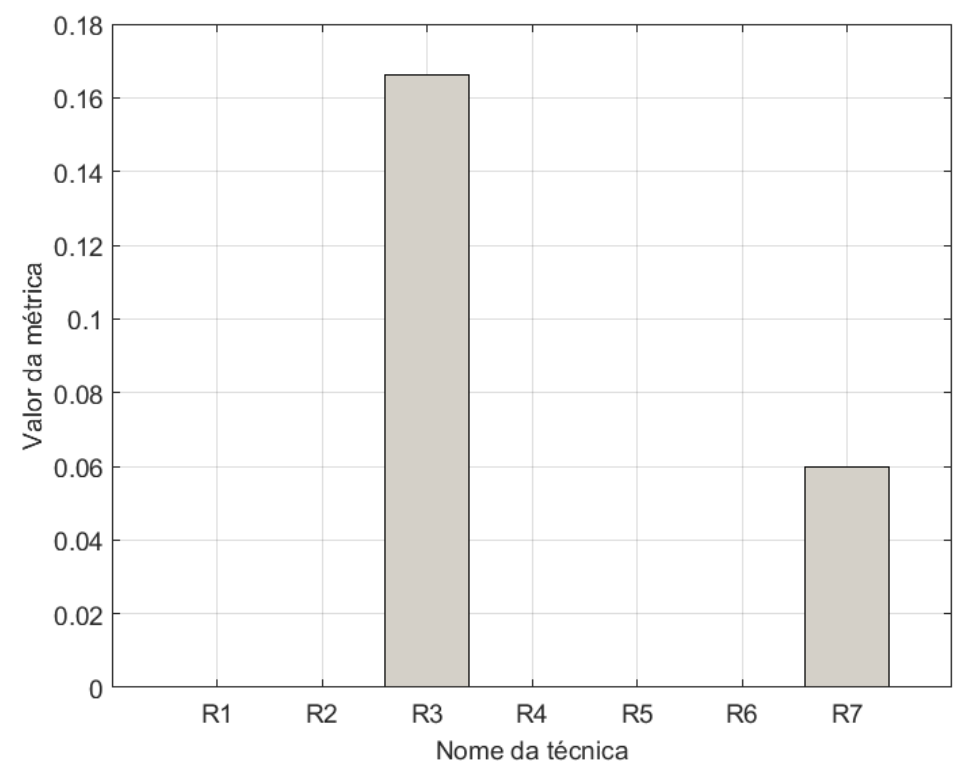

à do modelo completo na situação $\lim _{\omega \rightarrow \infty} G(i \omega)$, como foi previsto no capítulo 11 .

\subsubsection{Cálculo da representatividade total dos modelos reduzidos}

Após a apresentação dos resultados pelas métricas descritas no capítulo 11 será calculado o desempenho de cada modelo reduzido, que é determinado pela representatividade total $\mathcal{R}$ dos mesmos. A representatividade total será usada como critério de comparação entre os modelos reduzidos. 
De acordo com o método para cálculo da representatividade total de um modelo reduzido, descrito no item 9.5, é necessário escolher as métricas que quantificam as características do modelo completo que devem ser reproduzidas de modo mais fidedigno pelo modelo reduzido.

As características escolhidas pelo autor são:

- Capacidade de aproximar a resposta $y(t)$ do modelo completo no intervalo de tempo $\left[t_{0}, t_{f}\right]$, com $t_{0}=0 s$ e $t_{f}=0,6 s$.

- Capacidade de aproximar os sobre-sinais do modelo completo.

- Capacidade de aproximar os tempos de acomodação do modelo completo.

- Capacidade de aproximar a norma $\mathcal{H}_{\infty}$ da função de transferência do modelo completo.

Desta maneira, o vetor de métricas $\mathcal{M}$, que será utilizado na equação 191 é:

$$
\mathcal{M}=\left[\mathcal{M}_{\mathcal{L}_{2}} ; \mathcal{M}_{s s} ; \mathcal{M}_{s} ; \mathcal{M}_{\mathcal{H}}\right]^{T}
$$

A matriz de ponderação $P$ definida pelo analista, de acordo com as características que ele deseja priorizar, é $P=\operatorname{diag}(2 ; 2 ; 1 ; 2)$. Isso significa que a terceira métrica do vetor $\mathcal{M}$ tem menor ponderação que as outras na quantificação do desempenho do modelo reduzido.

Tabela 15 - Representatividade dos modelos reduzidos

\begin{tabular}{|c|c|}
\hline $\begin{array}{c}\text { Modelo de } \\
\text { reduzido }\end{array}$ & $\begin{array}{c}\text { Representatividade } \\
\mathcal{R}\end{array}$ \\
\hline MR1 & 0,72 \\
\hline MR2 & 1,40 \\
\hline MR3 & 2,97 \\
\hline MR4 & 0,31 \\
\hline MR5 & 0,24 \\
\hline MR6 & 0,27 \\
\hline MR7 & 2,65 \\
\hline
\end{tabular}

O valor da representatividade $\mathcal{R}$ para cada modelo foi calculado segundo a equação 191 e os valores são mostrados na tabela 15

Vale lembrar que quanto maior o valor de $\mathcal{R}$, maior a capacidade do modelo reduzido de reproduzir as características do modelo completo (elencadas no vetor $\mathcal{M}$ ).

Pode-se concluir a partir dos dados da tabela 15, que o modelo MR3 é o que melhor reproduz as características determinadas acima. 
12.4 INFLUÊNCIA DO CRITERIO DE SELEÇÃO NA RESPOSTA DO MODELO REDUZIDO UTILIZANDO O MODELO DE VEÍCULO DE PASSEIO

Neste item, será analisada a inflência do critério de seleção nas métricas de representatividade do modelo reduzido. Serão comparados os resultados de modelos reduzidos obtidos através de diferentes critérios de seleção na BFM.

Para tal análise, foram analisadas 4 técnicas de redução com as seguintes características em comum:

- Projeção na base de formas modais (BFM).

- Eliminação de GDL por truncamento direto.

- Nenhum método de correção implementado.

A tabela 16 mostra os modos de vibrar organizados segundo os critérios de seleção para BFM apresentados nos itens 12.2.1 a 12.2.4. Os modos estão organizados de maneira que o mais dominante encontra-se no início da lista e o menos dominante no final.

Tabela 16 - Comparação da ordem de seleção dos modos de vibrar segundo diferentes critérios

\begin{tabular}{|c|c|}
\hline $\begin{array}{c}\text { Critério } \\
\text { de seleção }\end{array}$ & $\begin{array}{c}\text { Ordem de importância } \\
\text { dos modos }\end{array}$ \\
\hline CSF & $1,2,3,4,5,6,7$ \\
\hline CSFA & $1,2,3,6,7,4,5$ \\
\hline CSCO & $3,1,2,4,5,6,7$ \\
\hline CSFT & $4,3,1,5,2,6,7$ \\
\hline
\end{tabular}

Baseado na tabela 16 observa-se que os critérios de seleção na BFM aqui apresentados podem indicar uma ordem de candidatos a eliminação diferente entre si.

Para que exista uma apresentação mais objetiva dos resultados, o autor separou as métricas de representatividade em 3 grupos.

- Primeiro grupo: foram consideradas as métricas que quantificam as características da resposta transiente de um modelo reduzido: $\mathcal{M}_{\mathcal{L}_{2}}, \mathcal{M}_{s s}$ e $\mathcal{M}_{s}$. Para o primeiro grupo, foi criada a métrica $\mathcal{M}_{t r}$, que quantifica a capacidade do modelo reduzido em reproduzir a resposta transiente do modelo completo.

$$
\mathcal{M}_{t r}=\left(\left[\mathcal{M}_{\mathcal{L}_{2}} ; \mathcal{M}_{s s} ; \mathcal{M}_{s}\right]^{T}\left[\mathcal{M}_{\mathcal{L}_{2}} ; \mathcal{M}_{s s} ; \mathcal{M}_{s}\right]\right)^{\frac{1}{2}}
$$


- Segundo grupo: foram consideradas as métricas que quantificam as características da resposta no domínio da frequência de um modelo reduzido: $\mathcal{M}_{\mathcal{H}_{2}}, \mathcal{M}_{\mathcal{H}_{\infty}}$. Para o segundo grupo, foi criada a métrica $\mathcal{M}_{r f}$, que quantifica a capacidade do modelo reduzido em reproduzir a resposta da função de transferência do modelo completo.

$$
\mathcal{M}_{f t}=\left(\left[\mathcal{M}_{\mathcal{H}_{2}} ; \mathcal{M}_{\mathcal{H}_{\infty}}\right]^{T}\left[\mathcal{M}_{\mathcal{H}_{2}} ; \mathcal{M}_{\mathcal{H}_{\infty}}\right]\right)^{\frac{1}{2}}
$$

- Terceiro grupo: foram consideradas as métricas que quantificam as características da resposta estática de um modelo reduzido: $\mathcal{M}_{\text {eqmax }}$. Como o terceiro grupo possui apenas uma métrica, esta será utilizada para a apresentação dos resultados.

Para cálculo dos resultados, a métrica $\mathcal{M}_{\mathcal{L}_{2}}$ foi calculada no intervalo onde $t_{0}=0 \mathrm{e}$ $t_{f}=0,6$.

Observa-se nas figuras 49 e 50 que o modelo que utiliza o critério de seleção CSFA tem maiores valores das métricas $\mathcal{M}_{t r}$ e $\mathcal{M}_{f r}$ que os demais quando são reduzidos 2, 4 e $6 \mathrm{GDL}$. Isso significa que os outros modelos tem melhor capacidade de aproximar tanto as características transientes quanto a resposta da função de transferência do modelo completo quando são reduzidos de 2 a $6 \mathrm{GDL}$.

Figura 49 - Métrica de representatividade $\mathcal{M}_{t r}$ dos modelos reduzidos

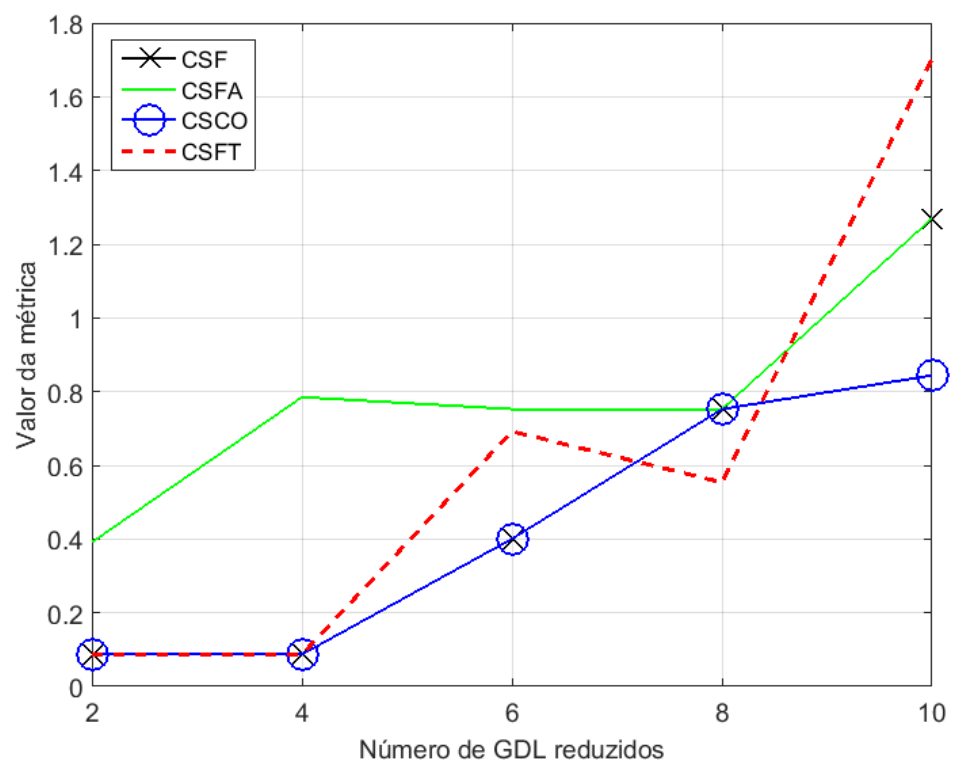

Também pode-se ver que as técnicas que utilizam os critérios de seleção CSF, CSFA e CSCO têm respostas idênticas quando são eliminados $8 \mathrm{GDL}$. Isso ocorre pois os 4 modos menos dominantes (modos 4, 5, 6 e 7) dos três critérios são iguais. Observa-se que mesmo que a 
Figura 50 - Métrica de representatividade $\mathcal{M}_{f r}$ dos modelos reduzidos

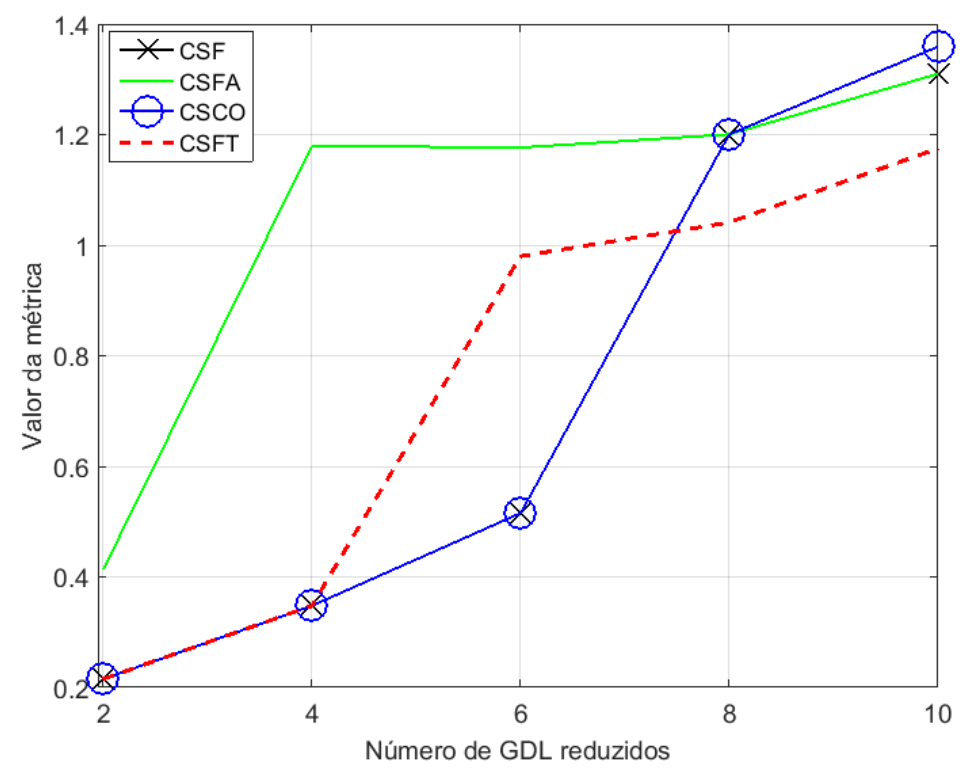

Figura 51 - Métrica de representatividade $\mathcal{M}_{\text {eqmax }}$ dos modelos reduzidos

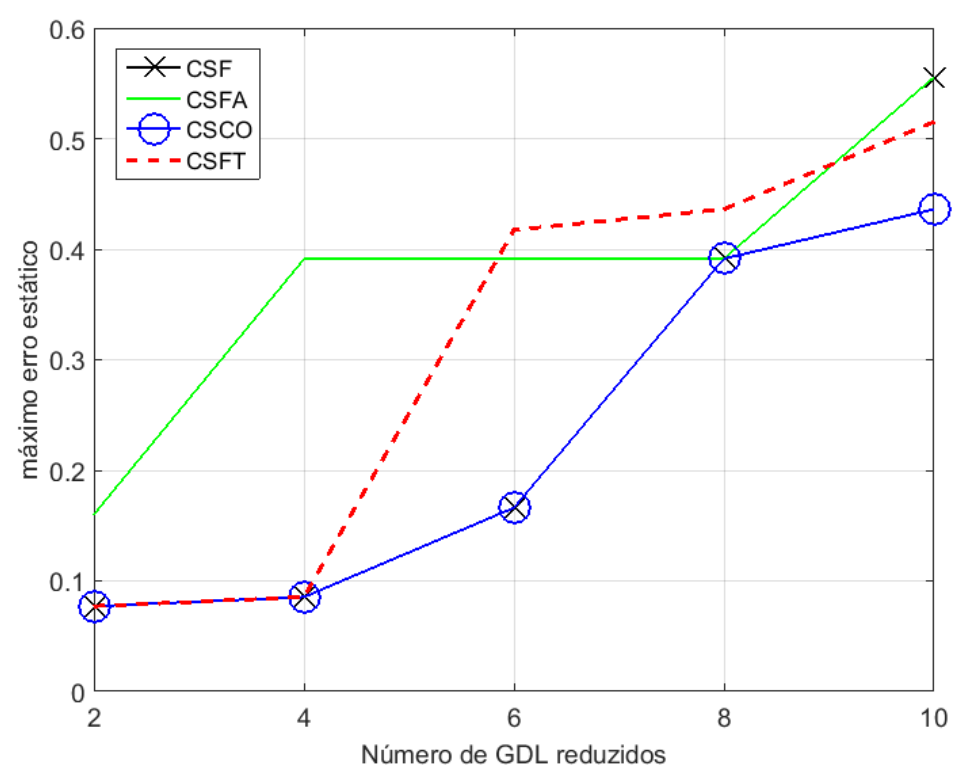

ordem em que estes modos são organizados seja diferente, os modelos obtidos pelas 3 técnicas em questão são idênticos quando são eliminados $8 \mathrm{GDL}$.

Portanto independente da ordem em que cada critério prioriza os GDL, quando as listas de GDL a serem eliminados são iguais, os modelos reduzidos serão idênticos desde que a base de projeção, o método de eliminação e o método de correção sejam iguais em ambos os casos. 
12.5 INFLUÊNCIA DO NÚMERO DE GDL REDUZIDOS UTILIZANDO O MODELO DE TREM

No item 12.5. será mostrada a influência que o número de GDL eliminados tem na resposta dos modelos reduzidos pelas técnicas apresentadas no capítulo 11. Para isto, foi utilizado o modelo de trem descrito no item 10.2 .

Os autovalores do modelo completo de trem estão indicados na figura 52 . Pode-se observar que modelo completo de trem é assintoticamente estável isto é, possui autovalores com parte real menor que zero.

Figura 52 - Autovalores do modelo completo de trem

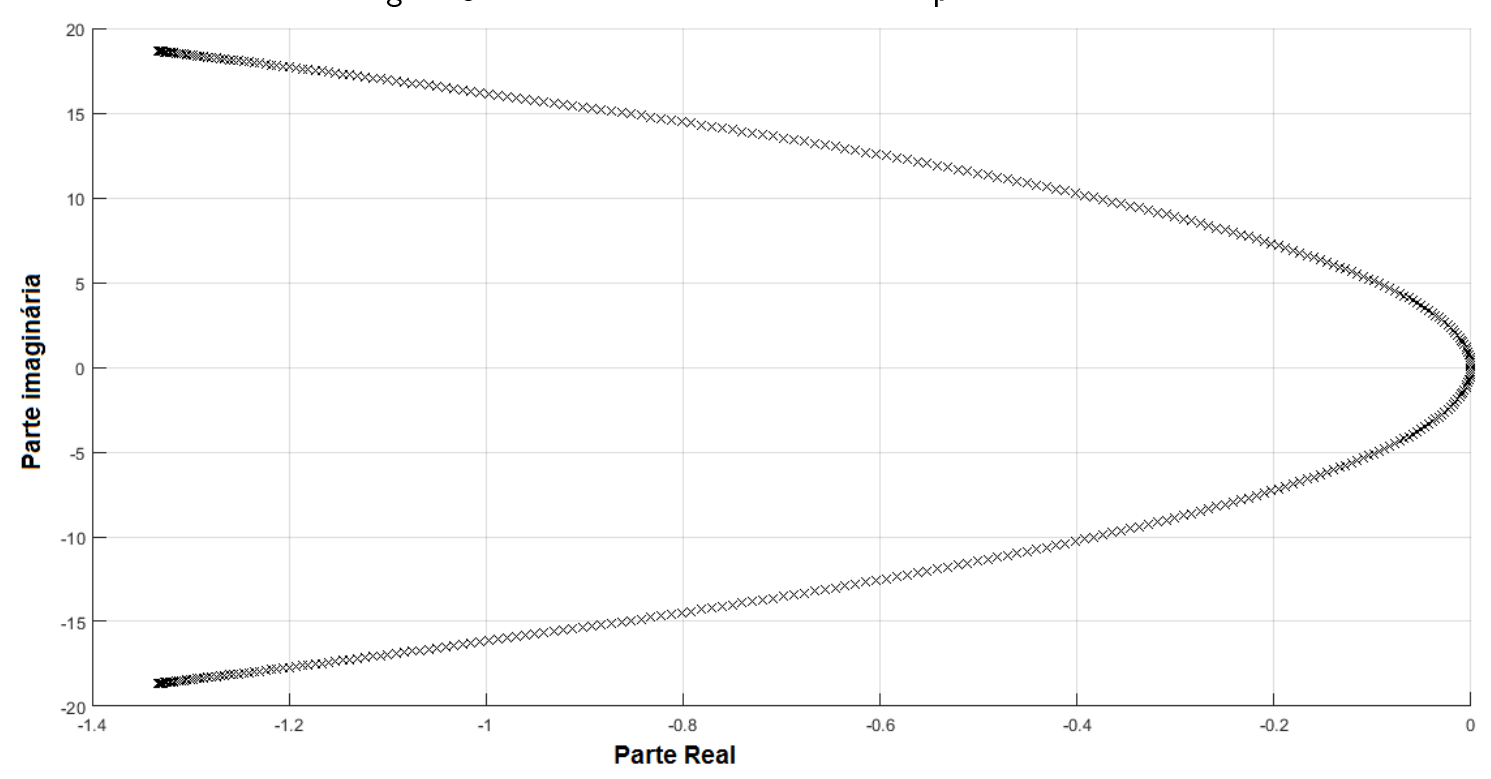

O modelo completo de trem possui $398 \mathrm{GDL}$. Para cada técnica de redução da tabela 5 foram gerados 36 modelos reduzidos iniciando com 20 e chegando a $380 \mathrm{GDL}$ eliminados, em intervalos de $10 \mathrm{GDL}$.

Os resultados da técnica de redução R1 mostrada na tabela 5 não serão mostrados nesta análise pois a implementação do critério de seleção baseado no conhecimento do analista se torna inviável quando o número de GDL no sistema é elevado.

Conforme orientações descritas no item 11.5 como o modelo completo de trem tem uma entrada, ao aplicar a técnica $\mathrm{R} 5$, o vetor inicial para a base BKMA é igual à matriz $B$ do modelo completo. (ANTOULAS; SORENSEN; GUGERCIN, 2001)

Os resultados da técnica R5 não serão mostrados nesta análise pois não existem modelos reduzidos que sejam estáveis ou bem condicionados no intervalo de valores dos GDL que foram eliminados do sistema. Tal verificação foi realizada iniciando com 20 e chegando a $380 \mathrm{GDL}$ eliminados, em intervalos de $1 \mathrm{GDL}$. 


\subsubsection{Resultados por metricas de representatividade}

As figuras 53, 54, 55, 56 e 57 mostram respectivamente o valor das métricas $\mathcal{M}_{\mathcal{L}_{2}}, \mathcal{M}_{\mathcal{H}_{2}}$, $\mathcal{M}_{\mathcal{H}_{\infty}}, \mathcal{M}_{\text {eqmax }}$ e $\mathcal{M}_{s s}$ (em escala logarítmica) em função do número de GDL eliminados.

A figura 53 mostra a métrica $\mathcal{M}_{\mathcal{L}_{2}}$ calculada segundo a equação 175 no intervalo $\left[t_{0}, t_{f}\right]$ com $t_{0}=0 \mathrm{~s}$ e $t_{f}=50 \mathrm{~s}$ utilizando entrada degrau de valor unitário. Esta métrica é igual à razão entre as normas $\mathcal{L}_{2}$ do erro e do modelo completo. Em outras palavras, esta métrica está relacionada ao erro da resposta $y(t)$ do modelo reduzido no intervalo de tempo $\left[t_{0}, t_{f}\right]$.

Figura 53 - Métrica de representatividade $\mathcal{M}_{\mathcal{L}_{2}}$ em função do número de GDL reduzidos

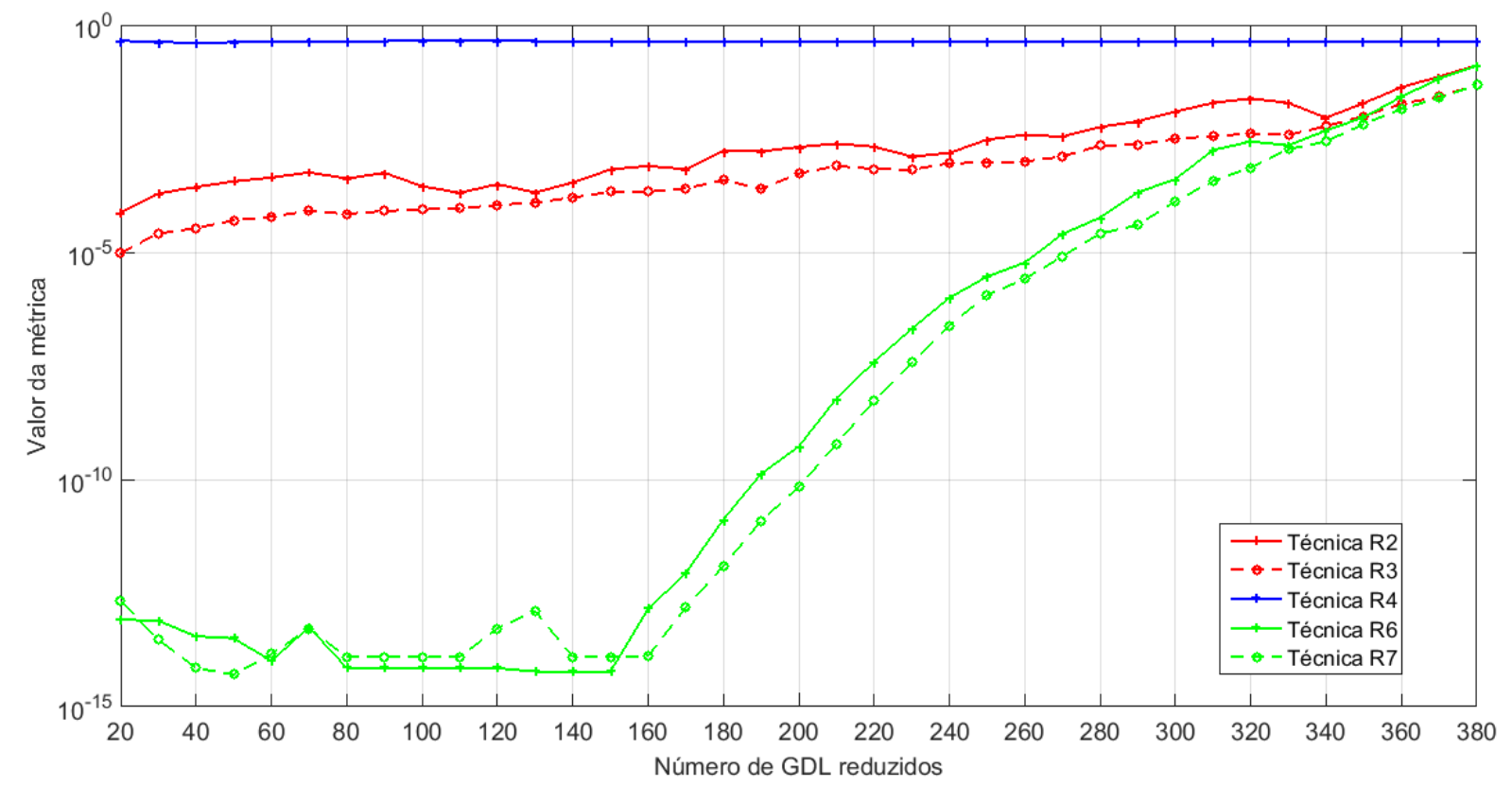

Vale lembrar que quanto menor o valor das métricas de representatividade, menor é o erro associado à característica que a métrica representa. Ou seja, maior será a capacidade que o modelo reduzido tem de reproduzir determinada característica do modelo completo.

A partir da figura 53 observa-se que a norma $\mathcal{L}_{2}$ do erro dos modelos reduzidos pelas técnicas R6 e R7 é menor que a dos demais modelos no intervalo entre 20 a 350 GDL eliminados. Isso significa que no intervalo citado, os modelos reduzidos pelas técnicas R6 e R7 reproduziram com maior fidelidade a resposta $y(t)$ do sistema completo (no intervalo de tempo $\left[t_{0}, t_{f}\right]$ ) quando submetido a uma entrada degrau.

Os modelos reduzidos pela técnica R4 apresentaram piores resultados para a métrica $\mathcal{M}_{\mathcal{L}_{2}}$, comparados com as demais técnicas analisadas, em todo o intervalo entre 20 e $380 \mathrm{GDL}$ eliminados.

Os valores das métricas $\mathcal{M}_{\mathcal{L}_{2}}$ e $\mathcal{M}_{\mathcal{H}_{2}}$ dos modelos que utilizam a base balanceada têm pouca variação no intervalo de 20 a $160 \mathrm{GDL}$ eliminados. Quando aumentamos o número de GDL 
eliminados acima de 160 , os valores das métricas em questão aumentam consideravelmente. Isto pode ser observado nas figuras 53 e 56

O comportamento descrito no parágrafo anterior revela que as normas $\mathcal{L}_{2}$ e $\mathcal{H}_{2}$ do erro dos modelos reduzidos na base balanceada passa a aumentar drasticamente quando são eliminados mais de $160 \mathrm{GDL}$. Isso nos faz concluir que existe um intervalo entre 20 e $160 \mathrm{GDL}$ onde o erro destes modelos reduzidos é menos comprometido com o aumento do número de GDL eliminados.

A partir da figura 53, observa-se que a métrica $\mathcal{M}_{\mathcal{L}_{2}}$ dos modelos reduzidos pelas técnicas R6 e R7 é menor que $10^{-5}$ no intervalo entre 20 a $260 \mathrm{GDL}$ eliminados. Isso significa que, utilizando a equação 175 no intervalo citado, os modelos reduzidos pelas técnicas R6 e R7 reproduziram a norma $\mathcal{L}_{2}$ do sistema completo com erro relativo inferior a $0.001 \%$. Analogamente no mesmo intervalo, os modelos reduzidos reproduziram a norma $\mathcal{L}_{2}$ do sistema completo com erro relativo inferior a $0.4 \%$ para a técnica $\mathrm{R} 2$ e $0.1 \%$ para a técnica R3.

Portanto pode-se concluir que dentre as técnicas analisadas, as técnicas R6 e R7 são as mais adequadas para reproduzir com maior fidelidade a resposta $y(t)$ do modelo reduzido no intervalo de tempo $[0,50]$ segundos.

A figura 54 mostra a métrica $\mathcal{M}_{\mathcal{H}_{2}}$ (calculada segundo a equação 179) em função do número de GDL reduzidos. Esta métrica é igual à razão entre as normas $\mathcal{H}_{2}$ do erro e do modelo completo. Em outras palavras, esta métrica está relacionada ao erro da função de transferência $G_{r}(s)$ do modelo reduzido no intervalo $s \in[0 ; \infty[$.

Figura 54 - Métrica de representatividade $\mathcal{M}_{\mathcal{H}_{2}}$ em função do número de GDL reduzidos

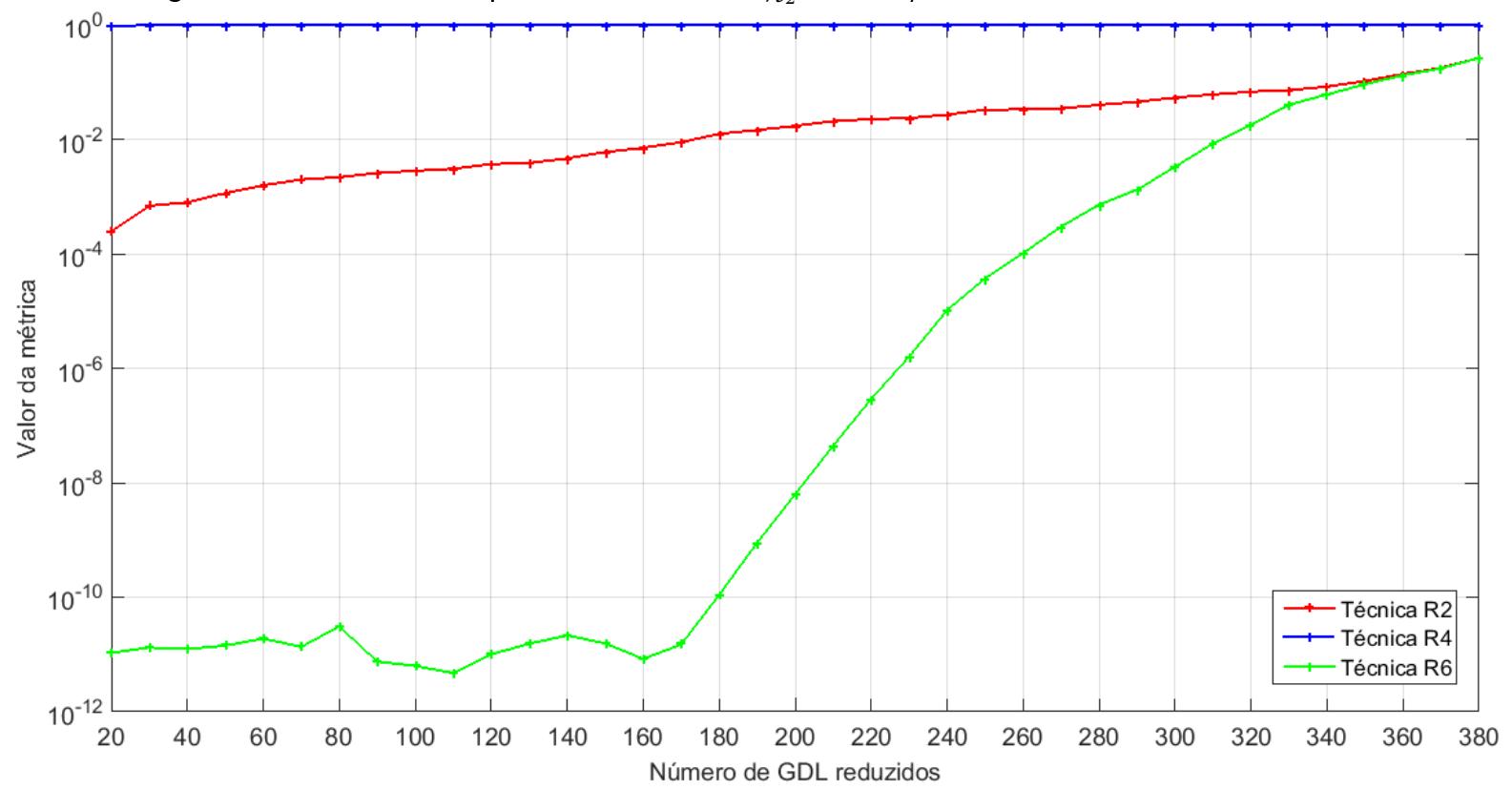

Os resultados das métricas $\mathcal{M}_{\mathcal{H}_{2}}$ dos modelos reduzidos pelas técnicas R3 e R7 foram 
omitidos da figura 54 pois suas respectivas normas $\left\|G_{e}(s)\right\|_{\mathcal{H}_{2}}$ possuem valores que tendem a infinito, segundo a equação 174 . Isso ocorre pois suas respectivas matrizes $D_{e}=D-D_{r}$ não são nulas.

A partir da figura 54, observa-se que a norma $\mathcal{H}_{2}$ do erro dos modelos reduzidos pela técnica R6 é menor que a dos demais modelos no intervalo entre 20 a 370 GDL eliminados. Isso significa que no intervalo citado, os modelos reduzidos pela técnica R6 reproduziram com maior fidelidade a resposta da função de transferência $G(s)$ do sistema completo no intervalo $s \in[0 ; \infty[$.

Os modelos reduzidos pela técnica R4 apresentaram piores resultados para a métrica $\mathcal{M}_{\mathcal{H}_{2}}$, comparados com as demais técnicas analisadas, em todo o intervalo entre 20 e $380 \mathrm{GDL}$ eliminados.

A partir da figura 54, observa-se que a métrica $\mathcal{M}_{\mathcal{H}_{2}}$ dos modelos reduzidos pela técnica R6 é menor que $10^{-4}$ no intervalo entre 20 a $260 \mathrm{GDL}$ eliminados. Isso significa que, utilizando a equação 179 no intervalo citado, os modelos reduzidos pela técnica R6 reproduziram a norma $\mathcal{H}_{2}$ do sistema completo com erro relativo inferior a $0.01 \%$. Analogamente no intervalo entre 20 e $170 \mathrm{GDL}$ eliminados, os modelos reduzidos pela técnica R2 reproduziram a norma $\mathcal{H}_{2}$ do sistema completo com erro relativo inferior a $1 \%$.

Portanto pode-se concluir que dentre as técnicas analisadas, a técnica R6 é a mais adequada para reproduzir com maior fidelidade a resposta função de transferência $G(s)$ do sistema completo no intervalo $s \in[0 ; \infty[$.

A figura 55 mostra a métrica $\mathcal{M}_{\mathcal{H}_{\infty}}$ (calculada segundo a equação 183) em função do número de GDL reduzidos. Esta métrica é igual à razão entre as normas $\mathcal{H}_{\infty}$ do erro e do modelo completo. Em outras palavras, esta métrica está associada à norma $\mathcal{H}_{\infty}$ do erro, que é o máximo maior valor singular da função de transferência do erro $G_{e}(s)$ no intervalo $s \in[0 ; \infty[$.

Os modelos reduzidos pela técnica R4 apresentaram piores resultados para a métrica $\mathcal{M}_{\mathcal{H}_{\infty}}$, comparados com as demais técnicas analisadas, em todo o intervalo entre 20 e $380 \mathrm{GDL}$ eliminados.

A partir da figura 55, observa-se que a métrica $\mathcal{M}_{\mathcal{H}_{\infty}}$ dos modelos reduzidos pelas técnicas R6 e R7 é menor que a dos demais modelos no intervalo entre 20 a $380 \mathrm{GDL}$ eliminados. Isso significa que no intervalo citado, os modelos reduzidos pelas técnicas R6 e R7 reproduziram com maior fidelidade a norma $\mathcal{H}_{\infty}$ do modelo completo.

A partir da figura 55, observa-se que a métrica $\mathcal{M}_{\mathcal{H}_{\infty}}$ dos modelos reduzidos pelas técnicas R6 e R7 é menor que $10^{-4}$ no intervalo entre 20 a $300 \mathrm{GDL}$ eliminados. Isso significa que, utilizando a equação 183 no intervalo citado, os modelos reduzidos pelas técnicas R6 e R7 reproduziram a norma $\mathcal{H}_{\infty}$ do sistema completo com erro relativo inferior a $0.01 \%$. Analoga- 
Figura 55 - Métrica de representatividade $\mathcal{M}_{\mathcal{H}_{\infty}}$ em função do número de GDL reduzidos

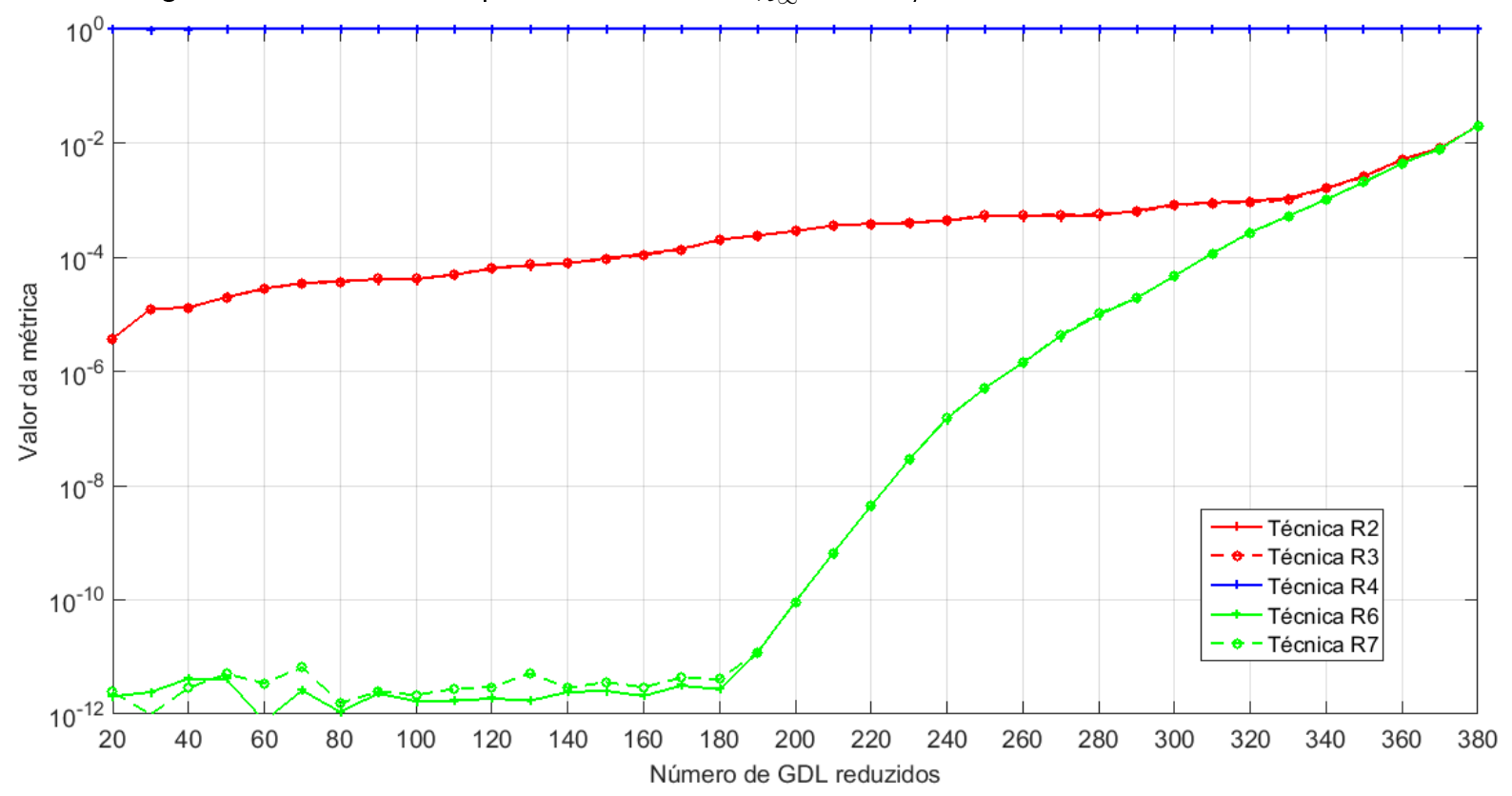

mente no intervalo entre 20 e $150 \mathrm{GDL}$ eliminados, os modelos reduzidos pelas técnicas R2 e R3 reproduziram a norma $\mathcal{H}_{\infty}$ do sistema completo com erro relativo inferior a $0.01 \%$.

A figura 56 mostra a métrica $\mathcal{M}_{\text {eqmax }}$ (calculada segundo a equação 190 em função do número de GDL reduzidos. Esta métrica é igual á máxima norma do erro estático considerando todas as entradas tipo degrau $u_{e q}$ possíveis. Em outras palavras, esta métrica está associada à capacidade que o modelo reduzido tem de reproduzir a resposta de equilíbrio $y_{e q}$ do modelo completo para uma entrada degrau.

Figura 56 - Métrica de representatividade dos modelos $\mathcal{M}_{\text {eqmax }}$ em função do número de GDL reduzidos

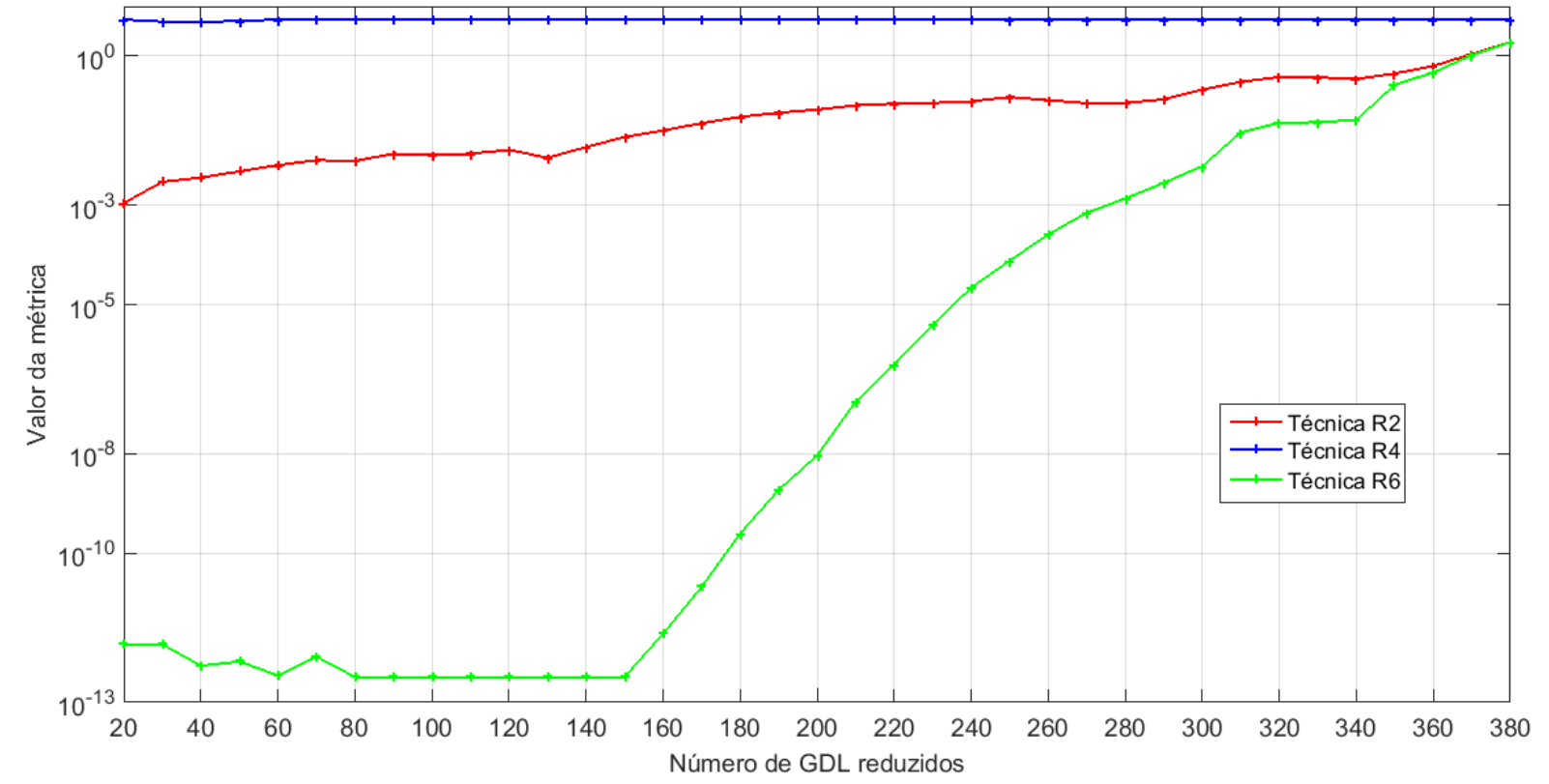


De acordo com as discussões apresentadas no capítulo 11. os valores dos erros estáticos dos modelos reduzidos pelas técnicas R3 e R7 devem ser nulos. Consequentemente os valores da métrica $\mathcal{M}_{\text {eqmax }}$ também devem ser nulos para estes modelos. Em outras palavras, os modelos reduzidos pelas técnicas R3 e R7 reproduzem com exatidão as respostas estáticas $y_{e q}$ do modelo completo para qualquer entrada que seja aplicada.

Como a figura 56 mostra os valores da métrica $\mathcal{M}_{\text {eqmax }}$ em escala logarítmica, os valores referentes aos modelos reduzidos pelas técnicas R3 e R7 não foram mostrados no gráfico.

A partir da figura 54 observa-se que os valores da métrica $\mathcal{M}_{\text {eqmax }}$ dos modelos reduzidos pela técnica R6 são menores que a dos modelos reduzidos pela técnica R2 (no intervalo entre 20 a $380 \mathrm{GDL}$ eliminados). Isso significa que no intervalo citado, a técnica R6 apresenta vantagem sobre a técnica R2 para reproduzir com fidelidade a resposta de equilíbrio $y_{e q}$ do modelo completo para uma entrada degrau.

Os modelos reduzidos pela técnica R4 apresentaram piores resultados para a métrica $\mathcal{M}_{\text {eqmax }}$, comparados com as demais técnicas analisadas, em todo o intervalo entre 20 e 380 GDL eliminados.

Como os modelos reduzidos pelas técnicas R3 e R7 possuem valor nulo para a métrica $\mathcal{M}_{\text {eqmax }}$, estas técnicas são as mais adequadas para reproduzir com fidelidade a resposta de equilíbrio $y_{e q}$ do modelo completo para uma entrada degrau

A figura 57 mostra a métrica $\mathcal{M}_{s s}$ calculada segundo a equação 172 Esta métrica está relacionada com os erros dos sobre-sinais do modelo reduzido em relação ao sistema completo quando submetidos a uma entrada degrau.

Figura 57 - Métrica de representatividade dos modelos $\mathcal{M}_{s s}$ em função do número de GDL reduzidos

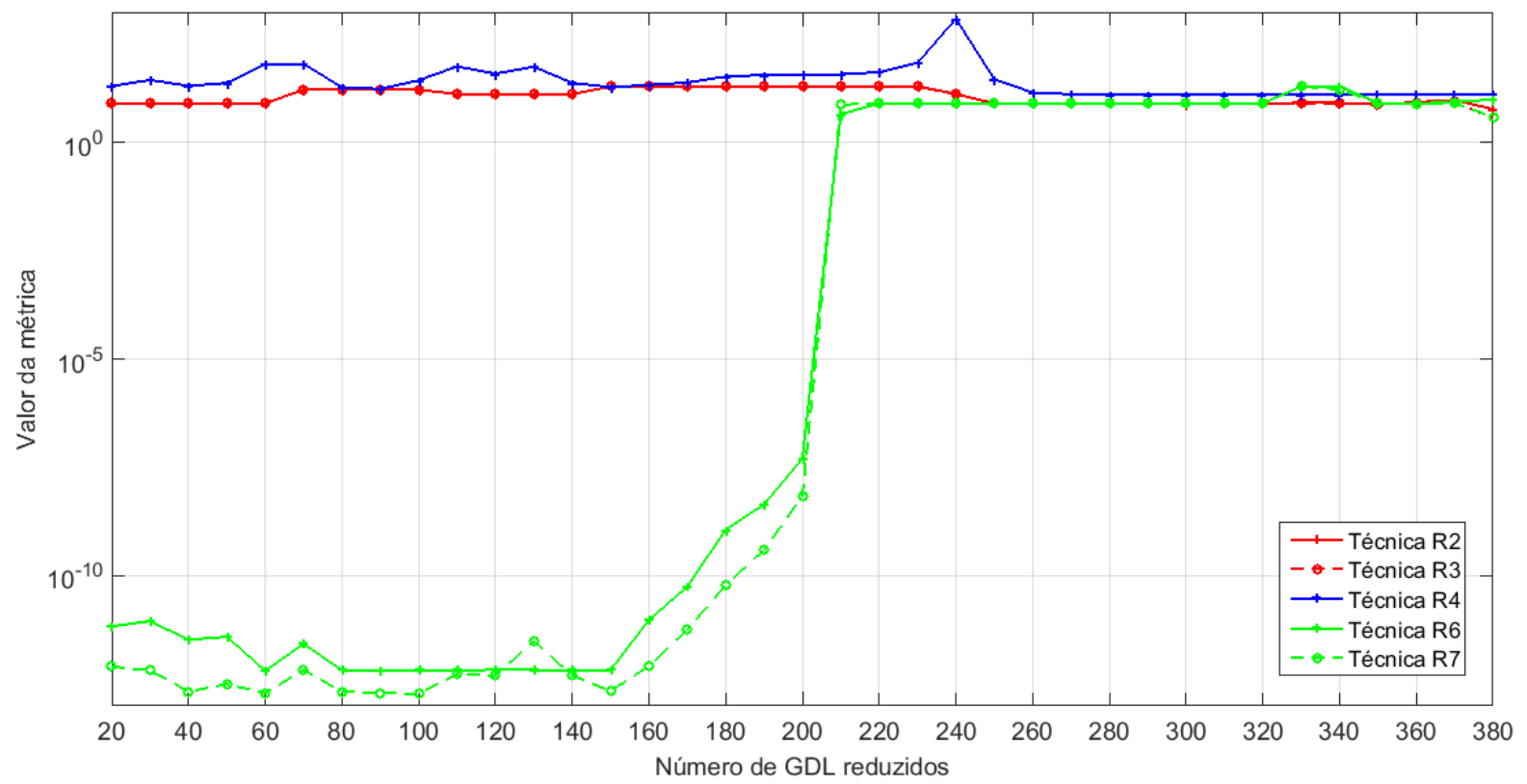


A partir da figura 57, observa-se que a métrica $\mathcal{M}_{s s}$ dos modelos reduzidos pelas técnicas R6 e R7 é menor que a dos demais modelos no intervalo entre 20 a $260 \mathrm{GDL}$ eliminados. Isso significa que no intervalo citado, os modelos reduzidos pelas técnicas R6 e R7 reproduziram com maior fidelidade os sobre-sinais do sistema completo quando submetido a uma entrada degrau.

A partir da figura 57, observa-se que a métrica $\mathcal{M}_{s s}$ dos modelos reduzidos pelas técnicas R6 e R7 é menor que $10^{-9}$ no intervalo entre 20 a $200 \mathrm{GDL}$ eliminados. Isso significa que no intervalo citado, os modelos reduzidos pelas técnicas R6 e R7 reproduziram os sobre-sinais do sistema completo com erro relativo médio inferior a $10^{-7 \%}$ (Onde o erro relativo médio dos sobre-sinais é definido pela métrica $\mathcal{M}_{s s}$, que é igual à raiz da soma dos quadrados dos erros dos sobre-sinais do modelo reduzido, conforme a equação 172).

De acordo com as figuras 53 a 57 observa-se que os valores das métricas de representatividade não são estritamente crescentes com o aumento do número de GDL eliminados. Em outras palavras, existe uma tendência de que os valores das métricas de erro aumentem conforme o aumento do número de GDL eliminados, entretanto existem situações onde pode haver a diminuição do erro do modelo reduzido com o aumento do número de GDL reduzidos.

Como exemplo, na figura 53, a métrica $\mathcal{M}_{\mathcal{L}_{2}}$ dos modelos reduzidos pela técnica R2 aumentou quando o número de GDL eliminados passou de 270 para 320. Entretanto seu valor passou a diminuir quando aumentamos o número de GDL eliminados até 340.

Apesar disso, o leitor pode observar que existe uma tendência global de que os valores dos erros aumentem com o aumento do número de GDL eliminados, o que pode ser observado nas figuras 53 a 57.

\subsubsection{Representatividade total dos modelos reduzidos}

No item 12.5 .2 será calculada a representatividade total dos modelos reduzidos, em função do número de GDL eliminados.

De acordo com o método para cálculo da representatividade total de um modelo reduzido, descrito no item 9.5, é necessário escolher as métricas que quantificam as características do modelo completo que devem ser reproduzidas de modo mais fidedigno pelo modelo reduzido.

As características escolhidas pelo autor são:

- Capacidade de aproximar os sobre-sinais do modelo completo.

- Capacidade de aproximar a resposta $y(t)$ do modelo completo no intervalo de tempo $\left[t_{0}, t_{f}\right]$, com $t_{0}=0 \mathrm{~s}$ e $t_{f}=50 \mathrm{~s}$.

- Capacidade de aproximar a norma $\mathcal{H}_{\infty}$ da função de transferência do modelo completo. 
Desta maneira, o vetor de métricas $\mathcal{M}$, que será utilizado na equação 191 é:

$$
\mathcal{M}=\left[\mathcal{M}_{s s} ; \mathcal{M}_{\mathcal{L}_{2}} ; \mathcal{M}_{\mathcal{H}_{\infty}}\right]^{T}
$$

A matriz de ponderação $P$ definida pelo analista, de acordo com as características que ele deseja priorizar no modelo reduzido, é $P=\operatorname{diag}(3 ; 2 ; 1)$. Isso significa que a primeira métrica do vetor $\mathcal{M}$ tem maior importância para a quantificação do desempenho do modelo reduzido. A segunda métrica do vetor tem importância intermediária e a terceira métrica tem menor relevância na quantificação do desempenho do modelo reduzido.

A figura 58 mostra os valores da representatividade total $\mathcal{R}$ dos modelos reduzidos, calculados segundo a equação 191 . em função do número de GDL eliminados.

Figura 58 - Representatividade total $\mathcal{R}$ em função do número de GDL reduzidos

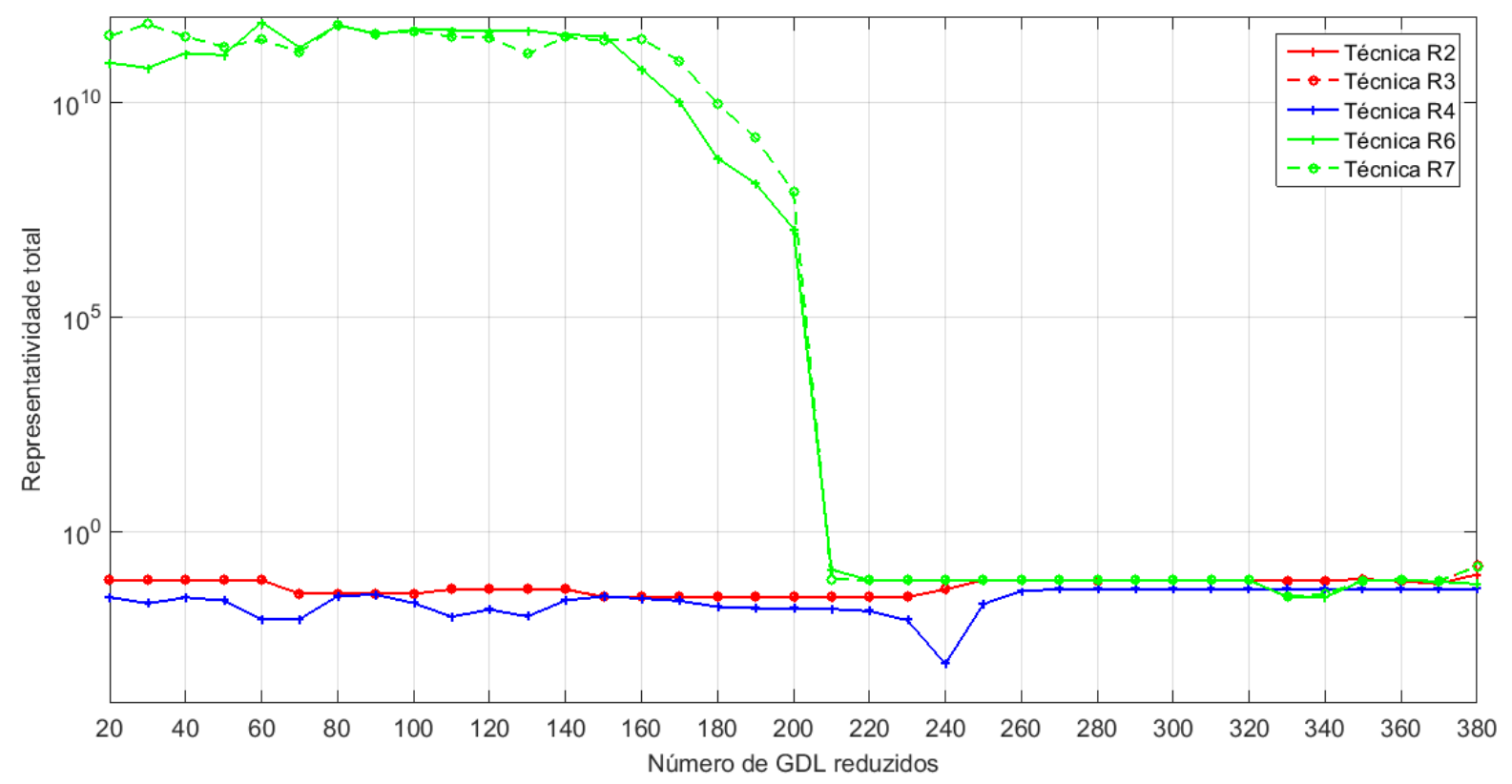

Vale lembrar que quanto maior a representatividade total $\mathcal{R}$ de um modelo reduzido, meIhor é seu desempenho. Ou seja, melhor é sua capacidade de reproduzir um conjunto de características do modelo completo arbitrariamente definidas.

Conforme a figura 58 , o desempenho dos modelos reduzidos que utilizam a base balanceada é consideravelmente maior no intervalo de 20 a 200 GDL eliminados. Quando aumentamos o número de GDL eliminados acima de 200 , os valores da representatividade total dos modelos em questão cai drasticamente.

Observa-se que a representatividade total dos modelos que utilizam a base balanceada possuem melhor desempenho em relação aos outros analisados, quando são eliminados entre $20 \mathrm{e}$ 240 GDL. Já no intervalo entre 250 e 380 GDL reduzidos, nenhuma das técnicas implementadas apresenta vantagem significativa sobre as demais. 
A figura 59 mostra a representatividade total $\mathcal{R}$ dos modelos reduzidos, no intervalo entre 220 e 380 GDL eliminados.

Figura 59 - Representatividade total $\mathcal{R}$ em função do número de GDL reduzidos (detalhe)

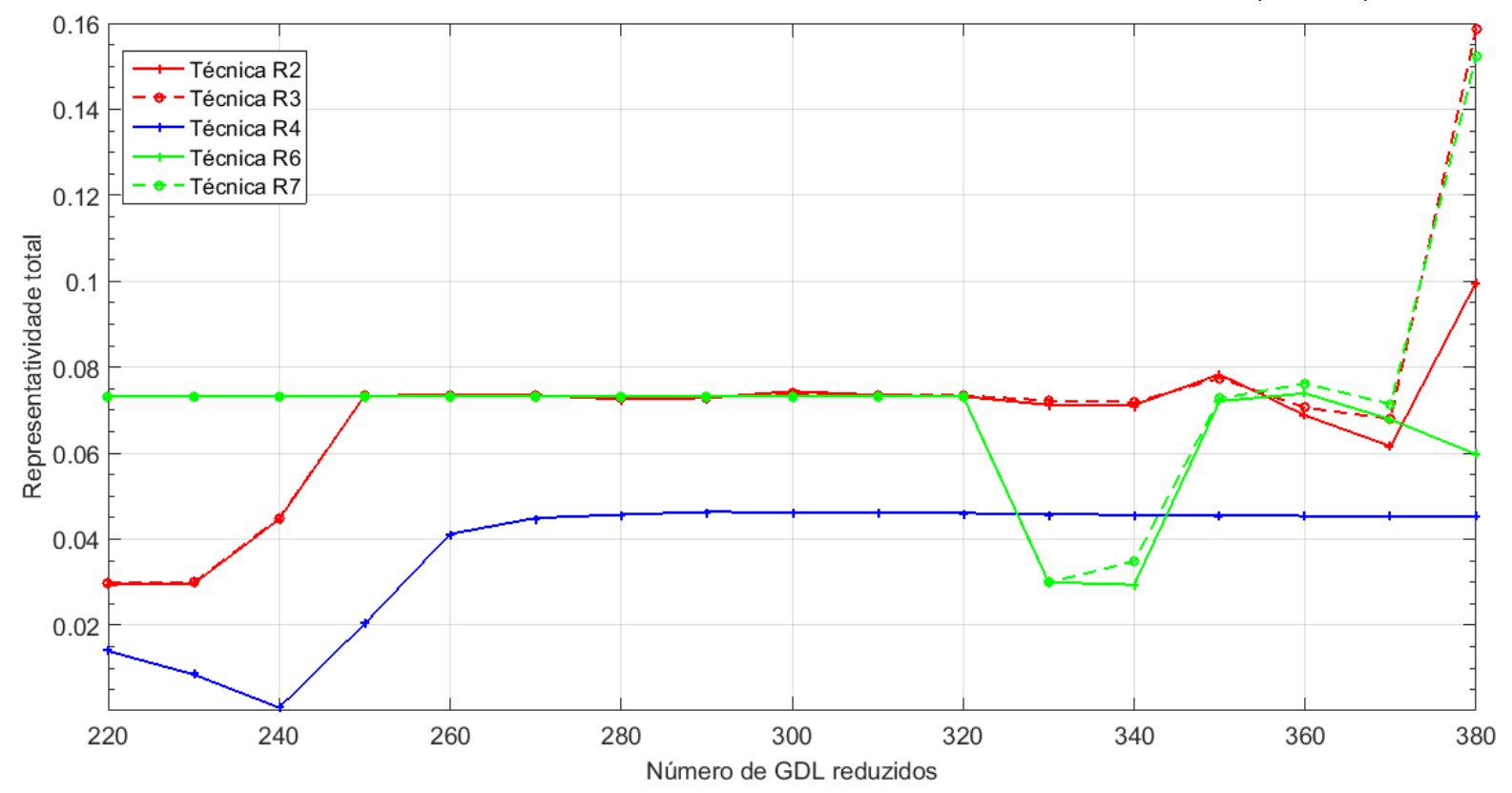

No intervalo entre 220 e $380 \mathrm{GDL}$ eliminados, os modelos reduzidos pelas técnicas R2 e R3 (que utilizam a BFM) tiveram melhor desempenho que os modelos que utilizaram a base DVS.

Observa-se nas figuras 58 e 59 que os valores da representatividade total $\mathcal{R}$ dos modelos reduzidos não são estritamente decrescentes com o aumento do número de GDL eliminados.

\subsubsection{Seleção da técnica mais adequada para a redução do modelo de trem}

Como conclusão do estudo da influência do número de GDL eliminados na representatividade dos modelos reduzidos, o autor irá selecionar a técnica mais adequada para a redução do modelo de trem descrito no item 10.2

Na figura 58 observa-se que os modelos reduzidos pelas técnicas $R 6$ e $R 7$ possuem maior desempenho que os demais no intervalo entre 20 e $200 \mathrm{GDL}$ eliminados.

Também pode-se perceber uma queda abrupta no desempenho dos modelos reduzidos pelas técnicas R6 e R7 quando são eliminados 210 ou mais GDL. Portanto ao realizar a redução de mais de 200 GDL utilizando as técnicas R6 ou R7, o analista irá gerar modelos reduzidos com desempenho consideravelmente menor (comparados à eliminação de 200 GDL ou menos). Desta maneira, o intervalo entre 20 e 200 GDL eliminados será considerado de maior interesse para a elaboração de um modelo reduzido de trem. 
Observa-se na figura 58 que no intervalo entre 20 e $150 \mathrm{GDL}$ eliminados, os modelos reduzidos pelas técnicas $\mathrm{R} 6$ e $\mathrm{R} 7$ possuem desempenho muito próximo, não apresentando indícios de uma vantagem significativa de uma técnica em relação à outra. Já no intervalo entre 160 e $200 \mathrm{GDL}$, a técnica R7 possui vantagem sobre as demais pois está associada a modelos reduzidos com desempenho superior aos demais analisados.

Portanto a técnica R7 é a mais indicada para a elaboração de modelos reduzidos de trem no intervalo de interesse (entre 20 e $200 \mathrm{GDL}$ eliminados).

Como conclusão, o autor elegeu o modelo reduzido pela técnica R7 com 200 GDL eliminados como o mais indicado para representar o modelo completo de trem. Cabe lembrar que o modelo completo tem $398 \mathrm{GDL}$ e o modelo reduzido em questão possui 198, representando uma redução de mais de $50 \%$ no número de GDL.

Abaixo foram elencadas algumas características referentes à representatividade do modelo reduzido selecionado.

- Aproxima a norma $\mathcal{L}_{2}$ (avaliada no intervalo $t \in[0,50]$ segundos) do modelo completo com erro relativo de $6,910^{-9} \%$ (considerando uma entrada degrau), conforme a equação 175.

- Aproxima a norma $\mathcal{H}_{\infty}$ do modelo completo com erro relativo de $9,110^{-9} \%$, conforme a equação 183 .

- Aproxima com exatidão todas as respostas estáticas do modelo completo.

- Aproxima os sobre-sinais do modelo completo com erro relativo médio de 7, $010^{-7 \%}$ (Onde o erro relativo médio dos sobre-sinais é definido pela métrica $\mathcal{M}_{s s}$, que é igual à raiz da soma dos quadrados dos erros dos sobre-sinais do modelo reduzido, conforme a equação 172. 


\section{DISCUSSÕES DOS RESULTADOS}

No capítulo 11, foi mostrado que as técnicas R2, R4, R6 e R7 apresentadas na tabela 5 permitem que o analista determine o número de GDL que serão eliminados do sistema completo. Esta vantagem foi utilizada no item 12.3.1 para elaboração de modelos reduzidos a partir do modelo completo de veículo de passeio. A determinação do número de GDL eliminados foi realizada através:

- De uma aproximação da norma $\left\|G_{e}\right\|_{\mathcal{H}_{\infty}}$ em função do número de GDL eliminados (conforme a equação 212). Para o modelo reduzido pela técnica R2

- Do máximo valor singular da diferença entre as matrizes $A$ dos sistemas completo e reduzido (conforme equação 223) em função do número de GDL eliminados. Para o modelo reduzido pela técnica $\mathrm{R} 4$

- De um limite superior da norma $\left\|G_{e}\right\|_{\mathcal{H}_{\infty}}$ em função do número de GDL eliminados (conforme a equação 228). Para modelos reduzidos pelas técnicas R6 e R7

O autor salienta a vantagem em se utilizar as técnicas de redução R2, R4, R6 e R7 pois possibilitam que o analista possa estimar o número de GDL a serem eliminados antes que seja calculado o modelo reduzido.

Nos itens 12.3.1 e 12.5 o autor relatou que foram encontrados modelos reduzidos mal condicionados durante a aplicação da técnica R5, que utiliza a base BKMA. A possibilidade da existência de sistemas reduzidos mal condicionados pela técnica R5 também foi relatada por Antoulas (ANTOULAS; SORENSEN; GUGERCIN, 2001).

Os resultados apresentados no capítulo 12 constataram que:

- Os modelos reduzidos que utilizaram o método EPS para a eliminação de GDL tiveram erro estático nulo, conforme previsto na equação 147

- Os modelos reduzidos que utilizaram o método truncamento direto para a eliminação de GDL tiveram erro $G_{e}(\infty)=0$, conforme previsto na equação 138 .

O uso das métricas de representatividade descritas no capítulo 9 possibilitou que o autor realizasse nos itens 12.3.4, 12.4 e 12.5 .1 comparações quantitativas entre os resultados dos modelos reduzidos. Com isso, o autor determinou com clareza quais são os modelos que reproduzem com maior fidelidade as características do modelo completo correspondentes a cada métrica analisada. 
No item 12.3.5, o método proposto pelo autor para cálculo da representatividade total possibilitou que fosse eleito o modelo que melhor reproduzia o conjunto de características do modelo completo previamente definidas. Neste caso, o modelo eleito foi o MR3.

A utilização do método para cálculo da representatividade total possibilitou determinar a influência do número de GDL eliminados no desempenho de um modelo reduzido no item 12.5.3. Com isso, foi possível selecionar de maneira quantitativa a técnica mais adequada para a redução do modelo de trem. Neste caso, a técnica selecionada foi a R7.

Um modelo reduzido com 198 GDL utilizando a técnica R7 foi elaborado a partir do modelo completo de trem (que possui $398 \mathrm{GDL}$ ). Com a redução de mais de $50 \%$ no número de GDL do sistema completo, os erros do modelo reduzido apresentados no item 12.5 .3 são bastante satisfatórios. 


\section{CONCLUSÕES}

Neste trabalho, foi apresentada uma revisão sobre técnicas de redução da ordem de modelos dinâmicos lineares e invariantes no tempo. O objetivo da implementação de tais técnicas é a reprodução de características da resposta de um modelo de alta ordem, através de um modelo de ordem reduzida com menor número de graus de liberdade.

Ao longo do texto, foi discutida a capacidade que um modelo reduzido tem de reproduzir características da resposta do completo, que depende de fatores como:

- Base em que o modelo é projetado para que seja realizado a redução de GDL

- Ordem de prioridade de seleção dos GDL a serem eliminados

- Quantidade de graus de liberdade eliminados

- Método de eliminação de GDL utilizado

- Método de correção do modelo reduzido

Foi apresentada uma revisão sobre técnicas para realizar projeções do sistema em diferentes bases, selecionar os GDL que são bons candidatos a eliminação, eliminar GDL do modelo completo e implementar correções na resposta do modelo reduzido.

O autor também destacou a existência de técnicas de redução que possibilitam o analista determinar o número de GDL a serem eliminados antes que o modelo reduzido seja calculado:

- Técnicas de redução que utilizam a projeção do sistema na base modal, com eliminação por truncamento direto e sem implementação de métodos de correção.

- Técnicas de redução que utilizam a projeção do sistema na base de vetores singulares, com eliminação por truncamento direto e sem implementação de métodos de correção.

- Técnicas de redução que utilizam a projeção do sistema na base de realização balanceada, com eliminação por truncamento direto, EPS, EPSG ou EPSGP e sem implementação de métodos de correção.

Com o objetivo de avaliar a fidelidade com que um modelo reduzido reproduz a resposta do modelo de alta ordem, o autor apresentou maneiras de quantificar as similitudes entre as respostas dos modelos completo e reduzido através das métricas de representatividade. A utilização de tais métricas possibilitou que o autor realizasse comparações quantitativas entre os resultados dos modelos reduzidos. 
No item 9.4, o autor descreveu conflitos que podem ocorrer durante a seleção do modelo reduzido mais adequado. Foi mostrado que o método de quantificação de desempenho proposto pelo autor apresenta vantagem sobre a utilização das métricas de representatividade, evitando tais conflitos.

A quantificação do desempenho do modelo reduzido, que é feita pelo cálculo da representatividade total $\mathcal{R}$, possibilita ao analista determinar quantitativamente o modelo que melhor aproxima um conjunto de características previamente estabelecidas do modelo completo.

Foram discutidas e relatadas limitações que o autor encontrou ao implementar algumas das técnicas de redução, como a possibilidade que o modelo reduzido seja não controlável, não observável, instável ou mal condicionado.

De acordo com os resultados apresentados no capítulo 12, pode-se observar que as características que o modelo reduzido representará com maior fidelidade dependem da técnica de redução escolhida.

Como contribuição deste trabalho, o autor propôs métricas para mensurar a capacidade do modelo reduzido em reproduzir as seguintes características:

- Máximos sobre-sinais do SC para uma entrada degrau (através da métrica $\mathcal{M}_{s s}$ ).

- Tempos de acomodação do SC para uma entrada degrau (através da métrica $\mathcal{M}_{s}$ ).

- Norma $\mathcal{L}_{2}$ da resposta do SC (através da métrica $\mathcal{M}_{\mathcal{L}_{2}}$ ).

- Norma $\mathcal{H}_{2}$ da resposta do SC (através da métrica $\mathcal{M}_{\mathcal{H}_{2}}$ ).

- Norma $\mathcal{H}_{2}$ da resposta do SC (através da métrica $\mathcal{M}_{\mathcal{H}_{\infty}}$ ).

Também foram feitas contribuições introduzindo técnicas que possibilitam a correção da resposta estática e do máximo sobre-sinal de modelos reduzidos nos itens 8.2 e 8.3 respectivamente.

No item 9.5 autor propôs uma nova definição para o desempenho de um modelo reduzido, que é sua capacidade de reproduzir um conjunto de características do modelo completo arbitrariamente definidas.

Outra contribuição do autor foi um método para quantificar o desempenho de um modelo reduzido. Neste método, o desempenho de um modelo reduzido é determinado por um valor escalar, chamado de representatividade total $\mathcal{R}$ do modelo. 


\section{TRABALHO FUTURO}

Como foi mostrado neste trabalho, a determinação de aproximações ou de limites superiores das métricas de representatividade antes que o modelo reduzido seja calculado é uma grande vantagem no processo de redução. Portanto o autor destaca a importância de que novos avanços nesta área sejam realizados.

Segundo a literatura pesquisada, a determinação de limites superiores para as taxas de decaimento das normas Hankel de um sistema dinâmico linear ainda é um assunto pouco explorado.

A literatura existente sobre a capacidade que um modelo reduzido tem de aproximar a fase do modelo completo nos diagramas de Bode é escassa, sugerindo maior investigação neste tema. 


\section{Referências}

ALLEYNE, A. Improved vehicle performance using combined suspension and braking forces. Vehicle System Dynamics, Taylor \& Francis, v. 27, n. 4, p. 235-265, 1997.

ANTOULAS, A. C. Approximation of large-scale dynamical systems. [S.I.]: Siam, 2005. v. 6. DOI: $10.1137 / 1.9780898718713$.

ANTOULAS, A. C.; SORENSEN, D. C.; GUGERCIN, S. A survey of model reduction methods for large-scale systems. Contemporary mathematics, Providence, RI; American Mathematical Society; 1999, v. 280, p. 193-220, 2001.

ANTOULAS, A. C.; SORENSEN, D. C.; ZHOU, Y. On the decay rate of hankel singular values and related issues. Systems \& Control Letters, Elsevier, v. 46, n. 5, p. 323-342, 2002. DOI: $10.1016 /$ S0167-6911(02)00147-0.

ARNOLDI, W. E. The principle of minimized iterations in the solution of the matrix eigenvalue problem. Q. Appl. Math, v. 9, n. 17, p. 17-29, 1951.

ARORA, S.; BARAK, B. Computational complexity: a modern approach. [S.I.]: Cambridge University Press, 2009.

BAI, Z. Krylov subspace techniques for reduced-order modeling of large-scale dynamical systems. Applied Numerical Mathematics, Elsevier, v. 43, n. 1, p. 9-44, 2002. DOI: doi:10.1016/S0168-9274(02)00116-2.

BAMPTON, M. C.; CRAIG JR, R. R. Coupling of substructures for dynamic analyses. AIAA Journal, v. 6, n. 7, p. 1313-1319, 1968.

BARBOSA, R.; COSTA, A. Dinâmica do rodeiro ferrovi: Revista Brasileira de Ciências Mecânicas-ABCM, v. 18, n. 4, p. 318-329, 1996.

BARBOSA, R. S. Estudo da dinâmica longitudinal do trem. 1993. Dissertação (Dissertação (Mestrado em Engenharia Mecânica)) — Universidade Estadual de Campinas.

BENDER, C. M.; ORSZAG, S. A. Advanced mathematical methods for scientists and engineers I: Asymptotic methods and perturbation theory. [S.I.]: Springer Verlag, 1999. v. 1.

BENNER, P.; MEHRMANN, V.; SORENSEN, D. C. Dimension reduction of large-scale systems. [S.I.]: Springer, 2005. v. 45. 
BESSELINK, B. et al. A comparison of model reduction techniques from structural dynamics, numerical mathematics and systems and control. Journal of Sound and Vibration, Elsevier, v. 332, n. 19, p. 4403-4422, 2013. DOI: 10.1016/j.jsv.2013.03.025.

CHATURVEDI, D. K. Modeling and simulation of systems using MATLAB and Simulink. [S.I.]: CRC Press, 2011.

CHERRY, A.; COSTA, A.; JONES, R. Mbs modelling, non-linear simulation and linear analysis techniques for integrated vehicle control. Proceedings of IFAC Workshop on Advances in Automotive Control. p. 76-81, 1995.

COOK, R. D. Finite element modeling for stress analysis. [S.I.]: Wiley, 1994.

COSTA, I. Application of multibody systems techniques to vehicle modelling. Model Building Aids for Dynamic System Simulation, IEE Colloquium on. p. 4-1, 1991.

COSTA, I. Application of multibody system (MBS) techniques to automotive vehicle chassis simulation for motion control studies. 1992. Tese (Doutorado) - University of Warwick.

COSTA, I.; JONES, R. Automotive Vehicle Chassis Simulation for Motion Control Studies Using Multibody Systems (MBS) ModellingTechniques. [S.I.: s.n.], 1992.

COSTA, I.; JONES, R. Modelling of automotive vehicles for motion control studies. In: Computational dynamics in multibody systems. Computational dynamics in multibody systems. [S.I.]: Springer, 1995. p. 197-217.

CRAIG, R. Substructure methods in vibration. Journal of Vibration and Acoustics, American Society of Mechanical Engineers, v. 117, n. B, p. 207-213, 1995. DOI: 10.1115/1.2838665.

CRAIG, R. R. Coupling of substructures for dynamic analyses: an overview. Proceedings of AIAA/ASME/ASCE/AHS/ASC structures, structural dynamics, and materials conference and exhibit. p. 1573-1584, 2000.

DAVISON, E. Further remarks on simplifying linear dynamic systems. Automatic Control, IEEE Transactions on, IEEE, v. 12, n. 2, p. 213-214, 1967. DOI: 10.1109/TAC.1967.1098557.

ERSAL, T. et al. A review of proper modeling techniques. Journal of Dynamic Systems, Measurement, and Control, v. 130, n. 6, p. 61008, 2008. DOI: 10.1115/1.2977484.

ERSAL, T. et al. Model reduction in vehicle dynamics using importance analysis. Vehicle System Dynamics, Taylor \& Francis, v. 47, n. 7, p. 851-865, 2009. DOl: $10.1080 / 00423110802444071$. 
FALLAH, M.; BHAT, R.; XIE, W. New model and simulation of macpherson suspension system for ride control applications. Vehicle System Dynamics, Taylor \& Francis, v. 47, n. 2, p. 195-220, 2009. DOI: 10.1080/00423110801956232.

FEHR, J.; EBERHARD, P. Simulation process of flexible multibody systems with non-modal model order reduction techniques. Multibody System Dynamics, Springer, v. 25, n. 3, p. 313-334, 2011. DOI: 10.1007/s11044-010-9238-3.

FELDMANN, P.; FREUND, R. W. Efficient linear circuit analysis by padé approximation via the lanczos proce. Computer-Aided Design of Integrated Circuits and Systems, IEEE Transactions on, IEEE, v. 14, n. 5, p. 639-649, 1995. DOI: 10.1109/43.384428.

FERRAZ, F. G. Detecção de Falhas em Estruturas Complexas usando Síntese Modal dos Componentes e Vetores de Ritz. 2001. Dissertação (Mestrado) - Faculdade de Engenharia Mecânica da Universidade Estadual de Campinas.

FISCHER, M.; EBERHARD, P. Linear model reduction of large scale industrial models in elastic multibody dynamics. Multibody System Dynamics, Springer, v. 31, n. 1, p. 27-46, 2014. DOI: $10.1007 /$ s11044-013-9347-x.

FOSSARD, A. On a method for simplifying linear dynamic systems. Automatic Control, IEEE Transactions on, IEEE, v. 15, n. 2, p. 261-262, 1970. DOI: 10.1109/TAC.1970.1099420

FU, Z.-F.; HE, J. Modal analysis. [S.I.]: Butterworth-Heinemann, 2001.

GAWRONSKI, W. K. Dynamics and control of structures: A modal approach. [S.I.]: Springer New York, 1998.

GIVOLI, D.; BARBONE, P. E.; PATLASHENKO, I. Which are the important modes of a subsystem? International journal for numerical methods in engineering, Wiley Online Library, v. 59, n. 12, p. 1657-1678, 2004. DOI: 10.1002/nme.935.

GLOVER, K. All optimal hankel-norm approximations of linear multivariable system and their I-infinity error bounds. International Journal of Control, v. 39, p. 1115-1193, 1984. DOI: 10.1080/00207178408933239.

GOLDSTEIN, H. Classical mechanics. [S.I.]: Pearson Education India, 1962. v. 4.

GOLUB, G. H.; LOAN, C. F. Matrix computations. [S.I.]: JHU Press, 1996. v. 3.

GREENWOOD, D. Principles of Dynamics, the 2nd Edition. [S.I.]: Englewood Cliffs, NJ Prentice-Hall, 1988. 
GREGORY, C. Reduction of large flexible spacecraft models using internal balancing theory. Journal of Guidance, Control, and Dynamics, v. 7, n. 6, p. 725-732, 1984. DOl: $10.2514 / 3.19919$.

GRIMME, E. J. Krylov projection methods for model reduction. 1997. Tese (Doutorado) - University of Illinois.

GRIMME, E. J.; SORENSEN, D. C.; DOOREN, P. Model reduction of state space systems via an implicitly restarted lanczos method. Numerical Algorithms, Springer, v. 12, n. 1, p. 1-31, 1996. DOI: 10.1007/BF02141739.

GUGERCIN, S.; ANTOULAS, A. C. A survey of model reduction by balanced truncation and some new results. International Journal of Control, Taylor \& Francis, v. 77, n. 8, p. 748-766, 2004. DOI: 10.1080/00207170410001713448.

GUGERCIN, S.; ANTOULAS, A. C.; BEATTIE, C. H2 model reduction for large-scale linear dynamical systems. SIAM journal on matrix analysis and applications, SIAM, v. 30, n. 2, p. 609-638, 2008. DOI: 10.1137/060666123.

GUYAN, R. J. Reduction of stiffness and mass matrices. AIAA journal, v. 3, n. 2, p. 380-380, 1965. DOI: $10.2514 / 3.2874$.

HINCH, E. Perturbation methods. [S.I.]: Cambridge University Press, 1991. v. 6.

HIRSCH, M. W.; DEVANEY, R. L.; SMALE, S. Differential equations, dynamical systems, and linear algebra. [S.I.]: Academic press, 1974. v. 60.

HONG, K.-S.; JEON, D.-S.; SOHN, H.-C. A new modeling of the macpherson suspension system and its optimal pole-placement control. Proc. of the 7th Mediterranean Conference on Control and Automation. p. 559-579, 1999.

HOWITT, G. D.; LUUS, R. Model reduction by minimization of integral square error performance indices. Journal of the Franklin Institute, Elsevier, v. 327, n. 3, p. 343-357, 1990. DOI: 10.1016/0016-0032(90)90001-Y.

IMRAN, M.; GHAFOOR, A.; SREERAM, V. A frequency weighted model order reduction technique and error bounds. Automatica, Elsevier, v. 50, n. 12, p. 3304-3309, 2014. DOI: 10.1016/j.automatica.2014.10.062.

JAIMOUKHA, I. M.; KASENALLY, E. M. Implicitly restarted krylov subspace methods for stable partial realizations. SIAM Journal on Matrix Analysis and Applications, SIAM, v. 18, p. 633-652, 1997. DOI: 10.1137/S0895479895279873.

JAZAR, R. N. Vehicle dynamics: theory and application. [S.I.]: Springer Science \& Business Media, 2013. 
JR, R. R.; CHANG, C.-j. On the use of attachment modes in substructure coupling for dynamic analysis. transformation, v. 10, n. 4, p. 3, 1968.

KABAMBA, P. Balanced gains and their significance for 12 model reduction. Automatic Control, IEEE Transactions on, IEEE, v. 30, n. 7, p. 690-693, 1985. DOI: 10.1109/TAC.1985.1104017.

KAILATH, T. Linear systems. [S.I.]: Prentice-Hall Englewood Cliffs, NJ, 1980. v. 1.

KOKOTOVIC, P.; KHALI, H. K.; O'REILLY, J. Singular perturbation methods in control: analysis and design. [S.I.]: Society for Industrial and Applied Mathematics, 1987. v. 25 .

LANCZOS, C. An iteration method for the solution of the eigenvalue problem of linear differential and integral operators. United States Governm. Press Office, 1950.

LAY, D. C. Linear algebra and its applications. [S.I.: s.n.], 2003. 124 p. v. 3.

LEE, C. H. et al. Dynamic response analysis of monorail bridges under moving trains and riding comfort of trains. Engineering Structures, Elsevier, v. 27, n. 14, p. 1999-2013, 2005. DOI: doi:10.1016/j.engstruct.2005.06.014.

LEVINSON, N. Perturbations of discontinuous solutions of non-linear systems of differential equations. Acta Mathematica, Springer, v. 82, n. 1, p. 71-106, 1950. DOI: 10.1007/BF02398275.

LIU, Y.; ANDERSON, B. D. Singular perturbation approximation of balanced systems. International Journal of Control, Taylor \& Francis, v. 50, n. 4, p. 1379-1405, 1989. DOI: 10.1080/00207178908953437.

LUUS, R. Optimization in model reduction. International Journal of Control, Taylor \& Francis, v. 32, n. 5, p. 741-747, 1980. DOI: 10.1080/00207178008922887.

MARSHALL, S. An approximate method for reducing the order of a linear system. Control, v. 10, n. 643, p. $1,1966$.

MAZZILLI, C.; MONTICELLI, G.; NETO, N. G. Reduced-order modelling in non-linear dynamics: an approach based on non-linear modes. Proceedings of the Institution of Mechanical Engineers, Part C: Journal of Mechanical Engineering Science, SAGE Publications, v. 225, n. 10, p. 2354-2368, 2011. DOI: 10.1177/0954406211410267.

MAZZILLI, C.; NETO, O. Evaluation of non-linear normal modes for finite-element models. Computers \& structures, Elsevier, v. 80, n. 11, p. 957-965, 2002. DOI: 10.1016/S00457949(02)00061-5. 
MAZZILLI, C. E.; SOARES, M. E.; NETO, O. G. Non-linear normal modes of a simply supported beam: continuous system and finite-element models. Computers \& structures, Elsevier, v. 82, n. 31, p. 2683-2691, 2004. DOI: 10.1016/j.compstruc.2004.07.007.

MEGRETSKI, A. H-infinity model reduction with guaranteed suboptimality bound. American Control Conference, 2006. p. 6-pp, 2006. DOI: 10.1109/ACC.2006.1655397.

MEIROVITCH, L. Computational methods in structural dynamics. [S.I.]: Sijthoff \& Noordhoff International Pub, 1980. v. 5.

MOHAMMADPOUR, J.; GRIGORIADIS, K. M. Efficient modeling and control of largescale systems. [S.I.]: Springer Science \& Business Media, 2010.

MOORE, B. Principal component analysis in linear systems: controllability, observability, and model reduction. Automatic Control, IEEE Transactions on, IEEE, v. 26, n. 1, p. 17-32, 1981. DOI: 10.1109/TAC.1981.1102568.

MUSCATO, G. Parametric generalized singular perturbation approximation for model order reduction. Automatic Control, IEEE Transactions on, IEEE, v. 45, n. 2, p. 339-343, 2000. DOI: $10.1109 / 9.839963$

NARASIMHAMURTHI, N. Inequalities between I-2 and I-infinity norms of strictly proper stable transfer functions. Decision and Control, 1987. 26th IEEE Conference on. v. 26, p. 925-926, 1987. DOI: 10.1109/CDC.1987.272528.

NAYFEH, A. H. Perturbation methods. [S.I.]: Wiley-VCH, 2008.

NAYFEH, A. H.; BALACHANDRAN, B. Applied nonlinear dynamics: analytical, computational and experimental methods. [S.I.]: John Wiley \& Sons, 2008.

NETO, O. G. Redução de graus de liberdade: uma proposta para a análise dinâmica de estruturas aporticadas planas de comportamento não-linear. 1998. Dissertação (Mestrado) - Escola Politécnica da Universidade de São Paulo.

OBINATA, G.; ANDERSON, B. Model Reduction for Control System Design. [S.I.]: Springer Verlag, 2001. v. 1.

OGATA, K. Modern control engineering. 4th ed.. ed. [S.I.]: Prentice-Hall Englewood Cliffs, 2001.

PAZ, M. Practical reduction of structural eigenproblems. Journal of Structural Engineering, American Society of Civil Engineers, v. 109, n. 11, p. 2591-2599, 1983. DOl: 10.1061/(ASCE)0733-9445(1983)109:11(2591). 
PAZ, M. Modified dynamic condensation method. Journal of Structural Engineering, American Society of Civil Engineers, v. 115, n. 1, p. 234-238, 1989. DOl: 10.1061/(ASCE)0733-9445(1989)115:1(234)

PERNEBO, L.; SILVERMAN, L. Model reduction via balanced state space representations. Automatic Control, IEEE Transactions on, IEEE, v. 27, n. 2, p. 382-387, 1982. DOI: 10.1109/TAC.1982.1102945.

RAHROVANI, S.; VAKILZADEH, M. K.; ABRAHAMSSON, T. Modal dominancy analysis based on modal contribution to frequency response function h2-norm. Mechanical Systems and Signal Processing, Elsevier, v. 48, n. 1, p. 218-231, 2014. DOI: doi:10.1016/j.ymssp.2014.03.003.

REGA, G.; TROGER, H. Dimension reduction of dynamical systems: methods, models, applications. Nonlinear Dynamics, Springer, v. 41, n. 1-3, p. 1-15, 2005. DOI: 10.1007/s11071005-2790-3.

RIXEN, D. J. A dual craig-bampton method for dynamic substructuring. Journal of Computational and applied mathematics, Elsevier, v. 168, n. 1, p. 383-391, 2004. DOI: 10.1016/j.cam.2003.12.014.

ROBINSON, C. Dynamical systems: stability, symbolic dynamics, and chaos. [S.I.]: CRC press, 1995.

SAAD, Y. Iterative methods for sparse linear systems. [S.I.]: Society for Industrial and Applied Mathematics, 2003.

SALIMBAHRAMI, B.; LOHMANN, B. Scientific Report. Krylov subspace methods in linear model order reduction: introduction and invariance properties. [S.I.: S.n.], 2002. Disponível em: <http://www.iat.unibremen.de/mitarbeiter/salimbahrami/Invariance.pdf $>$.

SALIMBAHRAMI, B.; LOHMANN, B. Order reduction of large scale second-order systems using krylov subspace methods. Linear Algebra and its Applications, Elsevier, v. 415, p. 385-405, 2006. DOI: 10.1016/j.laa.2004.12.013.

SAMAR, R.; POSTLETHWAITE, I.; GU, D.-W. Applications of the singular perturbation approximation of balanced systems. Control Applications, 1994., Proceedings of the Third IEEE Conference on. p. 1823-1828, 1994. DOI: 10.1109/CCA.1994.381257.

SASTRY, S. Nonlinear systems: analysis, stability, and control. [S.I.]: Springer Science \& Business Media, 2013. v. 10.

SCHILDERS, W. H.; VORST, H. A. Van der; ROMMES, J. Model order reduction: theory, research aspects and applications. [S.I.]: Springer, 2008. v. 13. 
SOARES, M. E. Modos não lineares em sistemas discretizados pelo método de elementos finitos. 1998. Tese (Doutorado) - Escola Politécnica da Universidade de São Paulo.

STEINDL, A.; TROGER, H. Methods for dimension reduction and their application in nonlinear dynamics. International Journal of Solids and Structures, Elsevier, v. 38, n. 10, p. 2131-2147, 2001. DOI: 10.1016/S0020-7683(00)00157-8.

STRANG, G. Linear Algebra and Its Applications. [S.I.]: Thomson, 2006.

VARGA, A. Relatório técnico. On modal techniques for model reduction. [S.I.: s.n.], 1993.

VARGA, A.; ANDERSON, B. D. Accuracy-enhancing methods for balancing-related frequency-weighted model and controller reduction. IEEE Conference on Decision and Control. v. 4, p. 3659-3664, 2001. DOI: doi:10.1016/S0005-1098(03)00030-X.

VARGA, A.; PARRILO, P. Fast algorithms for solving h-infinity norm minimization problems. Decision and Control, 2001. Proceedings of the 40th IEEE Conference on. v. 1, p. 261-266, 2001. DOI: 10.1109/.2001.980109.

WILLIAMS, T.; CHENG, X. Degrees of controllability and observability for close modes of flexible space structures. Automatic Control, IEEE Transactions on, IEEE, v. 44, n. 9, p. 1791-1795, 1999. DOI: 10.1109/9.788555

ZHOU, K.; DOYLE, J. C. Essentials of robust control. [S.I.]: Prentice hall Upper Saddle River, NJ, 1998. v. 104.

ZHOU, K. et al. Robust and optimal control. [S.I.]: Prentice Hall New Jersey, 1996. v. 40. 


\section{Apêndice A - Sistemas multicorpos}

É dado um sistema multicorpos com $N$ elementos, $l N$ variáveis e $k$ equações de restrições. O primeiro corpo pode ser representado pelas variáveis $\left(x_{1}, x_{2}, \ldots, x_{l}\right)$, o segundo por $\left(x_{l+1}, x_{l+2}, \ldots, x_{2 l}\right)$ e assim por diante.

$$
\begin{aligned}
& x_{1}=x_{1}\left(q_{1}, q_{2}, \ldots, q_{l N-k}, t\right) \\
& \vdots \\
& x_{l N}=x_{l N}\left(q_{1}, q_{2}, \ldots, q_{l N-k}, t\right)
\end{aligned}
$$

Onde $q_{i}$ são chamadas variáveis independentes ou generalizadas e $l N-k$ é o número de graus de liberdade do sistema.

Neste caso, estamos considerando que as restrições do sistema são holonômicas.

Para essa consideração, define-se uma restrição holonômica aquela que relaciona as coordenadas do sistema com uma função do tipo $\phi\left(x_{1}, x_{2}, x_{3}, \ldots, t\right)$. Conseqüentemente, o quantidade de coordenadas generalizadas $q_{i}$ é igual ao número de graus de liberdade do sistema, além de que elas são independentes entre si (GOLDSTEIN, 1962). Ou seja,

$$
\frac{\partial q_{i}}{\partial q_{j}}=0 \quad \text { para } \quad i \neq j
$$

As restrições que não relacionam as coordenadas do sistema com uma função do tipo $\phi\left(x_{1}, x_{2}, x_{3}, \ldots, t\right)$ são chamadas não holonômicas. Neste caso, existe pelo menos uma coordenada generalizada $q_{i}$ tal que

$$
\frac{\partial q_{i}}{\partial q_{j}} \neq 0 \quad \text { para } \quad i \neq j
$$

Um exemplo de restrição não holonômica é um disco de espessura desprezível rolando sem escorregar sobre um plano, como mostra a figura 60.

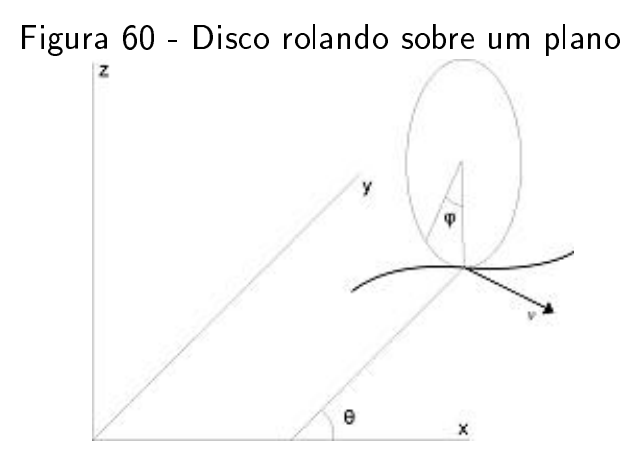

O disco tem raio $r$ e rola no plano $x y$, de maneira que a equação de vínculo será expressa 
pelas velocidades generalizadas.

$$
\begin{gathered}
\dot{x}=r \dot{\varphi} \operatorname{sen} \theta \\
\dot{y}=-r \dot{\varphi} \cos \theta
\end{gathered}
$$

Consequentemente podemos escrever

$$
\dot{x}=-\dot{y} \tan \theta \text {. }
$$

A relação acima não pode ser integrada afim de obter uma equação de vínculo na forma $\phi\left(x_{1}, x_{2}, x_{3}, \ldots, t\right)$. Também se pode perceber que as coordenadas generalizadas não são independentes entre si, como é mostrado na equação 240.

$$
\frac{\partial x}{\partial y}=-\tan \theta
$$

Pelas razões acima citadas, o vínculo em questão é não holonômico.

\section{A.1 MÉTODO DE LAGRANGE}

Para sistema em equilíbrio estático, baseando-se no princípio de deslocamentos virtuais, temos a soma das forças resultantes em um corpo definidas como na equação 241.

$$
\sum_{\text {corpo }} F_{j} \delta x_{j}=\sum_{\text {corpo }}\left(F_{j}^{(e x t)}+f_{j}\right) \delta x_{j}=0
$$

Essas resultantes $F_{j}$ são resultado da soma das forças externas $F_{j}^{(e x t)}$ com as forças de restrições $f_{j}$. Por enquanto ficaremos restritos a sistemas onde o trabalho virtual das forças nas restrições é zero.

Dessa maneira, são definidas as forças generalizadas do sistema (GOLDSTEIN, 1962)

$$
Q_{i}=\sum_{j=1}^{l N} F_{j} \frac{\partial x_{j}}{\partial q_{i}} \quad(i=1,2, \ldots, l N-k)
$$

Assim, o trabalho virtual dessas forças em um deslocamento virtual $\delta W$ arbitrário e consistente com as equações de restrição é

$$
\delta W=\sum_{i} Q_{i} \delta q_{i}=\sum_{i} \sum_{j} F_{j} \frac{\partial x_{j}}{\partial q_{i}} \delta q_{i}=0
$$

De onde pode-se concluir que

$$
\sum_{j} F_{j} \frac{\partial x_{j}}{\partial q_{i}}=0
$$


Da equação acima, a afirmação de que o número de variáveis independentes $q_{i}$ é igual ao número de graus de liberdade dá o lugar para outra definição: O número de variáveis independentes $q_{i}$ é igual ao número de graus de liberdade se a soma das forças generalizadas em cada corpo, (referentes às restrições que não realizam trabalho) for zero (GREENWOOD, 1988) (em outras palavras, se a equação acima for satisfeita).

O princípio de D'Alembert é definido como

$$
\sum_{\text {corpo }}\left(F_{j}^{(e x t)}-\dot{p_{j}}\right) \delta x_{j}=0
$$

sendo $p_{j}$ a projeção do momento linear do corpo na direção $x_{j}$.

Baseada neste princípio, a equação de Lagrange para sistemas conservativos pode ser apresentada na forma

$$
\frac{d}{d t}\left(\frac{\partial L}{\partial \dot{q}_{i}}\right)-\frac{\partial L}{\partial q_{i}}=0
$$

com

$$
L=T-V
$$

onde $T$ e $V$ são respectivamente as equações das energias cinética e potencial no sistema e

$$
\begin{gathered}
V=V\left(q_{1}, q_{2}, \ldots, q_{n}\right) \\
\text { com }-\frac{\partial V}{\partial q_{i}}=Q_{i}
\end{gathered}
$$

No entanto, pode-se considerar a equação da energia potencial como uma equação do tipo

$$
\begin{array}{r}
V=V\left(q_{1}, q_{2}, \ldots, q_{l N-k}, t\right) \\
\operatorname{com} \quad Q_{i}=-\frac{\partial V}{\partial q_{i}}+\frac{d}{d t}\left(\frac{\partial V}{\partial \dot{q}_{i}}\right)
\end{array}
$$

Isso possibilita escrever a equação de Lagrange na forma

$$
\frac{d}{d t}\left(\frac{\partial L}{\partial \dot{q}_{i}}\right)-\frac{\partial L}{\partial q_{i}}=Q_{i}^{\prime}
$$

Onde $Q_{i}^{\prime}$ é a soma das forças generalizadas que não são provenientes de um potencial $V$, por exemplo as forças dissipativas.

Por final, pode-se escrever a equação de Lagrange mais generalizada, considerando sistemas com restrições não holonômicas

$$
\frac{d}{d t}\left(\frac{\partial L}{\partial \dot{q}_{i}}\right)-\frac{\partial L}{\partial q_{i}}=Q_{i}^{\prime}+\sum_{m=1}^{k} \lambda_{m} a_{m, i}
$$


Onde $\lambda_{m}$ são chamados multiplicadores de Lagrange e $a_{m, i}$ dependem das coordenadas generalizadas e do tempo. Dessa maneira, $\sum \lambda_{m} a_{m, i}$ é a soma das forças de restrição generalizadas referentes às coordenadas $q_{i}$ tal que

$$
\sum_{i}\left(\sum_{m=1}^{k} \lambda_{m} a_{m, i}\right) \delta q_{i}=0
$$

Dessa maneira, a equação de Lagrange é uma alternativa mais conveniente de escrever as equações de movimento de um sistema em relação à sua forma original usando leis de Newton. Isso é possível pois trabalhalha-se com grandezas escalares, como as energias cinética e potencial, em substituição à grandezas vetoriais como forças e acelerações.

É importante mencionar que, no conjunto de forças generalizadas $Q_{i}^{\prime}$, existe uma classe de forças que são proporcionais à velocidade relativa entre seus pontos de aplicação e sempre se opõem ao movimento.

$$
F_{j}=-C_{j} \dot{x}_{j}
$$

O trabalho virtual da força analisada pode ser representado por

$$
\delta W=-\sum_{j} C_{j} \dot{x}_{j} \delta x_{j}=-\sum_{i}\left[\sum_{j} \frac{\partial}{\partial \dot{q}_{i}}\left(\frac{C_{j} \dot{x}_{j}^{2}}{2}\right)\right] \delta q_{j}
$$

Dessa maneira, chega-se à função de Rayleigh

$$
R=\sum_{j} \frac{C_{j} \dot{x}_{j}^{2}}{2}
$$

que nos leva a escrever a força generalizada descrita anteriormente como

$$
Q_{i}^{\prime}=-\frac{\partial R}{\partial \dot{q}_{i}}
$$

\section{A.2 EXEMPLO DE APLICAÇÃO DO MÉTODO DE LAGRANGE}

Um modelo de um quarto de veículo com dois graus de liberdade pode ser simplificado de acordo com a figura 61

As equações de energia cinética e potencial são respectivamente

$$
\begin{array}{r}
K=\frac{M_{2} \dot{Z}_{2}^{2}}{2}+\frac{M_{1} \dot{Z}_{1}^{2}}{2} \\
V=\frac{k_{2}\left(Z_{1}-Z_{2}\right)^{2}}{2}+\frac{k_{1}\left(u-Z_{1}\right)^{2}}{2}
\end{array}
$$


Figura 61 - Modelo de um quarto de veículo.

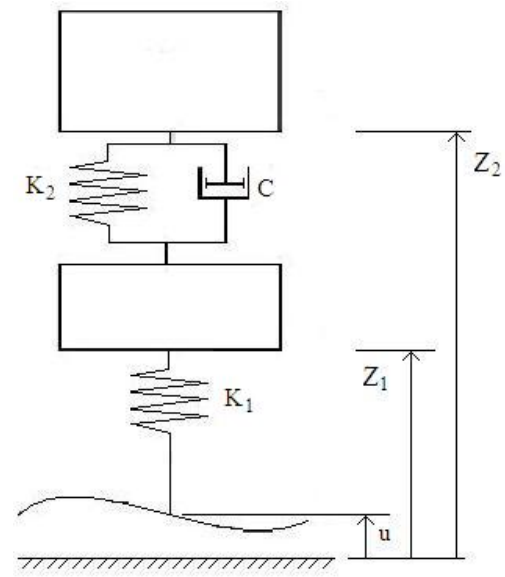

e a Lagrangiana do sistema, de acordo com a equação 247 é

$$
L=\frac{M_{2} \dot{Z}_{2}^{2}}{2}+\frac{M_{1} \dot{Z}_{1}^{2}}{2}-\frac{k_{2}\left(Z_{1}-Z_{2}\right)^{2}}{2}-\frac{k_{1}\left(u-Z_{1}\right)^{2}}{2}
$$

A força no amortecedor é proporcional às velocidades, portanto a função de Rayleigh pode ser escrita como na equação 259 .

$$
R=\frac{C\left(\dot{Z}_{1}-\dot{Z}_{2}\right)^{2}}{2}
$$

Em 260, podemos ver os passos intermediários para o cálculo das equações de movimento.

$$
\begin{array}{r}
\frac{\partial L}{\partial \dot{Z}_{1}}=M_{1} \dot{Z}_{1} \\
\frac{\partial L}{\partial Z_{1}}=-k_{2}\left(Z_{1}-Z_{2}\right)+k_{1}\left(u-Z_{1}\right) \\
\frac{\partial R}{\partial \dot{Z}_{1}}=C\left(\dot{Z}_{1}-\dot{Z}_{2}\right) \\
\frac{\partial L}{\partial \dot{Z}_{2}}=M_{2} \dot{Z}_{2} \\
\frac{\partial L}{\partial Z_{2}}=k_{2}\left(Z_{1}-Z_{2}\right) \\
\frac{\partial R}{\partial \dot{Z}_{2}}=-C\left(\dot{Z}_{1}-\dot{Z}_{2}\right)
\end{array}
$$

Portanto, as equações de movimento do sistema são como as mostradas na 261 .

$$
\begin{array}{r}
M_{1} \ddot{Z}_{1}+k_{2}\left(Z_{1}-Z_{2}\right)-k_{1}\left(u-Z_{1}\right)+C\left(\dot{Z}_{1}-\dot{Z}_{2}\right)=0 \\
M_{2} \ddot{Z}_{2}-k_{2}\left(Z_{1}-Z_{2}\right)-C\left(\dot{Z}_{1}-\dot{Z}_{2}\right)=0
\end{array}
$$


O sistema de equações acima também pode ser escrito na forma de espaço de estados, como apresentado na equação 2

$$
\left(\begin{array}{cccc|c}
0 & 0 & 1 & 0 & 0 \\
0 & 0 & 0 & 1 & 0 \\
-\frac{k_{1}+k_{2}}{M_{1}} & \frac{k_{2}}{M_{1}} & -\frac{C}{M_{1}} & \frac{C}{M_{1}} & \frac{k_{1}}{M_{1}} \\
\frac{k_{2}}{M_{2}} & -\frac{k_{2}}{M_{2}} & \frac{C}{M_{2}} & -\frac{C}{M_{2}} & 0 \\
\hline 0 & 1 & 0 & 0 & 0
\end{array}\right)\left\{\begin{array}{c}
Z_{1} \\
Z_{2} \\
\dot{Z}_{1} \\
\dot{Z}_{2} \\
\hline u
\end{array}\right\}=\left\{\begin{array}{c}
\dot{Z}_{1} \\
\dot{Z}_{2} \\
\ddot{Z}_{1} \\
\ddot{Z}_{2} \\
\hline y
\end{array}\right\}
$$

Avaliando o sistema da equação 262 com os dados de entrada da tabela 17, temos:

$$
\left(\begin{array}{cccc|c}
0 & 0 & 1 & 0 & 0 \\
0 & 0 & 0 & 1 & 0 \\
-741,62 & 72,61 & -13,82 & 13,82 & 669,01 \\
343,67 & -343,67 & 65,40 & -65.40 & 0 \\
\hline 0 & 1 & 0 & 0 & 0
\end{array}\right)\left\{\begin{array}{c}
Z_{1} \\
Z_{2} \\
\dot{Z}_{1} \\
\dot{Z}_{2} \\
\hline u
\end{array}\right\}=\left\{\begin{array}{c}
\dot{Z}_{1} \\
\dot{Z}_{2} \\
\ddot{Z}_{1} \\
\ddot{Z}_{2} \\
\hline y
\end{array}\right\}
$$

Tabela 17 - Dados de entrada para o exemplo de quarto de veículo

\begin{tabular}{|l|c|c|c|}
\hline Parâmetro & Variável & Unidade & Valor \\
\hline Massa de quarto de Chassi & $M_{1}$ & $k g$ & 284 \\
\hline Massa de uma roda & $M_{2}$ & $k g$ & 60 \\
\hline Rigidez do pneu & $k_{1}$ & $\mathrm{~N} / \mathrm{m}$ & 190000 \\
\hline Rigidez da mola & $k_{2}$ & $\mathrm{~N} / \mathrm{m}$ & 20620 \\
\hline Rigidez do amortecedor & $C$ & $\mathrm{~N} /(\mathrm{m} / \mathrm{s})$ & 3924 \\
\hline
\end{tabular}




\section{Apêndice B - Normas de vetores, matrizes e funções}

Neste Apêndice, é apresentada uma breve descrição das normas usadas neste trabalho.

\section{B.1 NORMAS INDUZIDAS}

Uma norma induzida pode ser interpretada como a norma do sinal de saída uma função, normalizada pela norma do sinal de entrada correspondente. Ou seja, é uma medição do ganho entre entrada/saída de uma função (ZHOU et al., 1996).

Dada uma matriz $A \in \mathbb{C}^{n \times m}$, interpretada como um operador linear $A: \mathbb{C}^{m} \rightarrow \mathbb{C}^{n}$, suas normas induzidas $\|A\|_{p-i n d},\|A\|_{2-i n d}$ e $\|A\|_{\infty-i n d}$ são respectivamente:

$$
\begin{aligned}
\|A\|_{p-i n d} & =\sup _{v \neq 0} \frac{\|A v\|_{p}}{\|v\|_{p}} \\
\|A\|_{2-i n d} & =\sup _{v \neq 0} \frac{\|A v\|_{2}}{\|v\|_{2}}=\left[\lambda_{\max }\left(A A^{*}\right)\right]^{\frac{1}{2}}=\left[\lambda_{\max }\left(A^{*} A\right)\right]^{\frac{1}{2}} \\
\|A\|_{\infty-i n d} & =\max _{i \in[1, \ldots, n]} \sum_{j=1}^{m}\left|A_{i j}\right|
\end{aligned}
$$

onde $\lambda_{\max }($.$) é o maior autovalor da matriz em questão e p=1,2, \ldots, \infty$.

\section{B.2 NORMAS NÃO INDUZIDAS}

Dada uma matriz $A \in \mathbb{C}^{n \times m}$, interpretada como um operador linear $A: \mathbb{C}^{m} \rightarrow \mathbb{C}^{n}$, suas normas não induzidas $\|A\|_{p},\|A\|_{2}$ e $\|A\|_{\infty}$ são respectivamente:

$$
\begin{aligned}
\|A\|_{p} & =\left(\sum_{i=1}^{\min (m, n)} \sigma_{i}^{p}(A)\right)^{\frac{1}{p}} \\
\|A\|_{2} & =\left(\sum_{i=1}^{\min (m, n)} \sigma_{i}^{2}(A)\right)^{\frac{1}{2}}=\left[\operatorname{tr}\left(A^{*} A\right)\right]^{\frac{1}{2}} \\
\|A\|_{\infty} & =\sigma_{\max }(A)
\end{aligned}
$$

onde $\operatorname{tr}[],. \sigma_{i}($.$) e \sigma_{\max }($.$) são espectivamente o traço, o i-ésimo valor singular e o máximo$ valor singular da matriz em questão.

Como um vetor $v \in \mathbb{C}^{m}$ pode ser interpretado como uma matriz de uma coluna, a norma $p$ não induzida, descrita acima, pode ser aplicada ao mesmo. Desta maneira, encontramos as relações: 


$$
\begin{aligned}
\|v\|_{p} & =\left(\sum_{i=1}^{m}\left|v_{i}\right|^{p}\right)^{\frac{1}{p}} \\
\|v\|_{2} & =\left(\sum_{i=1}^{m}\left|v_{i}\right|^{2}\right)^{\frac{1}{2}}=\left(v^{*} v\right)^{\frac{1}{2}} \\
\|v\|_{\infty} & =\max _{i \in[1, \ldots, m]}\left|v_{i}\right|
\end{aligned}
$$

\section{B.3 NORMAS DE FUNÇÕES}

Neste trabalho, foram usados dois tipos de espaços lineares distintos $\mathcal{L}_{p}$ (chamados de Espaços de Lebesgue) e $\mathcal{H}_{p}$ (Espaços de Hardy) (ANTOULAS, 2005).

Dada uma função $f(t)$ com $f: \mathbb{R} \rightarrow \mathbb{R}^{n}$ no domínio do tempo, suas normas não induzidas $\mathcal{L}_{p}, \mathcal{L}_{2}$ e $\mathcal{L}_{\infty}$ no intervalo $t \in[a, b]$ são respectivamente

$$
\begin{aligned}
& \|f\|_{\mathcal{L}_{p}[a, b]}=\left(\int_{a}^{b}\|f(t)\|_{p}^{p} d t\right)^{\frac{1}{p}} \\
& \|f\|_{\mathcal{L}_{2}[a, b]}=\left(\int_{a}^{b}\|f(t)\|_{2}^{2} d t\right)^{\frac{1}{2}}=\left(\int_{a}^{b} f^{*}(t) f(t) d t\right)^{\frac{1}{2}} \\
& \|f\|_{\mathcal{L}_{\infty}[a, b]}=\sup _{t \in[a, b]}\|f(t)\|_{\infty}
\end{aligned}
$$

onde $\|f(t)\|_{p}$ é a norma $p$ não induzida de $f(t)$ no instante $t$.

Dada uma função $F(i \omega)$ que é resultado da trasnformada de Laplace de $f(t)$, com $F: \mathbb{C} \rightarrow$ $\mathbb{C}^{n \times m}$ no domínio da frequência. Suas normas não induzidas $\mathcal{L}_{p}, \mathcal{L}_{2}$ e $\mathcal{L}_{\infty}$ são respectivamente

$$
\begin{aligned}
& \|F\|_{\mathcal{L}_{p}}=\left(\sup _{\omega \in \mathbb{R}} \int_{-\infty}^{\infty}\|F(i \omega)\|_{p}^{p} d \omega\right)^{\frac{1}{p}} \\
& \|F\|_{\mathcal{L}_{2}}=\left(\frac{1}{2 \pi} \sup _{\omega \in \mathbb{R}} \int_{-\infty}^{\infty}\|F(i \omega)\|_{2}^{2} d \omega\right)^{\frac{1}{2}} \\
& \|F\|_{\mathcal{L}_{\infty}}=\sup _{\omega \in \mathbb{R}} \sigma_{\max }[F(i \omega)]
\end{aligned}
$$

As normas $\mathcal{L}_{2}$ induzidas de $f(t)$ e $F(i \omega)$ são respectivamente

$$
\begin{aligned}
& \|f\|_{\mathcal{L}_{2-i n d}[a, b]}=\sup _{v \neq 0} \frac{\|f * v\|_{\mathcal{L}_{2}[a, b]}}{\|v\|_{\mathcal{L}_{2}[a, b]}} \\
& \|F\|_{\mathcal{L}_{2-\text { ind }}}=\sup _{V \neq 0} \frac{\|F V\|_{\mathcal{L}_{2}}}{\|V\|_{\mathcal{L}_{2}}}
\end{aligned}
$$

Uma notação com a omissão do intervalo de tempo em que o vetor está sendo analisado 
indica a norma $\mathcal{L}_{2}$ no intervalo $\left[0, \infty\left[\right.\right.$. Por exemplo: $\|f\|_{\mathcal{L}_{2}}=\|f\|_{\mathcal{L}_{2}[0, \infty[},\|f\|_{\mathcal{L}_{2-\text { ind }}}=$ $\|f\|_{\mathcal{L}_{2-\text { ind }}[0, \infty[\text {, etc }}$

É dada uma função $F(i \omega)$ com $F: \mathbb{C} \rightarrow \mathbb{C}^{n \times m}$ no domínio da frequência e com pólos com parte real negativa. Suas normas não induzidas $\mathcal{H}_{2}$ e $\mathcal{H}_{\infty}$ são respectivamente (ZHOU et al., 1996).

$$
\begin{aligned}
\|F\|_{\mathcal{H}_{2}} & =\left(\frac{1}{2 \pi} \int_{-\infty}^{\infty} \operatorname{tr}\left[F^{*}(i \omega) F(i \omega)\right] d \omega\right)^{\frac{1}{2}} \\
\|F\|_{\mathcal{H}_{\infty}} & =\sup _{\omega \in \mathbb{R}} \sigma_{\max }[F(i \omega)]
\end{aligned}
$$

É importante salientar que a seguinte notação será usada: $[F(i \omega)]^{*}=F^{*}(i \omega)$

A norma $\mathcal{H}_{2}$ induzida de $F(i \omega)$ é

$$
\|F\|_{\mathcal{H}_{2-i n d}}=\sup _{V \neq 0} \frac{\|F V\|_{\mathcal{H}_{2}}}{\|V\|_{\mathcal{H}_{2}}}
$$

\section{B.4 CORRELAÇÃO ENTRE NORMAS INDUZIDAS E NÃO INDUZIDAS}

- Normas de matrizes

$$
\|A\|_{\infty}=\|A\|_{2-i n d}
$$

- Normas de funções

Serão apresentadas abaixo, correlações para normas de funções no domínio da frequência.

$$
\begin{aligned}
\|f\|_{\mathcal{L}_{\infty}} & =\|f\|_{\mathcal{L}_{2-i n d}} \\
\|F\|_{\mathcal{L}_{\infty}} & =\|F\|_{\mathcal{L}_{2-i n d}} \\
\|F\|_{\mathcal{H}_{\infty}} & =\|F\|_{\mathcal{H}_{2-i n d}}
\end{aligned}
$$

\section{B.5 CORRELAÇÃO ENTRE NORMAS NO DOMÍNIO DO TEMPO E DA FREQUÊNCIA}

Existe uma relação entre os espaços $\mathcal{L}_{2}$ definidos para o domínio do tempo e no domínio da frequência, que é conhecida como relações de Parseval. Estas relações podem ser encontradas 
com mais detalhes em (ZHOU; DOYLE, 1998).

$$
\begin{aligned}
& \|f\|_{\left.\mathcal{L}_{2}\right]-\infty, \infty[}=\|F\|_{\mathcal{L}_{2}} \\
& \|f\|_{\mathcal{L}_{2}}=\|f\|_{\mathcal{L}_{2}[0, \infty[}=\|F\|_{\mathcal{H}_{2}} \\
& \|f\|_{\mathcal{L}_{2-\text { ind }}}=\sup _{v \neq 0} \frac{\|f * v\|_{\mathcal{L}_{2}}}{\|v\|_{\mathcal{L}_{2}}}=\sup _{V \neq 0} \frac{\|F V\|_{\mathcal{H}_{2}}}{\|V\|_{\mathcal{H}_{2}}}=\|F\|_{\mathcal{H}_{2-i n d}}
\end{aligned}
$$

Onde $f * v$ representa o operador de convolução de $f(t)$ e $v(t)$.

\section{B.6 CORRELAÇÃO ENTRE NORMAS NOS ESPAÇOS $\mathcal{L}$ E $\mathcal{H}$}

Conforme relações demonstradas por Antoulas (ANTOULAS, 2005), as seguintes relações são válidas para sistemas lineares invariantes no tempo.

$$
\begin{aligned}
& \|G\|_{\mathcal{L}_{2}}=\|G\|_{\mathcal{H}_{2}} \\
& \|G\|_{\mathcal{L}_{\infty}}=\|G\|_{\mathcal{H}_{\infty}}
\end{aligned}
$$




\section{Apêndice C - Exemplos numéricos}

Este apêndice contém esemplos numéricos de técnicas utilizadas neste trabalho, as quais são: projeção de um sistema linear em uma base de vetores singulares, projeção de um sistema linear em uma base de Krylov obtida pelo Método de Arnoldi, eliminação de GDL em um sistema pelos métodos de Guyan e Guyan Modificado e correção da resposta estática de um sistema reduzido.

\section{C.1 EXEMPLO NUMÉRICO: DECOMPOSIÇÃO EM VALORES SINGULARES}

Como exemplo da Decomposição em Valores Singulares apresentada no item 5.2 será realizada abaixo a diagonalização da matriz $A$ em valores singulares e apresentados seus respectivos vetores singulares à esquerda e à direita.

$$
A=\left[\begin{array}{cccc}
0,15 & 1,04 & 0,09 & 0,83 \\
0,12 & 1,99 & 0,04 & 0,96 \\
-4,0 & 3,48 & 0,09 & -4,44 \\
0,1 & 6,99 & 0,14 & -0,21
\end{array}\right]
$$

O produto $A A^{*}$ da equação 67 é:

$$
A A^{*}=\left[\begin{array}{cccc}
1,801 & 2,888 & -0,658 & 7,123 \\
& 4,898 & 2,186 & 13,726 \\
& & 47,832 & 24,87 \\
\text { sim } & & & 48,934
\end{array}\right]
$$

Os autovalores de $A A^{*}$ são $\left[\lambda_{1}, \ldots, \lambda_{4}\right]=\left[\begin{array}{llll}75,491 & 27,44 & 0,5309 & 0,00298\end{array}\right]$. Calculando a raiz quadrada destes autovalores, encontramos os valores singulares de $A\left[\sigma_{1}, \ldots, \sigma_{4}\right]=$ $[8,6885 \quad 5,2383 \quad 0,7286 \quad 0,0546]$.

Segundo a equação 67, a matriz cujas colunas são formadas pelos autovetores normalizados de $A A^{*}$ é:

$$
U_{v s}=\left[\begin{array}{cccc}
0,0707 & -0,2131 & -0,5763 & 0,7858 \\
0,1646 & -0,307 & -0,7064 & -0,6161 \\
0,6641 & 0,7276 & -0,1717 & 0,0117 \\
0,7259 & -0,5753 & 0,3733 & 0,0525
\end{array}\right]
$$

Analogamente, segundo a equação 68, a matriz cujas colunas são formadas pelos autovetores normalizados de $A^{*} A$ é: 


$$
V_{v s}=\left[\begin{array}{cccc}
-0,2939 & -0,5797 & 0,7586 & 0,0459 \\
0,8961 & -0,4432 & 0,0098 & -0,0214 \\
0,0201 & -0,0089 & -0,0594 & 0,9980 \\
-0,3319 & -0,6837 & -0,6488 & -0,0380
\end{array}\right]
$$

Realizando a multiplicação de $U_{v s} \Sigma_{v s} V_{v s}^{*}$, encontra-se como resultado, a matriz $A$.

\section{C.2 EXEMPLO NUMÉRICO: ESPAÇO ORTOGONAL DE SÉRIES DE POTÊNCIA}

Neste item será apresentado um exemplo da projeção em uma base ortogonal de séries de potência, apresentada no item 5.3.1.

Aplicando a técnica de projeção em um espaço de séries de potência, também conhecida como Método de Arnoldi, na matriz abaixo, encontra-se:

$$
A=\left[\begin{array}{ccc}
10 & 2 & 3 \\
3 & 4 & 5 \\
0 & 2 & 8
\end{array}\right]
$$

Escolhendo arbitrariamente um vetor $v_{1}$ de módulo unitário:

$$
v_{1}=\left\{\begin{array}{c}
0 \\
0.9492 \\
-0.3147
\end{array}\right\}
$$

temos

$$
\begin{gathered}
\omega_{2}=A v_{1}-\left(\left(A v_{1}\right) \cdot v_{1}\right) v_{1} \\
v_{2}=\frac{\omega_{2}}{\left\|\omega_{2}\right\|}=\left\{\begin{array}{c}
0.9932 \\
0.0367 \\
0.1108
\end{array}\right\} \\
\omega_{3}=A v_{2}-\left(\left(A v_{2}\right) \cdot v_{1}\right) v_{1}-\left(\left(A v_{2}\right) \cdot v_{2}\right) v_{2} \\
v_{3}=\frac{\omega_{3}}{\left\|\omega_{3}\right\|}=\left\{\begin{array}{c}
-0.1167 \\
0.3125 \\
0.9427
\end{array}\right\}
\end{gathered}
$$

A matriz $V_{k r}$ de mudança de base é, portanto, 


$$
V_{k r}=\left[\begin{array}{ccc}
0 & 0.9932 & -0.1167 \\
0.9492 & 0.0367 & 0.3125 \\
-0.3147 & 0.1108 & 0.9427
\end{array}\right]
$$

A matriz $A$ na nova base ortonormal pode ser calculada através do produto

$$
V_{k r}^{T} A V_{k r}=\left[\begin{array}{ccc}
2.3053 & 3.1914 & 2.7585 \\
0.9610 & 10.5083 & 3.3813 \\
0 & 0.8484 & 9.1864
\end{array}\right]
$$

Nota-se que após a transformação de base, a matriz $V_{k r}^{T} A V_{k r}$ é do tipo Hessemberg.

\section{C.3 EXEMPLO NUMÉRICO: MÉTODO DE GUYAN E MÉTODO DE GUYAN MODIFI- CADO}

Neste item serão apresentados exemplos da eliminação de GDL em um sistema pelos métodos de Guyan e Guyan Modificado, descritos nos itens 7.1.1 e 7.1.2 respectivamente.

Os métodos descritos anteriormente, Guyan e Guyan Modificado, resultam em soluções idênticas, salvo variações numéricas oriundas dos algoritmos de inversão de matrizes e solução de sistemas.

O autor selecionou um exemplo didático que ilustra um aplicação onde a utilização destes métodos é vantajosa.

$$
\left[\begin{array}{ll|ll}
a & 0 & 1 & 1 \\
1 & b & 1 & 0 \\
\hline 3 & 1 & 0 & 1 \\
7 & 0 & 1 & 1
\end{array}\right] \cdot\left\{\begin{array}{c}
x_{1} \\
x_{2} \\
\hline x_{3} \\
x_{4}
\end{array}\right\}=\left\{\begin{array}{c}
1 \\
0 \\
2 \\
1
\end{array}\right\}
$$

Dado o sistema linear descrito pela equação 267. encontrar a solução estática

$$
x=\left\{x_{1}, x_{2}, x_{3}, x_{4}\right\}^{T}
$$

que minimize sua distância em relação a $\{1,4,5,2\}^{T}$

Independentemente da quantidade de vezes que o sistema linear será resolvido, a matriz $A_{22}$ será invertida somente uma vez, pois não depende dos parâmetros a e b. Desta maneira temos:

$$
A_{22}^{-1}=\left[\begin{array}{cc}
-1 & 1 \\
1 & 0
\end{array}\right]
$$


Substituindo os valores na equação 108 e 109 , temos respectivamente

$$
\left\{\begin{array}{l}
x_{3} \\
x_{4}
\end{array}\right\}=\left[\begin{array}{c}
-1 \\
2
\end{array}\right]+\left[\begin{array}{cc}
4 & -1 \\
3 & 1
\end{array}\right] \cdot\left\{\begin{array}{l}
x_{1} \\
x_{2}
\end{array}\right\}
$$

e

$$
\left[\begin{array}{cc}
a-7 & 0 \\
-3 & b+1
\end{array}\right] \cdot\left\{\begin{array}{l}
x_{1} \\
x_{2}
\end{array}\right\}=\left\{\begin{array}{l}
0 \\
1
\end{array}\right\}
$$

A equação 270 representa o sistema reduzido cuja solução é idêntica à parcela $\left\{x_{1}, x_{2}\right\}$ do modelo completo.

Aplicando o Método de Guyan Modificado ao mesmo exemplo e substituindo os valores nas equações 123 e 124, temos:

$$
\begin{gathered}
\left\{\begin{array}{l}
x_{3} \\
x_{4}
\end{array}\right\}=\left[\begin{array}{c}
-\frac{2}{5} \\
\frac{8}{5}
\end{array}\right]-\left[\begin{array}{cc}
\frac{a+4}{5} & \frac{1}{5}(3 b-2) \\
\frac{a+14}{5} & \frac{1}{5}(3-2 b)
\end{array}\right] \cdot\left\{\begin{array}{l}
x_{1} \\
x_{2}
\end{array}\right\} \\
{\left[\begin{array}{cc}
3(a-6) & -1(b+1) \\
1-a & 2(b+1)
\end{array}\right] \cdot\left\{\begin{array}{c}
x_{1} \\
x_{2}
\end{array}\right\}=\left\{\begin{array}{c}
-1 \\
2
\end{array}\right\}}
\end{gathered}
$$

\section{C.4 EXEMPLO NUMÉRICO: CORREÇÃO DA RESPOSTA ESTÁTICA DE UM SR}

Neste item será apresentado um exemplo de correção da resposta estática de um SR, apresentada no item 8.2

Dado um sistema completo de ordem $n=6$ que cujo vetor de estados em estado estável para uma entrada degrau é

$$
x_{e q}=\left(\begin{array}{llllll}
1,1 & 1,7 & 2,1 & 3,7 & 0,8 & 0,1
\end{array}\right)^{T}
$$

e a matriz $C$ do sistema completo é

$$
C=\left[\begin{array}{cccccc}
0 & 1 & 0,8 & 1 & 0 & 0,1 \\
1 & 0 & 0.5 & 0 & 1 & 0
\end{array}\right]
$$

Após uma redução por truncamento direto com eliminação de dois graus de liberdade, a ordem do sistema reduzido será $n_{r}=4$ e o vetor de estados em estado estável para entrada degrau é

$$
x_{r e q}=\left(\begin{array}{llll}
1,05 & 1,75 & 2,25 & 3,5
\end{array}\right)^{T}
$$

e a matriz $C_{r}$ do sistema reduzido é: 


$$
C_{r}=\left[\begin{array}{cccc}
0 & 1 & 0,8 & 1 \\
1 & 0 & 0.5 & 0
\end{array}\right]
$$

As respostas de estado estável são:

$$
y_{e q}=C x_{e q}=\left\{\begin{array}{l}
7,09 \\
2,95
\end{array}\right\} \quad y_{r e q}=C_{r} x_{r e q}=\left\{\begin{array}{c}
7,05 \\
2,175
\end{array}\right\}
$$

Os valores da diagonal principal da matriz $E$ são calculados da segunte maneira: $f_{e q 1}=$ $\frac{7,09}{7,05}=1,005674$ e $f_{\text {eq } 1}=\frac{2,95}{2,175}=1,356322$. Desta maneira, pode-se escrever:

$$
E=\left[\begin{array}{cc}
1,005674 & 0 \\
0 & 1,356322
\end{array}\right]
$$

A matriz corrigida $C_{r c}$ é calculada utilizando a equação 160 .

$$
C_{r c}=E C_{r}=\left[\begin{array}{cccc}
0 & 1,00567 & 0,80454 & 1,00567 \\
1,35632 & 0 & 0.67816 & 0
\end{array}\right]
$$

Desta maneira, encontra-se o vetor de saída em estado estável do sistema reduzido e corrigido quando submetido a uma entrada degrau:

$$
y_{\text {rceq }}=C x_{e q}=C_{r c} x_{r e q}=\left\{\begin{array}{l}
7,09 \\
2,95
\end{array}\right\}
$$

Considerando que os sistemas em questão são $\mathrm{LCl}$, quando a resposta estática do sistema é corrigida de acordo com o método descrito acima, o erro estático será sempre nulo para qualquer outra entrada degrau. 